\title{
Reverse Turn Foldamers: An Expanded $\beta$-Turn Motif Reinforced by Double Hydrogen Bonds
}

Quan Tang, ${ }^{1, \$}$ Yulong Zhong, ${ }^{2, \$}$ Daniel P. Miller, ${ }^{3}$ Rui Liu, ${ }^{1}$ Eva Zurek, ${ }^{2}$ Zhong-Lin Lu ${ }^{1, *}$ and Bing Gong ${ }^{2 *}$

${ }^{1}$ College of Chemistry, Beijing Normal University, Beijing 100875, China

${ }^{2}$ Department of Chemistry, University at Buffalo, The State University of New York, Buffalo, New York 14260, USA

${ }^{3}$ Department of Chemistry, Hofstra University, Hempstead, New York 11549, USA

\section{Supporting Information}

$\begin{array}{lll}\text { I. Supporting Figures } & \text { S2 }\end{array}$

$\begin{array}{ll}\text { II. Synthesis and Characterization } & \text { S12 }\end{array}$

$\begin{array}{ll}\text { III. Computational Study } & \text { S64 }\end{array}$

$\begin{array}{ll}\text { IV. References } & \text { S65 }\end{array}$ 


\section{Supplementary Figures}
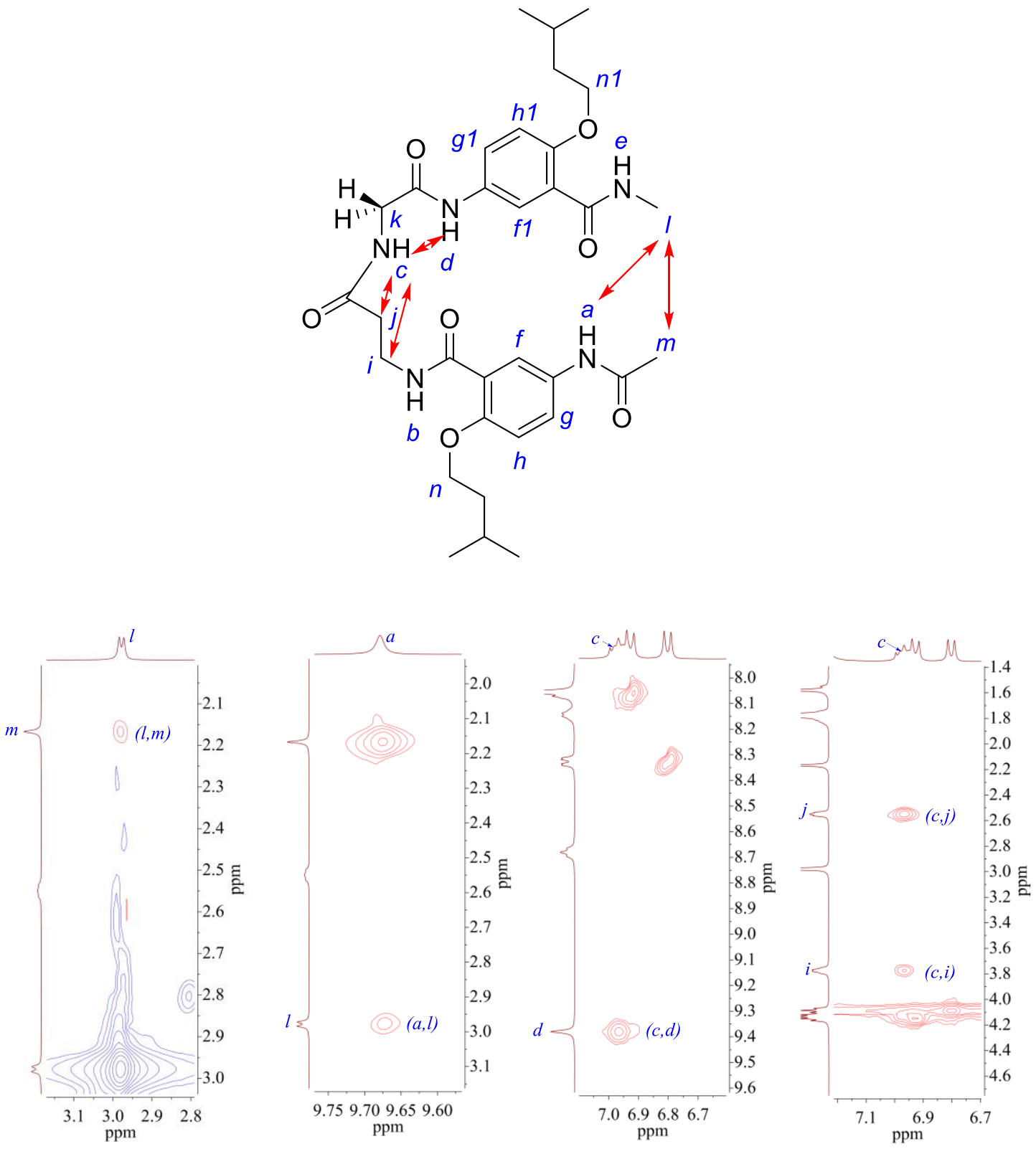

Figure S1a. Labeled structure including observed NOE and partial NOESY spectra of 2a (5 mM, 298 $\mathrm{K}, 400 \mathrm{MHz}$, mixing time: $300 \mathrm{~ms}$ ) in $\mathrm{CDCl}_{3}$. 


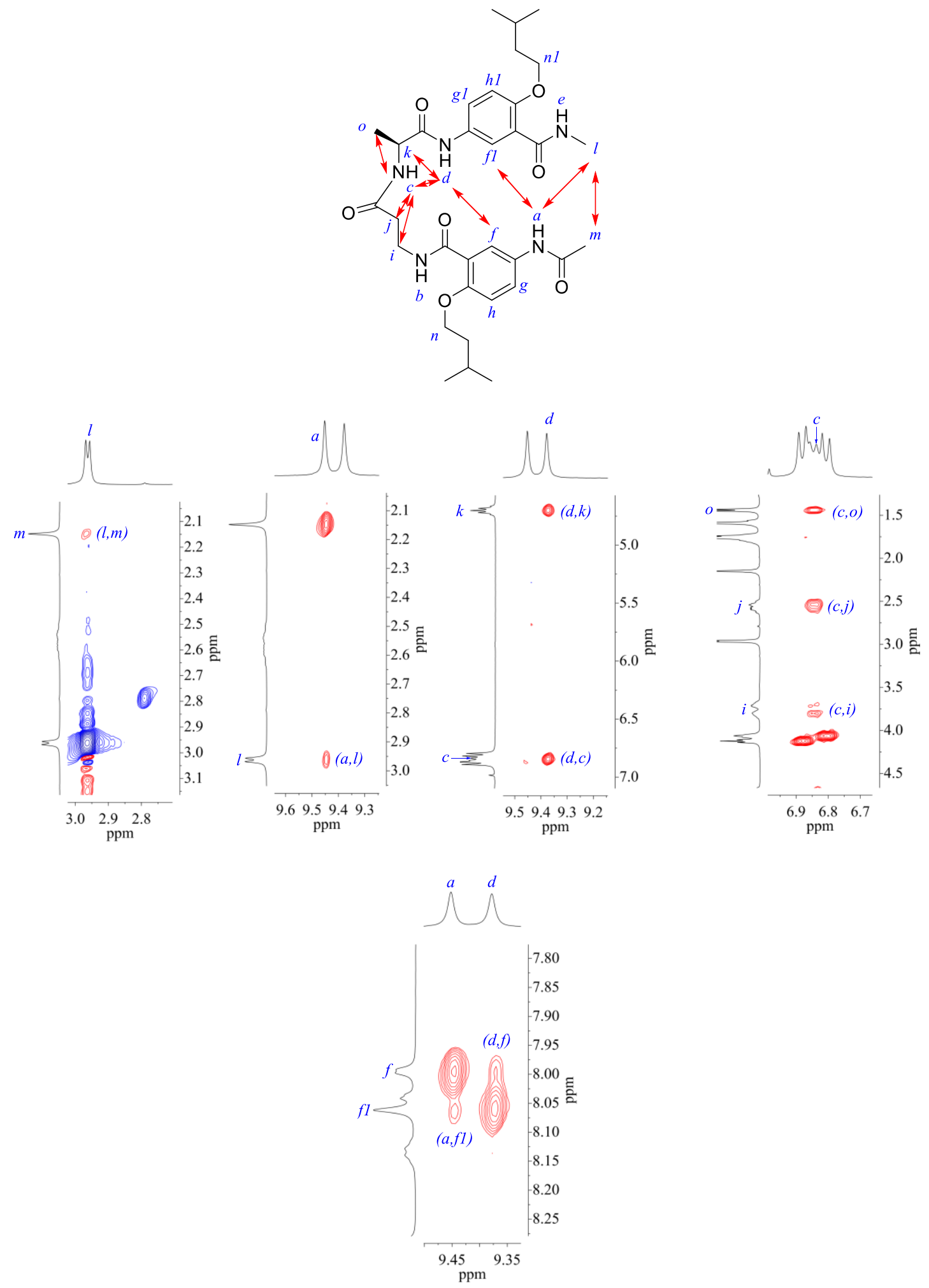

Figure S1b. Labeled structure including observed NOE and partial NOESY spectra of $\mathbf{2 b}(5 \mathrm{mM}, 298$ $\mathrm{K}, 400 \mathrm{MHz}$, mixing time: $300 \mathrm{~ms}$ ) in $\mathrm{CDCl}_{3}$. 

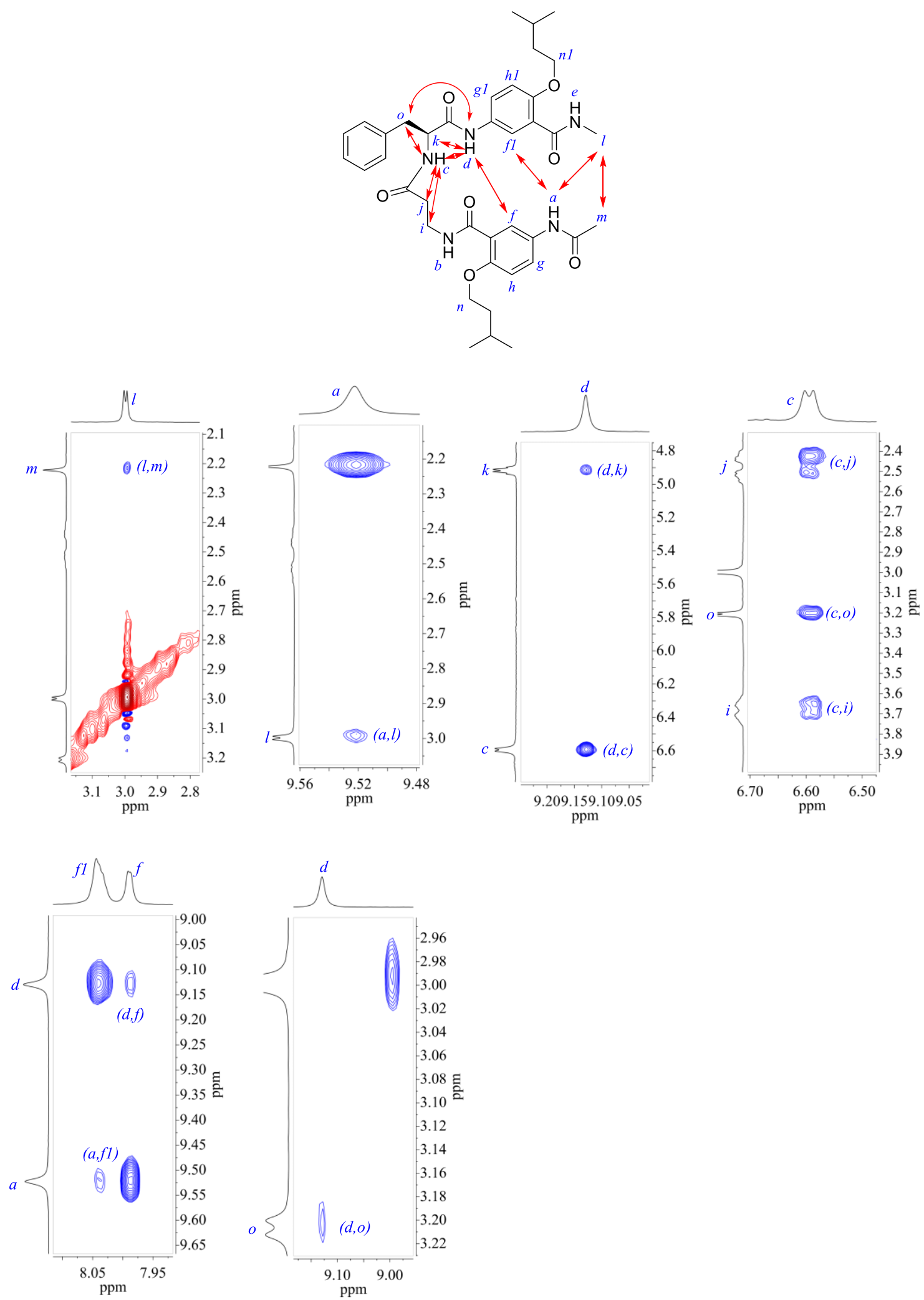

Figure S1c. Labeled structure including observed NOE and partial NOESY spectra of $2 \mathbf{c}(5 \mathrm{mM}, 298$ $\mathrm{K}, 400 \mathrm{MHz}$, mixing time: $300 \mathrm{~ms}$ ) in $\mathrm{CDCl}_{3}$. 


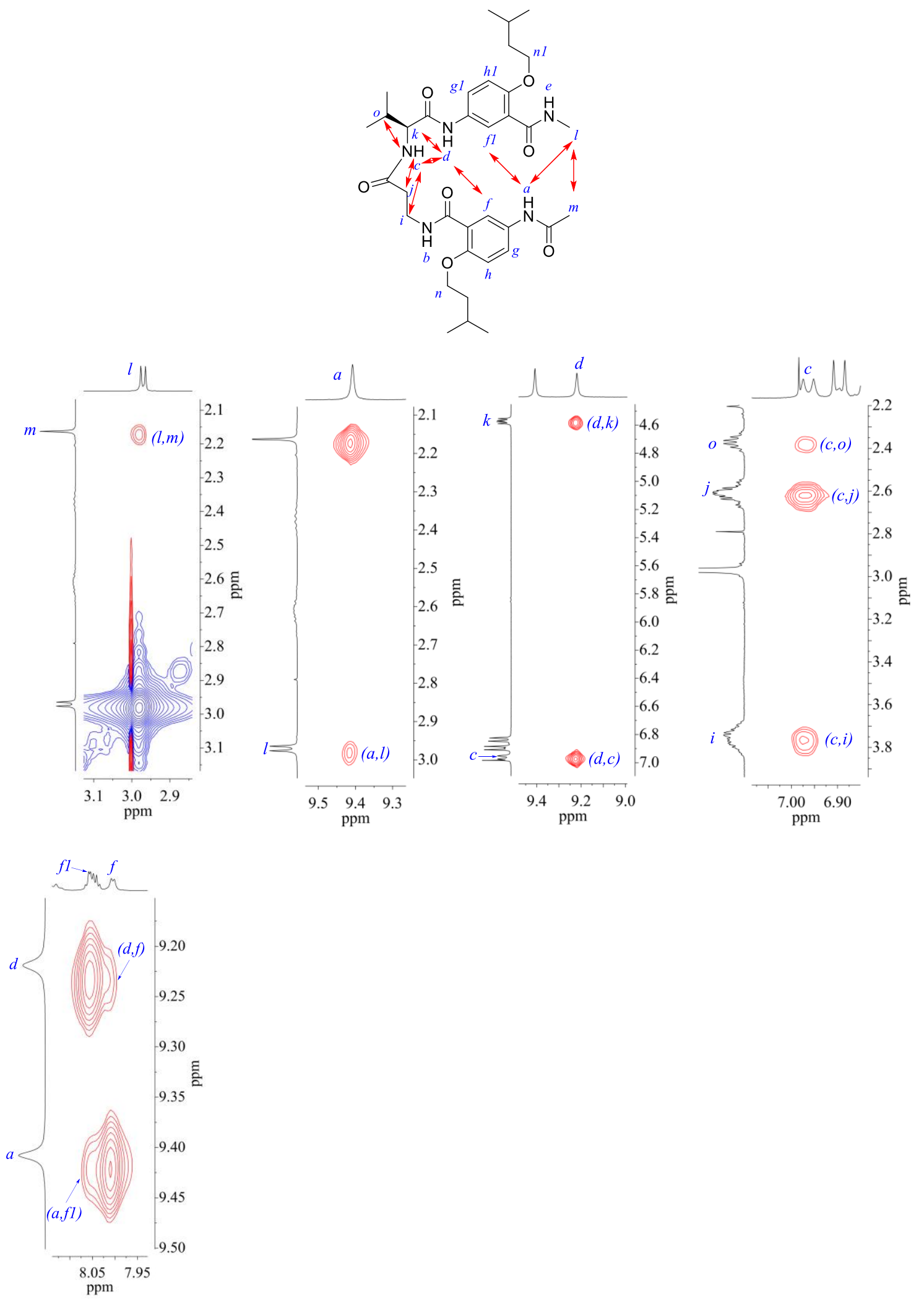

Figure S1d. Labeled structure including observed NOE and partial NOESY spectra of 2 d (5 mM, 298 $\mathrm{K}, 400 \mathrm{MHz}$, mixing time: $300 \mathrm{~ms}$ ) in $\mathrm{CDCl}_{3}$. 


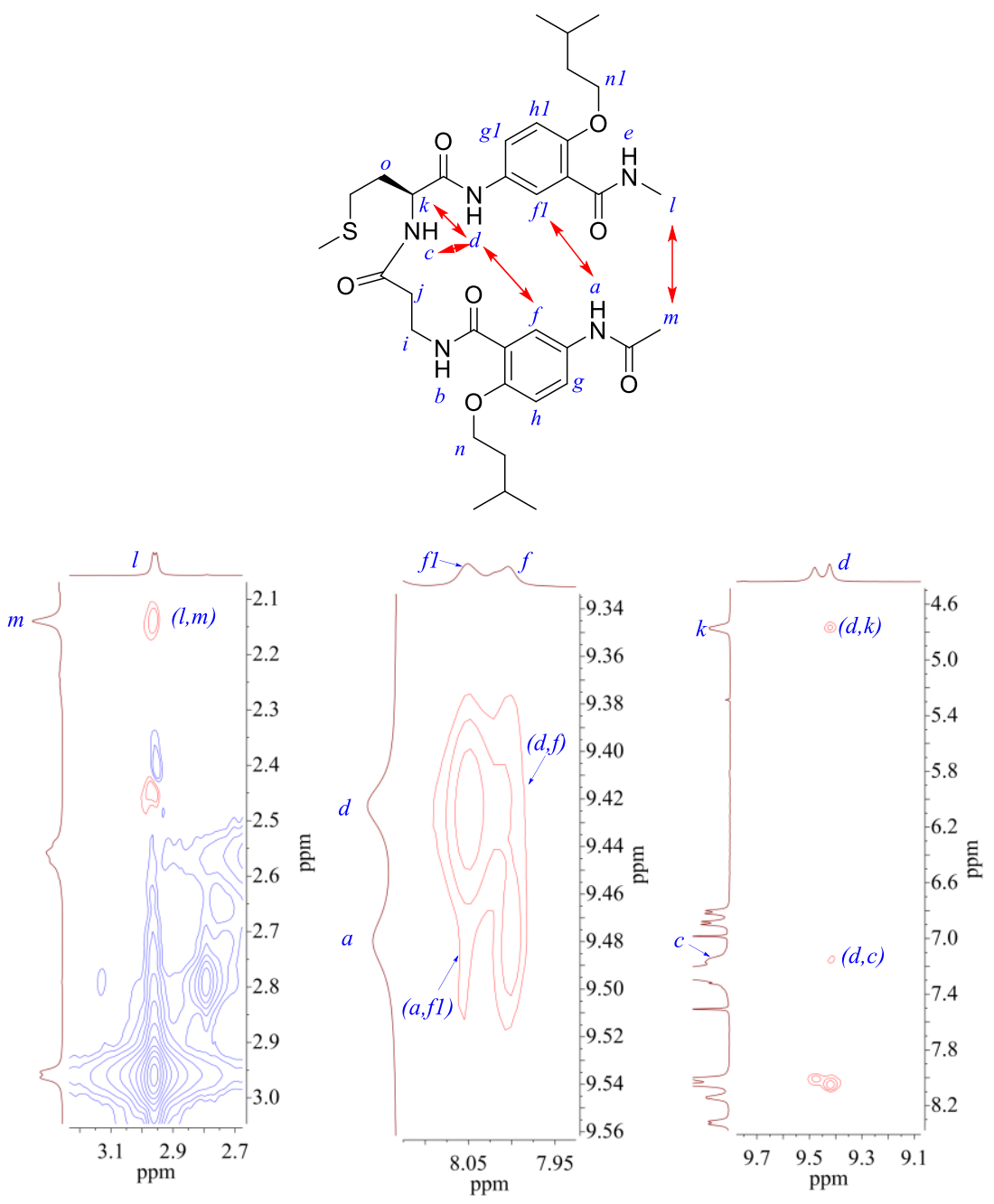

Figure S1e. Labeled structure including observed NOE and partial NOESY spectra of 2 e $(5 \mathrm{mM}, 298$ $\mathrm{K}, 400 \mathrm{MHz}$, mixing time: $300 \mathrm{~ms}$ ) in $\mathrm{CDCl}_{3}$. 

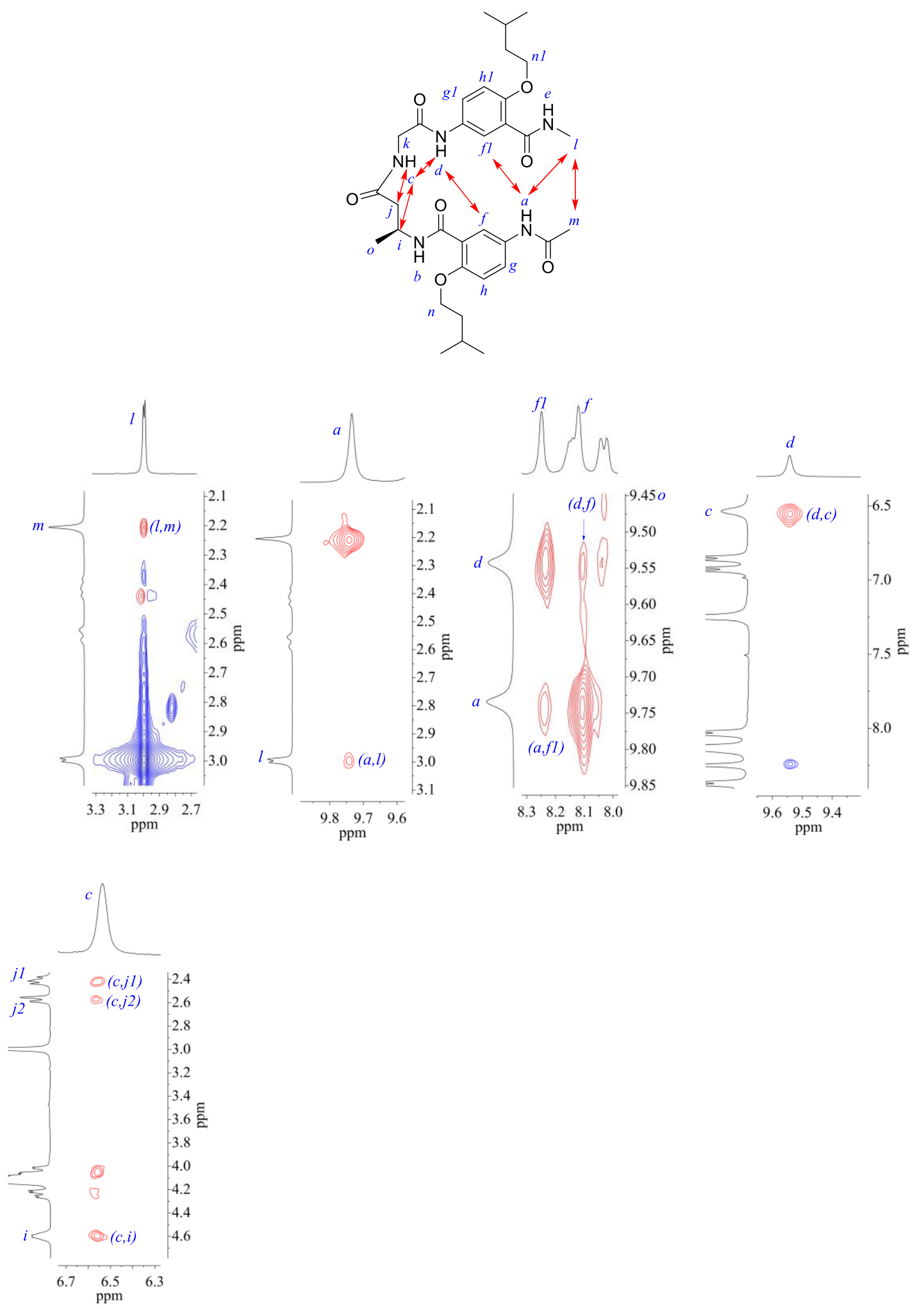

Figure S1f. Labeled structure including observed NOE and partial NOESY spectra of $\mathbf{2 f}(5 \mathrm{mM}, 298$ $\mathrm{K}, 400 \mathrm{MHz}$, mixing time: $300 \mathrm{~ms}$ ) in $\mathrm{CDCl}_{3}$. 

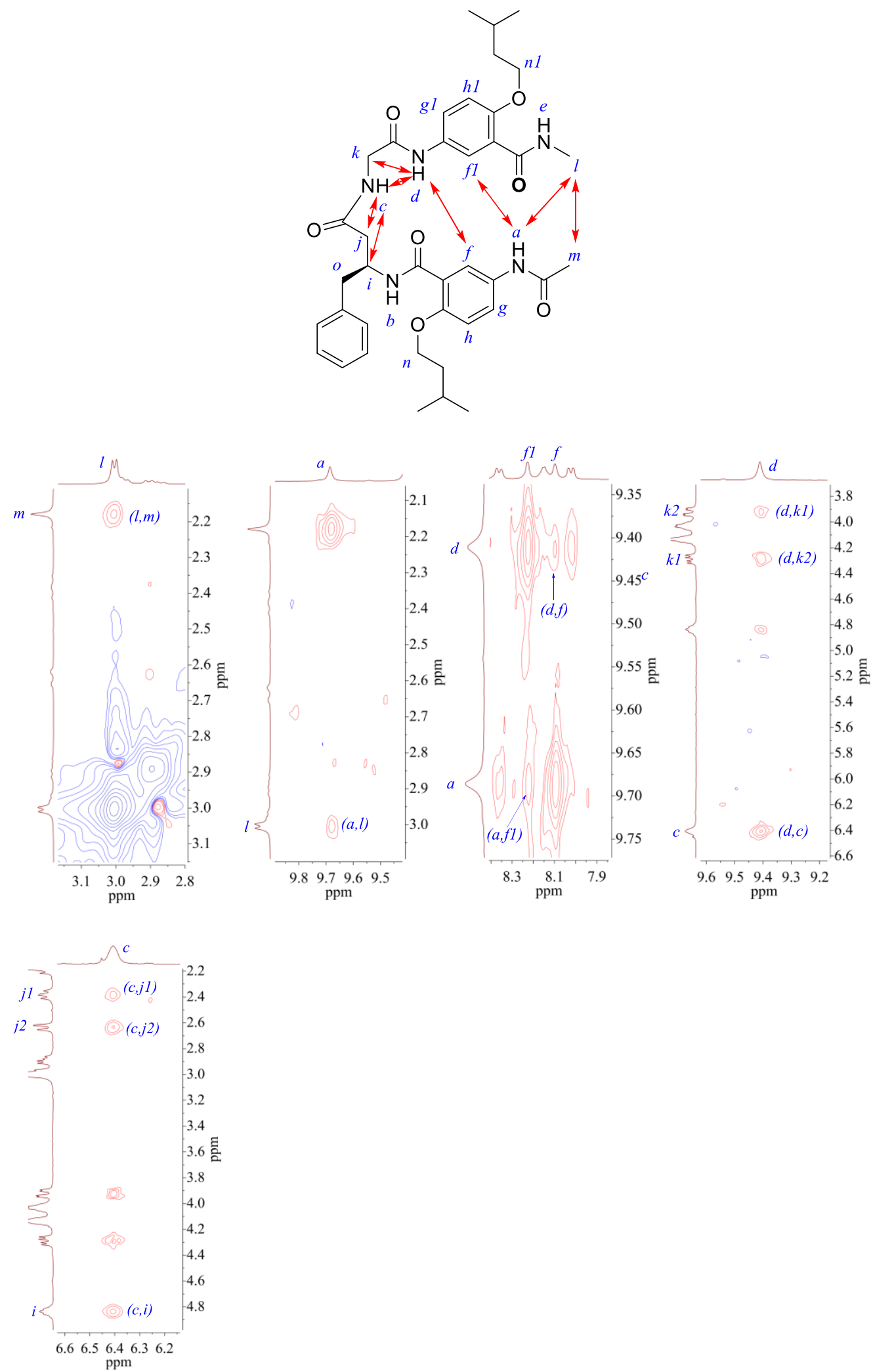

Figure S1g. Labeled structure including observed NOE and partial NOESY spectrum of $\mathbf{2 g}(5 \mathrm{mM}, 298$ $\mathrm{K}, 400 \mathrm{MHz}$, mixing time: $300 \mathrm{~ms}$ ) in $\mathrm{CDCl}_{3}$. 


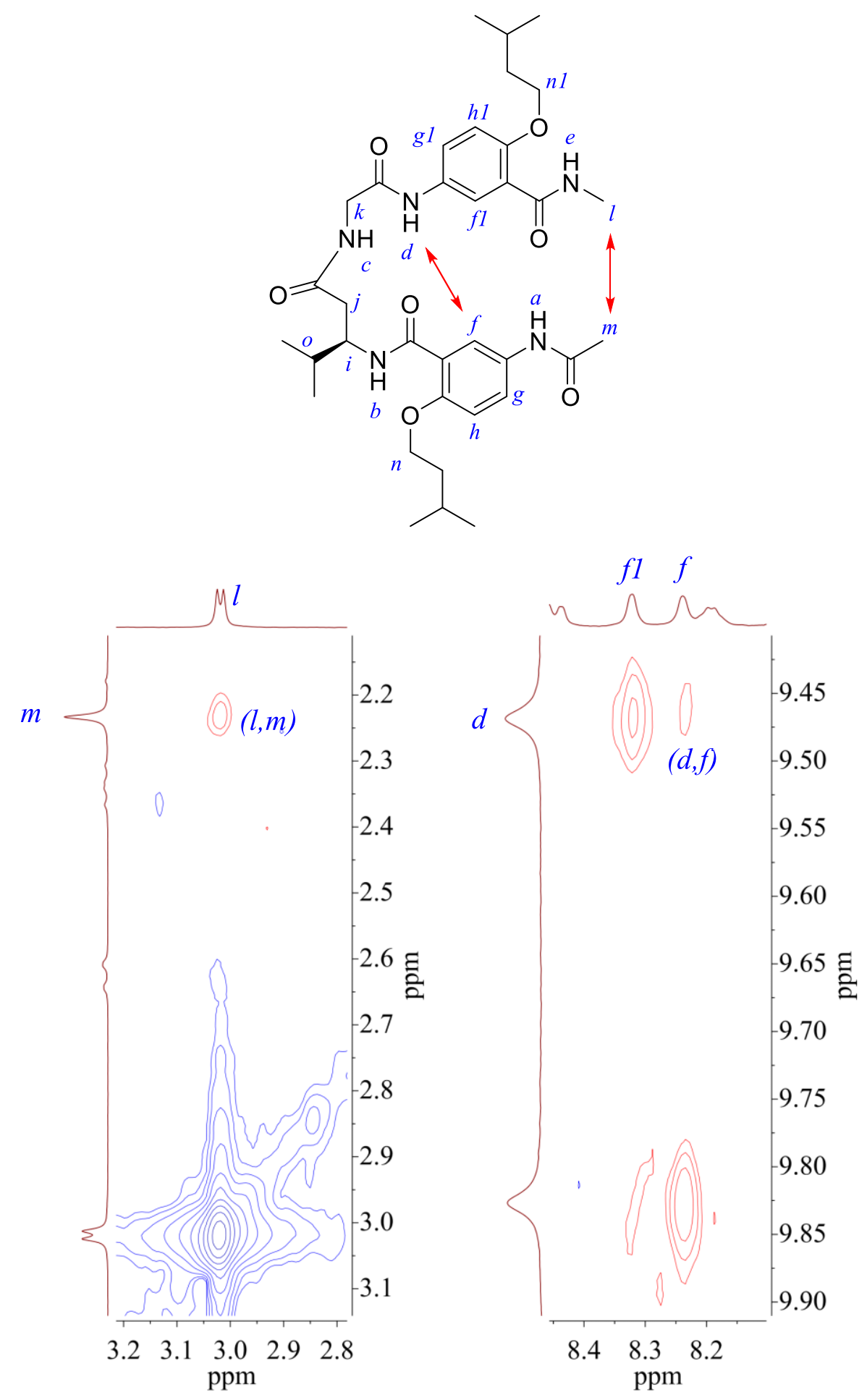

Figure S1h. Labeled structure including observed NOE and partial NOESY spectra of $\mathbf{2 h}(1 \mathrm{mM}, 298$ $\mathrm{K}, 400 \mathrm{MHz}$, mixing time: $300 \mathrm{~ms}$ ) in $\mathrm{CDCl}_{3}$. 

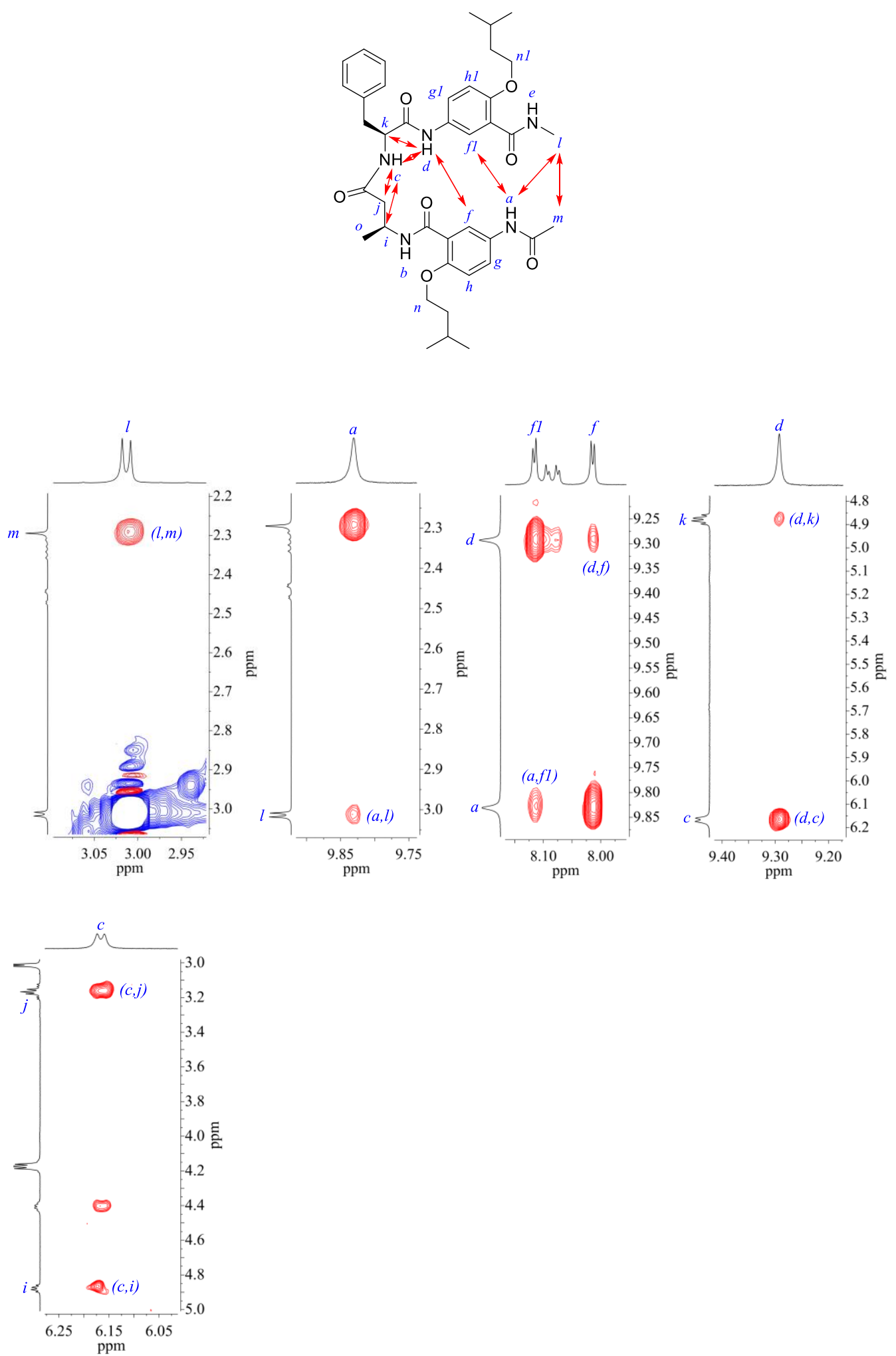

Figure S1i. Labeled structure including observed NOE and partial NOESY spectra of $\mathbf{2 i}$ ( $5 \mathrm{mM}, 298 \mathrm{~K}$, $500 \mathrm{MHz}$, mixing time: $300 \mathrm{~ms}$ ) in $\mathrm{CDCl}_{3}$. 


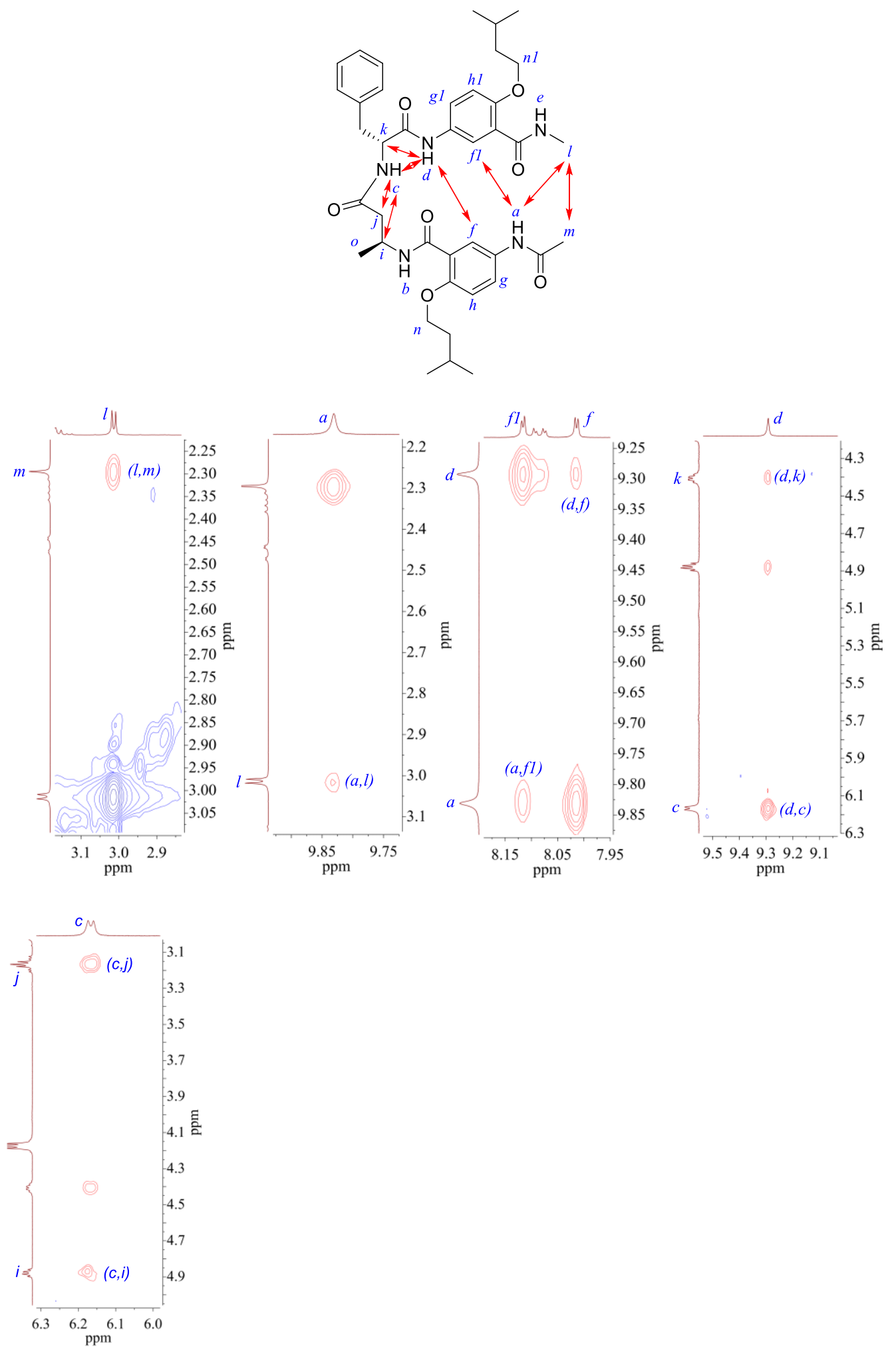

Figure S1j. Labeled structure including observed NOE and partial NOESY spectra of $\mathbf{2 j}$ (5 mM, $298 \mathrm{~K}$, $500 \mathrm{MHz}$, mixing time: $300 \mathrm{~ms}$ ) in $\mathrm{CDCl}_{3}$. 


\section{Synthesis and Characterization}

\section{General remarks}

Reagents and solvents were purchased from commercial sources and used without further purification. Column chromatography was carried out on silica gel (300 400 mesh). ${ }^{1} \mathrm{H}$ NMR spectra were recorded at $400 \mathrm{MHz}$ and $600 \mathrm{MHz}$ on a Bruker-400 spectrometer and JEOL-400 and 600 spectrometers at ambient temperature. ${ }^{13} \mathrm{C}$ NMR spectra were measured at $100 \mathrm{MHz}$ and $150 \mathrm{MHz}$ on the same spectrometers. Chemical shifts are reported in parts per million downfield from TMS (tetramethylsilane). Coupling constant in ${ }^{1} \mathrm{H}$ NMR are expressed in Hertz. Electroscpray ionization high resolution mass spectra (ESI-HRMS) were recorded on a High resolution mass spectra using a waters LCT Premier XE spectrometer (Waters, Milford, MA, USA)

\section{Scheme S1. Synthesis of peptides $2 \mathrm{a}-\mathbf{2 j}$}

(a) Preparation of dipeptide

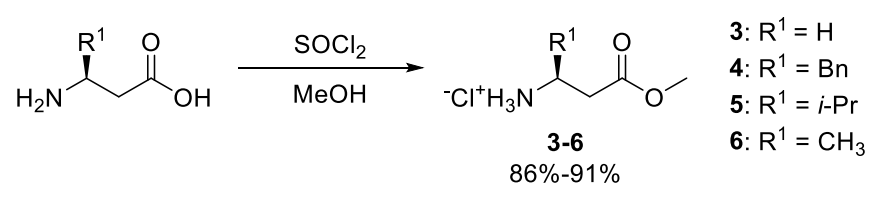

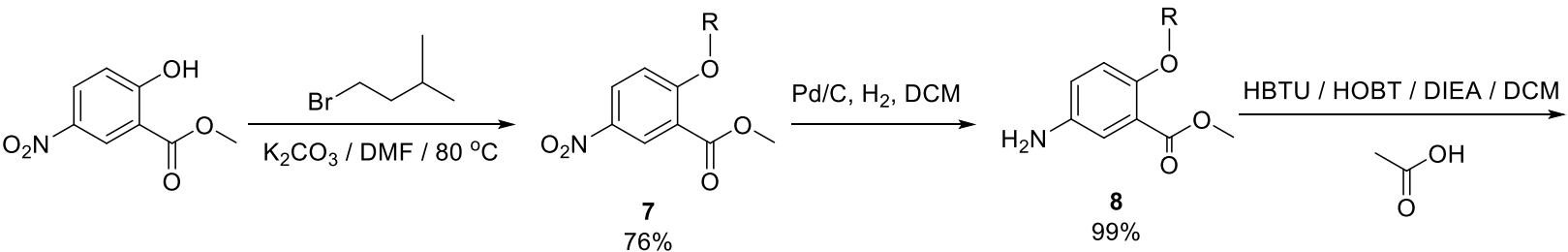
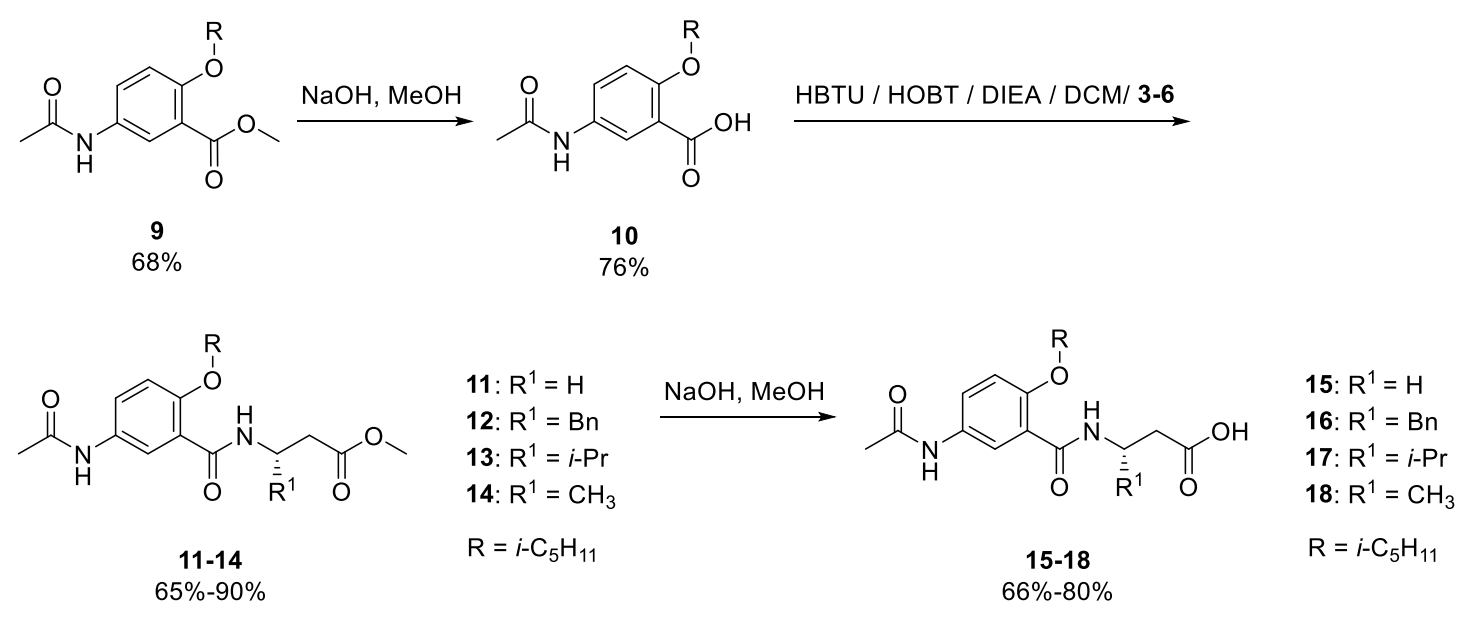

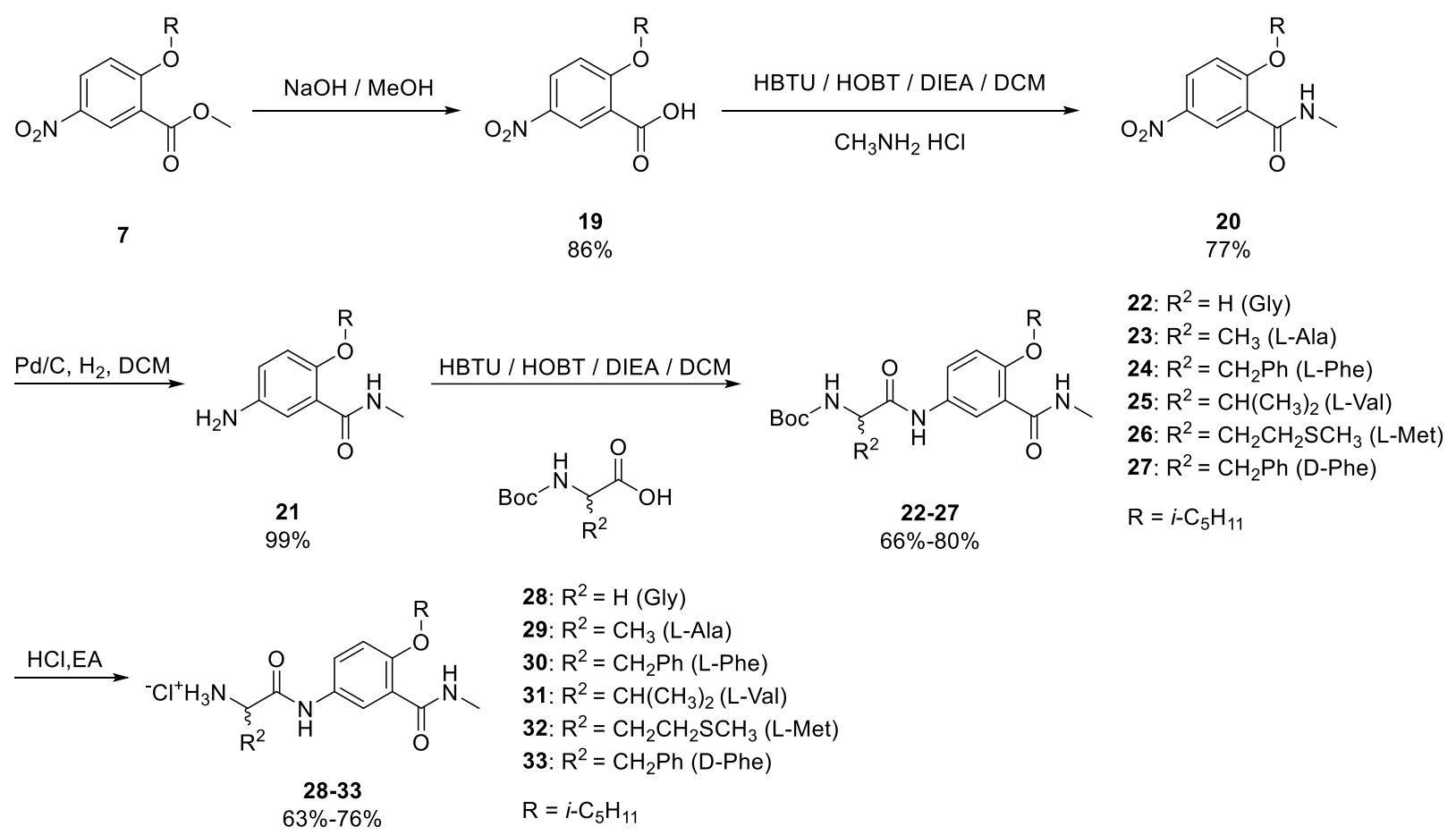

(b) Preparation of peptides $\mathbf{2} \mathbf{a}-\mathbf{2} \mathbf{j}$
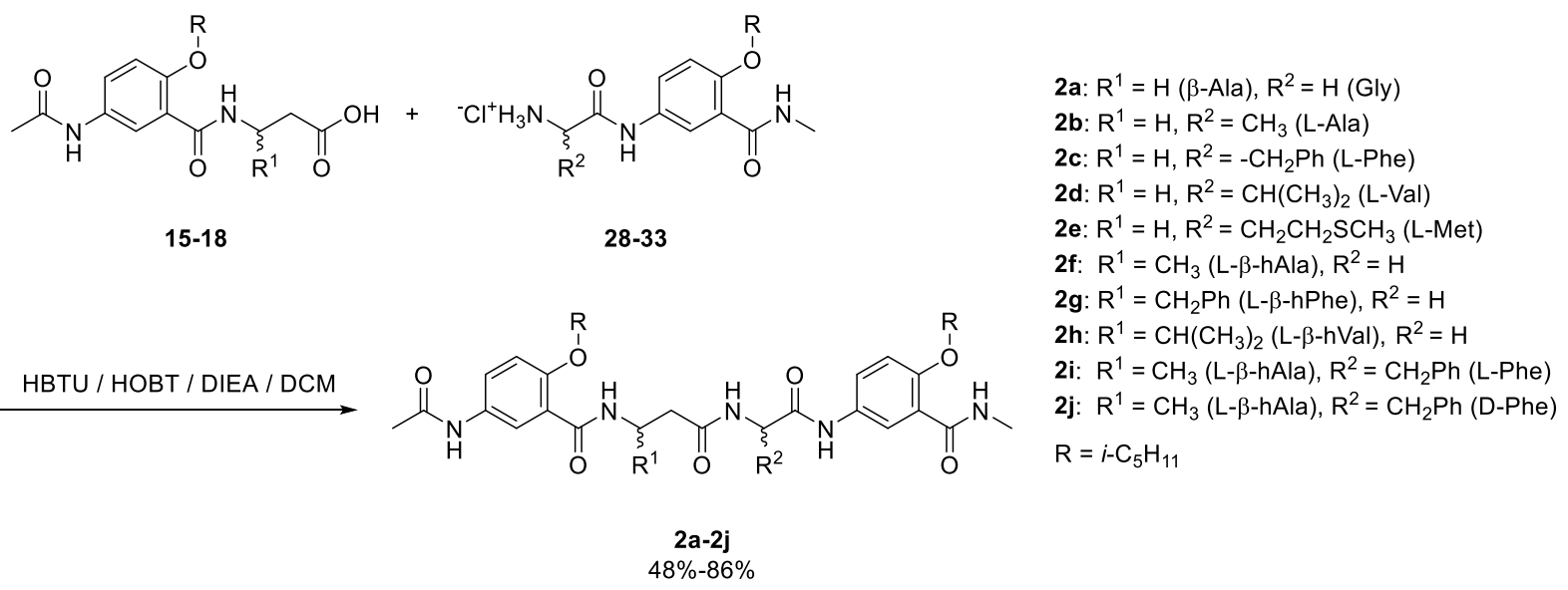

Compound $3,4,5$ and 6 were synthesized according to previously reported procedures. ${ }^{1-2}$

Compound 7. A mixture of methyl 2-hydroxy-5-nitrobenzoate ( $25.00 \mathrm{~g}, 126.81 \mathrm{mmol})$, $\mathrm{K}_{2} \mathrm{CO}_{3}(70.10 \mathrm{~g}, 507.24 \mathrm{mmol})$, 1-bromo-3-methylbutane (22.99 g, $\left.152.17 \mathrm{mmol}\right)$, KI $(1.25 \mathrm{~g}, 7.53 \mathrm{mmol})$ and DMF $(250 \mathrm{~mL})$ was heated in oil bath at $80^{\circ} \mathrm{C}$ for $10 \mathrm{~h}$. The $\mathrm{K}_{2} \mathrm{CO}_{3}$ was filtrated and washed by ethyl acetate. The filtrate and the extract were combined. After removing the solvent under reduced pressure, the remaining residue was dissolved in methylene chloride, washed with diluted $\mathrm{HCl}$ and brine, and dried over anhydrous $\mathrm{Na}_{2} \mathrm{SO}_{4}$. Removal of methylene chloride afforded the desired product as a yellow solid (25.74 g, 76\%). ${ }^{1} \mathrm{H}$ NMR (400 MHz, DMSO- $\left.d_{6}\right) \delta 8.43(\mathrm{~d}, J=3.0 \mathrm{~Hz}, 1 \mathrm{H})$, 
8.35 (dd, $J=9.2,3.0 \mathrm{~Hz}, 1 \mathrm{H}), 7.37$ (d, $J=9.2 \mathrm{~Hz}, 1 \mathrm{H}), 4.22(\mathrm{t}, J=6.5 \mathrm{~Hz}, 2 \mathrm{H}), 3.33$ (s, $3 \mathrm{H}), 1.90-1.76(\mathrm{~m}, 1 \mathrm{H}), 1.69-1.60(\mathrm{~m}, 2 \mathrm{H}), 0.93(\mathrm{~d}, J=6.6 \mathrm{~Hz}, 6 \mathrm{H}) .{ }^{13} \mathrm{C}$ NMR $(101$ $\left.\mathrm{MHz}, \mathrm{CDCl}_{3}\right) \delta 164.8,163.3,140.5,128.9,128.0,120.8,112.8,68.5,52.6,37.6,25.1,22.6$. ESI-MS: calcd for $\mathrm{C}_{13} \mathrm{H}_{17} \mathrm{NO}_{5}\left(\mathrm{M}+\mathrm{Na}^{+}\right) 290.10$ found 290.13 .

Compound 8. Compound 7 ( $20.00 \mathrm{~g}, 74.87 \mathrm{mmol})$ was reduced by catalytic hydrogenation in dichloromethane $(50 \mathrm{~mL})$ at room temperature and atmospheric pressure, using $\mathrm{Pd}-\mathrm{C}$ $(0.20 \mathrm{~g}, 10 \%)$ as the catalyst. After all substrate was reduced, removal of catalyst and solvent gave the product as yellow oil. This compound was used without further purification.

Compound 9. To a solution of acetic acid $(5.40 \mathrm{~g}, 88.92 \mathrm{mmol})$ in $20 \mathrm{~mL}$ dichloromethane, HBTU (33.72 g, $88.92 \mathrm{mmol})$, HOBt (12.01 g, $88.92 \mathrm{mmol}$ ) and $N$, $N$-diisopropylethylamin $(22.99 \mathrm{~g}, 177.84 \mathrm{mmol})$ was added at $0{ }^{\circ} \mathrm{C} .30 \mathrm{~min}$ later, the Compound 8 (17.56 $\mathrm{g}, 74.09 \mathrm{mmol}$ ) in $15 \mathrm{~mL}$ dichloromethane was added to the mixture. Then the reaction was allowed to proceed for $8 \mathrm{~h}$ at room temperature and inert atmosphere. The mixture was washed with diluted $\mathrm{HCl}$ and saturated brine, and solvent was removed in vacuum. Purification was accomplished by chromatography on silica gel using dichloromethane/MeOH to afford 9 as a white solid (14.06 g, 68\%). ${ }^{1} \mathrm{H}$ NMR $(600 \mathrm{MHz}$, $\left.\mathrm{CDCl}_{3}\right) \delta 7.77(\mathrm{dd}, J=9.0,2.8 \mathrm{~Hz}, 1 \mathrm{H}), 7.71(\mathrm{~d}, J=2.8 \mathrm{~Hz}, 1 \mathrm{H}), 7.24(\mathrm{~s}, 1 \mathrm{H}), 6.93(\mathrm{~d}, J=$ $9.0 \mathrm{~Hz}, 1 \mathrm{H}), 4.03(\mathrm{t}, J=6.7 \mathrm{~Hz}, 2 \mathrm{H}), 3.86(\mathrm{~s}, 3 \mathrm{H}) 2.16(\mathrm{~s}, 3 \mathrm{H}), 1.91-1.81(\mathrm{~m}, 1 \mathrm{H}), 1.75$ $-1.65(\mathrm{~m}, 2 \mathrm{H}), 0.95(\mathrm{~d}, J=6.7 \mathrm{~Hz}, 6 \mathrm{H}) .{ }^{13} \mathrm{C}$ NMR $\left(151 \mathrm{MHz}, \mathrm{CDCl}_{3}\right) \delta 168.4,166.6$, 155.7, 130.6, 126.0, 123.6, 120.5, 114.1, 68.0, 52.2, 38.0, 25.2, 24.5, 22.7. HRMS(ESI): calcd for $\mathrm{C}_{15} \mathrm{H}_{21} \mathrm{NO}_{4}: 280.1543\left(\mathrm{M}+\mathrm{H}^{+}\right)$, found:280.1540 $\left(\mathrm{M}+\mathrm{H}^{+}\right)$.

Compound 10. The ester $9(10.00 \mathrm{~g}, 35.82 \mathrm{mmol})$ was dissolved in $\mathrm{MeOH}(100 \mathrm{~mL})$, to which $2 \mathrm{~N} \mathrm{NaOH}(71.60 \mathrm{~mL}, 143.20 \mathrm{mmol})$ was added. The mixture was heated under reflux in oil bath for $2 \mathrm{~h}$. After removed the $\mathrm{MeOH}$ under vacuum, the aqueous layer was acidulated by addition of $3 \mathrm{~N} \mathrm{HCl}$ to $\mathrm{pH} 2$ followed by filtration to obtain a white solid (7.21 g, 76\%). ${ }^{1} \mathrm{H}$ NMR (600 MHz, $\left.\mathrm{CDCl}_{3}\right) 11.27$ (s, 1H), 8.94 (s, $\left.1 \mathrm{H}\right), 8.39$ (dd, $J=9.0$, $2.8 \mathrm{~Hz}, 1 \mathrm{H}), 8.04(\mathrm{~d}, J=2.8 \mathrm{~Hz}, 1 \mathrm{H}), 7.01(\mathrm{~d}, J=9.1 \mathrm{~Hz}, 1 \mathrm{H}), 4.25$ (t, $J=6.4 \mathrm{~Hz}, 2 \mathrm{H})$, $2.23(\mathrm{~s}, 3 \mathrm{H}), 1.86-1.74(\mathrm{~m}, 3 \mathrm{H}), 0.98(\mathrm{~d}, J=6.2 \mathrm{~Hz}, 6 \mathrm{H}) .{ }^{13} \mathrm{C} \mathrm{NMR}\left(151 \mathrm{MHz}, \mathrm{CDCl}_{3}\right) \delta$ 169.5, 166.1, 153.7, 133.7, 127.3, 124.2, 117.3, 113.4, 69.2, 37.7, 25.2, 24.4, 22.5. HRMS(ESI): calcd for $\mathrm{C}_{14} \mathrm{H}_{19} \mathrm{NO}_{4}: 264.1241\left(\mathrm{M}-\mathrm{H}^{+}\right)$, found: $264.1239\left(\mathrm{M}-\mathrm{H}^{+}\right)$.

Compound 11. To a solution of compound $10(2.00 \mathrm{~g}, 7.54 \mathrm{mmol})$ in $20 \mathrm{~mL}$ dichloromethane, HBTU (3.43 g, $9.05 \mathrm{mmol})$, $\mathrm{HOBt}(1.22 \mathrm{~g}, 9.05 \mathrm{mmol})$ and $\mathrm{N}, \mathrm{N}$ diisopropyl-ethylamin $(2.43 \mathrm{~g}, 18.85 \mathrm{mmol})$ was added at $0{ }^{\circ} \mathrm{C} .30$ min later, the compound $3(1.05 \mathrm{~g}, 7.54 \mathrm{mmol})$ in $15 \mathrm{~mL}$ dichloromethane was added to the mixture. Then the reaction was allowed to proceed for $3 \mathrm{~h}$ at room temperature and inert atmosphere. The mixture was washed with diluted $\mathrm{HCl}$ and saturated brine, and solvent was removed in 
vacuum. The crude product was purified by column chromatography (petroleum ether/acetone $=5 / 1)$ to afford a white solid $(2.38 \mathrm{~g}, 90 \%) .{ }^{1} \mathrm{H}$ NMR $\left(400 \mathrm{MHz}\right.$, DMSO- $\left.d_{6}\right)$ $\delta 9.91(\mathrm{~s}, 1 \mathrm{H}), 8.29$ (t, $J=5.8 \mathrm{~Hz}, 1 \mathrm{H}), 7.95(\mathrm{~d}, J=2.8 \mathrm{~Hz}, 1 \mathrm{H}), 7.75(\mathrm{dd}, J=8.9,2.8 \mathrm{~Hz}$, $1 \mathrm{H}), 7.09(\mathrm{~d}, J=9.0 \mathrm{~Hz}, 1 \mathrm{H}), 4.08(\mathrm{t}, J=6.6 \mathrm{~Hz}, 2 \mathrm{H}), 3.62(\mathrm{~s}, 3 \mathrm{H}), 3.52(\mathrm{q}, J=6.4 \mathrm{~Hz}$, $2 \mathrm{H}), 2.58(\mathrm{t}, J=6.5 \mathrm{~Hz}, 2 \mathrm{H}), 2.01(\mathrm{~s}, 3 \mathrm{H}), 1.83-1.62(\mathrm{~m}, 3 \mathrm{H}), 0.94(\mathrm{~d}, J=6.5 \mathrm{~Hz}, 6 \mathrm{H})$. ${ }^{13} \mathrm{C}$ NMR $\left(151 \mathrm{MHz}\right.$, DMSO- $\left.d_{6}\right) \delta 172.1,167.9,164.4,152.2,132.6,123.1,122.1,121.5$, 113.4, 67.4, 51.4, 37.1, 35.0, 33.6, 24.7, 23.8, 22.4. ESI-MS: calcd for $\mathrm{C}_{18} \mathrm{H}_{26} \mathrm{~N}_{2} \mathrm{O}_{5}\left(\mathrm{M}+\mathrm{Na}^{+}\right)$ 373.17 found 373.27 .

Compound 12. To a solution of compound $10(2.00 \mathrm{~g}, 7.54 \mathrm{mmol})$ in $20 \mathrm{~mL}$ dichloromethane, HBTU (3.43 g, $9.05 \mathrm{mmol})$, HOBt (1.22 g, $9.05 \mathrm{mmol})$ and $\mathrm{N}, \mathrm{N}$ diisopropyl-ethylamin $(2.43 \mathrm{~g}, 18.85 \mathrm{mmol})$ was added at $0{ }^{\circ} \mathrm{C} .30$ min later, the compound $4(1.73 \mathrm{~g}, 7.54 \mathrm{mmol})$ in $15 \mathrm{~mL}$ dichloromethane was added to the mixture. Then the reaction was allowed to proceed for $3 \mathrm{~h}$ at room temperature and inert atmosphere. The mixture was washed with diluted $\mathrm{HCl}$ and saturated brine. The combined organic layers were dried with sodium sulfate. The crude product was purified by column chromatography (petroleum ether/acetone $=5 / 1)$ to afford a white solid $(2.16 \mathrm{~g}, 65 \%) .{ }^{1} \mathrm{H}$ NMR $(600 \mathrm{MHz}$, DMSO- $\left.d_{6}\right) \delta 9.90(\mathrm{~s}, 1 \mathrm{H}), 8.28(\mathrm{t}, J=5.8 \mathrm{~Hz}, 1 \mathrm{H}), 7.93(\mathrm{~d}, J=2.8 \mathrm{~Hz}, 1 \mathrm{H}), 7.71(\mathrm{dd}, J=$ 8.9, $2.8 \mathrm{~Hz}, 1 \mathrm{H}), 7.34-7.27(\mathrm{~m}, 2 \mathrm{H}), 7.25-7.19(\mathrm{~m}, 3 \mathrm{H}), 7.06(\mathrm{~d}, J=9.0 \mathrm{~Hz}, 1 \mathrm{H}), 4.05$ $(\mathrm{t}, J=6.6 \mathrm{~Hz}, 2 \mathrm{H}), 3.70-3.62(\mathrm{~m}, 1 \mathrm{H}), 3.52(\mathrm{~s}, 3 \mathrm{H}), 3.00(\mathrm{dd}, J=13.6,5.7 \mathrm{~Hz}, 1 \mathrm{H}), 2.77$ (dd, $J=13.7,8.4 \mathrm{~Hz}, 1 \mathrm{H}), 2.60$ (dd, $J=16.8,7.1 \mathrm{~Hz}, 1 \mathrm{H}), 2.52(\mathrm{dd}, J=16.8,5.5 \mathrm{~Hz}, 1 \mathrm{H}$ ), $1.97(\mathrm{~s}, 3 \mathrm{H}), 1.78-1.61(\mathrm{~m}, 3 \mathrm{H}), 0.90(\mathrm{~d}, J=6.6 \mathrm{~Hz}, 6 \mathrm{H}) .{ }^{13} \mathrm{C}$ NMR $(151 \mathrm{MHz}$, DMSO$\left.d_{6}\right) \delta 173.1,170.2,168.0,164.3,152.2,135.8,132.6,129.4,128.7,127.1,123.2,122.0$, 121.6, 113.4, 67.5, 51.8, 48.8, 38.0, 37.1, 35.8, 35.0, 33.8, 24.7, 23.8, 22.4. ESI-MS: calcd for $\mathrm{C}_{25} \mathrm{H}_{32} \mathrm{~N}_{2} \mathrm{O}_{5}\left(\mathrm{M}+\mathrm{H}^{+}\right) 441.24$ found 441.29 .

Compound 13. To a solution of compound $10(2.00 \mathrm{~g}, 7.54 \mathrm{mmol})$ in $20 \mathrm{~mL}$ dichloromethane, HBTU (3.43 g, $9.05 \mathrm{mmol})$, HOBt $(1.22 \mathrm{~g}, 9.05 \mathrm{mmol})$ and $\mathrm{N}, \mathrm{N}$ diisopropyl-ethylamin $(2.43 \mathrm{~g}, 18.85 \mathrm{mmol})$ was added at $0{ }^{\circ} \mathrm{C} .30$ min later, the compound $5(1.37 \mathrm{~g}, 7.54 \mathrm{mmol})$ in $15 \mathrm{~mL}$ dichloromethane was added to the mixture. Then the reaction was allowed to proceed for $3 \mathrm{~h}$ at room temperature and inert atmosphere. The mixture was washed with diluted $\mathrm{HCl}$ and saturated brine, and solvent was removed in vacuum. The crude product was purified by column chromatography (petroleum ether/acetone $=5 / 1)$ to afford a white solid $(2.66 \mathrm{~g}, 90 \%) .{ }^{1} \mathrm{H}$ NMR $(400 \mathrm{MHz}$, Chloroformd) $\delta 8.33(\mathrm{~d}, J=9.1 \mathrm{~Hz}, 1 \mathrm{H}), 8.12(\mathrm{dd}, J=9.1,2.7 \mathrm{~Hz}, 1 \mathrm{H}), 7.89-7.81(\mathrm{~m}, 2 \mathrm{H}), 6.95(\mathrm{~d}$, $J=8.9 \mathrm{~Hz}, 1 \mathrm{H}), 4.41-4.32(\mathrm{~m}, 1 \mathrm{H}), 4.15(\mathrm{t}, J=6.5 \mathrm{~Hz}, 2 \mathrm{H}), 3.65(\mathrm{~s}, 3 \mathrm{H}), 2.66-2.47$ (m, $2 \mathrm{H}), 2.19(\mathrm{~s}, 3 \mathrm{H}), 2.03-1.77(\mathrm{~m}, 4 \mathrm{H}), 1.07-0.95(\mathrm{~m}, 12 \mathrm{H}) .{ }^{13} \mathrm{C} \mathrm{NMR}\left(151 \mathrm{MHz}, \mathrm{CDCl}_{3}\right)$ $\delta$ 172.0, 169.2, 164.9, 153.6, 132.1, 125.7, 123.8, 121.3, 112.9, 67.9, 52.0, 51.8, 38.9, 36.9, 31.4, 25.2, 24.4, 22.6, 22.5, 19.4, 18.7. HRMS(ESI): calcd for $\mathrm{C}_{21} \mathrm{H}_{32} \mathrm{~N}_{2} \mathrm{O}_{5}: 393.2384$ (M $\left.+\mathrm{H}^{+}\right)$, found: $393.2382\left(\mathrm{M}+\mathrm{H}^{+}\right)$. 
Compound 14. To a solution of compound $10(2.00 \mathrm{~g}, 7.54 \mathrm{mmol})$ in $20 \mathrm{~mL}$ dichloromethane, HBTU (3.43 g, $9.05 \mathrm{mmol})$, HOBt $(1.22 \mathrm{~g}, 9.05 \mathrm{mmol})$ and $\mathrm{N}, \mathrm{N}$ diisopropyl-ethylamin $(2.43 \mathrm{~g}, 18.85 \mathrm{mmol})$ was added at $0{ }^{\circ} \mathrm{C} .30$ min later, the compound $6(1.15 \mathrm{~g}, 7.54 \mathrm{mmol})$ in $15 \mathrm{~mL}$ dichloromethane was added to the mixture. Then the reaction was allowed to proceed for $3 \mathrm{~h}$ at room temperature and inert atmosphere. The mixture was washed with diluted $\mathrm{HCl}$ and saturated brine. The combined organic layers were dried with sodium sulfate. The combined organic layers were dried with sodium sulfate, and solvent was removed in vacuum. The crude product was purified by column chromatography (petroleum ether/acetone $=5 / 1)$ to afford a white solid $(1.83 \mathrm{~g}, 67 \%) .{ }^{1} \mathrm{H}$ NMR (400 MHz, Chloroform- $d$ ) $\delta 8.34(\mathrm{~d}, J=8.1 \mathrm{~Hz}, 1 \mathrm{H}), 8.12(\mathrm{dd}, J=9.0,2.8 \mathrm{~Hz}, 1 \mathrm{H})$, $7.84(\mathrm{dd}, J=2.9,1.5 \mathrm{~Hz}, 1 \mathrm{H}), 7.77(\mathrm{~s}, 1 \mathrm{H}), 6.93(\mathrm{~d}, J=8.9 \mathrm{~Hz}, 1 \mathrm{H}), 4.65-4.47(\mathrm{~m}, 1 \mathrm{H})$, $4.17-4.07$ (m, 2H), 3.67 (s, 3H), $2.66(\mathrm{dd}, J=15.6,6.4 \mathrm{~Hz}, 1 \mathrm{H}), 2.54$ (dd, $J=15.6,6.4$ $\mathrm{Hz}, 1 \mathrm{H}), 2.16$ (s, 3H), $1.93-1.63(\mathrm{~m}, 3 \mathrm{H}), 1.31$ (d, $J=6.7 \mathrm{~Hz}, 6 \mathrm{H}), 1.04-0.88$ (m, 6H). ${ }^{13} \mathrm{C}$ NMR $\left(151 \mathrm{MHz}, \mathrm{CDCl}_{3}\right) \delta 170.0,165.7,153.7,136.7,131.5,129.5,128.6,127.0$, $125.4,123.9,121.6,113.0,68.0,39.0,38.0,31.7,28.4,26.7,25.5,22.7,22.6,14.2$. ESIMS: calcd for $\mathrm{C}_{19} \mathrm{H}_{28} \mathrm{~N}_{2} \mathrm{O}_{5}\left(\mathrm{M}+\mathrm{H}^{+}\right) 365.21$ found 365.66.

Compound 15. The compound $11(2.0 \mathrm{~g}, 5.7 \mathrm{mmol})$ was dissolved in $\mathrm{MeOH}(100 \mathrm{~mL})$, to which $2 \mathrm{~N} \mathrm{NaOH}$ (11.4 mL, $22.8 \mathrm{mmol}$ ) was added. The mixture was heated under reflux in oil bath for $2 \mathrm{~h}$. After removed the $\mathrm{MeOH}$ under vacuum, the aqueous layer was acidulated by addition of $3 \mathrm{~N} \mathrm{HCl}$ to $\mathrm{pH} 2$ followed by filtration to obtain a white solid (1.3 g, 66\%). ${ }^{1} \mathrm{H}$ NMR (600 MHz, DMSO- $\left.d_{6}\right) \delta 12.33(\mathrm{~s}, 1 \mathrm{H}), 9.93(\mathrm{~s}, 1 \mathrm{H}), 8.33(\mathrm{t}, J=5.8 \mathrm{~Hz}$, $1 \mathrm{H}), 7.96(\mathrm{~d}, J=2.8 \mathrm{~Hz}, 1 \mathrm{H}), 7.76(\mathrm{dd}, J=9.0,2.8 \mathrm{~Hz}, 1 \mathrm{H}), 7.10(\mathrm{~d}, J=9.0 \mathrm{~Hz}, 1 \mathrm{H}), 4.09$ (t, $J=6.6 \mathrm{~Hz}, 2 \mathrm{H}), 3.48$ (q, $J=6.3 \mathrm{~Hz}, 2 \mathrm{H}), 2.48(\mathrm{~d}, J=6.5 \mathrm{~Hz}, 2 \mathrm{H}), 2.00(\mathrm{~s}, 3 \mathrm{H}), 1.81$ $1.71(\mathrm{~m}, 1 \mathrm{H}), 1.71-1.61(\mathrm{~m}, 2 \mathrm{H}), 0.93(\mathrm{~d}, J=6.6 \mathrm{~Hz}, 6 \mathrm{H}) .{ }^{13} \mathrm{C}$ NMR $(151 \mathrm{MHz}$, DMSO$\left.d_{6}\right) \delta 173.2,168.0,164.3,152.2,132.6,123.2,122.0,121.6,113.4,67.5,37.1,35.0,33.8$, 24.7, 23.8, 22.4. $\mathrm{HRMS}(\mathrm{ESI})$ : calcd for $\mathrm{C}_{17} \mathrm{H}_{24} \mathrm{~N}_{2} \mathrm{O}_{5}: 335.1612\left(\mathrm{M}-\mathrm{H}^{+}\right.$), found: 335.1610 $\left(\mathrm{M}-\mathrm{H}^{+}\right)$.

Compound 16. The compound $12(2.0 \mathrm{~g}, 4.5 \mathrm{mmol})$ was dissolved in $\mathrm{MeOH}(100 \mathrm{~mL})$, to which $2 \mathrm{~N} \mathrm{NaOH}(9.1 \mathrm{~mL}, 18.2 \mathrm{mmol})$ was added. The mixture was heated under reflux in oil bath for $2 \mathrm{~h}$. After removed the $\mathrm{MeOH}$ under vacuum, the aqueous layer was acidulated by addition of $3 \mathrm{~N} \mathrm{HCl}$ to $\mathrm{pH} 2$ followed by filtration to obtain a white solid. The solid was washed by methanol to get a white solid $(1.5 \mathrm{~g}, 78 \%) .{ }^{1} \mathrm{H}$ NMR $(400 \mathrm{MHz}$, DMSO- $\left.d_{6}\right) \delta 8.37(\mathrm{~d}, J=3.0 \mathrm{~Hz}, 1 \mathrm{H}), 8.28(\mathrm{dd}, J=9.2,3.0 \mathrm{~Hz}, 1 \mathrm{H}), 8.26(\mathrm{~s}, 1 \mathrm{H}), 8.24(\mathrm{~s}$, $1 \mathrm{H}), 7.34(\mathrm{~d}, J=9.2 \mathrm{~Hz}, 1 \mathrm{H}), 7.29-7.22(\mathrm{~m}, 2 \mathrm{H}), 7.20-7.14(\mathrm{~m}, 3 \mathrm{H}), 4.49(\mathrm{q}, J=6.9$ $\mathrm{Hz}, 1 \mathrm{H}), 4.20(\mathrm{t}, J=6.5 \mathrm{~Hz}, 2 \mathrm{H}), 2.91-2.79(\mathrm{~m}, 2 \mathrm{H}), 2.53-2.49(\mathrm{~m}, 1 \mathrm{H}), 2.40(\mathrm{~m}, 1 \mathrm{H})$, $2.18(\mathrm{~s}, 3 \mathrm{H}), 1.74-1.45(\mathrm{~m}, 3 \mathrm{H}), 0.86(\mathrm{t}, J=6.3 \mathrm{~Hz}, 6 \mathrm{H}) .{ }^{13} \mathrm{C}$ NMR $(151 \mathrm{MHz}$, DMSO$\left.d_{6}\right) \delta 172.4,172.0,162.5,161.2,140.4,138.1,129.2,128.3,127.6,126.4,125.8,123.9$, 113.8, 68.4, 48.6, 47.7, 36.7, 24.4, 22.3, 22.2, 22.0, 21.1. ESI-MS: calcd for $\mathrm{C}_{24} \mathrm{H}_{30} \mathrm{~N}_{2} \mathrm{O}_{5}$ $\left(\mathrm{M}+\mathrm{Na}^{+}\right) 449.20$ found 449.95 . 
Compound 17. The compound 13 (3.0 g, $7.7 \mathrm{mmol})$ was dissolved in $\mathrm{MeOH}(100 \mathrm{~mL})$, to which $2 \mathrm{~N} \mathrm{NaOH}(15.1 \mathrm{~mL}, 30.2 \mathrm{mmol})$ was added. The mixture was heated under reflux in oil bath for $2 \mathrm{~h}$. After removed the $\mathrm{MeOH}$ under vacuum, the aqueous layer was acidulated by addition of $3 \mathrm{~N} \mathrm{HCl}$ to $\mathrm{pH} 2$ followed by filtration to obtain a white solid (2.3 g, 80\%). ${ }^{1} \mathrm{H}$ NMR $\left(600 \mathrm{MHz}\right.$, Methanol- $\left.d_{4}\right) \delta 8.06(\mathrm{~d}, J=2.8 \mathrm{~Hz}, 1 \mathrm{H}), 7.53(\mathrm{dd}, J=8.8$, $2.8 \mathrm{~Hz}, 1 \mathrm{H}), 6.98$ (d, $J=8.8 \mathrm{~Hz}, 1 \mathrm{H}), 4.38-4.33(\mathrm{~m}, 1 \mathrm{H}), 4.17-4.05$ (m, 2H), 2.41 (dd, $J=14.9,5.2 \mathrm{~Hz}, 1 \mathrm{H}), 2.28(\mathrm{dd}, J=14.9,8.4 \mathrm{~Hz}, 1 \mathrm{H}), 2.07(\mathrm{~s}, 3 \mathrm{H}), 1.88-1.65(\mathrm{~m}, 4 \mathrm{H})$, $0.93(\mathrm{t}, J=6.8 \mathrm{~Hz}, 12 \mathrm{H}) .{ }^{13} \mathrm{C}$ NMR $\left(151 \mathrm{MHz}\right.$, Methanol- $\left.d_{4}\right) \delta 180.2,172.1,167.6,154.7$, $126.9,125.5,123.4,114.2,69.1,54.4,42.5,39.4,33.6,26.4,23.1,20.3,19.0$. ESI-MS: calcd for $\mathrm{C}_{20} \mathrm{H}_{30} \mathrm{~N}_{2} \mathrm{O}_{5}: 379.22\left(\mathrm{M}+\mathrm{H}^{+}\right)$, found: $379.75\left(\mathrm{M}+\mathrm{H}^{+}\right)$.

Compound 18. The compound $14(2.0 \mathrm{~g}, 5.5 \mathrm{mmol})$ was dissolved in $\mathrm{MeOH}(100 \mathrm{~mL})$, to which $2 \mathrm{~N} \mathrm{NaOH}(11.0 \mathrm{~mL}, 22.0 \mathrm{mmol})$ was added. The mixture was heated under reflux in oil bath for $2 \mathrm{~h}$. After removed the $\mathrm{MeOH}$ under vacuum, the aqueous layer was acidulated by addition of $3 \mathrm{~N} \mathrm{HCl}$ to $\mathrm{pH} 2$ followed by filtration to obtain a white solid (1.3 g, 68\%). 78\%. ${ }^{1} \mathrm{H}$ NMR (600 MHz, DMSO- $\left.d_{6}\right) \delta 12.25$ (s, 1H), 9.89 (s, 1H), 8.20 (d, $J=$ $8.0 \mathrm{~Hz}, 1 \mathrm{H}), 7.92(\mathrm{~d}, J=2.9 \mathrm{~Hz}, 1 \mathrm{H}), 7.72(\mathrm{dd}, J=8.9,2.8 \mathrm{~Hz}, 1 \mathrm{H}), 7.04(\mathrm{~d}, J=9.0 \mathrm{~Hz}$, $1 \mathrm{H}), 4.29$ (p, $J=6.7 \mathrm{~Hz}, 1 \mathrm{H}), 4.04$ (t, $J=6.4 \mathrm{~Hz}, 2 \mathrm{H}), 2.58-2.33(\mathrm{~m}, 2 \mathrm{H}), 1.98(\mathrm{~s}, 3 \mathrm{H})$, $1.83-1.59(\mathrm{~m}, 3 \mathrm{H}), 1.17(\mathrm{~d}, J=6.7 \mathrm{~Hz}, 3 \mathrm{H}), 0.89(\mathrm{t}, J=6.4 \mathrm{~Hz}, 6 \mathrm{H}) .{ }^{13} \mathrm{C}$ NMR $(151 \mathrm{MHz}$, DMSO- $\left.d_{6}\right) \delta 172.5,168.0,163.6,152.2,132.6,123.1,122.3,121.6,113.3,67.2,42.0,40.2$, 39.5, 37.2, 24.6, 23.8, 22.4, 22.3, 19.9. HRMS (ESI): calcd for $\mathrm{C}_{18} \mathrm{H}_{26} \mathrm{~N}_{2} \mathrm{O}_{5}: 351.1914$ (M $\left.+\mathrm{H}^{+}\right)$, found $351.1912\left(\mathrm{M}+\mathrm{H}^{+}\right)$.

Compound 19 was synthesized according to previously reported procedures. ${ }^{3}$

Compound 20. To a solution of compound $19(2.00 \mathrm{~g}, 7.91 \mathrm{mmol})$ in $20 \mathrm{~mL}$ dichloromethane, HBTU (3.60 g, $9.49 \mathrm{mmol})$, HOBt (1.28 g, $9.49 \mathrm{mmol})$ and $N, N$ diisopropyl-ethylamin $(2.45 \mathrm{~g}, 18.98 \mathrm{mmol})$ was added at $0{ }^{\circ} \mathrm{C} .30 \mathrm{~min}$ later, methylamine hydrochloride $(0.53 \mathrm{~g}, 7.91 \mathrm{mmol})$ in $15 \mathrm{~mL}$ dichloromethane was added to the mixture. Then the reaction was allowed to proceed for $3 \mathrm{~h}$ at room temperature and inert atmosphere. The mixture was washed with diluted $\mathrm{HCl}$ and saturated brine, and solvent was removed in vacuum. The crude product was purified by column chromatography (petroleum ether/acetone $=6 / 1)$ to afford a white solid $(1.45 \mathrm{~g}, 69 \%) .{ }^{1} \mathrm{H} \mathrm{NMR}\left(600 \mathrm{MHz}, \mathrm{CDCl}_{3}\right) \delta$ $9.10(\mathrm{~d}, J=3.0 \mathrm{~Hz}, 1 \mathrm{H}), 8.30(\mathrm{dd}, J=9.1,3.0 \mathrm{~Hz}, 1 \mathrm{H}), 7.74(\mathrm{~s}, 1 \mathrm{H}), 7.07$ (d, $J=9.1 \mathrm{~Hz}$, $1 \mathrm{H}), 4.27(\mathrm{t}, J=6.3 \mathrm{~Hz}, 2 \mathrm{H}), 3.03(\mathrm{~d}, J=4.8 \mathrm{~Hz}, 3 \mathrm{H}), 1.96-1.76(\mathrm{~m}, 3 \mathrm{H}), 1.03(\mathrm{~d}, J=6.4$ $\mathrm{Hz}, 6 \mathrm{H}) .{ }^{13} \mathrm{C} \mathrm{NMR}\left(151 \mathrm{MHz}, \mathrm{CDCl}_{3}\right) \delta 163.9,161.2,142.0,128.6,128.0,122.7,112.6$, 69.0, 37.7, 26.9, 25.5, 22.7. HRMS(ESI): calcd for $\mathrm{C}_{13} \mathrm{H}_{18} \mathrm{~N}_{2} \mathrm{O}_{4}: 267.1339\left(\mathrm{M}+\mathrm{H}^{+}\right)$, found $267.1341\left(\mathrm{M}+\mathrm{H}^{+}\right)$. 
Compound 21. Compound 20 (3.00 g, $11.28 \mathrm{mmol})$ was reduced by catalytic hydrogenation in dichloromethane $(50 \mathrm{~mL})$ at room temperature and atmospheric pressure, using Pd-C $(0.30 \mathrm{~g}, 10 \%)$ as the catalyst. Removal of catalyst. After all substrate was reduced, removal of catalyst and solvent gave the product as yellow oil. This compound was used without further purification.

Compound 22. To a solution of Boc-glycine (2.36 g, $13.47 \mathrm{mmol})$ in $30 \mathrm{~mL}$ dichloromethane, HBTU (5.11 g, $13.47 \mathrm{mmol})$, HOBt $(1.82 \mathrm{~g}, 13.47 \mathrm{mmol})$ and $\mathrm{N}, \mathrm{N}$ diisopropyl- ethylamine $(3.63 \mathrm{~g}, 28.06 \mathrm{mmol})$ was added at $0{ }^{\circ} \mathrm{C} .30 \mathrm{~min}$ later, the compound 21 ( $2.65 \mathrm{~g}, 11.22 \mathrm{mmol})$ in $15 \mathrm{~mL}$ dichloromethane was added to the mixture. Then the reaction was allowed to proceed for $8 \mathrm{~h}$ at room temperature and inert atmosphere. The mixture was washed with diluted $\mathrm{HCl}$ and saturated brine, and solvent was removed in vacuum. Purification by flash column chromatography (petroleum ether/ethyl acetate $=$ 2/1) to afford a white solid (3.09 g, 70\%). ${ }^{1} \mathrm{H}$ NMR $\left(400 \mathrm{MHz}\right.$, DMSO- $\left.d_{6}\right) \delta 9.90(\mathrm{~s}, 1 \mathrm{H})$, $8.16-8.02(\mathrm{~m}, 1 \mathrm{H}), 8.01-7.88(\mathrm{~m}, 1 \mathrm{H}), 7.74(\mathrm{~d}, J=8.9 \mathrm{~Hz}, 1 \mathrm{H}), 7.08(\mathrm{~d}, J=9.0 \mathrm{~Hz}, 1 \mathrm{H})$, $7.01-6.98(\mathrm{~m}, 1 \mathrm{H}), 4.08(\mathrm{t}, J=6.7 \mathrm{~Hz}, 2 \mathrm{H}), 3.71(\mathrm{~d}, J=6.1 \mathrm{~Hz}, 2 \mathrm{H}), 2.82(\mathrm{~d}, J=4.7 \mathrm{~Hz}$, $3 \mathrm{H}), 1.78-1.60$ (m, 3H), 1.39 (s, 9H), 0.92 (d, $J=6.6 \mathrm{~Hz}, 6 \mathrm{H}) .{ }^{13} \mathrm{C}$ NMR $(101 \mathrm{MHz}$, DMSO- $\left.d_{6}\right) \delta 168.0,165.2,156.0,152.2,132.2,123.0,121.6,113.5,78.1,67.5,43.8,37.24$, 28.2, 26.2, 24.9, 22.4. ESI-MS: calcd for $\mathrm{C}_{20} \mathrm{H}_{31} \mathrm{~N}_{3} \mathrm{O}_{5}\left(\mathrm{M}+\mathrm{Na}^{+}\right) 416.47$ found 416.08.

Compound 23. To a solution of Boc-alanine $(2.55 \mathrm{~g}, 13.47 \mathrm{mmol})$ in $30 \mathrm{~mL}$ dichloromethane, HBTU $(5.11 \mathrm{~g}, 13.47 \mathrm{mmol})$, HOBt $(1.82 \mathrm{~g}, 13.47 \mathrm{mmol})$ and $N, N$ diisopropyl- ethylamine $(3.63 \mathrm{~g}, 28.06 \mathrm{mmol})$ was added at $0{ }^{\circ} \mathrm{C} .30 \mathrm{~min}$ later, the compound 21 ( $2.65 \mathrm{~g}, 11.22 \mathrm{mmol})$ in $15 \mathrm{~mL}$ dichloromethane was added to the mixture. Then the reaction was allowed to proceed for $8 \mathrm{~h}$ at room temperature and inert atmosphere. The mixture was washed with diluted $\mathrm{HCl}$ and saturated brine, and solvent was removed in vacuum. Purification by flash column chromatography (petroleum ether/ethyl acetate $=$ $2 / 1)$ to afford a white solid $(3.47 \mathrm{~g}, 76 \%) .{ }^{1} \mathrm{H}$ NMR $\left(400 \mathrm{MHz}, \mathrm{DMSO}-d_{6}\right) \delta 9.89(\mathrm{~s}, 1 \mathrm{H})$, $8.12-8.01(\mathrm{~m}, 1 \mathrm{H}), 8.01-7.91(\mathrm{~m}, 1 \mathrm{H}), 7.74(\mathrm{~d}, J=9.0 \mathrm{~Hz}, 1 \mathrm{H}), 7.09(\mathrm{~d}, J=8.9 \mathrm{~Hz}, 1 \mathrm{H})$, $7.00(\mathrm{~d}, J=7.4 \mathrm{~Hz}, 1 \mathrm{H}), 4.17-3.98(\mathrm{~m}, 3 \mathrm{H}), 2.81(\mathrm{~d}, J=4.7 \mathrm{~Hz}, 3 \mathrm{H}), 1.80-1.57(\mathrm{~m}, 3 \mathrm{H})$, $1.49-1.12(\mathrm{~m}, 12 \mathrm{H}), 0.93(\mathrm{~d}, J=6.5 \mathrm{~Hz}, 6 \mathrm{H}) .{ }^{13} \mathrm{C}$ NMR $\left(101 \mathrm{MHz}, \mathrm{DMSO}-d_{6}\right) \delta 171.5$, 165.1, 155.2, 152.1, 132.3, 122.9, 121.6, 113.4, 78.0, 67.4, 50.4, 37.2, 28.2, 26.1, 24.8, 22.4, 18.1. ESI-MS: calcd for $\mathrm{C}_{21} \mathrm{H}_{31} \mathrm{~N}_{3} \mathrm{O}_{5}\left(\mathrm{M}+\mathrm{Na}^{+}\right) 430.23$ found 430.38 .

Compound 24. To a solution of Boc-phenylalanine (3.57 g, $13.47 \mathrm{mmol})$ in $30 \mathrm{~mL}$ dichloromethane, HBTU $(5.11 \mathrm{~g}, 13.47 \mathrm{mmol})$, HOBt $(1.82 \mathrm{~g}, 13.47 \mathrm{mmol})$ and $\mathrm{N}, \mathrm{N}$ diisopropyl- ethylamine $(3.63 \mathrm{~g}, 28.06 \mathrm{mmol})$ was added at $0{ }^{\circ} \mathrm{C} .30 \mathrm{~min}$ later, the compound 21 ( $2.65 \mathrm{~g}, 11.22 \mathrm{mmol})$ in $15 \mathrm{~mL}$ dichloromethane was added to the mixture. Then the reaction was allowed to proceed for $8 \mathrm{~h}$ at room temperature and inert atmosphere. The mixture was washed with diluted $\mathrm{HCl}$ and saturated brine, and solvent was removed in vacuum. The crude product was purified by column chromatography (petroleum 
ether/acetone $=6 / 1)$ to afford a white solid $(3.58 \mathrm{~g}, 66 \%) .{ }^{1} \mathrm{H}$ NMR $(400 \mathrm{MHz}$, Chloroformd) $\delta 8.02(\mathrm{~m}, 2 \mathrm{H}), 7.75-7.67(\mathrm{~m}, 2 \mathrm{H}), 7.35-7.26(\mathrm{~m}, 3 \mathrm{H}), 7.27-7.18(\mathrm{~m}, 2 \mathrm{H}), 6.94(\mathrm{~d}$, $J=9.0 \mathrm{~Hz}, 1 \mathrm{H}), 5.18-4.91(\mathrm{~m}, 1 \mathrm{H}), 4.53-4.36(\mathrm{~m}, 1 \mathrm{H}), 4.13(\mathrm{t}, J=6.4 \mathrm{~Hz}, 2 \mathrm{H}), 3.15(\mathrm{~d}$, $J=6.7 \mathrm{~Hz}, 2 \mathrm{H}), 2.99(\mathrm{~d}, J=4.8 \mathrm{~Hz}, 3 \mathrm{H}), 1.78(\mathrm{~m}, 3 \mathrm{H}), 1.42(\mathrm{~s}, 9 \mathrm{H}), 1.00(\mathrm{~d}, J=6.1 \mathrm{~Hz}$, $6 \mathrm{H}) .{ }^{13} \mathrm{C}$ NMR $\left(151 \mathrm{MHz}, \mathrm{CDCl}_{3}\right) \delta 171.6,169.2,164.5,153.3,132.4,125.5,123.5,121.2$, $112.7,67.7,51.6,42.4,40.3,37.8,25.0,24.0,22.5,22.3,20.1$. ESI-MS: calcd for $\mathrm{C}_{27} \mathrm{H}_{37} \mathrm{~N}_{3} \mathrm{O}_{5}\left(\mathrm{M}+\mathrm{Na}^{+}\right) 506.26$ found 506.25.

Compound 25. To a solution of Boc-valine (2.93 g, $13.47 \mathrm{mmol})$ in $30 \mathrm{~mL}$ dichloromethane, HBTU (5.11 g, $13.47 \mathrm{mmol})$, HOBt $(1.82 \mathrm{~g}, 13.47 \mathrm{mmol})$ and $\mathrm{N}, \mathrm{N}$ diisopropyl- ethylamine $(3.63 \mathrm{~g}, 28.06 \mathrm{mmol})$ was added at $0{ }^{\circ} \mathrm{C} .30 \mathrm{~min}$ later, the compound 21 ( $2.65 \mathrm{~g}, 11.22 \mathrm{mmol})$ in $15 \mathrm{~mL}$ dichloromethane was added to the mixture. Then the reaction was allowed to proceed for $8 \mathrm{~h}$ at room temperature and inert atmosphere. The mixture was washed with diluted $\mathrm{HCl}$ and saturated brine, and solvent was removed in vacuum. The crude product was purified by column chromatography (petroleum ether/acetone $=6 / 1)$ to afford a white solid $(3.91 \mathrm{~g}, 80 \%) .{ }^{1} \mathrm{H}$ NMR $(400 \mathrm{MHz}$, Chloroformd) $\delta 9.04-8.63(\mathrm{~m}, 1 \mathrm{H}), 8.12-8.05(\mathrm{~m}, 1 \mathrm{H}), 8.05-7.98(\mathrm{~m}, 1 \mathrm{H}), 7.95(\mathrm{~d}, J=8.7 \mathrm{~Hz}$, $1 \mathrm{H}), 7.18-7.05(\mathrm{~m}, 1 \mathrm{H}), 6.92(\mathrm{~d}, J=8.7 \mathrm{~Hz}, 1 \mathrm{H}), 4.18-4.02(\mathrm{~m}, 3 \mathrm{H}), 3.00(\mathrm{~d}, J=4.6$ $\mathrm{Hz}, 3 \mathrm{H}), 1.86-1.69$ (m, 3H), 1.49 (d, $J=6.1 \mathrm{~Hz}, 3 \mathrm{H}), 1.46-1.40(\mathrm{~m}, 1 \mathrm{H}), 1.37$ (s, 9H), $1.23(\mathrm{~d}, J=6.6 \mathrm{~Hz}, 3 \mathrm{H}), 0.99(\mathrm{~d}, J=6.1 \mathrm{~Hz}, 6 \mathrm{H}) .{ }^{13} \mathrm{C} \mathrm{NMR}\left(101 \mathrm{MHz}, \mathrm{CDCl}_{3}\right) \delta 172.2$, $167.9,166.1,155.7,153.7,131.9,125.3,123.7,121.5,113.2,79.4,68.2,55.1,44.6,44.2$, 43.1, 38.1, 28.5, 26.8, 25.6, 22.7, 21.0, 18.8, 17.5, 12.5. ESI-MS: calcd for $\mathrm{C}_{23} \mathrm{H}_{37} \mathrm{~N}_{3} \mathrm{O}_{5}$ $\left(\mathrm{M}+\mathrm{Na}^{+}\right) 458.26$ found 458.27 .

Compound 26. To a solution of Boc-methionine (3.36 g, $13.47 \mathrm{mmol})$ in $30 \mathrm{~mL}$ dichloromethane, HBTU $(5.11 \mathrm{~g}, 13.47 \mathrm{mmol})$, HOBt $(1.82 \mathrm{~g}, 13.47 \mathrm{mmol})$ and $N, N-$ diisopropyl- ethylamine $(3.63 \mathrm{~g}, 28.06 \mathrm{mmol})$ was added at $0{ }^{\circ} \mathrm{C} .30 \mathrm{~min}$ later, the compound 21 ( $2.65 \mathrm{~g}, 11.22 \mathrm{mmol})$ in $15 \mathrm{~mL}$ dichloromethane was added to the mixture. Then the reaction was allowed to proceed for $8 \mathrm{~h}$ at room temperature and inert atmosphere. The mixture was washed with diluted $\mathrm{HCl}$ and saturated brine, and solvent was removed in vacuum. The crude product was purified by column chromatography (petroleum ether/acetone $=6 / 1)$ to afford a white solid $(3.46 \mathrm{~g}, 66 \%) .{ }^{1} \mathrm{H}$ NMR $(400 \mathrm{MHz}$, Chloroformd) $\delta 8.50-8.35(\mathrm{~m}, 1 \mathrm{H}), 8.11-7.95(\mathrm{~m}, 3 \mathrm{H}), 7.91(\mathrm{dd}, J=2.9,1.4 \mathrm{~Hz}, 1 \mathrm{H}), 6.93(\mathrm{~d}, J=$ $9.1 \mathrm{~Hz}, 1 \mathrm{H}), 5.31-5.19(\mathrm{~m}, 1 \mathrm{H}), 4.12(\mathrm{t}, J=7.2,5.6 \mathrm{~Hz}, 2 \mathrm{H}), 2.99$ (d, $J=4.8,1.3 \mathrm{~Hz}$, $3 \mathrm{H}), 2.68-2.50(\mathrm{~m}, 2 \mathrm{H}), 2.23-2.14(\mathrm{~m}, 1 \mathrm{H}), 2.10(\mathrm{~s}, 3 \mathrm{H}), 2.06-1.94(\mathrm{~m}, 1 \mathrm{H}), 1.87-$ $1.63(\mathrm{~m}, 3 \mathrm{H}), 1.45(\mathrm{~s}, 9 \mathrm{H}), 1.04-0.93(\mathrm{~m}, 6 \mathrm{H}) \cdot{ }^{13} \mathrm{C} \mathrm{NMR}\left(101 \mathrm{MHz}, \mathrm{CDCl}_{3}\right) \delta 170.5$, 165.9, 155.8, 153.5, 131.7, 125.1, 123.7, 123.6, 121.4, 113.0, 80.0, 67.9, 54.2, 37.9, 32.5, 30.1, 28.3, 26.6, 26.6, 25.4, 22.6, 15.3. ESI-MS: calcd for $\mathrm{C}_{24} \mathrm{H}_{39} \mathrm{~N}_{3} \mathrm{O}_{5} \mathrm{~S}\left(\mathrm{M}+\mathrm{Na}^{+}\right) 490.23$ found 490.25 . 
Compound 27. To a solution of Boc-D-phenylalanine (3.57 g, $13.47 \mathrm{mmol})$ in $30 \mathrm{~mL}$ dichloromethane, HBTU $(5.11 \mathrm{~g}, 13.47 \mathrm{mmol})$, HOBt $(1.82 \mathrm{~g}, 13.47 \mathrm{mmol})$ and $\mathrm{N}, \mathrm{N}$ diisopropyl- ethylamine $(3.63 \mathrm{~g}, 28.06 \mathrm{mmol})$ was added at $0{ }^{\circ} \mathrm{C} .30 \mathrm{~min}$ later, the compound 21 ( $2.65 \mathrm{~g}, 11.22 \mathrm{mmol})$ in $15 \mathrm{~mL}$ dichloromethane was added to the mixture. Then the reaction was allowed to proceed for $8 \mathrm{~h}$ at room temperature and inert atmosphere. The mixture was washed with diluted $\mathrm{HCl}$ and saturated brine, and solvent was removed in vacuum. The crude product was purified by column chromatography (petroleum ether/acetone $=6 / 1)$ to afford a white solid $(4.12 \mathrm{~g}, 76 \%) .{ }^{1} \mathrm{H}$ NMR $\left(400 \mathrm{MHz}, \mathrm{DMSO}-d_{6}\right)$ $\delta 9.99(\mathrm{~s}, 1 \mathrm{H}), 8.05-8.00(\mathrm{~m}, 1 \mathrm{H}), 7.93-7.84(\mathrm{~m}, 1 \mathrm{H}), 7.68(\mathrm{~d}, \mathrm{~J}=8.9 \mathrm{~Hz}, 1 \mathrm{H}), 7.33-$ $7.01(\mathrm{~m}, 7 \mathrm{H}), 4.30-4.19(\mathrm{~m}, 1 \mathrm{H}), 4.06(\mathrm{t}, \mathrm{J}=6.6 \mathrm{~Hz}, 2 \mathrm{H}), 3.01-2.90(\mathrm{~m}, 1 \mathrm{H}), 2.76(\mathrm{~d}, \mathrm{~J}$ $=4.9 \mathrm{~Hz}, 3 \mathrm{H}), 2.64-2.56(\mathrm{~m}, 1 \mathrm{H}), 1.79-1.59(\mathrm{~m}, 3 \mathrm{H}), 1.43(\mathrm{~s}, 9 \mathrm{H}), 0.90(\mathrm{~d}, \mathrm{~J}=6.4 \mathrm{~Hz}$, $6 \mathrm{H}) .{ }^{13} \mathrm{C}$ NMR $\left(151 \mathrm{MHz}, \mathrm{CDCl}_{3}\right) \delta 171.5,169.1,164.4,153.2,132.3,125.5,123.5,121.1$, $112.69,67.7,51.5,42.4,40.3,37.7,24.9,24.0,22.5,22.3,20.1$. ESI-MS: calcd for $\mathrm{C}_{27} \mathrm{H}_{37} \mathrm{~N}_{3} \mathrm{O}_{5}\left(\mathrm{M}+\mathrm{Na}^{+}\right) 506.26$ found 506.16 .

General procedure for the synthesis of compounds $28,29,30,31,32$ and 33 . The compound 22, 23, 24, 25, 26 and $27(10.00 \mathrm{mmol})$ was treated with hydrochloric solution of ethyl acetic $(2 \mathrm{~N}, 30 \mathrm{~mL})$, then the mixture was stirred at room temperature for $1 \mathrm{~h}$. Removed the solvent to obtain compound $28,29,30,31,32$ and 33 as a white solid.

Compound 28. White solid (2.34 g, 71\%). ${ }^{1} \mathrm{H}$ NMR (600 MHz, DMSO- $\left.d_{6}\right) \delta 10.65$ (s, $1 \mathrm{H}), 8.20(\mathrm{~s}, 3 \mathrm{H}), 8.07$ (q, $J=4.7 \mathrm{~Hz}, 1 \mathrm{H}), 7.96(\mathrm{~d}, J=2.8 \mathrm{~Hz}, 1 \mathrm{H}), 7.71$ (dd, $J=8.9,2.8$ $\mathrm{Hz}, 1 \mathrm{H}), 7.15(\mathrm{~d}, J=9.0 \mathrm{~Hz}, 1 \mathrm{H}), 4.10$ (t, $J=6.6 \mathrm{~Hz}, 2 \mathrm{H}), 3.75(\mathrm{~s}, 2 \mathrm{H}), 2.80$ (d, $J=4.7$ $\mathrm{Hz}, 3 \mathrm{H}), 1.80-1.70(\mathrm{~m}, 1 \mathrm{H}), 1.70-1.60(\mathrm{~m}, 2 \mathrm{H}), 0.94(\mathrm{~d}, J=6.6 \mathrm{~Hz}, 6 \mathrm{H}) .{ }^{13} \mathrm{C}$ NMR $(151$ MHz, DMSO- $\left.d_{6}\right) \delta$ 165.0, 164.5, 152.5, 131.4, 123.3, 122.9, 121.5, 113.6, 67.4, 40.9, 37.2, 26.2, 24.8, 22.5. HRMS(ESI): calcd for $\mathrm{C}_{15} \mathrm{H}_{23} \mathrm{~N}_{3} \mathrm{O}_{3}: 294.1812\left(\mathrm{M}+\mathrm{H}^{+}\right)$, found: 294.1810 $\left(\mathrm{M}+\mathrm{H}^{+}\right)$.

Compound 29. White solid (2.61 g, 76\%). ${ }^{1} \mathrm{H}$ NMR (600 MHz, $\left.\mathrm{CDCl}_{3}\right) \delta 10.62-10.33$ $(\mathrm{m}, 1 \mathrm{H}), 8.57-8.20(\mathrm{~m}, 3 \mathrm{H}), 8.20-8.05(\mathrm{~m}, 1 \mathrm{H}), 8.05-7.87(\mathrm{~m}, 1 \mathrm{H}), 7.78-7.55(\mathrm{~m}$, $1 \mathrm{H}), 6.73-6.53(\mathrm{~m}, 1 \mathrm{H}), 4.70-4.50(\mathrm{~m}, 1 \mathrm{H}), 3.92-3.78(\mathrm{~m}, 2 \mathrm{H}), 2.92-2.76(\mathrm{~m}, 3 \mathrm{H})$, $1.21-1.16(\mathrm{~m}, 3 \mathrm{H}), 0.84(\mathrm{~d}, \mathrm{~J}=6.2 \mathrm{~Hz}, 6 \mathrm{H}), 0.78(\mathrm{t}, \mathrm{J}=7.0 \mathrm{~Hz}, 3 \mathrm{H}) .{ }^{13} \mathrm{C} \mathrm{NMR}(151 \mathrm{MHz}$, $\left.\mathrm{CDCl}_{3}\right) \delta 171.7,165.9,155.5,153.4,132.0,125.2,123.6,121.4,113.0,67.9,55.1,50.6$, 43.1, 37. 9, 26.7, 25.4, 22.6, 19.1, 12.6. HRMS(ESI): calcd for $\mathrm{C}_{16} \mathrm{H}_{25} \mathrm{~N}_{3} \mathrm{O}_{3}: 308.1696(\mathrm{M}$ $\left.+\mathrm{H}^{+}\right)$, found: $308.1691\left(\mathrm{M}+\mathrm{H}^{+}\right)$.

Compound 30. White solid (2.64 g, 63\%). ${ }^{1} \mathrm{H}$ NMR (600 MHz, DMSO- $\left.d_{6}\right) \delta 10.95(\mathrm{~s}, 1 \mathrm{H})$, $8.53(\mathrm{~s}, 3 \mathrm{H}), 8.06(\mathrm{q}, J=4.7 \mathrm{~Hz}, 1 \mathrm{H}), 7.92(\mathrm{~s}, 1 \mathrm{H}), 7.66(\mathrm{dd}, J=8.9,2.8 \mathrm{~Hz}, 1 \mathrm{H}), 7.33-$ $7.21(\mathrm{~m}, 5 \mathrm{H}), 7.12(\mathrm{~d}, J=8.8 \mathrm{~Hz}, 1 \mathrm{H}), 4.26(\mathrm{t}, J=6.9 \mathrm{~Hz}, 1 \mathrm{H}), 4.09(\mathrm{t}, J=6.6 \mathrm{~Hz}, 2 \mathrm{H})$, $3.23-3.06(\mathrm{~m}, 2 \mathrm{H}), 2.79(\mathrm{~d}, J=4.7 \mathrm{~Hz}, 3 \mathrm{H}), 1.77-1.68(\mathrm{~m}, 1 \mathrm{H}), 1.68-1.61(\mathrm{~m}, 2 \mathrm{H})$, $0.93(\mathrm{~d}, J=6.4 \mathrm{~Hz}, 6 \mathrm{H}) .{ }^{13} \mathrm{C}$ NMR $\left(151 \mathrm{MHz}, \mathrm{DMSO}-d_{6}\right) \delta 166.3,165.1,152.6,135.0$, 
131.2, 129.6, 128.5, 127.1, 123.3, 123.3, 121.8, 113.5, 67.4, 54.1, 37.2, 36.9, 26.2, 24.8, 22.5. HRMS(ESI): calcd for $\mathrm{C}_{22} \mathrm{H}_{29} \mathrm{~N}_{3} \mathrm{O}_{3}: 384.2282\left(\mathrm{M}+\mathrm{H}^{+}\right)$, found $384.2286\left(\mathrm{M}+\mathrm{H}^{+}\right)$.

Compound 31. White solid (2.56 g, 69\%). ${ }^{1} \mathrm{H}$ NMR $\left(600 \mathrm{MHz}\right.$, DMSO- $\left.d_{6}\right) \delta 10.73(\mathrm{~s}, 1 \mathrm{H})$, 8.27 (s, 3H), 8.07 (q, $J=4.9 \mathrm{~Hz}, 1 \mathrm{H}), 7.98$ (d, $J=2.8 \mathrm{~Hz}, 1 \mathrm{H}), 7.74$ (dd, $J=8.9,2.8 \mathrm{~Hz}$, $1 \mathrm{H}), 7.15(\mathrm{~d}, J=9.0 \mathrm{~Hz}, 1 \mathrm{H}), 4.11(\mathrm{t}, J=6.7 \mathrm{~Hz}, 2 \mathrm{H}), 3.78(\mathrm{~d}, J=6.1 \mathrm{~Hz}, 1 \mathrm{H}), 2.80(\mathrm{~d}, J$ $=4.7 \mathrm{~Hz}, 3 \mathrm{H}), 2.23-2.10(\mathrm{~m}, 1 \mathrm{H}), 1.80-1.70(\mathrm{~m}, 1 \mathrm{H}), 1.70-1.60(\mathrm{~m}, 2 \mathrm{H}), 0.99(\mathrm{~d}, J=$ $6.8 \mathrm{~Hz}, 6 \mathrm{H}), 0.94(\mathrm{~d}, J=6.6 \mathrm{~Hz}, 6 \mathrm{H}) .{ }^{13} \mathrm{C}$ NMR $\left(151 \mathrm{MHz}, \mathrm{DMSO}-d_{6}\right) \delta 166.5,165.0$, 152.7, 131.1, 123.4, 123.3, 121.8, 113.7, 67.5, 58.1, 38.2, 37.1, 29.9, 26.2, 24.8, 22.4, 18.4, 17.9. HRMS(ESI): calcd for $\mathrm{C}_{18} \mathrm{H}_{29} \mathrm{~N}_{3} \mathrm{O}_{3}: 336.2282\left(\mathrm{M}+\mathrm{H}^{+}\right)$, found: $336.2279\left(\mathrm{M}+\mathrm{H}^{+}\right)$.

Compound 32. White solid (2.66 g, 66\%). ${ }^{1} \mathrm{H}$ NMR $\left(600 \mathrm{MHz}, \mathrm{DMSO}-d_{6}\right) \delta 11.09(\mathrm{~s}, 1 \mathrm{H})$, 8.59 (s, 3H), 8.06 (q, $J=4.6 \mathrm{~Hz}, 1 \mathrm{H}), 8.02(\mathrm{~d}, J=2.7 \mathrm{~Hz}, 1 \mathrm{H}), 7.75$ (dd, $J=8.9,2.7 \mathrm{~Hz}$, $1 \mathrm{H}), 7.13(\mathrm{~d}, J=8.9 \mathrm{~Hz}, 1 \mathrm{H}), 4.12(\mathrm{t}, J=6.3 \mathrm{~Hz}, 1 \mathrm{H}), 4.08(\mathrm{t}, J=6.6 \mathrm{~Hz}, 2 \mathrm{H}), 2.79(\mathrm{~d}, J$ $=4.6 \mathrm{~Hz}, 3 \mathrm{H}), 2.61-2.52(\mathrm{~m}, 2 \mathrm{H}), 2.13(\mathrm{q}, J=7.6 \mathrm{~Hz}, 2 \mathrm{H}), 2.04(\mathrm{~s}, 3 \mathrm{H}), 1.77-1.67(\mathrm{~m}$, $1 \mathrm{H}), 1.65-1.61(\mathrm{~m}, 2 \mathrm{H}), 0.91(\mathrm{~d}, J=6.5 \mathrm{~Hz}, 6 \mathrm{H}) .{ }^{13} \mathrm{C}$ NMR $\left(151 \mathrm{MHz}\right.$, DMSO- $\left.d_{6}\right) \delta$ 166.6, 165.1, 152.6, 131.4, 123.4, 123.3, 121.8, 113.5, 67.4, 52.2, 37.2, 31.0, 28.4, 26.2, 24.8, 22.4, 14.5. HRMS(ESI): calcd for $\mathrm{C}_{18} \mathrm{H}_{29} \mathrm{~N}_{3} \mathrm{O}_{3} \mathrm{~S}: 368.2002\left(\mathrm{M}+\mathrm{H}^{+}\right)$, found: $368.2007\left(\mathrm{M}+\mathrm{H}^{+}\right)$.

Compound 33. White solid (2.94 g, 70\%). ${ }^{1} \mathrm{H}$ NMR $\left(600 \mathrm{MHz}\right.$, DMSO- $\left.d_{6}\right) \delta 10.82(\mathrm{~s}, 1 \mathrm{H})$, $8.47(\mathrm{~s}, 3 \mathrm{H}), 8.01(\mathrm{q}, J=4.7 \mathrm{~Hz}, 1 \mathrm{H}), 7.87(\mathrm{~d}, J=2.8 \mathrm{~Hz}, 1 \mathrm{H}), 7.61(\mathrm{dd}, J=8.9,2.8 \mathrm{~Hz}$, $1 \mathrm{H}), 7.31-7.16(\mathrm{~m}, 5 \mathrm{H}), 7.08(\mathrm{~d}, J=9.0 \mathrm{~Hz}, 1 \mathrm{H}), 4.24-4.16(\mathrm{~m}, 1 \mathrm{H}), 4.05(\mathrm{t}, J=6.6 \mathrm{~Hz}$, $2 \mathrm{H}), 3.21-3.06(\mathrm{~m}, 2 \mathrm{H}), 2.75(\mathrm{~d}, J=4.7 \mathrm{~Hz}, 3 \mathrm{H}), 1.84-1.45(\mathrm{~m}, 3 \mathrm{H}), 0.89(\mathrm{~d}, J=6.5$ $\mathrm{Hz}, 6 \mathrm{H}) .{ }^{13} \mathrm{C}$ NMR $\left(151 \mathrm{MHz}\right.$, DMSO- $\left.d_{6}\right) \delta 166.3,165.1,152.7,135.0,131.2,129.6,128.5$, 127.2, 123.4, 123.3, 121.9, 113.6, 67.5, 54.1, 39.5, 37.2, 36.9, 26.2, 24.8, 22.5. HRMS (ESI): calcd for $\mathrm{C}_{22} \mathrm{H}_{29} \mathrm{~N}_{3} \mathrm{O}_{3}: 384.2282\left(\mathrm{M}+\mathrm{H}^{+}\right)$, found $384.2284\left(\mathrm{M}+\mathrm{H}^{+}\right)$.

General procedure for the synthesis of compounds $2 \mathrm{a}, 2 \mathrm{~b}, 2 \mathrm{c}, 2 \mathrm{~d}, 2 \mathrm{e}, 2 \mathrm{f}, 2 \mathrm{~g}, 2 \mathrm{~h}, 2 \mathrm{i}$ and $\mathbf{2 j}$. To a solution of compound 15, 16, 17 and $18(1.12 \mathrm{mmol})$ in $30 \mathrm{~mL}$ dichloromethane, HBTU (511 mg, $1.35 \mathrm{mmol})$, HOBt $(182 \mathrm{mg}, 1.35 \mathrm{mmol})$ and $N$, $N$-diisopropylethylamine $\left(363 \mathrm{mg}, 2.81 \mathrm{mmol}\right.$ ) was added at $0{ }^{\circ} \mathrm{C} .30 \mathrm{~min}$ later, the compound $\mathbf{2 8 ,} \mathbf{2 9}$, 30, 31, 32 and $33(1.12 \mathrm{mmol})$ in $15 \mathrm{~mL}$ dichloromethane was added to the mixture. Then the reaction was allowed to proceed for $6 \mathrm{~h}$ at room temperature and inert atmosphere. The mixture was washed with diluted $\mathrm{HCl}$ and saturated brine, and solvent was removed in vacuum. The crude product was purified by column.

Compound 2a. Purification by flash column chromatography (petroleum ether/acetone $=$ 2/1) to afford a white solid (412 mg, 60\%). ${ }^{1} \mathrm{H}$ NMR $\left(400 \mathrm{MHz}, \mathrm{CDCl}_{3}\right) \delta 9.68(\mathrm{~s}, 1 \mathrm{H})$, $9.38(\mathrm{~s}, 1 \mathrm{H}), 8.68(\mathrm{t}, J=6.0 \mathrm{~Hz}, 1 \mathrm{H}), 8.33(\mathrm{dd}, J=9.0,2.4 \mathrm{~Hz}, 1 \mathrm{H}), 8.14(\mathrm{q}, J=5.0 \mathrm{~Hz}$, $1 \mathrm{H}), 8.08-8.04(\mathrm{~m}, 3 \mathrm{H}), 6.97(\mathrm{t}, J=5.7 \mathrm{~Hz}, 1 \mathrm{H}), 6.93(\mathrm{p}, J=9.6 \mathrm{~Hz}, 1 \mathrm{H}), 6.80(\mathrm{~d}, J=9.0$ 
$\mathrm{Hz}, 1 \mathrm{H}), 4.17-4.06(\mathrm{~m}, 6 \mathrm{H}), 3.82-3.74(\mathrm{~m}, 2 \mathrm{H}), 2.98(\mathrm{~d}, J=4.7 \mathrm{~Hz}, 3 \mathrm{H}), 2.60-2.51$ $(\mathrm{m}, 2 \mathrm{H}), 2.17(\mathrm{~s}, 3 \mathrm{H}), 1.87-1.74(\mathrm{~m}, 6 \mathrm{H}), 1.01(\mathrm{~d}, J=5.7 \mathrm{~Hz}, 12 \mathrm{H}) .{ }^{13} \mathrm{C} \mathrm{NMR}(151 \mathrm{MHz}$, DMSO- $\left.d_{6}\right) \delta 171.2,167.9,167.5,165.1,164.2,152.2,152.2,132.6,132.02,123.2,123.1$, 123.0, 122.1, 121.7, 121.6, 113.4, 113.4, 67.5, 67.4, 42.5, 37.2, 37.0, 35.5, 34.9, 26.2, 24.8, 24.7, 23.8, 22.4, 22.4. HRMS(ESI): calcd for $\mathrm{C}_{32} \mathrm{H}_{45} \mathrm{~N}_{5} \mathrm{O}_{7}: 612.3392\left(\mathrm{M}+\mathrm{H}^{+}\right)$, found: $612.3390\left(\mathrm{M}+\mathrm{H}^{+}\right)$.

Compound 2b. Purification by flash column chromatography (petroleum ether/acetone $=$ 2/1) to afford a white solid (393 mg, 56\%). ${ }^{1} \mathrm{H}$ NMR (400 MHz, $\left.\mathrm{CDCl}_{3}\right) \delta 9.46(\mathrm{~s}, 1 \mathrm{H})$, $9.39(\mathrm{~s}, 1 \mathrm{H}), 8.62(\mathrm{~s}, 1 \mathrm{H}), 8.31(\mathrm{~d}, J=6.7 \mathrm{~Hz}, 1 \mathrm{H}), 8.15(\mathrm{q}, J=4.1 \mathrm{~Hz}, 1 \mathrm{H}), 8.10-8.02$ $(\mathrm{m}, 2 \mathrm{H}), 8.01(\mathrm{~d}, J=2.5 \mathrm{~Hz}, 1 \mathrm{H}), 6.89(\mathrm{~d}, J=8.9 \mathrm{~Hz}, 1 \mathrm{H}), 6.86(\mathrm{~d}, J=7.8 \mathrm{~Hz}, 1 \mathrm{H}), 6.82$ $(\mathrm{d}, J=9.1 \mathrm{~Hz}, 1 \mathrm{H}), 4.71(\mathrm{p}, J=7.3 \mathrm{~Hz}, 1 \mathrm{H}), 4.14(\mathrm{t}, J=6.3 \mathrm{~Hz}, 2 \mathrm{H}), 4.13-4.03(\mathrm{~m}, 2 \mathrm{H})$, $3.87-3.77(\mathrm{~m}, 1 \mathrm{H}), 3.76-3.66(\mathrm{~m}, 1 \mathrm{H}), 2.98(\mathrm{~d}, J=4.9 \mathrm{~Hz}, 3 \mathrm{H}), 2.64-2.50(\mathrm{~m}, 2 \mathrm{H})$, $2.16(\mathrm{~s}, 3 \mathrm{H}), 1.92-1.68(\mathrm{~m}, 6 \mathrm{H}), 1.45(\mathrm{~d}, J=7.1 \mathrm{~Hz}, 3 \mathrm{H}), 1.05-0.93(\mathrm{~m}, 12 \mathrm{H}) .{ }^{13} \mathrm{C} \mathrm{NMR}$ $\left(101 \mathrm{MHz}, \mathrm{CDCl}_{3}\right) \delta 171.6,171.1,169.2,166.5,166.3,153.7,153.1,133.3,132.1,126.3$, $125.0,124.3,122.9,121.1,120.6,112.9,112.9,68.1,50.1,38.6,38.1,38.0,36.6,26.9$, 25.6, 24.5, 22.8, 22.7, 18.4. HRMS(ESI): calcd for $\mathrm{C}_{33} \mathrm{H}_{47} \mathrm{~N}_{5} \mathrm{O}_{7}: 626.3548\left(\mathrm{M}+\mathrm{H}^{+}\right)$, found: $626.3550\left(\mathrm{M}+\mathrm{H}^{+}\right)$.

Compound 2c. Purification by flash column chromatography (petroleum ether/acetone $=$ 2/1) to afford a white solid (378 mg, 48\%). ${ }^{1} \mathrm{H}$ NMR (400 MHz, $\left.\mathrm{CDCl}_{3}\right) \delta 9.54(\mathrm{~s}, 1 \mathrm{H})$, $9.14(\mathrm{~s}, 1 \mathrm{H}), 8.60(\mathrm{t}, J=6.4 \mathrm{~Hz}, 1 \mathrm{H}), 8.39(\mathrm{dd}, J=9.0,2.8 \mathrm{~Hz}, 1 \mathrm{H}), 8.21-8.13(\mathrm{~m}, 1 \mathrm{H})$, $8.07-7.98(\mathrm{~m}, 3 \mathrm{H}), 7.21-7.06(\mathrm{~m}, 5 \mathrm{H}), 6.95(\mathrm{~d}, J=9.6 \mathrm{~Hz}, 1 \mathrm{H}), 6.89(\mathrm{~d}, J=9.0 \mathrm{~Hz}, 1 \mathrm{H})$, $6.58(\mathrm{~d}, J=7.9 \mathrm{~Hz}, 1 \mathrm{H}), 4.98-4.86(\mathrm{~m}, 1 \mathrm{H}), 4.16-4.04(\mathrm{~m}, 4 \mathrm{H}), 3.74-3.57(\mathrm{~m}, 2 \mathrm{H})$, $3.21(\mathrm{~d}, J=6.1 \mathrm{~Hz}, 2 \mathrm{H}), 2.99(\mathrm{~d}, J=4.7 \mathrm{~Hz}, 3 \mathrm{H}), 2.56-2.48(\mathrm{~m}, 1 \mathrm{H}), 2.45-2.36(\mathrm{~m}, 1 \mathrm{H})$, $2.22(\mathrm{~s}, 3 \mathrm{H}), 1.85-1.73(\mathrm{~m}, 6 \mathrm{H}), 1.02(\mathrm{~d}, J=1.3 \mathrm{~Hz}, 6 \mathrm{H}), 1.00(\mathrm{~d}, J=1.3 \mathrm{~Hz}, 6 \mathrm{H}) .{ }^{13} \mathrm{C}$ NMR (151 MHz, $\left.\mathrm{CDCl}_{3}\right) \delta 171.8,170.1,169.1,166.6,166.3,153.8,153.2,133.4,132.0$, $126.5,125.0,124.4,122.9,121.2,120.5,113.0,112.9,68.2,53.9,38.7,38.1,38.0,36.6$, 31.5, 30.8, 26.9, 25.6, 25.6, 24.5, 22.8, 22.7, 15.6. HRMS(ESI): calcd for $\mathrm{C}_{39} \mathrm{H}_{51} \mathrm{~N}_{5} \mathrm{O}_{7}$ : $702.3861\left(\mathrm{M}+\mathrm{H}^{+}\right)$, found: $702.3868\left(\mathrm{M}+\mathrm{H}^{+}\right)$.

Compound 2d. Purification by flash column chromatography (petroleum ether/acetone = 1/1) to afford a white solid (484 mg, 66\%). ${ }^{1} \mathrm{H}$ NMR $\left(400 \mathrm{MHz}, \mathrm{CDCl}_{3}\right) \delta 9.42(\mathrm{~s}, 1 \mathrm{H})$, $9.23(\mathrm{~s}, 1 \mathrm{H}), 8.65(\mathrm{t}, J=6.3 \mathrm{~Hz}, 1 \mathrm{H}), 8.30(\mathrm{dd}, J=9.0,2.7 \mathrm{~Hz}, 1 \mathrm{H}), 8.15(\mathrm{q}, J=4.3 \mathrm{~Hz}$, $1 \mathrm{H}), 8.08-8.01(\mathrm{~m}, 3 \mathrm{H}), 6.98(\mathrm{~d}, J=9.0 \mathrm{~Hz}, 1 \mathrm{H}), 6.91(\mathrm{~d}, J=9.7 \mathrm{~Hz}, 1 \mathrm{H}), 6.85(\mathrm{~d}, J=$ $9.1 \mathrm{~Hz}, 1 \mathrm{H}), 4.58(\mathrm{dd}, J=8.9,5.3 \mathrm{~Hz}, 1 \mathrm{H}), 4.16-4.04(\mathrm{~m}, 4 \mathrm{H}), 3.84-3.73(\mathrm{~m}, 2 \mathrm{H}), 2.98$ (d, $J=4.8 \mathrm{~Hz}, 3 \mathrm{H}), 2.71-2.53(\mathrm{~m}, 2 \mathrm{H}), 2.39-2.35(\mathrm{~m}, 1 \mathrm{H}), 2.18(\mathrm{~s}, 3 \mathrm{H}), 1.89-1.72(\mathrm{~m}$, $6 \mathrm{H}), 1.02-0.92 \mathrm{~m}, 18 \mathrm{H}) .{ }^{13} \mathrm{C} \mathrm{NMR}\left(101 \mathrm{MHz}, \mathrm{CDCl}_{3}\right) \delta 171.9,170.3,169.2,166.7,166.4$, 153.8, 153.2, 133.2, 132.0, 126.7, 125.3, 124.4, 123.1, 121.2, 120.5, 113.0, 112. 9, 77.4, 77.2, 68.1, 59.5, 38.6, 38.1, 39.0, 36.6, 30.7, 26.9, 25.6, 25.6, 24.4, 22.8, 22.7, 19.6, 17.8 . HRMS(ESI): calcd for $\mathrm{C}_{35} \mathrm{H}_{51} \mathrm{~N}_{5} \mathrm{O}_{7}: 654.3861\left(\mathrm{M}+\mathrm{H}^{+}\right)$, found: $654.3858\left(\mathrm{M}+\mathrm{H}^{+}\right)$. 
Compound 2e. Purification by flash column chromatography (petroleum ether/acetone $=$ 2/1) to afford a white solid (537 mg, 70\%). ${ }^{1} \mathrm{H}$ NMR (400 MHz, $\left.\mathrm{CDCl}_{3}\right) \delta 9.49(\mathrm{~s}, 1 \mathrm{H})$, $9.43(\mathrm{~s}, 1 \mathrm{H}), 8.70-8.62(\mathrm{~m}, 1 \mathrm{H}), 8.34(\mathrm{~d}, J=8.4 \mathrm{~Hz}, 1 \mathrm{H}), 8.20-8.12(\mathrm{~m}, 1 \mathrm{H}), 8.08-8.00$ $(\mathrm{m}, 3 \mathrm{H}), 7.20-7.11(\mathrm{~m}, 1 \mathrm{H}), 6.90(\mathrm{~d}, J=8.4 \mathrm{~Hz}, 1 \mathrm{H}), 6.82(\mathrm{~d}, J=8.4 \mathrm{~Hz}, 1 \mathrm{H}), 4.79(\mathrm{q}, J$ $=8.2,7.5 \mathrm{~Hz}, 1 \mathrm{H}), 4.23-3.96(\mathrm{~m}, 4 \mathrm{H}), 3.86-3.68(\mathrm{~m}, 2 \mathrm{H}), 2.97(\mathrm{~d}, J=3.9 \mathrm{~Hz}, 3 \mathrm{H}), 2.67$ $-2.51(\mathrm{~m}, 4 \mathrm{H}), 2.33-2.20(\mathrm{~m}, 1 \mathrm{H}), 2.15(\mathrm{~s}, 3 \mathrm{H}), 2.11-2.07(\mathrm{~m}, 1 \mathrm{H}), 2.04(\mathrm{~s}, 3 \mathrm{H}), 1.87$ $-1.74(\mathrm{~m}, 6 \mathrm{H}), 1.01(\mathrm{~d}, J=5.6 \mathrm{~Hz}, 12 \mathrm{H}) .{ }^{13} \mathrm{C} \mathrm{NMR}\left(101 \mathrm{MHz}, \mathrm{CDCl}_{3}\right) \delta 171.9,170.2$, $169.1,166.3,166.1,153.7,153.1,133.3,132.0,125.7,124.7,123.8,122.6,121.0,120.3$, $112.9,112.7,77.4,77.2,68.1,53.9,38.1,37.9,36.6,31.7,30.7,26.9,25.6,24.5,22.8,22.7$, 22.7, 15.5. HRMS(ESI): calcd for $\mathrm{C}_{35} \mathrm{H}_{51} \mathrm{~N}_{5} \mathrm{O}_{7} \mathrm{~S}: 686.3582\left(\mathrm{M}+\mathrm{H}^{+}\right)$, found: $686.3586(\mathrm{M}$ $\left.+\mathrm{H}^{+}\right)$.

Compound 2f. Purification by flash column chromatography (petroleum ether/acetone = 2/1) to afford a white solid (462 mg, 66\%). ${ }^{1} \mathrm{H}$ NMR (400 MHz, $\left.\mathrm{CDCl}_{3}\right) \delta 9.75(\mathrm{~s}, 1 \mathrm{H})$, $9.56(\mathrm{~s}, 1 \mathrm{H}), 8.44(\mathrm{~d}, J=7.1 \mathrm{~Hz}, 1 \mathrm{H}), 8.41-8.36(\mathrm{~m}, 1 \mathrm{H}), 8.27-8.25(\mathrm{~m}, 1 \mathrm{H}), 8.16(\mathrm{~d}, J$ $=4.4 \mathrm{~Hz}, 1 \mathrm{H}), 8.14-8.10(\mathrm{~m}, 1 \mathrm{H}), 8.07-8.01(\mathrm{~m}, 1 \mathrm{H}), 6.94(\mathrm{~d}, J=9.0 \mathrm{~Hz}, 1 \mathrm{H}), 6.87(\mathrm{~d}$, $J=8.8 \mathrm{~Hz}, 1 \mathrm{H}), 6.55(\mathrm{~s}, 1 \mathrm{H}), 4.68-4.53(\mathrm{~m}, 1 \mathrm{H}), 4.25(\mathrm{dd}, J=17.7,6.5 \mathrm{~Hz}, 1 \mathrm{H}), 4.17-$ $3.99(\mathrm{~m}, 5 \mathrm{H}), 3.01(\mathrm{~d}, J=4.2 \mathrm{~Hz}, 3 \mathrm{H}), 2.63-2.53(\mathrm{~m}, 1 \mathrm{H}), 2.42(\mathrm{~m}, 1 \mathrm{H}), 2.22(\mathrm{~s}, 3 \mathrm{H})$, $1.93-1.75(\mathrm{~m}, 6 \mathrm{H}), 1.36(\mathrm{~d}, J=6.5 \mathrm{~Hz}, 3 \mathrm{H}), 1.01(\mathrm{~d}, J=5.9 \mathrm{~Hz}, 12 \mathrm{H}) .{ }^{13} \mathrm{C} \mathrm{NMR}(151$ MHz, DMSO- $\left.d_{6}\right) \delta 170.6,167.9,167.4,165.1,163.4,152.1,152.1,132.5,132.0,123.1$, $122.9,122.9,122.5,121.5,113.4,113.2,67.4,67.2,42.6,42.4,41.2,37.2,37.1,26.1,24.8$, 24.5, 23.8, 22.4, 22.4, 22.2, 19.8. HRMS(ESI): calcd for $\mathrm{C}_{33} \mathrm{H}_{47} \mathrm{~N}_{5} \mathrm{O}_{7}: 626.3548\left(\mathrm{M}+\mathrm{H}^{+}\right)$, found: $626.3551\left(\mathrm{M}+\mathrm{H}^{+}\right)$.

Compound 2g. Purification by flash column chromatography (petroleum ether/acetone = $2 / 1)$ to afford a white solid (519 mg, 66\%). ${ }^{1} \mathrm{H}$ NMR $\left(400 \mathrm{MHz}, \mathrm{CDCl}_{3}\right) \delta 9.71(\mathrm{~s}, 1 \mathrm{H})$, $9.43(\mathrm{~s}, 1 \mathrm{H}), 8.50(\mathrm{~d}, J=8.4 \mathrm{~Hz}, 1 \mathrm{H}), 8.41-8.34(\mathrm{~m}, 1 \mathrm{H}), 8.26-8.22(\mathrm{~m}, 1 \mathrm{H}), 8.19-8.15$ $(\mathrm{m}, 1 \mathrm{H}), 8.13-8.10(\mathrm{~m}, 1 \mathrm{H}), 8.04(\mathrm{dd}, J=9.0,2.7 \mathrm{~Hz}, 1 \mathrm{H}), 7.34-7.28(\mathrm{~m}, 3 \mathrm{H}), 7.23-$ $7.19(\mathrm{~m}, 2 \mathrm{H}), 6.93(\mathrm{~d}, J=9.1 \mathrm{~Hz}, 1 \mathrm{H}), 6.83(\mathrm{~d}, J=9.0 \mathrm{~Hz}, 1 \mathrm{H}), 6.40(\mathrm{t}, J=5.5 \mathrm{~Hz}, 1 \mathrm{H})$, $4.90-4.78(\mathrm{~m}, 1 \mathrm{H}), 4.30(\mathrm{dd}, J=17.4,7.3 \mathrm{~Hz}, 1 \mathrm{H}), 4.19-4.11(\mathrm{~m}, 2 \mathrm{H}), 4.04$ (q, $J=5.9$ $\mathrm{Hz}, 2 \mathrm{H}), 3.93$ (dd, $J=17.3,4.5 \mathrm{~Hz}, 1 \mathrm{H}), 3.06-2.89(\mathrm{~m}, 4 \mathrm{H}), 2.93-2.86(\mathrm{~m}, 1 \mathrm{H}), 2.65$ (dd, $J=13.7,2.6 \mathrm{~Hz}, 1 \mathrm{H}), 2.39$ (dd, $J=13.7,10.6 \mathrm{~Hz}, 1 \mathrm{H}), 2.19$ (s, 3H), $1.90-1.73$ (m, $4 \mathrm{H}), 1.63(\mathrm{q}, J=6.8 \mathrm{~Hz}, 2 \mathrm{H}), 1.09-0.92(\mathrm{~m}, 12 \mathrm{H}) .{ }^{13} \mathrm{C} \mathrm{NMR}\left(101 \mathrm{MHz}, \mathrm{CDCl}_{3}\right) \delta 171.5$, 169.2 , 167.9, 166.4, 166.2, 153.7, 152.8, 137.1, 133.5, 129.4, 128.9, 127.2, 124.8, 124.5, $122.7,121.1,120.7,112.9,112.7,77.4,68.1,68.0,49.3,44.5,43.8,42.2,38.1,37.9,26.8$, 25.6, 25.3, 24.6, 22.7. HRMS(ESI): calcd for $\mathrm{C}_{39} \mathrm{H}_{51} \mathrm{~N}_{5} \mathrm{O}_{7}: 702.3861\left(\mathrm{M}+\mathrm{H}^{+}\right)$, found: $702.3871\left(\mathrm{M}+\mathrm{H}^{+}\right)$.

Compound $\mathbf{2 h}$. The crude product was washed with methanol and a white solid was obtained by recrystallization (dichloromethane/ethyl acetate/methanol $=20: 10: 1)(630 \mathrm{mg}$, 
86\%). ${ }^{1} \mathrm{H}$ NMR $\left(400 \mathrm{MHz}, \mathrm{CDCl}_{3}\right) \delta 9.82(\mathrm{~s}, 1 \mathrm{H}), 9.48(\mathrm{~s}, 1 \mathrm{H}), 8.44(\mathrm{t}, J=12.0 \mathrm{~Hz}, 2 \mathrm{H})$, $8.31(\mathrm{~d}, J=2.4 \mathrm{~Hz}, 1 \mathrm{H}), 8.24-8.22(\mathrm{~m}, 1 \mathrm{H}), 8.19(\mathrm{q}, J=4.0 \mathrm{~Hz}, 1 \mathrm{H}), 8.09-8.04(\mathrm{~m}, 1 \mathrm{H})$, $6.96(\mathrm{~d}, J=9.4 \mathrm{~Hz}, 1 \mathrm{H}), 6.93(\mathrm{~d}, J=9.4 \mathrm{~Hz}, 1 \mathrm{H}), 6.43-6.37(\mathrm{~m}, 1 \mathrm{H}), 4.42-4.34(\mathrm{~m}, 1 \mathrm{H})$, $4.31-3.99(\mathrm{~m}, 6 \mathrm{H}), 3.02(\mathrm{~d}, J=4.4 \mathrm{~Hz}, 3 \mathrm{H}), 2.63(\mathrm{dd}, J=14.4,1.3 \mathrm{~Hz}, 1 \mathrm{H}), 2.34(\mathrm{dd}, J$ $=13.7,10.3 \mathrm{~Hz}, 1 \mathrm{H}), 2.23(\mathrm{~s}, 3 \mathrm{H}), 1.91-1.70(\mathrm{~m}, 7 \mathrm{H}), 1.04-0.98(\mathrm{~m}, 18 \mathrm{H}) .{ }^{13} \mathrm{C} \mathrm{NMR}$ (101 MHz, DMSO) $\delta 170.8,167.9,167.4,165.1,163.9,152.1,132.5,132.0,123.1,123.0$, $122.9,122.6,121.7,121.5,113.4,113.1,67.4,67.2,51.5,42.5,37.6,37.3,37.2,30.8,26.2$, 24.8, 24.4, 23.8, 22.4, 22.4, 22.2, 19.4, 18.1. HRMS(ESI): calcd for $\mathrm{C}_{35} \mathrm{H}_{51} \mathrm{~N}_{5} \mathrm{O}_{7}: 654.3861$ $\left(\mathrm{M}+\mathrm{H}^{+}\right)$, found: $654.3864\left(\mathrm{M}+\mathrm{H}^{+}\right)$.

Compound 2i. Purification by flash column chromatography (petroleum ether/acetone $=$ $2 / 1)$ to afford a white solid (505 mg, 63\%). ${ }^{1} \mathrm{H}$ NMR $\left(600 \mathrm{MHz}\right.$, DMSO- $\left.d_{6}\right) \delta 10.08(\mathrm{~s}, 1 \mathrm{H})$, 9.89 (s, $1 \mathrm{H}), 8.35(\mathrm{~d}, J=8.1 \mathrm{~Hz}, 1 \mathrm{H}), 8.25(\mathrm{~d}, J=7.9 \mathrm{~Hz}, 1 \mathrm{H}), 8.04$ (t, $J=5.4 \mathrm{~Hz}, 1 \mathrm{H})$, $7.91(\mathrm{dd}, J=9.9,2.7 \mathrm{~Hz}, 2 \mathrm{H}), 7.74(\mathrm{dd}, J=8.9,2.8 \mathrm{~Hz}, 1 \mathrm{H}), 7.70(\mathrm{dd}, J=8.9,2.8 \mathrm{~Hz}, 1 \mathrm{H})$, 7.28 (d, $J=7.5 \mathrm{~Hz}, 2 \mathrm{H}), 7.23$ (t, $J=7.6 \mathrm{~Hz}, 2 \mathrm{H}), 7.15(\mathrm{t}, J=7.2 \mathrm{~Hz}, 1 \mathrm{H}), 7.10$ (d, $J=9.0$ $\mathrm{Hz}, 1 \mathrm{H}), 7.06(\mathrm{~d}, J=9.0 \mathrm{~Hz}, 1 \mathrm{H}), 4.73-4.60(\mathrm{~m}, 1 \mathrm{H}), 4.23(\mathrm{dt}, J=14.3,7.2 \mathrm{~Hz}, 1 \mathrm{H}), 4.10$ (t, $J=6.6 \mathrm{~Hz}, 2 \mathrm{H}), 4.06$ (t, $J=6.4 \mathrm{~Hz}, 2 \mathrm{H}), 3.04$ (dd, $J=13.8,4.8 \mathrm{~Hz}, 1 \mathrm{H}), 2.85$ (dd, $J=$ 13.6, $9.8 \mathrm{~Hz}, 1 \mathrm{H}), 2.80(\mathrm{~d}, J=4.7 \mathrm{~Hz}, 3 \mathrm{H}), 2.45(\mathrm{dd}, J=14.2,4.7 \mathrm{~Hz}, 1 \mathrm{H}), 2.23(\mathrm{dd}, J=$ 14.1, $7.9 \mathrm{~Hz}, 1 \mathrm{H}), 2.00(\mathrm{~s}, 3 \mathrm{H}), 1.79-1.57(\mathrm{~m}, 6 \mathrm{H}), 0.97$ (d, $J=6.6 \mathrm{~Hz}, 3 \mathrm{H}), 0.94$ (d, $J=$ $6.5 \mathrm{~Hz}, 6 \mathrm{H}), 0.91-0.86(\mathrm{~m}, 6 \mathrm{H}) .{ }^{13} \mathrm{C} \mathrm{NMR}\left(151 \mathrm{MHz}, \mathrm{CDCl}_{3}\right) \delta 170.9,169.7,169.08$, $166.32,165.61,153.48,152.83,137.16,133.29,132.18,129.80,128.42,126.75,124.5$, $124.2,122.9,121.1,120.8,112.9,112.5,68.1,67.8,54.7,45.9,43.9,38.1,38.1,26.8,25.6$, 25.3, 24.4, 22.8, 22.7, 22.7, 22.5, 21.8. HRMS(ESI): calcd for $\mathrm{C}_{40} \mathrm{H}_{53} \mathrm{~N}_{5} \mathrm{O}_{7}: 716.4018$ (M $\left.+\mathrm{H}^{+}\right)$, found: $716.4014\left(\mathrm{M}+\mathrm{H}^{+}\right)$.

Compound 2j. Purification by flash column chromatography (petroleum ether/acetone $=$ $2 / 1)$ to afford a white solid (449 mg, 56\%). ${ }^{1} \mathrm{H}$ NMR $\left(600 \mathrm{MHz}\right.$, DMSO- $\left.d_{6}\right) \delta 10.07(\mathrm{~s}, 1 \mathrm{H})$, $9.89(\mathrm{~s}, 1 \mathrm{H}), 8.36(\mathrm{~d}, J=8.3 \mathrm{~Hz}, 1 \mathrm{H}), 8.31(\mathrm{~d}, J=8.1 \mathrm{~Hz}, 1 \mathrm{H}), 8.05(\mathrm{q}, J=4.8 \mathrm{~Hz}, 1 \mathrm{H})$, $7.91(\mathrm{~d}, J=2.9 \mathrm{~Hz}, 1 \mathrm{H}), 7.90(\mathrm{~d}, J=2.8 \mathrm{~Hz}, 1 \mathrm{H}), 7.74$ (dd, $J=8.9,2.8 \mathrm{~Hz}, 1 \mathrm{H}), 7.69$ (dd, $J=8.9,2.9 \mathrm{~Hz}, 1 \mathrm{H}), 7.28(\mathrm{~d}, J=7.2 \mathrm{~Hz}, 2 \mathrm{H}), 7.23(\mathrm{t}, J=7.6 \mathrm{~Hz}, 2 \mathrm{H}), 7.16(\mathrm{t}, J=7.3 \mathrm{~Hz}$, $1 \mathrm{H}), 7.10(\mathrm{~d}, J=9.0 \mathrm{~Hz}, 1 \mathrm{H}), 7.06(\mathrm{~d}, J=9.0 \mathrm{~Hz}, 1 \mathrm{H}), 4.67$ (dd, $J=14.0,8.9 \mathrm{~Hz}, 1 \mathrm{H})$, $4.24(\mathrm{dt}, J=13.8,6.9 \mathrm{~Hz}, 1 \mathrm{H}), 4.14-4.01(\mathrm{~m}, 4 \mathrm{H}), 3.04(\mathrm{dd}, J=13.8,5.2 \mathrm{~Hz}, 1 \mathrm{H}), 2.87$ $-2.82(\mathrm{~m}, 1 \mathrm{H}), 2.80(\mathrm{~d}, J=4.7 \mathrm{~Hz}, 3 \mathrm{H}), 2.41$ (dd, $J=14.2,4.9 \mathrm{~Hz}, 1 \mathrm{H}), 2.27$ (dd, $J=14.2$, $7.2 \mathrm{~Hz}, 1 \mathrm{H}), 2.00(\mathrm{~s}, 3 \mathrm{H}), 1.81-1.57(\mathrm{~m}, 6 \mathrm{H}), 0.99(\mathrm{~d}, J=6.6 \mathrm{~Hz}, 3 \mathrm{H}), 0.94(\mathrm{~d}, J=6.6$ $\mathrm{Hz}, 6 \mathrm{H}), 0.89$ (t, $J=6.7 \mathrm{~Hz}, 6 \mathrm{H}) .{ }^{13} \mathrm{C}$ NMR $\left(151 \mathrm{MHz}, \mathrm{CDCl}_{3}\right) \delta 171.7,170.0,169.4,166.3$, 165.7, 136.3, 133.5, 131.7, 129.1, 128.6, 127. 9, 127.1, 125.4, 125.2, 123.4, 121.1, 120.0, 113.0, 112.6, 77.2, 68.1, 67.8, 55.3, 46.5, 44.2, 38.2, 38.1, 37.9, 26.9, 25. 6, 25.4, 24.5, 22.9, 22.7, 22.7, 22.6. HRMS(ESI): calcd for $\mathrm{C}_{40} \mathrm{H}_{53} \mathrm{~N}_{5} \mathrm{O}_{7}: 716.4018\left(\mathrm{M}+\mathrm{H}^{+}\right)$, found: $716.4018\left(\mathrm{M}+\mathrm{H}^{+}\right)$. 


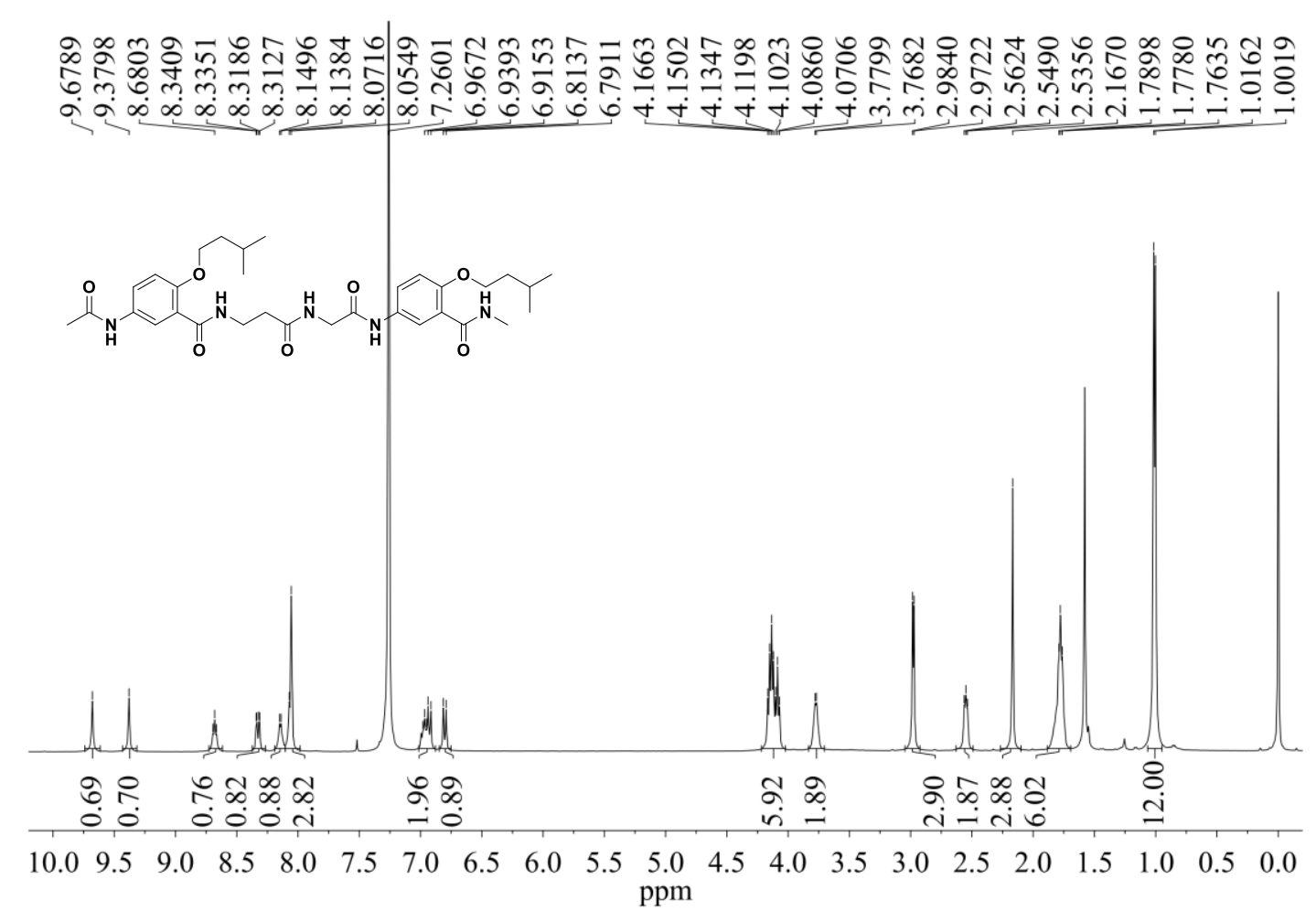

Figure S2. ${ }^{1} \mathrm{H} \mathrm{NMR}$ spectrum of compound $2 \mathbf{a}\left(\mathrm{CDCl}_{3}, 298 \mathrm{~K}, 400 \mathrm{MHz}\right)$

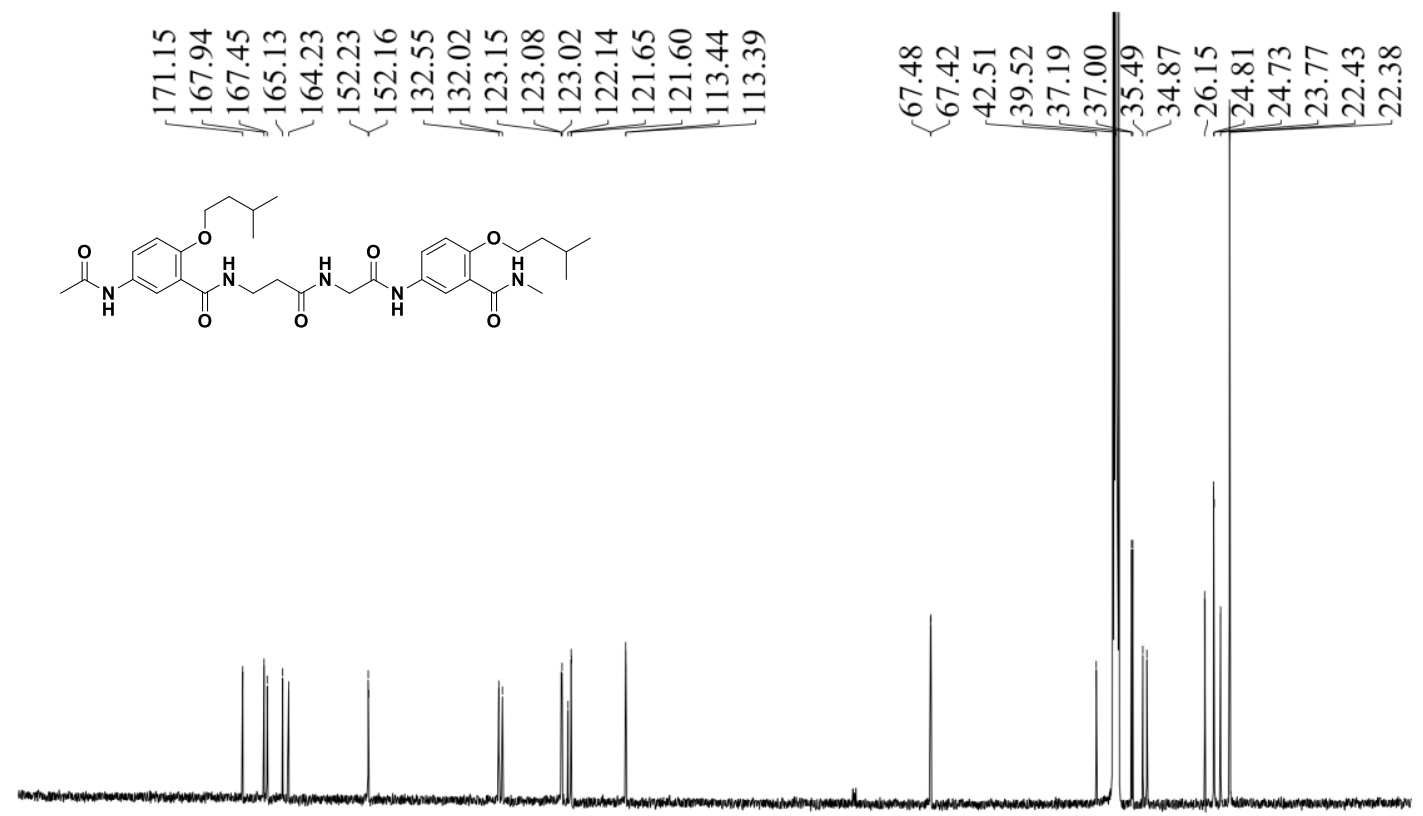

\begin{tabular}{lllllllllllllllllllllllll}
\hline 200 & 190 & 180 & 170 & 160 & 150 & 140 & 130 & 120 & 110 & 100 & 90 & 80 & 70 & 60 & 50 & 40 & 30 & 20 & 10 & 0 \\
$\mathrm{ppm}$
\end{tabular}

Figure S3. ${ }^{13} \mathrm{C}$ NMR spectrum of compound 2a (DMSO- $\left.d_{6}, 299 \mathrm{~K}, 151 \mathrm{MHz}\right)$ 


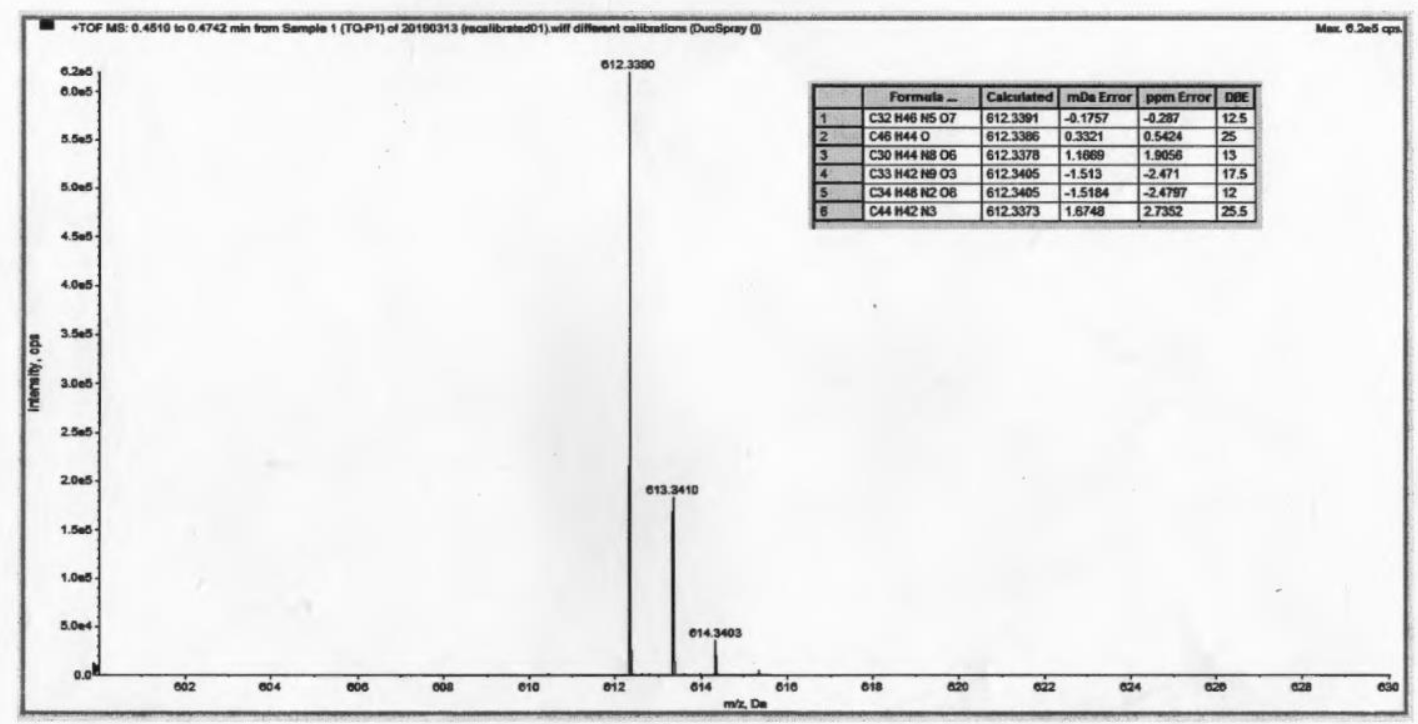

Figure S4. MS spectrum of compound 2a

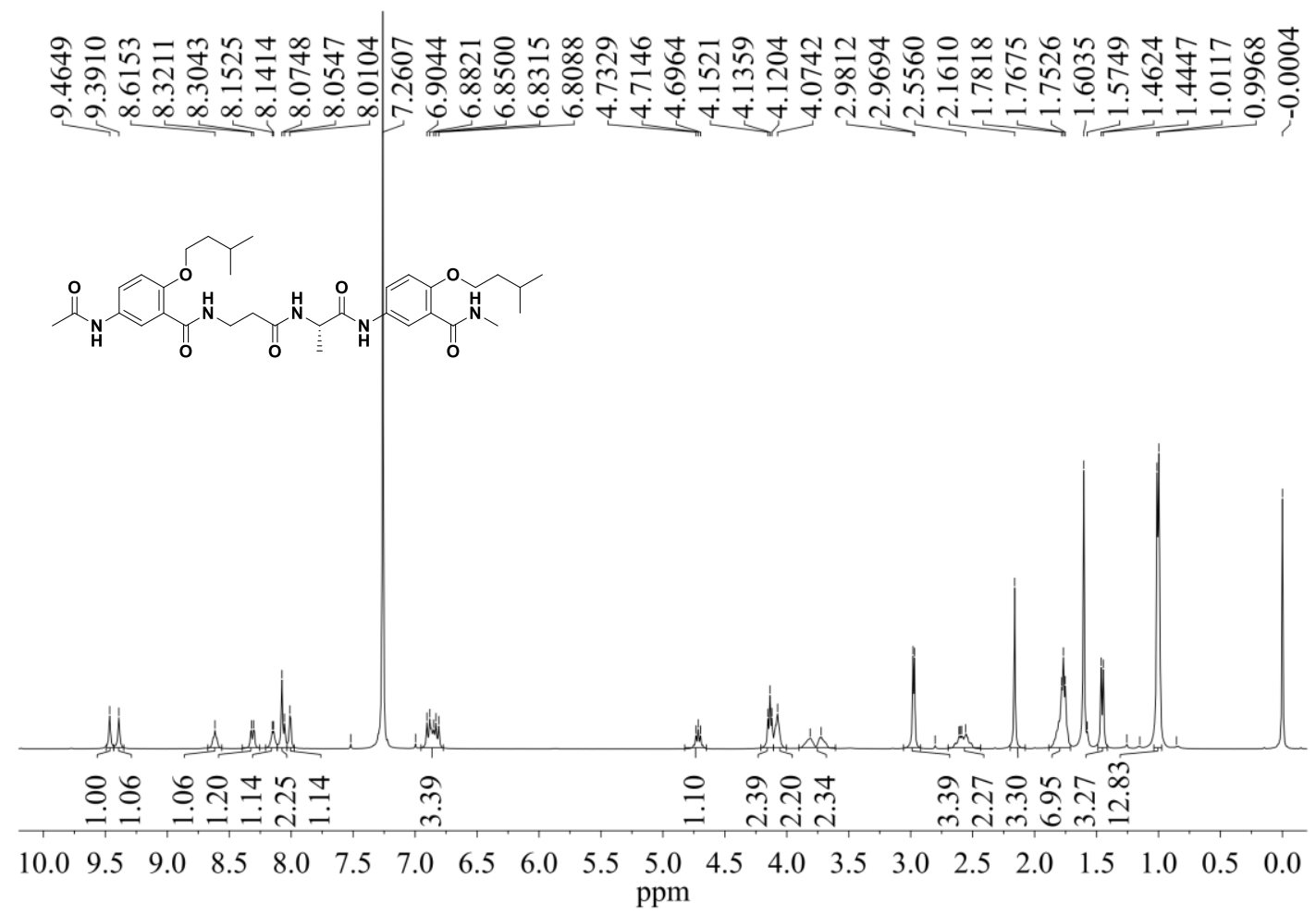

Figure S5. ${ }^{1} \mathrm{H}$ NMR spectrum of compound $\mathbf{2 b}\left(\mathrm{CDCl}_{3}, 298 \mathrm{~K}, 400 \mathrm{MHz}\right)$ 


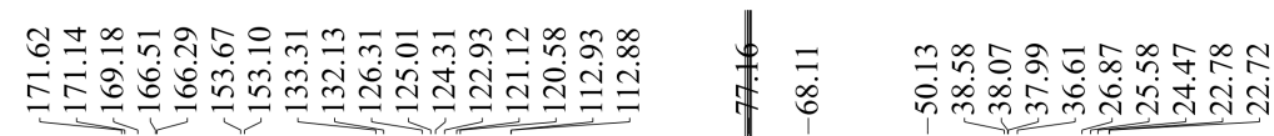<smiles>CC(=O)Nc1ccc(OCCC(C)C)c(C(=O)NCC(=O)NC(=O)Nc2ccc3c(c2)C(=O)N(C(C)C)OC3C(=O)NCC(C)C)c1</smiles>

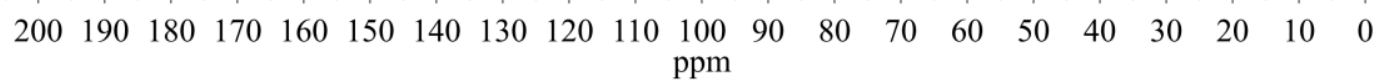

Figure S6. ${ }^{13} \mathrm{C}$ NMR spectrum of compound $\mathbf{2 b}\left(\mathrm{CDCl}_{3}, 296 \mathrm{~K}, 101 \mathrm{MHz}\right)$

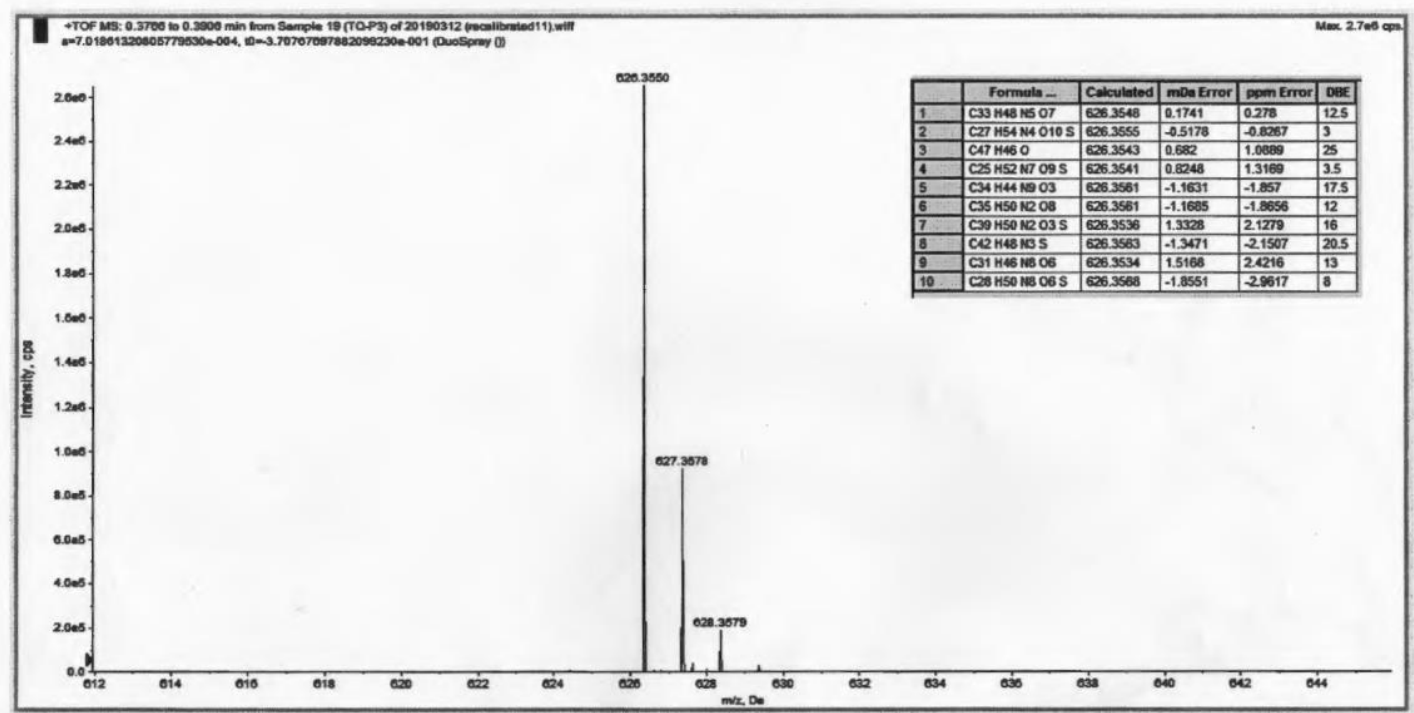

Figure S7. MS spectrum of compound $\mathbf{2 b}$. 


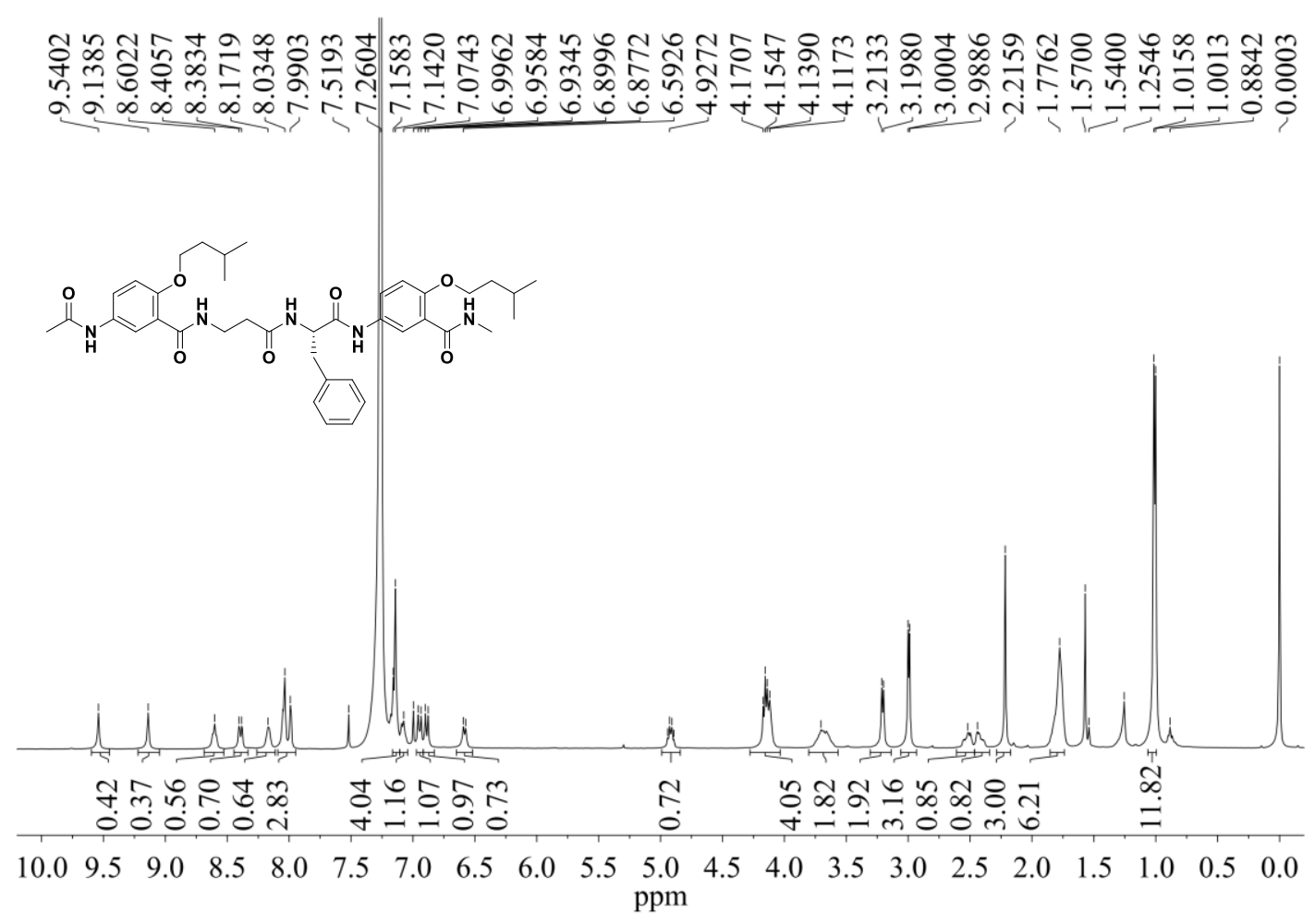

Figure S8. ${ }^{1} \mathrm{H} \mathrm{NMR}$ spectrum of compound $2 \mathbf{c}\left(\mathrm{CDCl}_{3}, 298 \mathrm{~K}, 400 \mathrm{MHz}\right)$

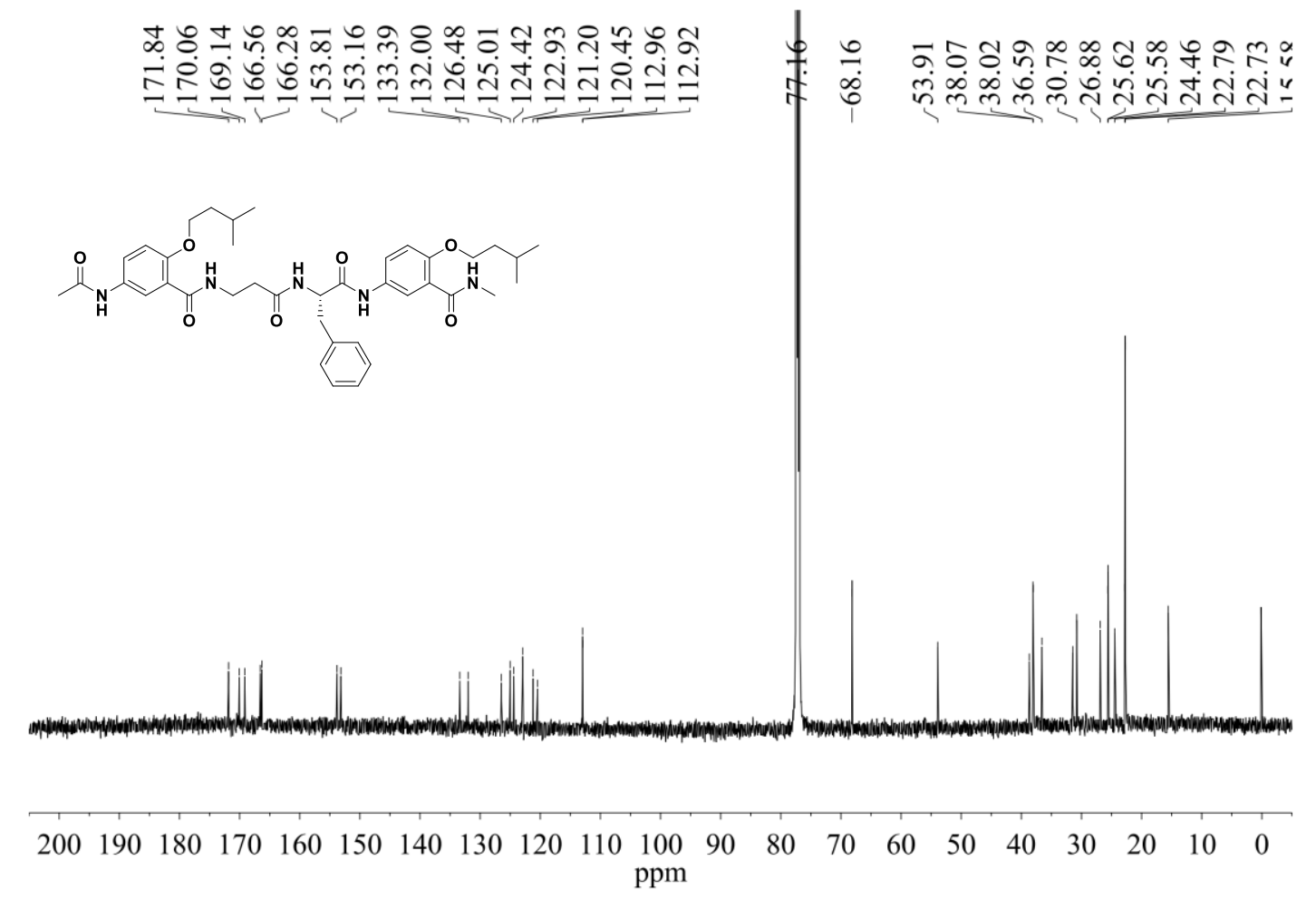

Figure S9. ${ }^{13} \mathrm{C} \mathrm{NMR}$ spectrum of compound $2 \mathrm{c}\left(\mathrm{CDCl}_{3}, 297 \mathrm{~K}, 151 \mathrm{MHz}\right)$ 


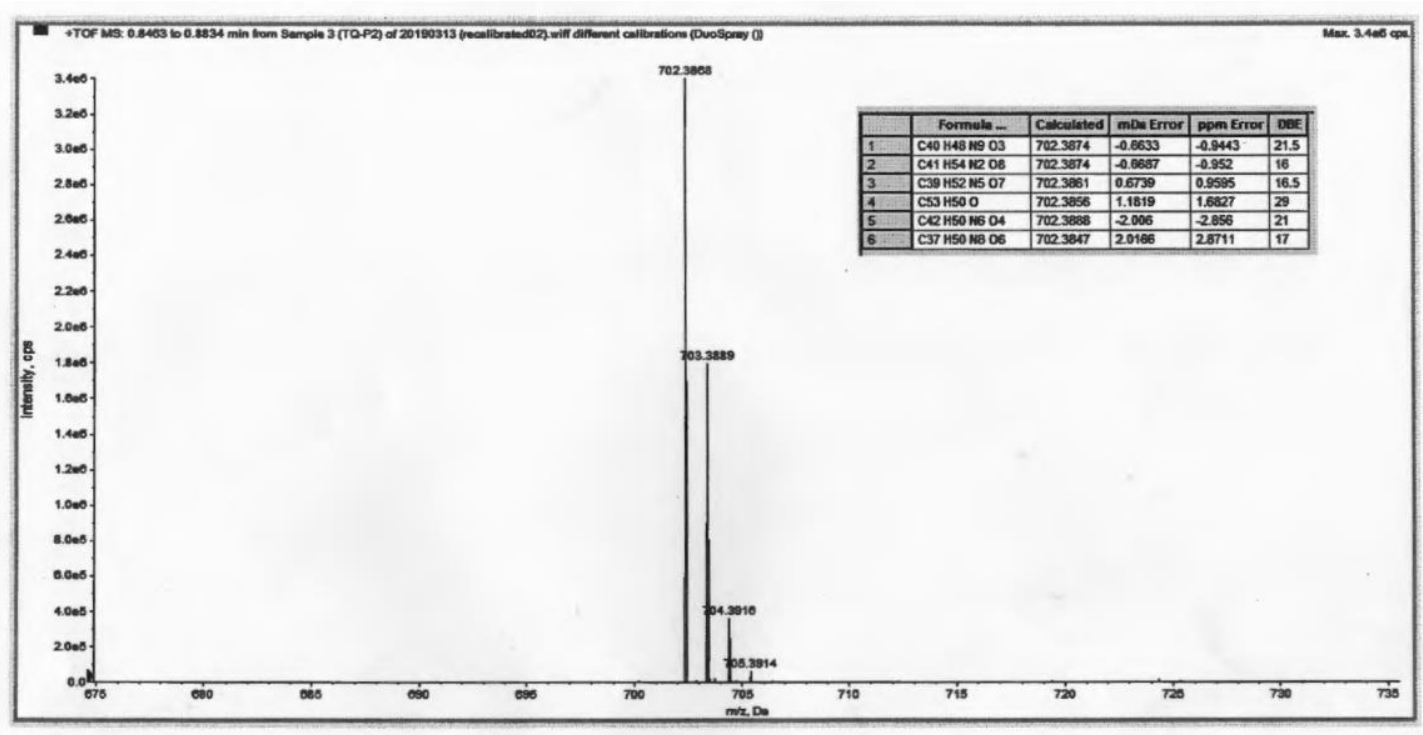

Figure S10. MS spectrum of compound 2c

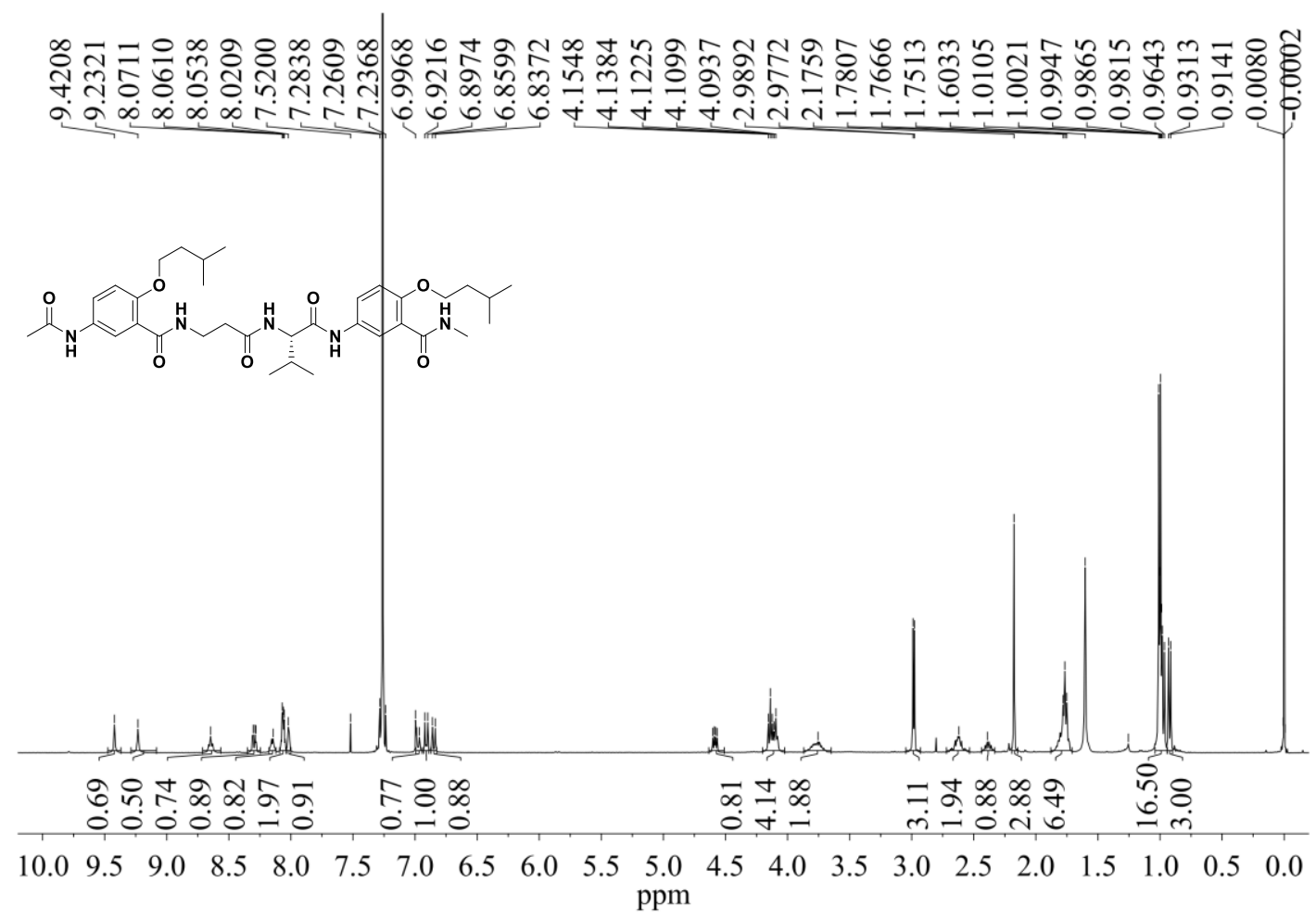

Figure S11. ${ }^{1} \mathrm{H} \mathrm{NMR}$ spectrum of compound $2 \mathbf{d}\left(\mathrm{CDCl}_{3}, 298 \mathrm{~K}, 400 \mathrm{MHz}\right)$ 


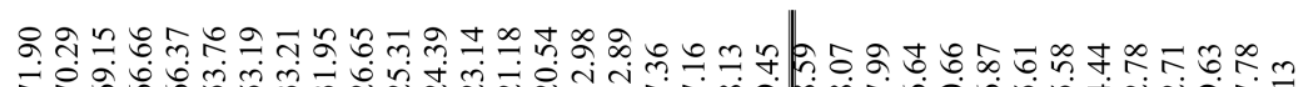

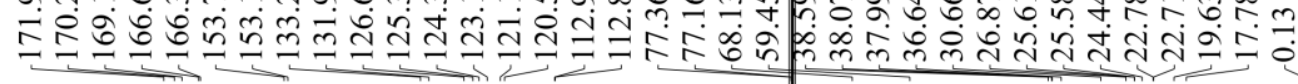

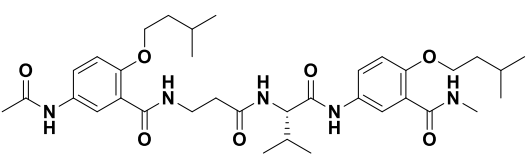

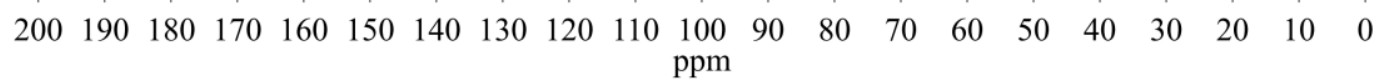

Figure S12. ${ }^{13} \mathrm{C}$ NMR spectrum of compound $2 \mathbf{d}\left(\mathrm{CDCl}_{3}, 298 \mathrm{~K}, 101 \mathrm{MHz}\right)$

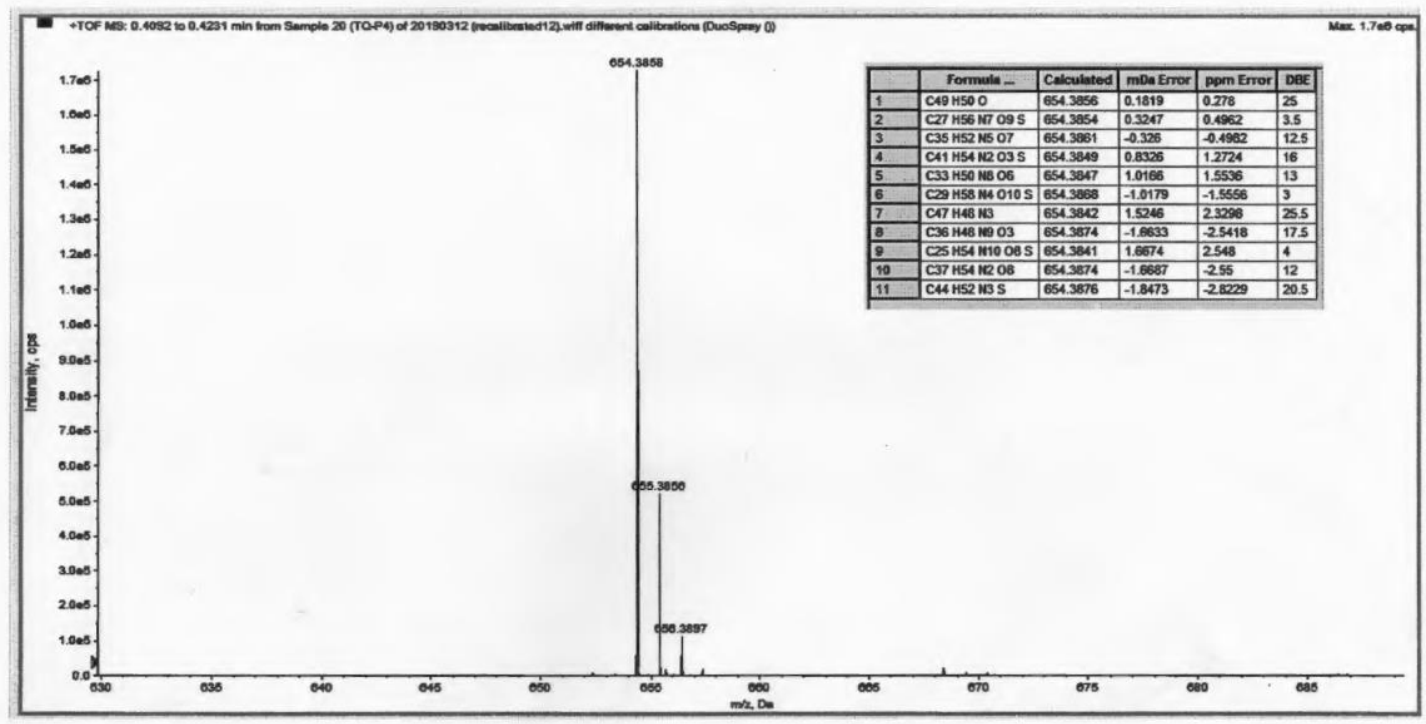

Figure S13. MS spectrum of compound 2d 


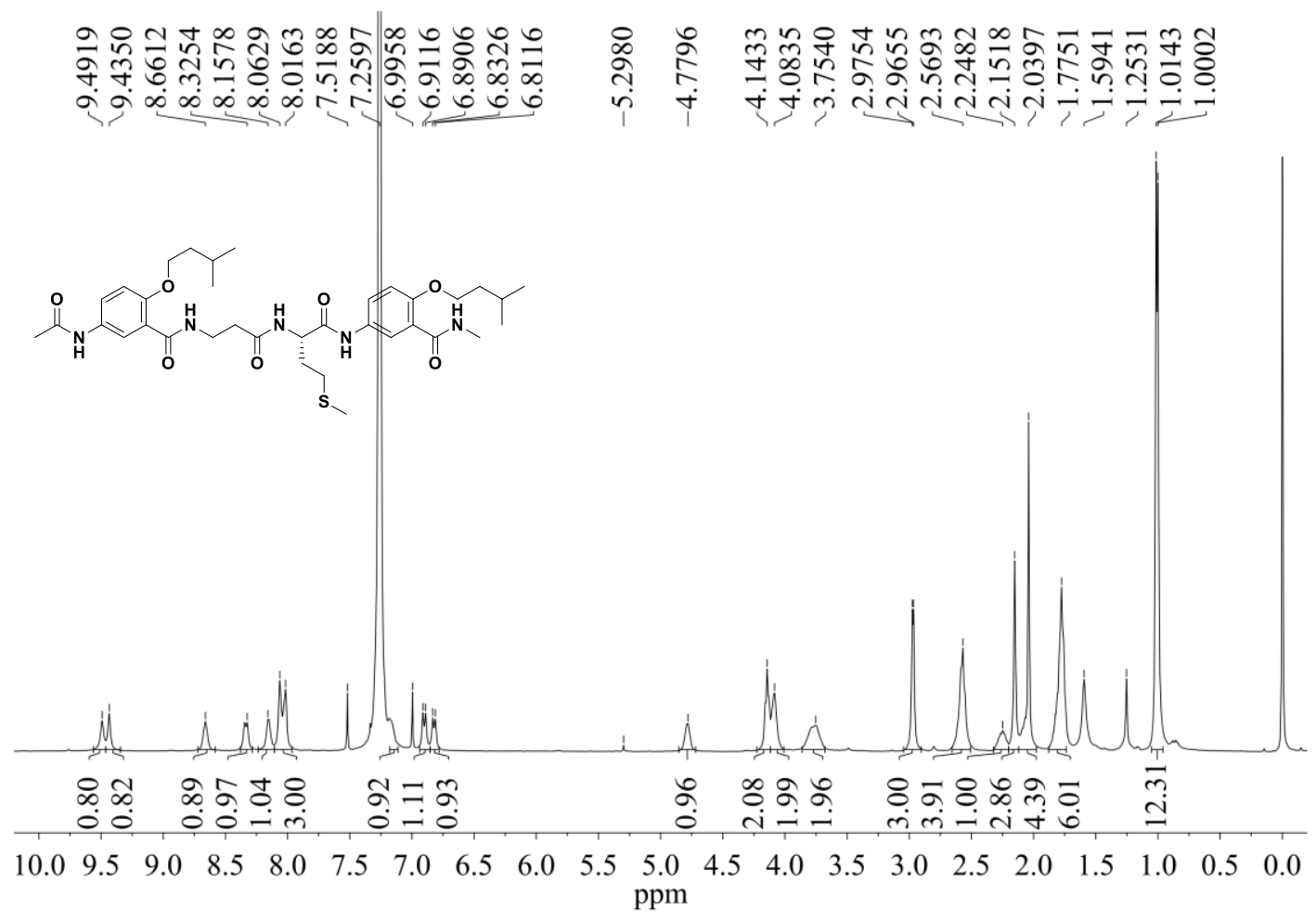

Figure S14. ${ }^{1} \mathrm{H}$ NMR spectrum of compound $2 \mathrm{e}\left(\mathrm{CDCl}_{3}, 298 \mathrm{~K}, 400 \mathrm{MHz}\right)$

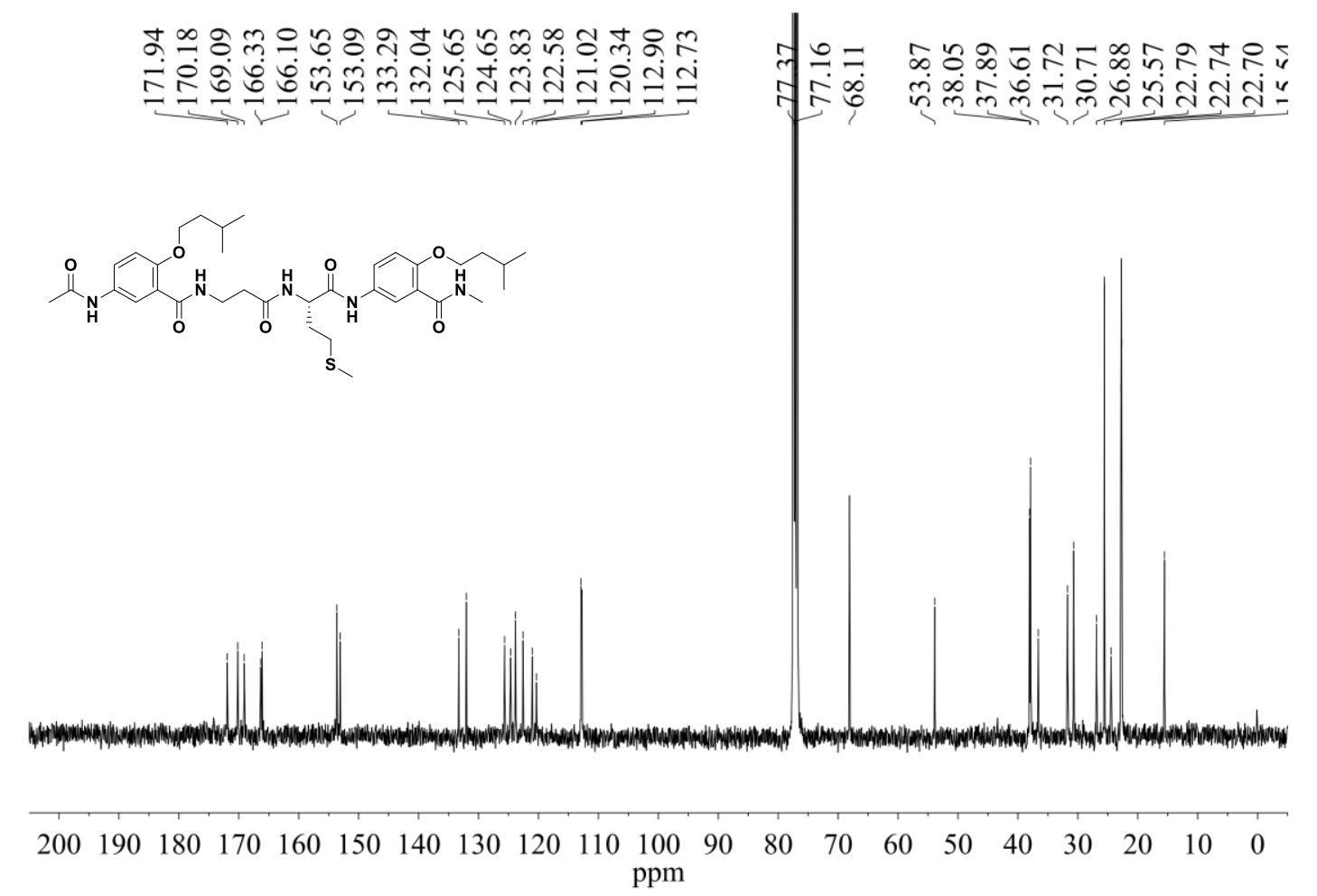

Figure S15. ${ }^{13} \mathrm{C}$ NMR spectrum of compound $2 \mathrm{e}\left(\mathrm{CDCl}_{3}, 296 \mathrm{~K}, 101 \mathrm{MHz}\right)$ 


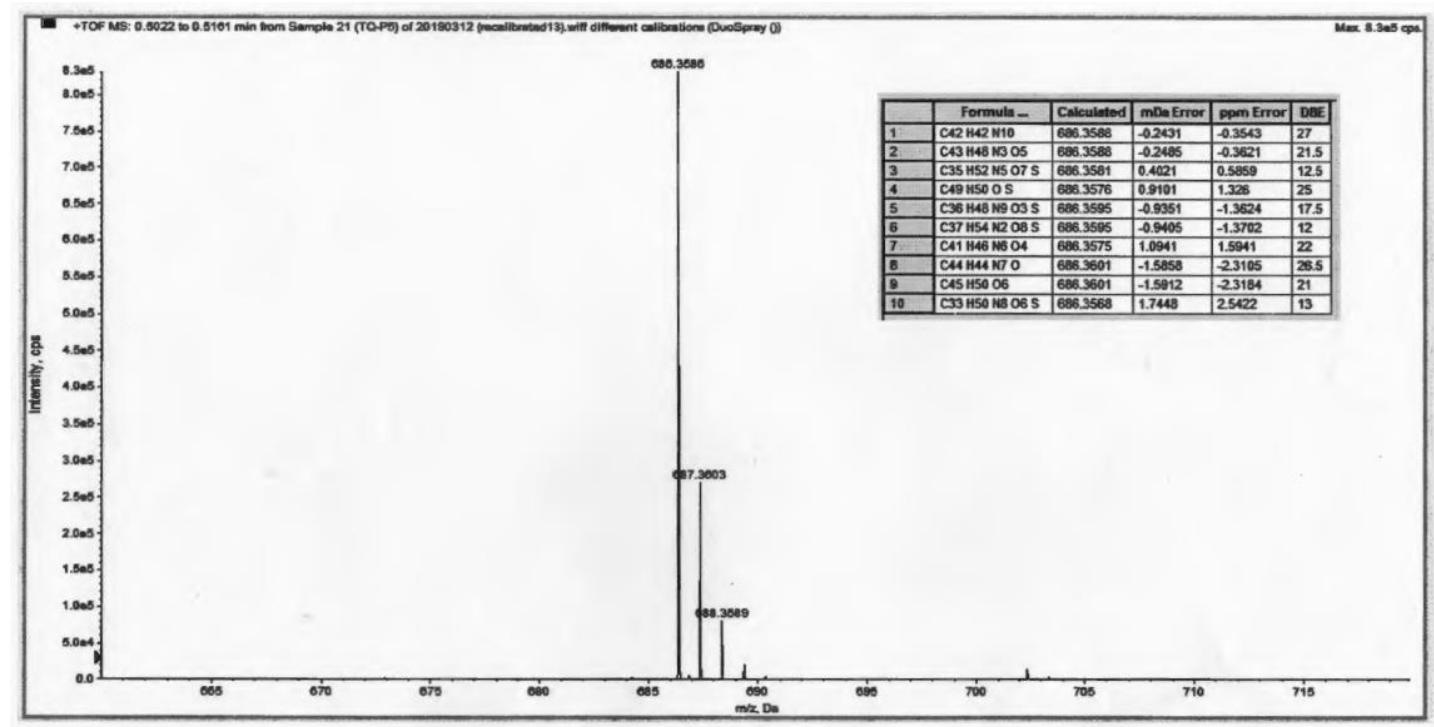

Figure S16. MS spectrum of compound 2e

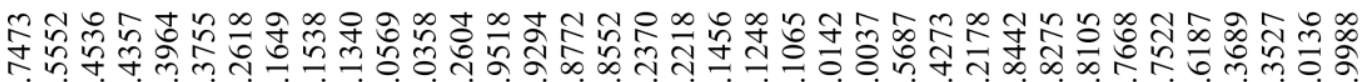
ốn

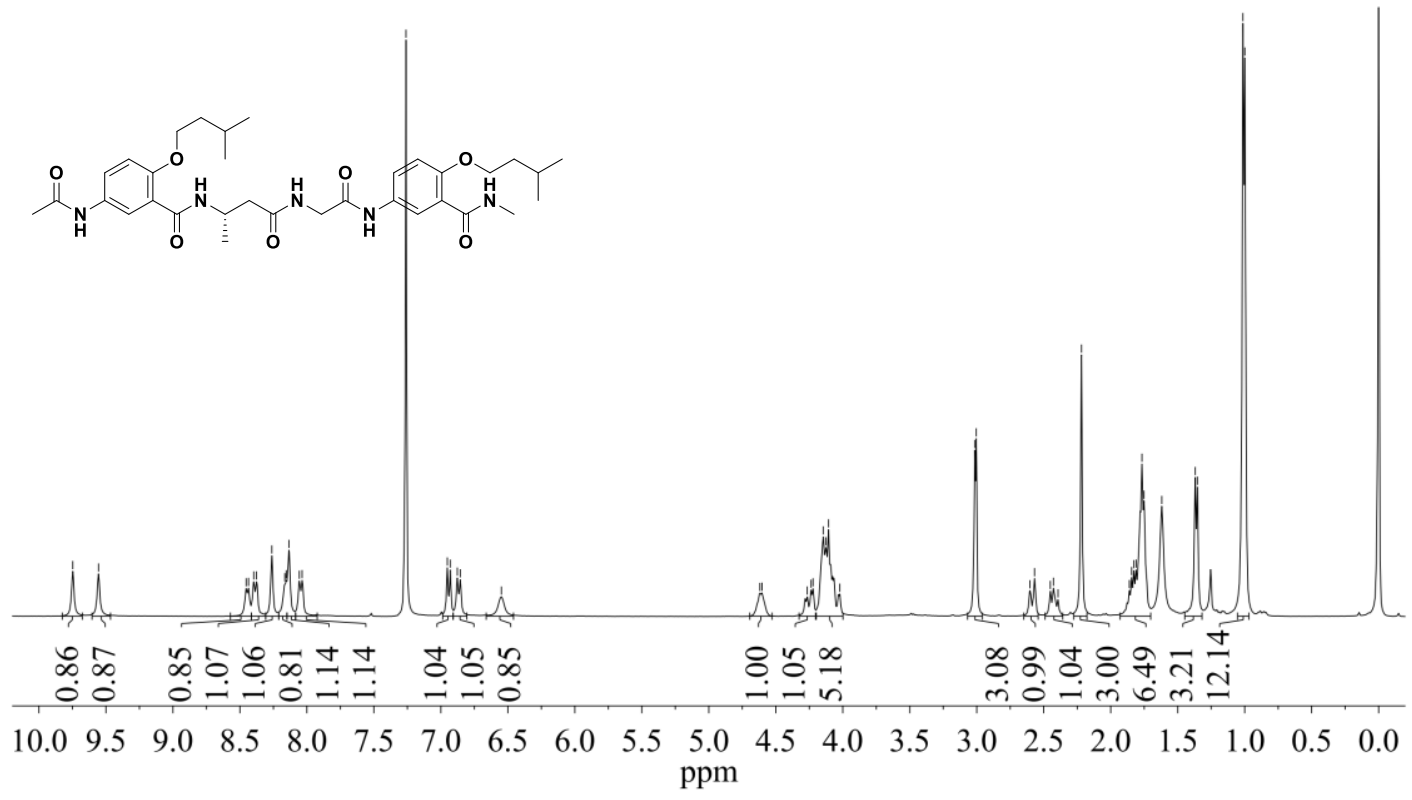

Figure S17. ${ }^{1} \mathrm{H}$ NMR spectrum of compound $2 \mathbf{f}\left(\mathrm{CDCl}_{3}, 298 \mathrm{~K}, 400 \mathrm{MHz}\right)$ 


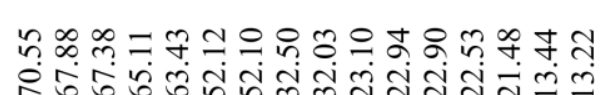

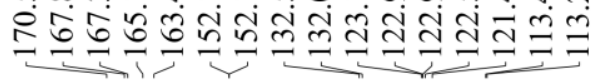

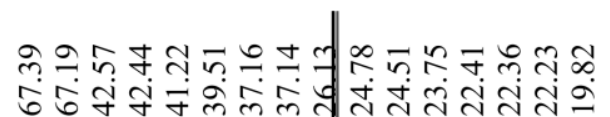

$\overbrace{}^{\frac{1}{2}}$

$\begin{array}{llllllllllllllllllllll}200 & 190 & 180 & 170 & 160 & 150 & 140 & 130 & 120 & 110 & 100 & 90 & 80 & 70 & 60 & 50 & 40 & 30 & 20 & 10 & 0\end{array}$

Figure S18. ${ }^{13} \mathrm{C}$ NMR spectrum of compound $2 \mathbf{f}\left(\mathrm{DMSO}-d_{6}, 298 \mathrm{~K}, 151 \mathrm{MHz}\right)$

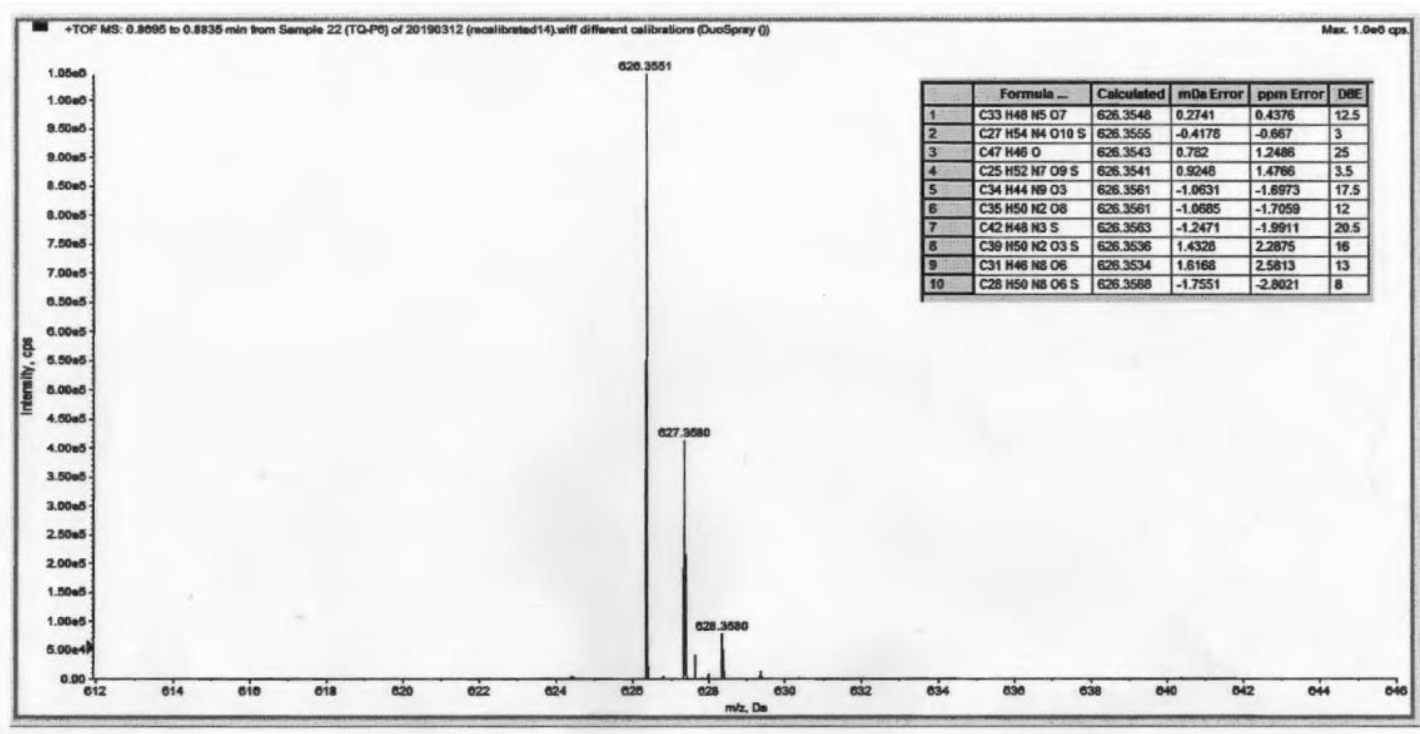

Figure S19. MS spectrum of compound $2 \mathbf{f}$ 


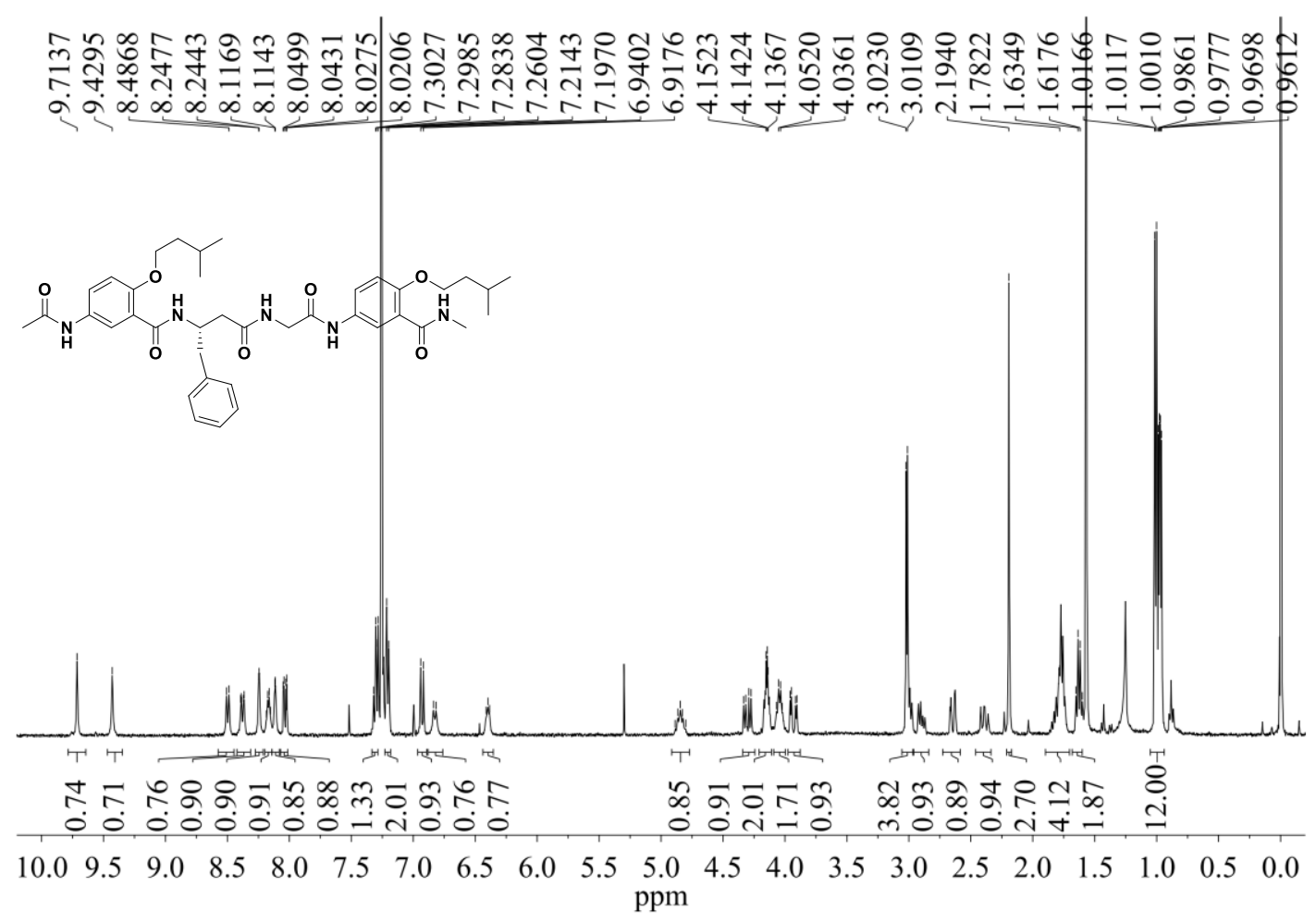

Figure S20. ${ }^{1} \mathrm{H} \mathrm{NMR}$ spectrum of compound $\mathbf{2 g}\left(\mathrm{CDCl}_{3}, 296 \mathrm{~K}, 400 \mathrm{MHz}\right)$

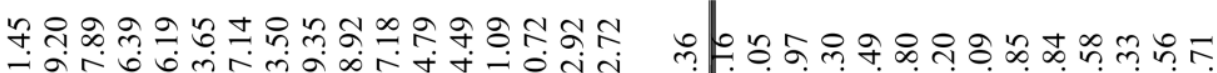
프은은
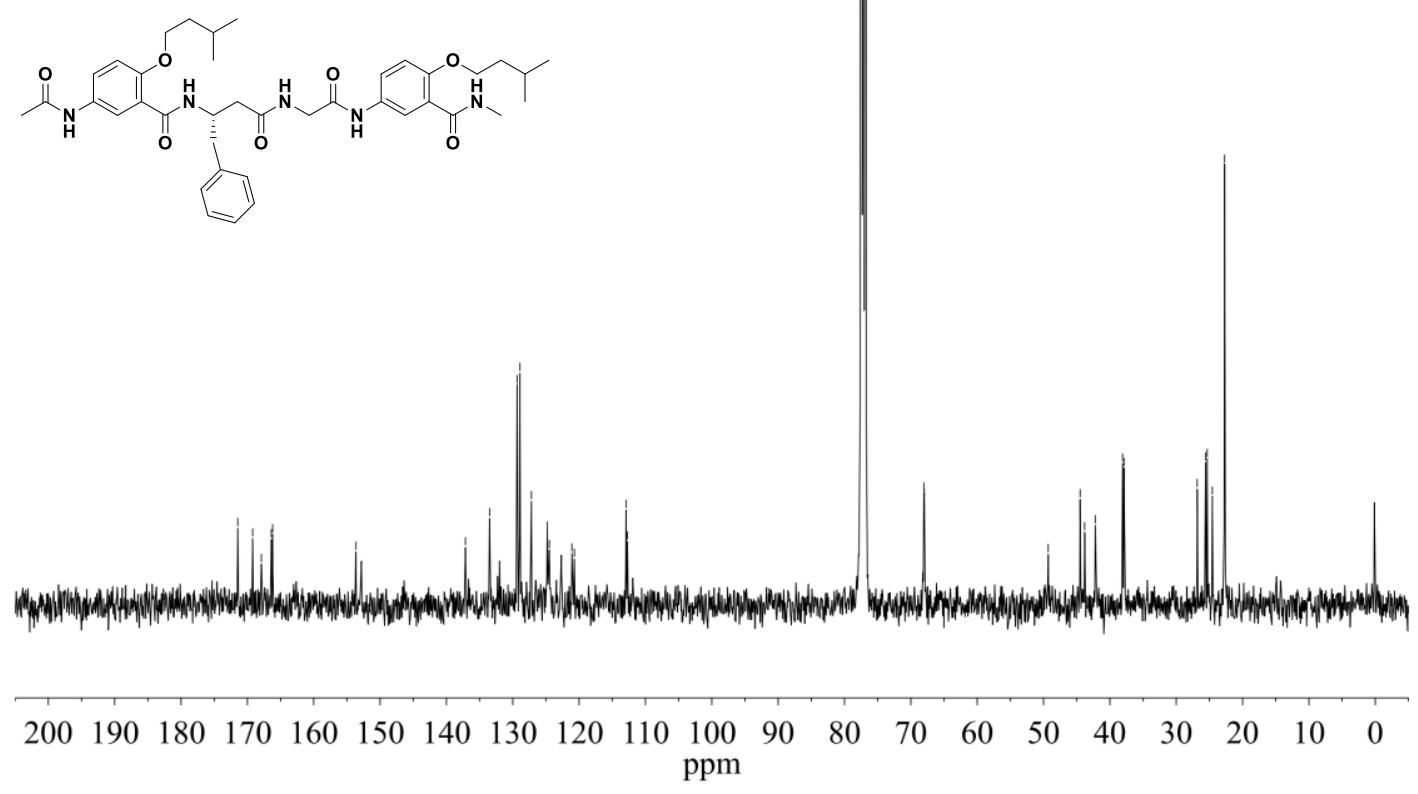

Figure S21. ${ }^{13} \mathrm{C}$ NMR spectrum of compound $\mathbf{2 g}\left(\mathrm{CDCl}_{3}, 295 \mathrm{~K}, 101 \mathrm{MHz}\right)$ 


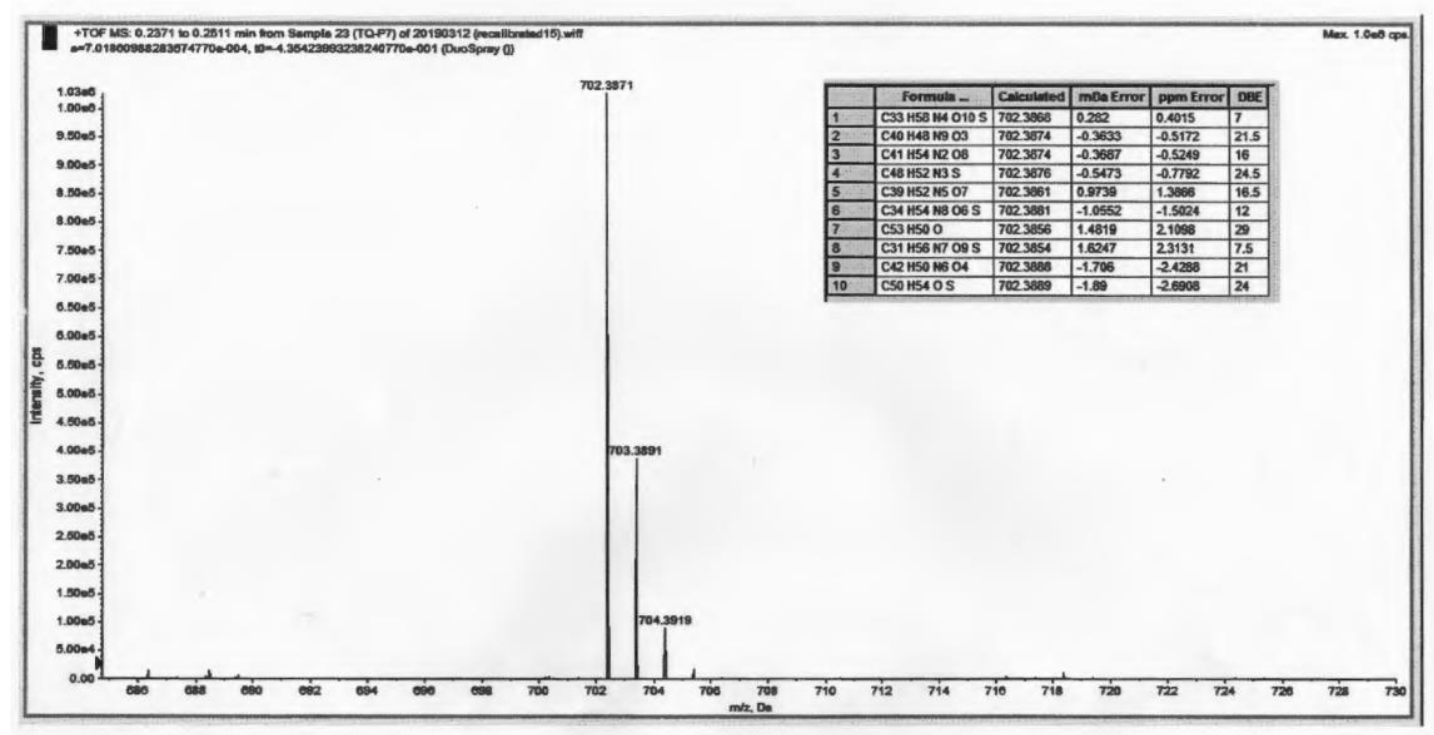

Figure S22. MS spectrum of compound $2 \mathbf{g}$

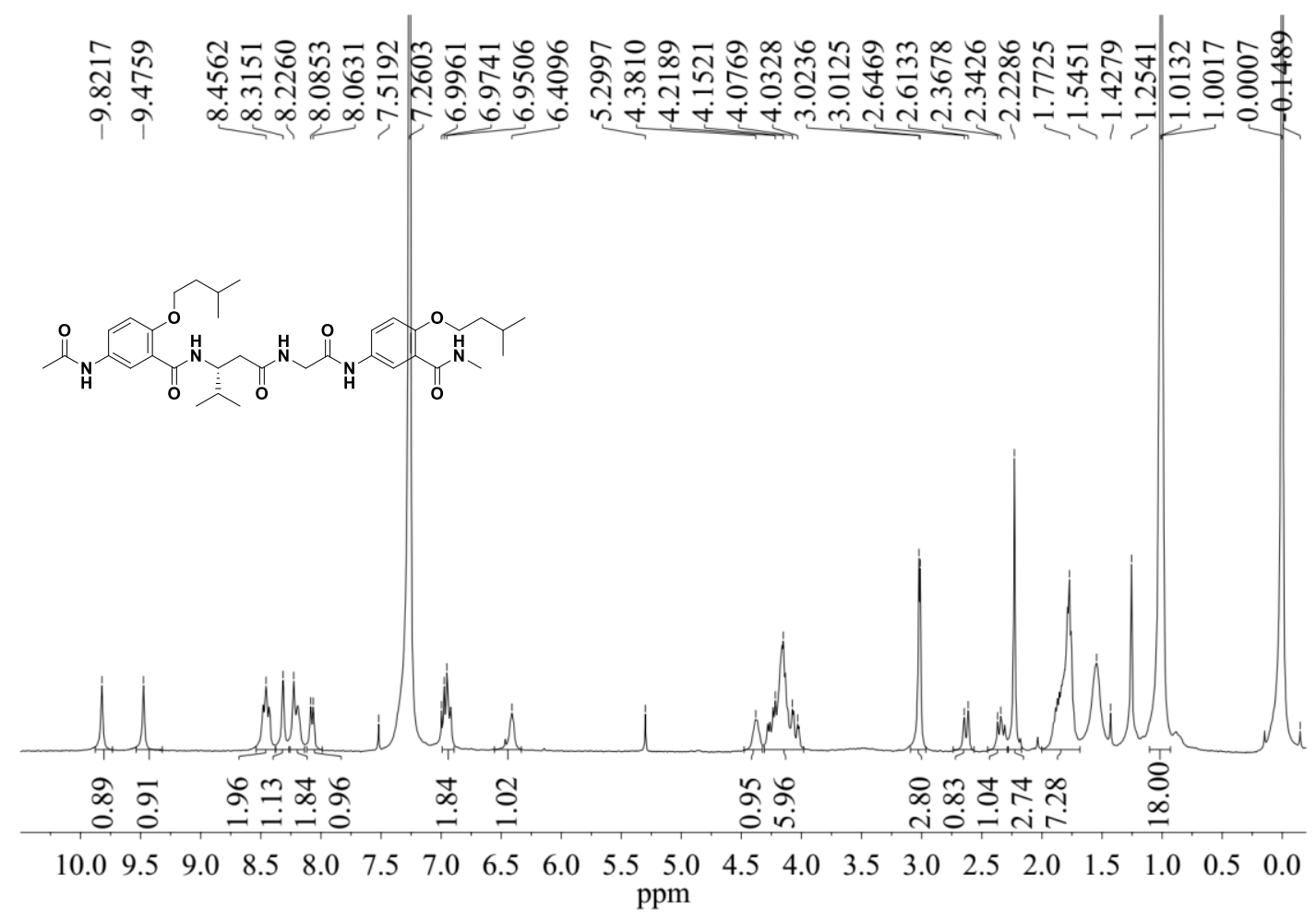

Figure S23. ${ }^{1} \mathrm{H} \mathrm{NMR}$ spectrum of compound $\mathbf{2 h}\left(\mathrm{CDCl}_{3}, 298 \mathrm{~K}, 400 \mathrm{MHz}\right)$ 


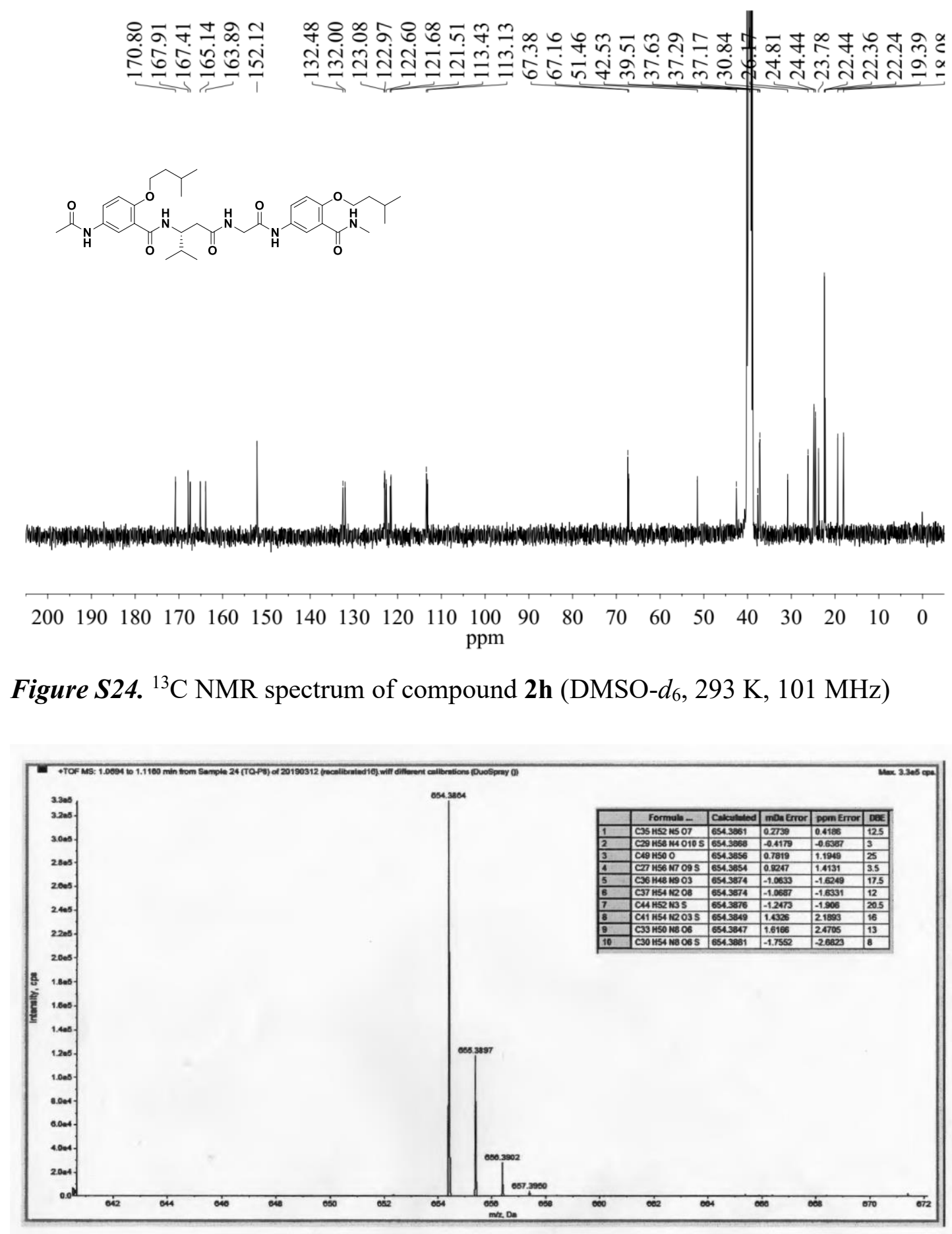

Figure S25. MS spectrum of compound $\mathbf{2 h}$ 

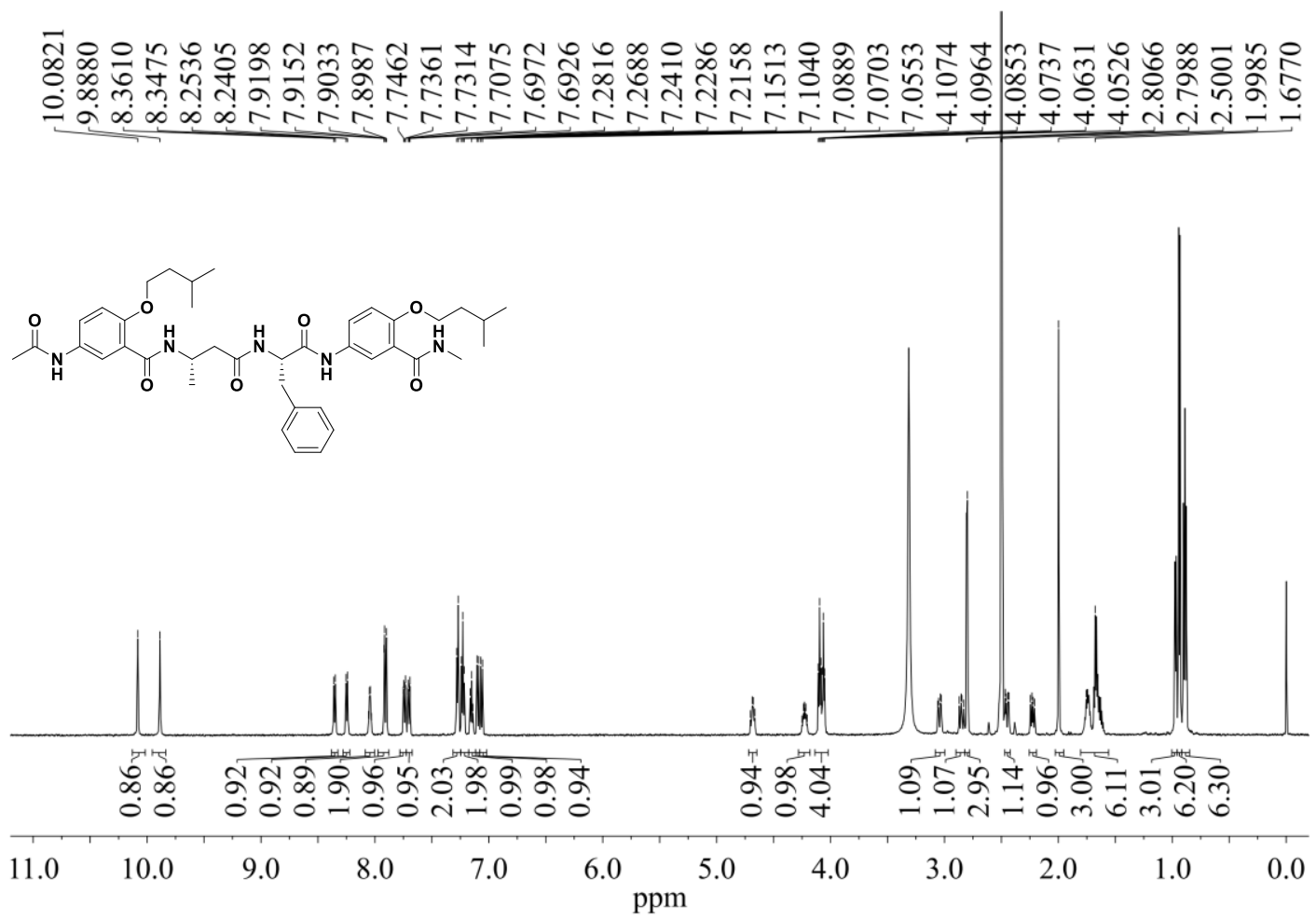

Figure S26. ${ }^{1} \mathrm{H}$ NMR spectrum of compound $2 \mathbf{i}\left(\mathrm{DMSO}-d_{6}, 298 \mathrm{~K}, 600 \mathrm{MHz}\right)$

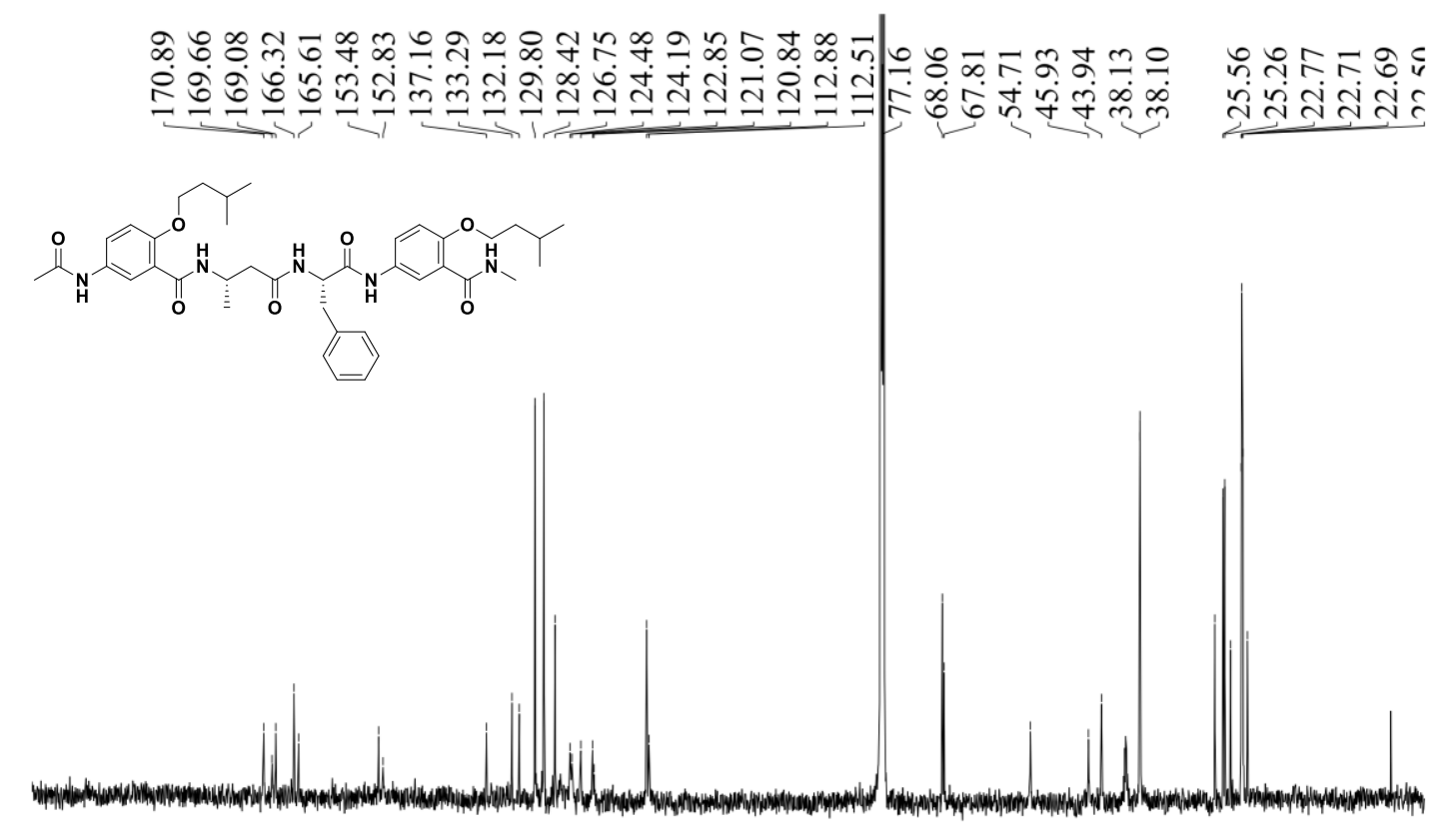

$\begin{array}{lllllllllllllllllllll}200 & 190 & 180 & 170 & 160 & 150 & 140 & 130 & 120 & 110 & \begin{array}{c}100 \\ \mathrm{ppm}\end{array} & 90 & 80 & 70 & 60 & 50 & 40 & 30 & 20 & 10 & 0\end{array}$

Figure S27. ${ }^{13} \mathrm{C}$ NMR spectrum of compound $2 \mathbf{i}\left(\mathrm{CDCl}_{3}, 299 \mathrm{~K}, 151 \mathrm{MHz}\right)$ 


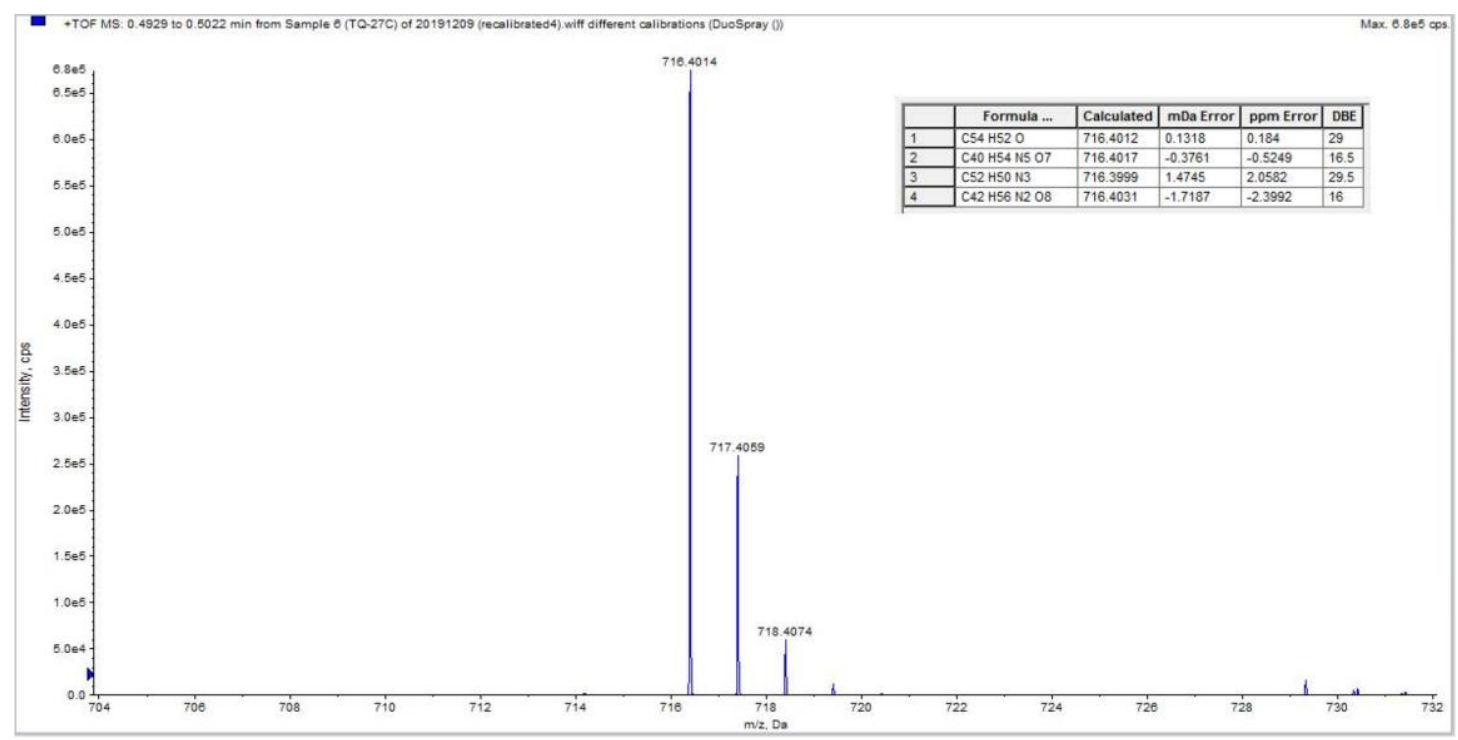

Figure $\mathbf{S 2 8 .}$ MS spectrum of compound $2 \mathbf{i}$
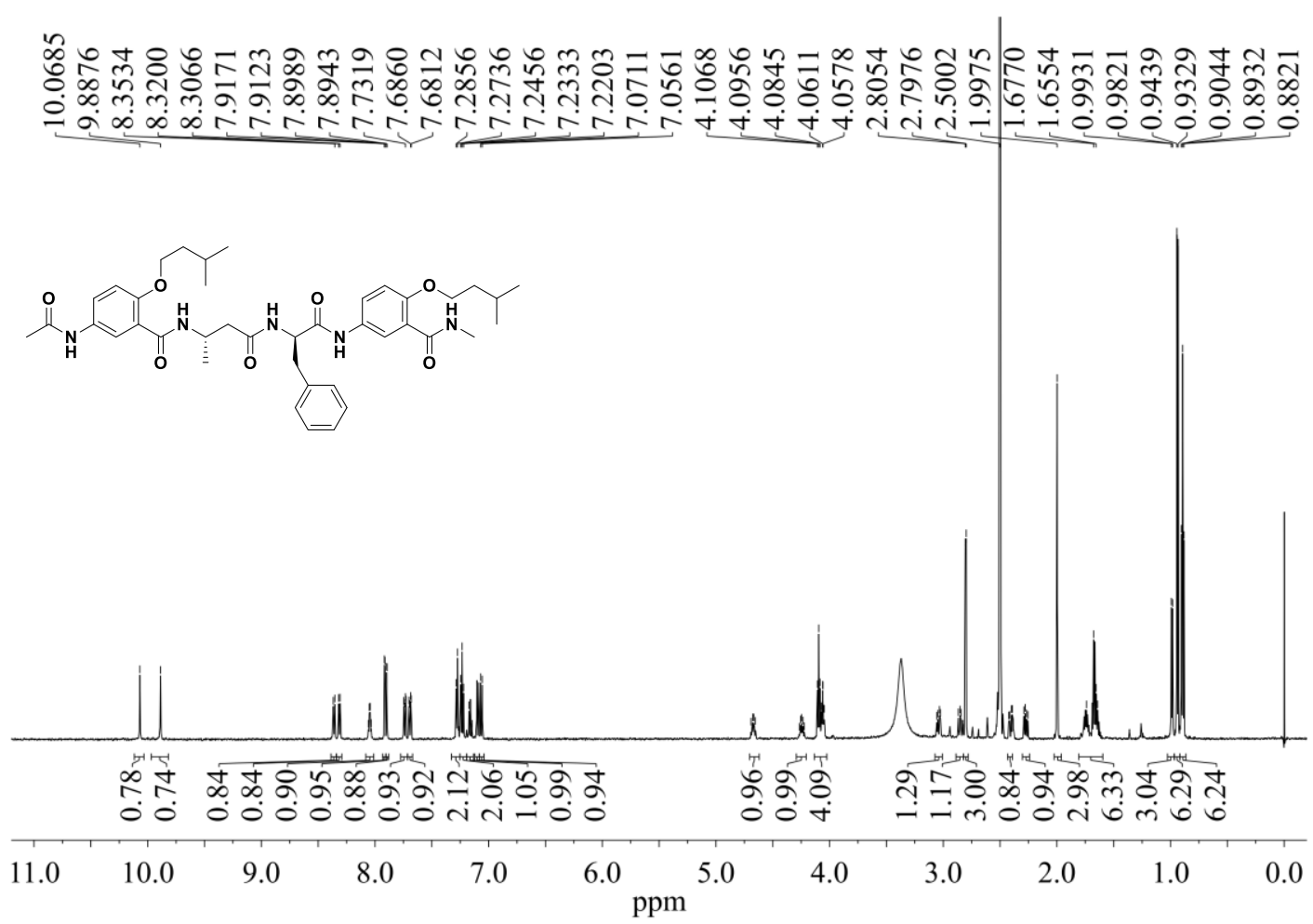

Figure S29. ${ }^{1} \mathrm{H}$ NMR spectrum of compound 2j (DMSO- $d_{6}, 297 \mathrm{~K}, 600 \mathrm{MHz}$ ) 


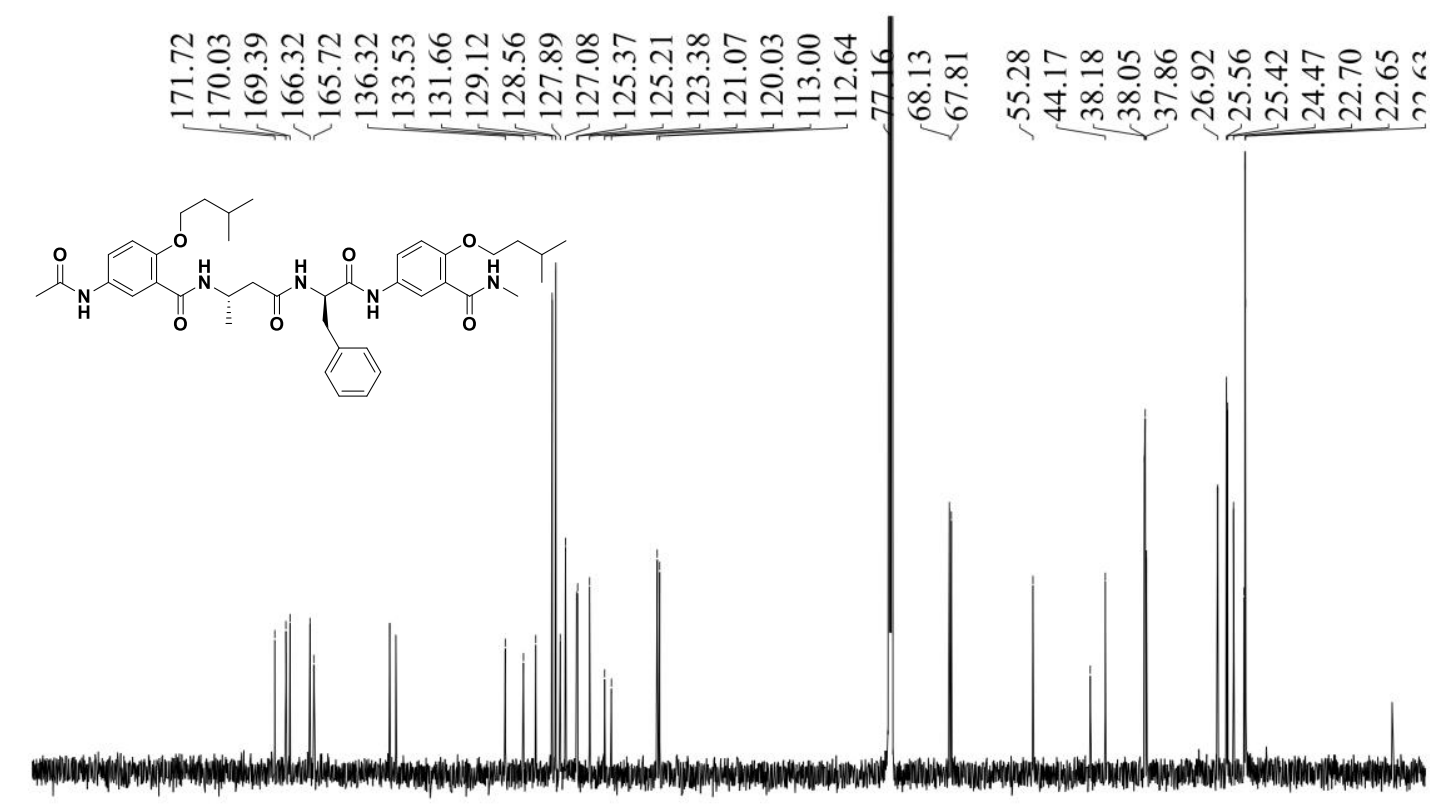

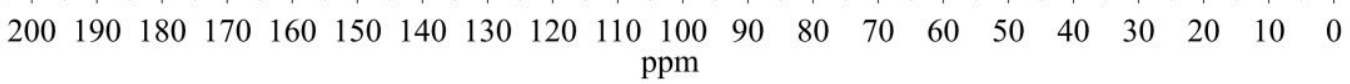

Figure S30. ${ }^{13} \mathrm{C}$ NMR spectrum of compound $2 \mathbf{j}\left(\mathrm{CDCl}_{3}, 297 \mathrm{~K}, 151 \mathrm{MHz}\right)$

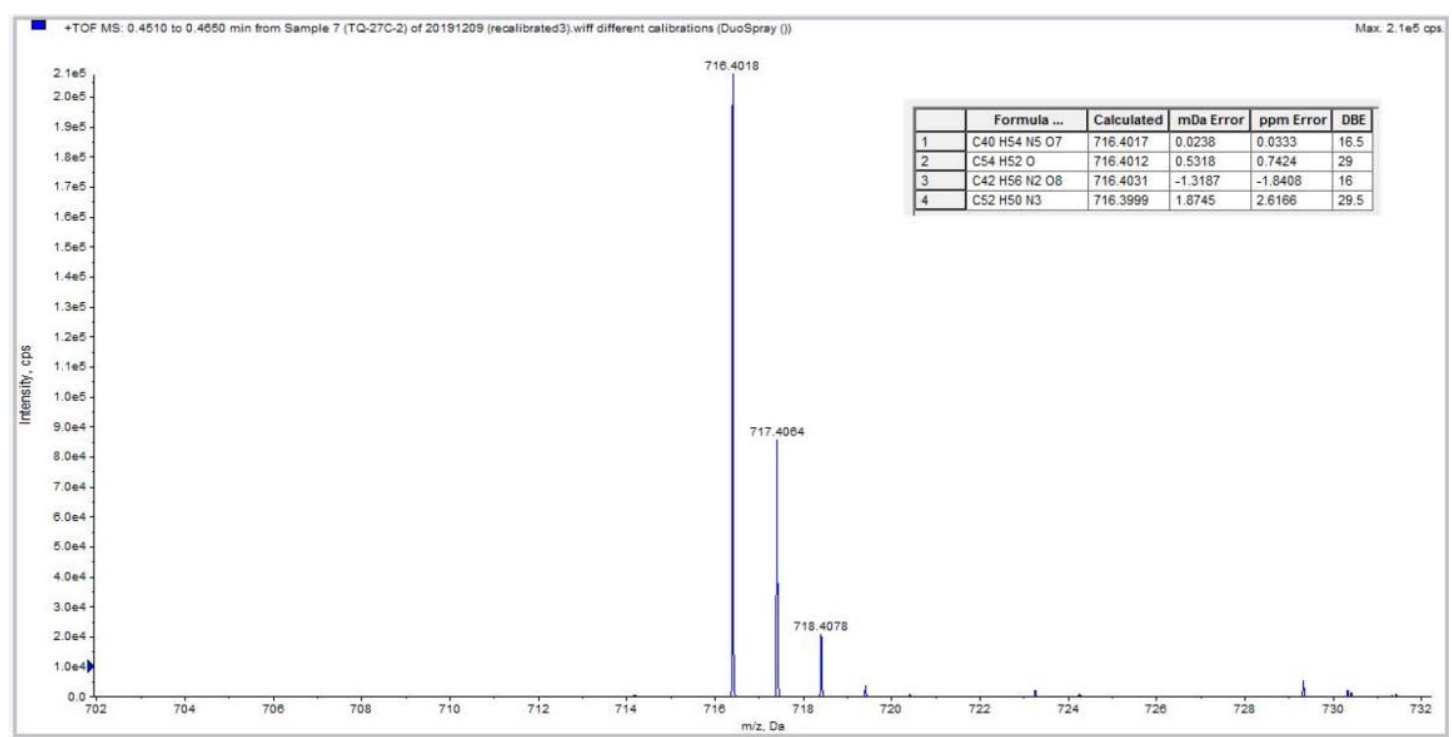

Figure S31. MS spectrum of compound $\mathbf{2 j}$ 

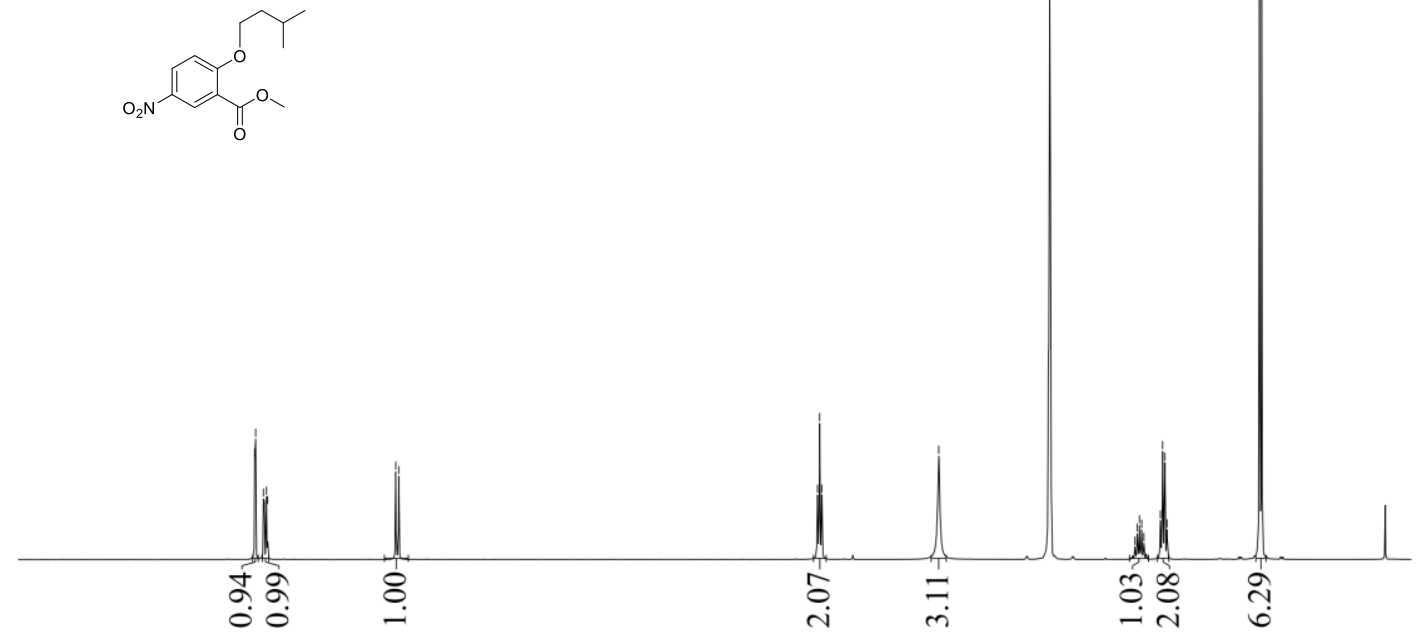

$\begin{array}{lllllllllllllllllllll}10.0 & 9.5 & 9.0 & 8.5 & 8.0 & 7.5 & 7.0 & 6.5 & 6.0 & 5.5 & \begin{array}{c}5.0 \\ \mathrm{ppm}\end{array} & 4.5 & 4.0 & 3.5 & 3.0 & 2.5 & 2.0 & 1.5 & 1.0 & 0.5 & 0.0\end{array}$

Figure S32. ${ }^{1} \mathrm{H}$ NMR spectra of compound 7 (DMSO-d $d_{6}, 296 \mathrm{~K}, 400 \mathrm{MHz}$ )

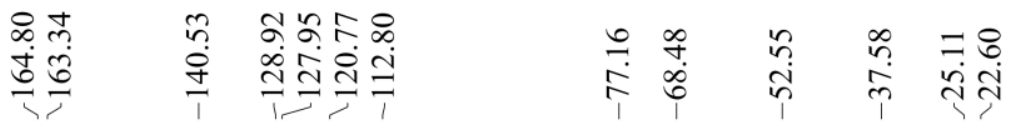

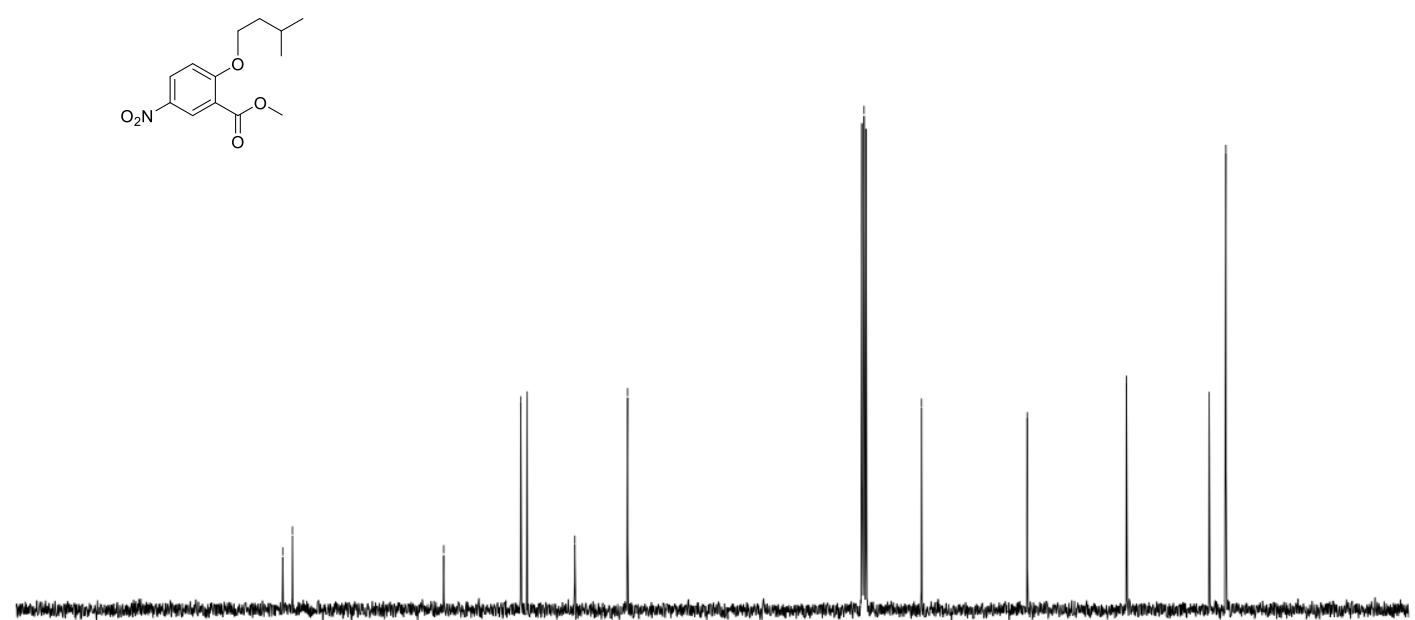

$\begin{array}{lllllllllllllllllllll}200 & 190 & 180 & 170 & 160 & 150 & 140 & 130 & 120 & 110 & 100 & 90 & 80 & 70 & 60 & 50 & 40 & 30 & 20 & 10 & 0\end{array}$

Figure S33. ${ }^{13} \mathrm{C}$ NMR spectra of compound $7\left(\mathrm{CDCl}_{3}, 298 \mathrm{~K}, 101 \mathrm{MHz}\right)$ 

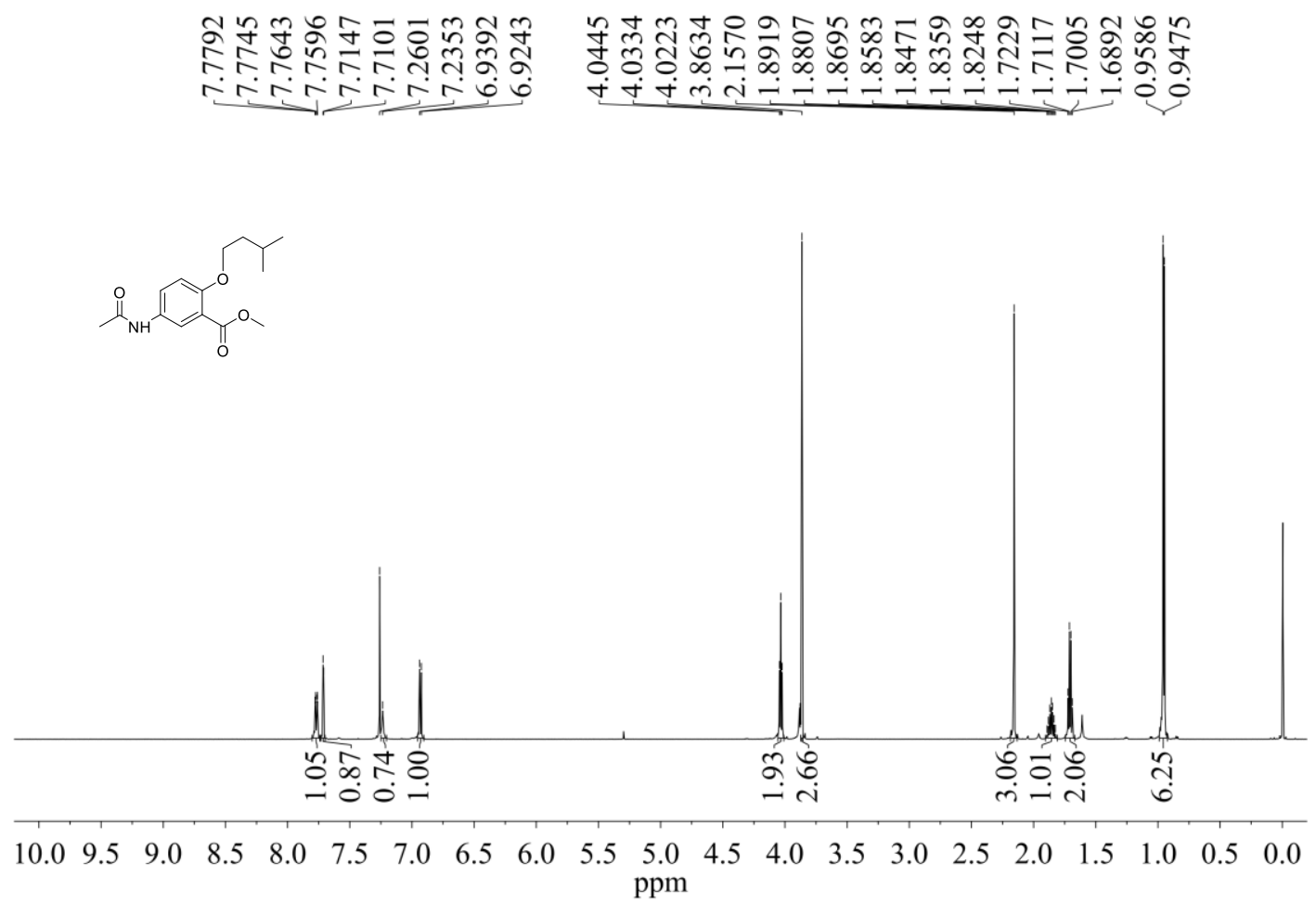

Figure S34. ${ }^{1} \mathrm{H}$ NMR spectra of compound $9\left(\mathrm{CDCl}_{3}, 293 \mathrm{~K}, 600 \mathrm{MHz}\right)$

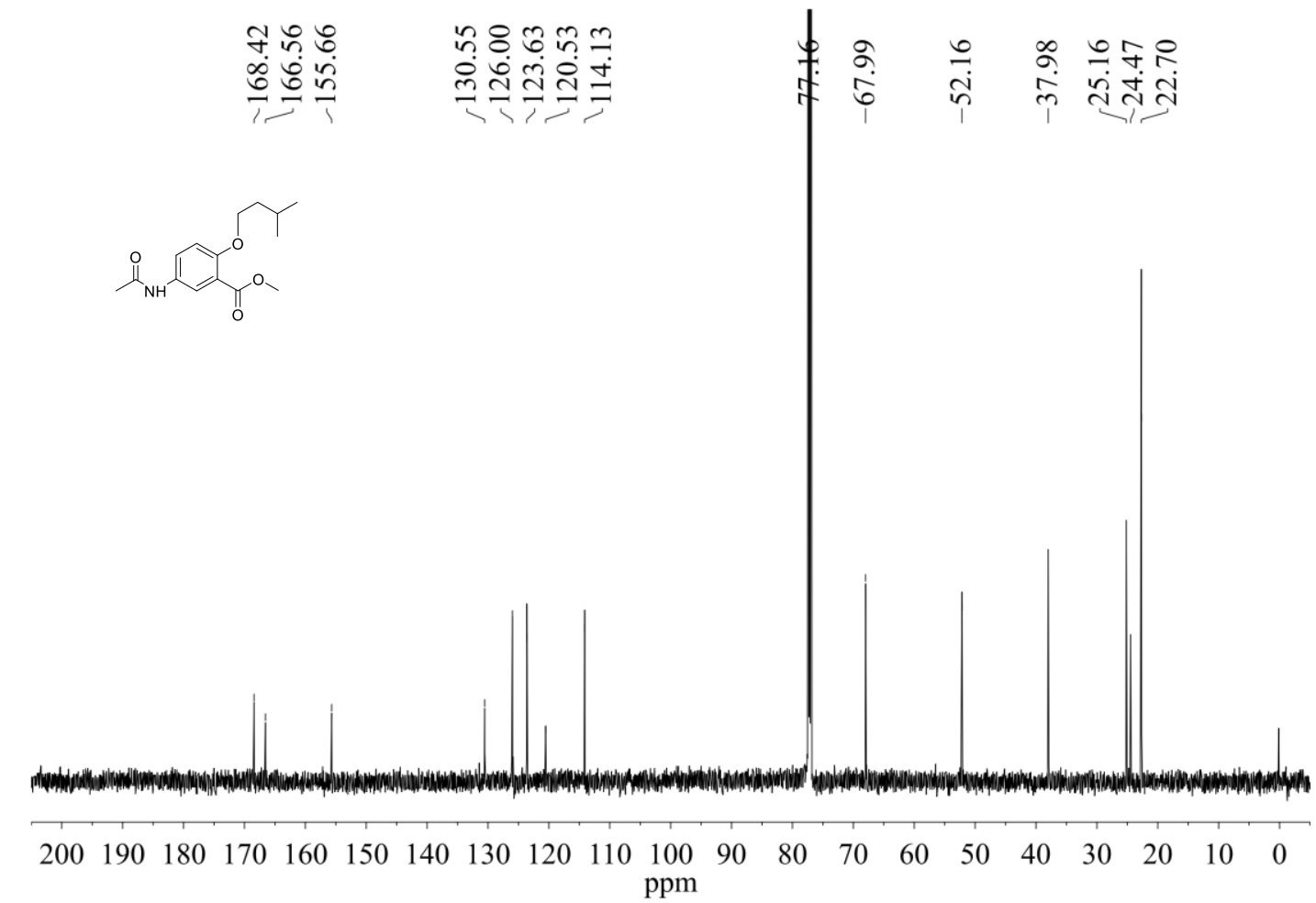

Figure S35. ${ }^{13} \mathrm{C}$ NMR spectra of compound $9\left(\mathrm{CDCl}_{3}, 296 \mathrm{~K}, 151 \mathrm{MHz}\right)$ 

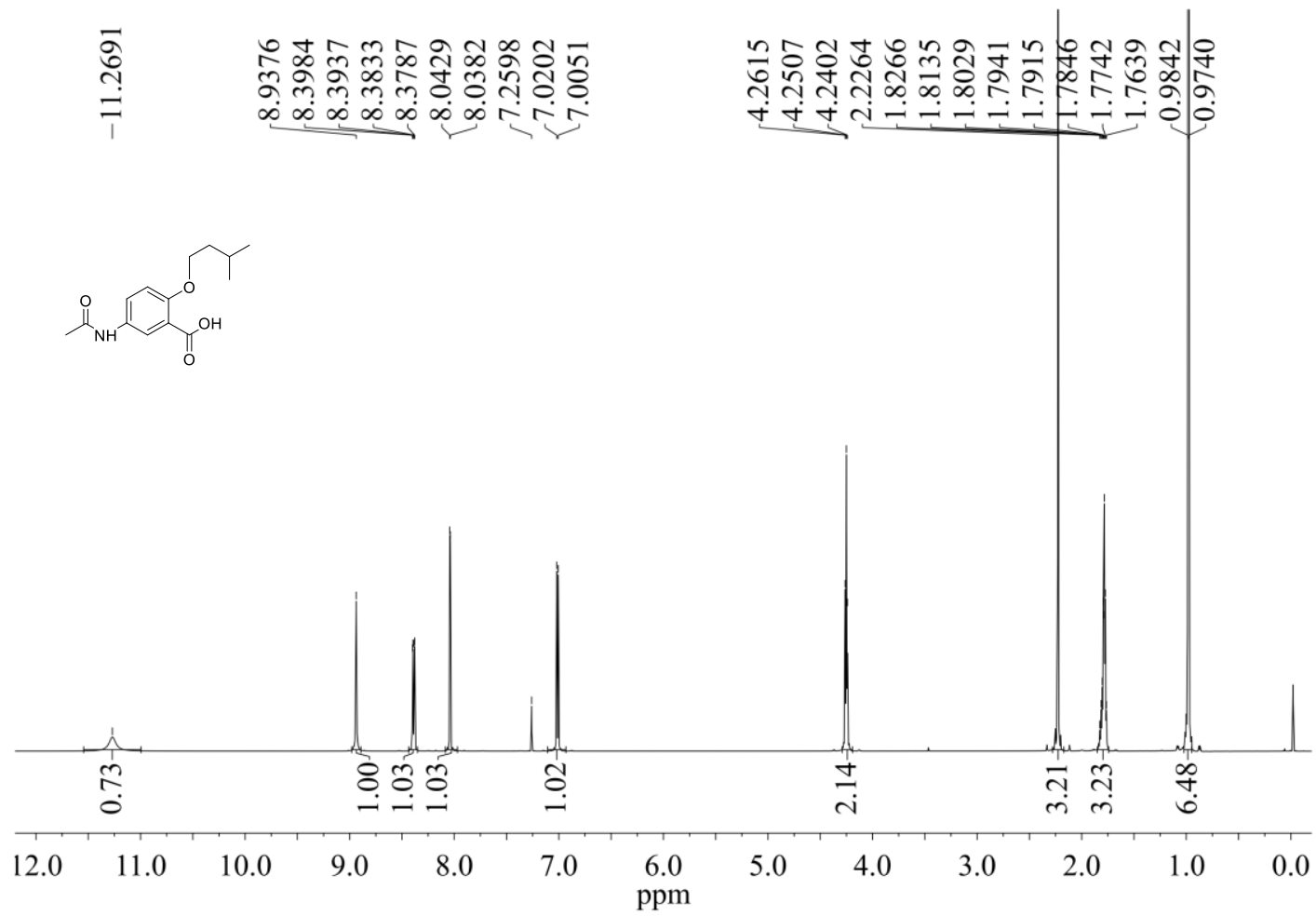

Figure S36. ${ }^{1} \mathrm{H}$ NMR spectra of compound $10\left(\mathrm{CDCl}_{3}, 293 \mathrm{~K}, 600 \mathrm{MHz}\right)$
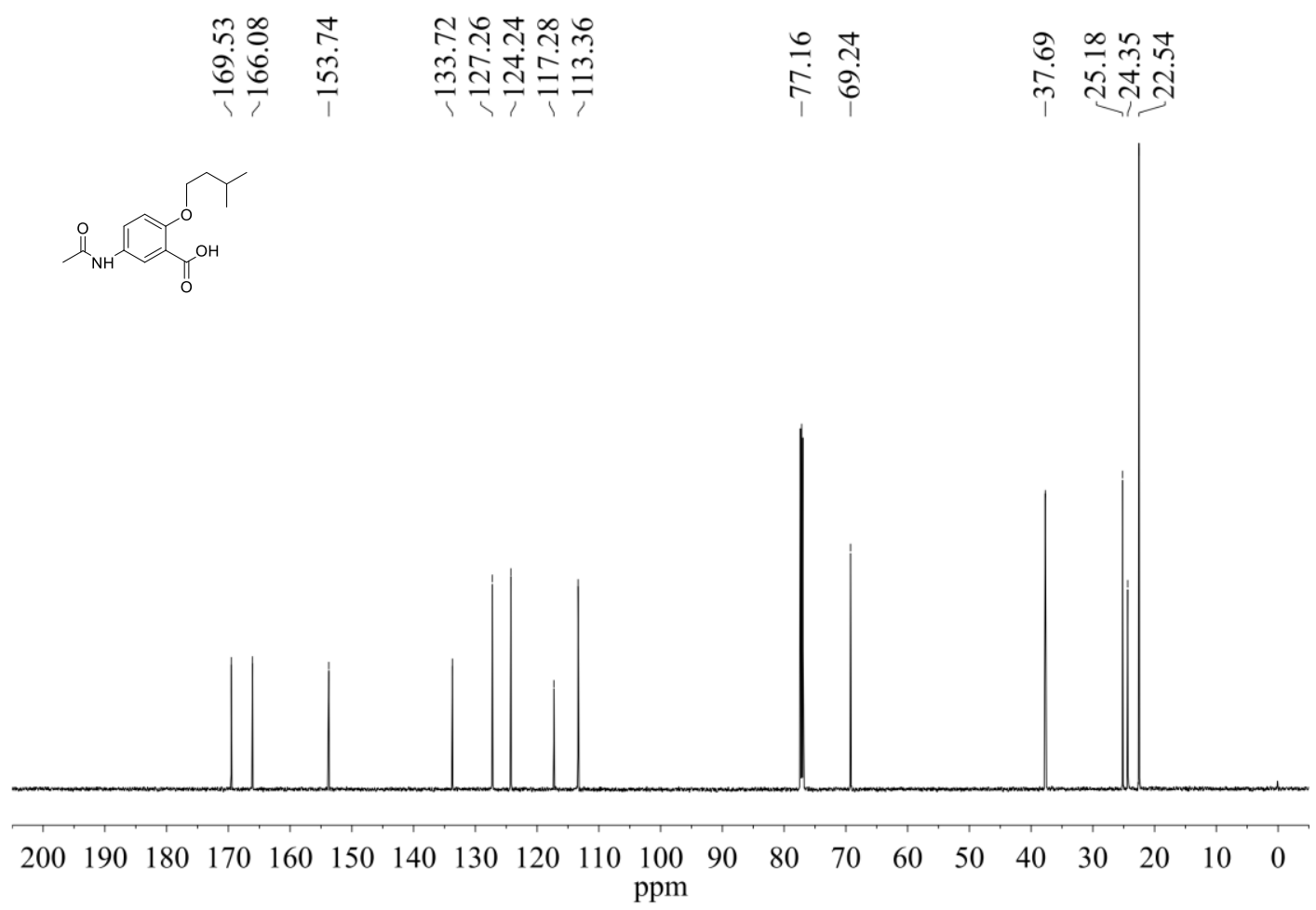

Figure S37. ${ }^{13} \mathrm{C}$ NMR spectra of compound $10\left(\mathrm{CDCl}_{3}, 295 \mathrm{~K}, 151 \mathrm{MHz}\right)$ 


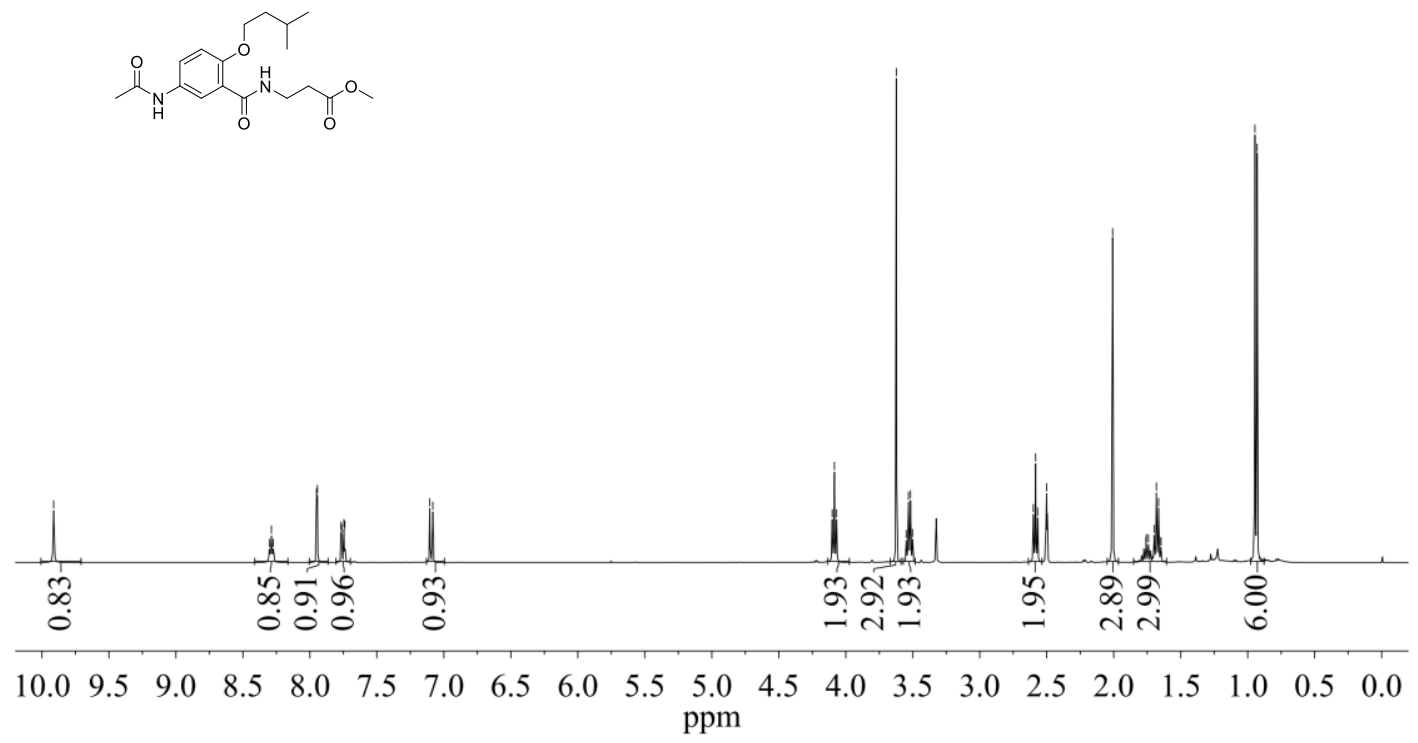

Figure S38. ${ }^{1} \mathrm{H}$ NMR spectra of compound 11 (DMSO-d $\left.6,298 \mathrm{~K}, 400 \mathrm{MHz}\right)$
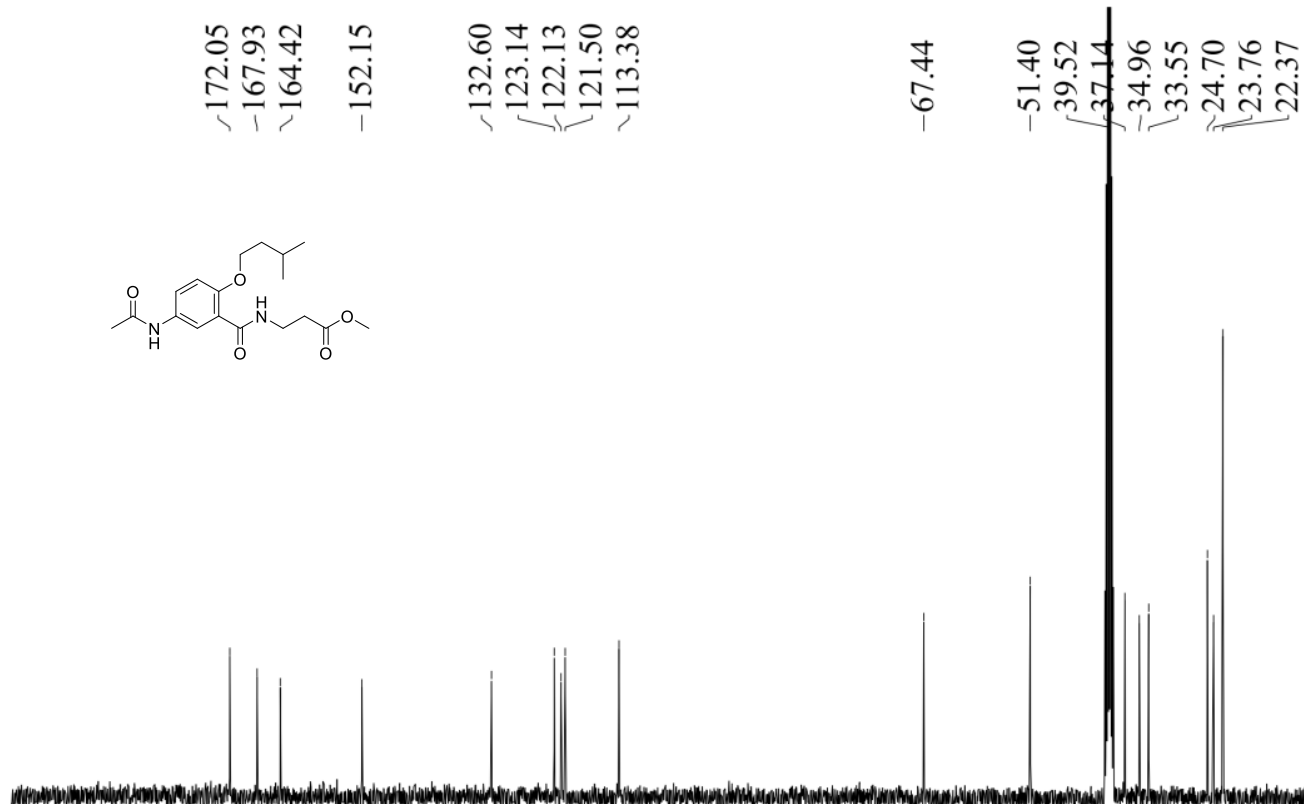

$\begin{array}{lllllllllllllllllllll}200 & 190 & 180 & 170 & 160 & 150 & 140 & 130 & 120 & 110 & \begin{array}{c}100 \\ \mathrm{ppm}\end{array} & 90 & 80 & 70 & 60 & 50 & 40 & 30 & 20 & 10 & 0\end{array}$

Figure S39. ${ }^{13} \mathrm{C}$ NMR spectra of compound 11 (DMSO- $\left.d_{6}, 298 \mathrm{~K}, 151 \mathrm{MHz}\right)$ 


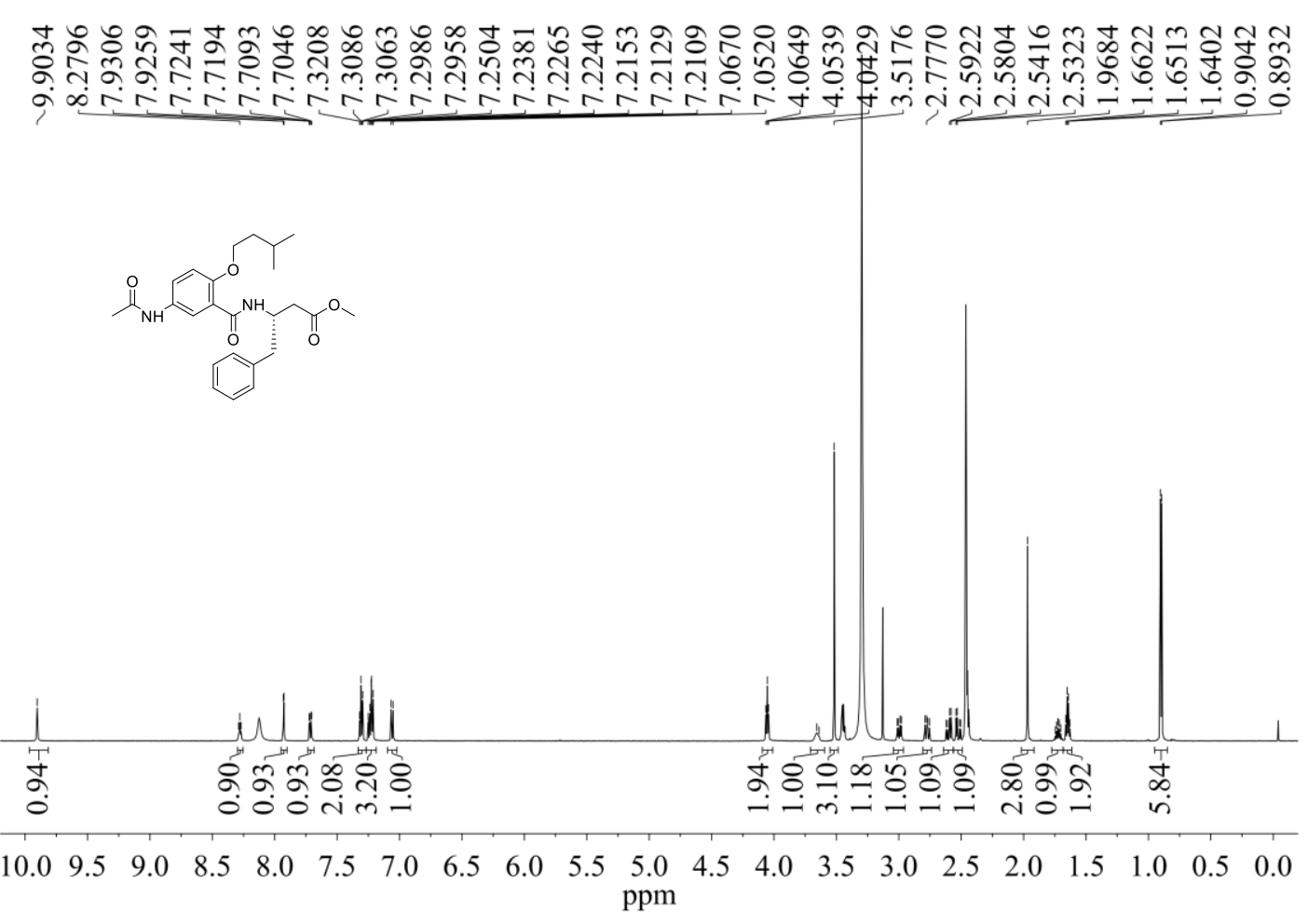

Figure S40. ${ }^{1} \mathrm{H}$ NMR spectra of compound 12 (DMSO-d $d_{6}, 298 \mathrm{~K}, 600 \mathrm{MHz}$ )

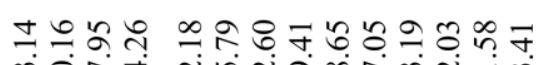

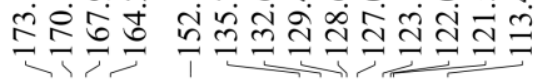

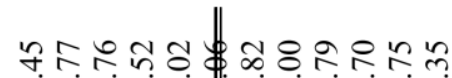

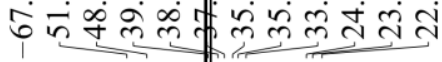
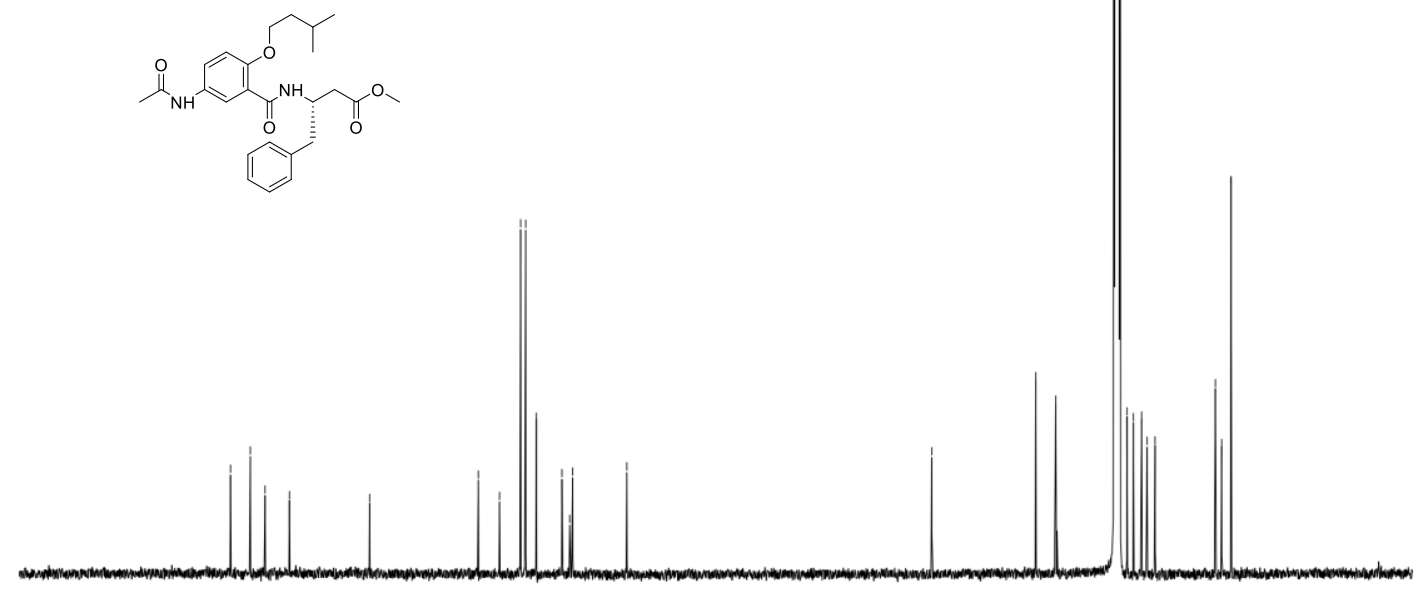

$\begin{array}{lllllllllllllllllllll}200 & 190 & 180 & 170 & 160 & 150 & 140 & 130 & 120 & 110 & 100 & 90 & 80 & 70 & 60 & 50 & 40 & 30 & 20 & 10 & 0\end{array}$

Figure S41. ${ }^{13} \mathrm{C}$ NMR spectra of compound 12 (DMSO- $\left.d_{6}, 300 \mathrm{~K}, 151 \mathrm{MHz}\right)$ 


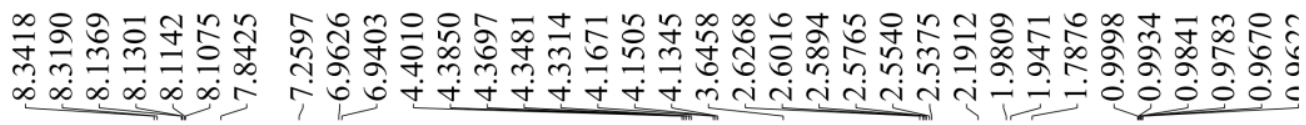

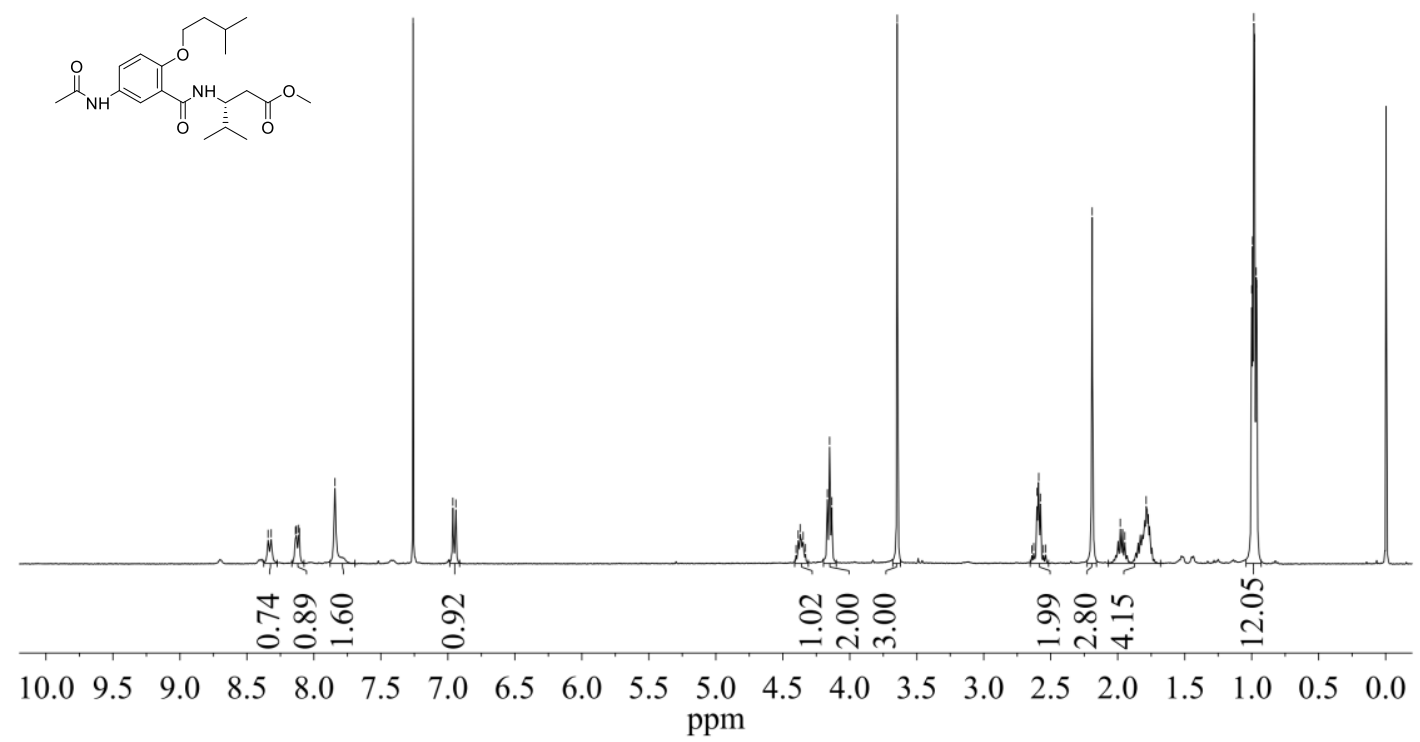

Figure S42. ${ }^{1} \mathrm{H}$ NMR spectra of compound $13\left(\mathrm{CDCl}_{3}, 296 \mathrm{~K}, 400 \mathrm{MHz}\right)$
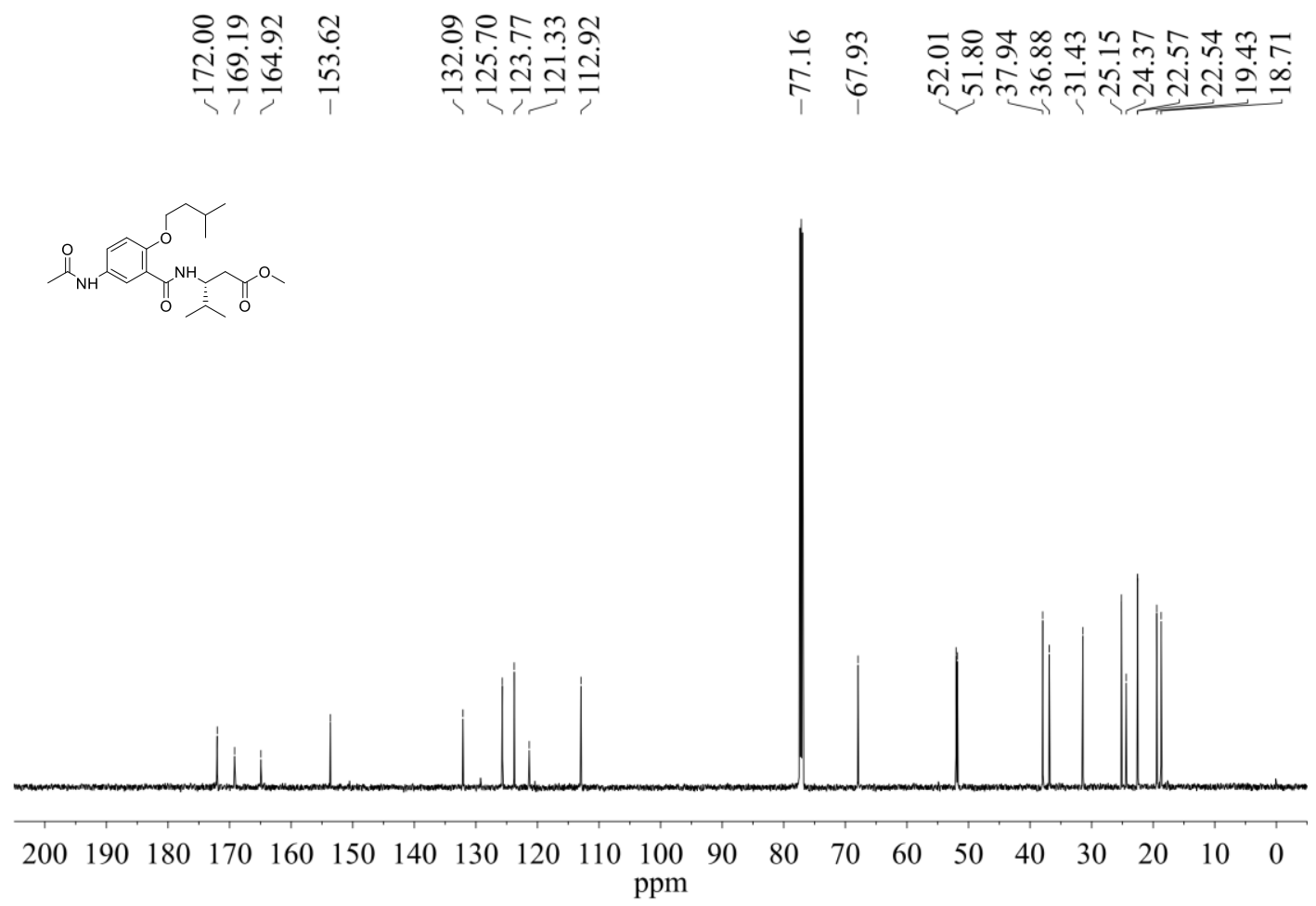

Figure S43. ${ }^{13} \mathrm{C}$ NMR spectra of compound $13\left(\mathrm{CDCl}_{3}, 296 \mathrm{~K}, 151 \mathrm{MHz}\right)$ 


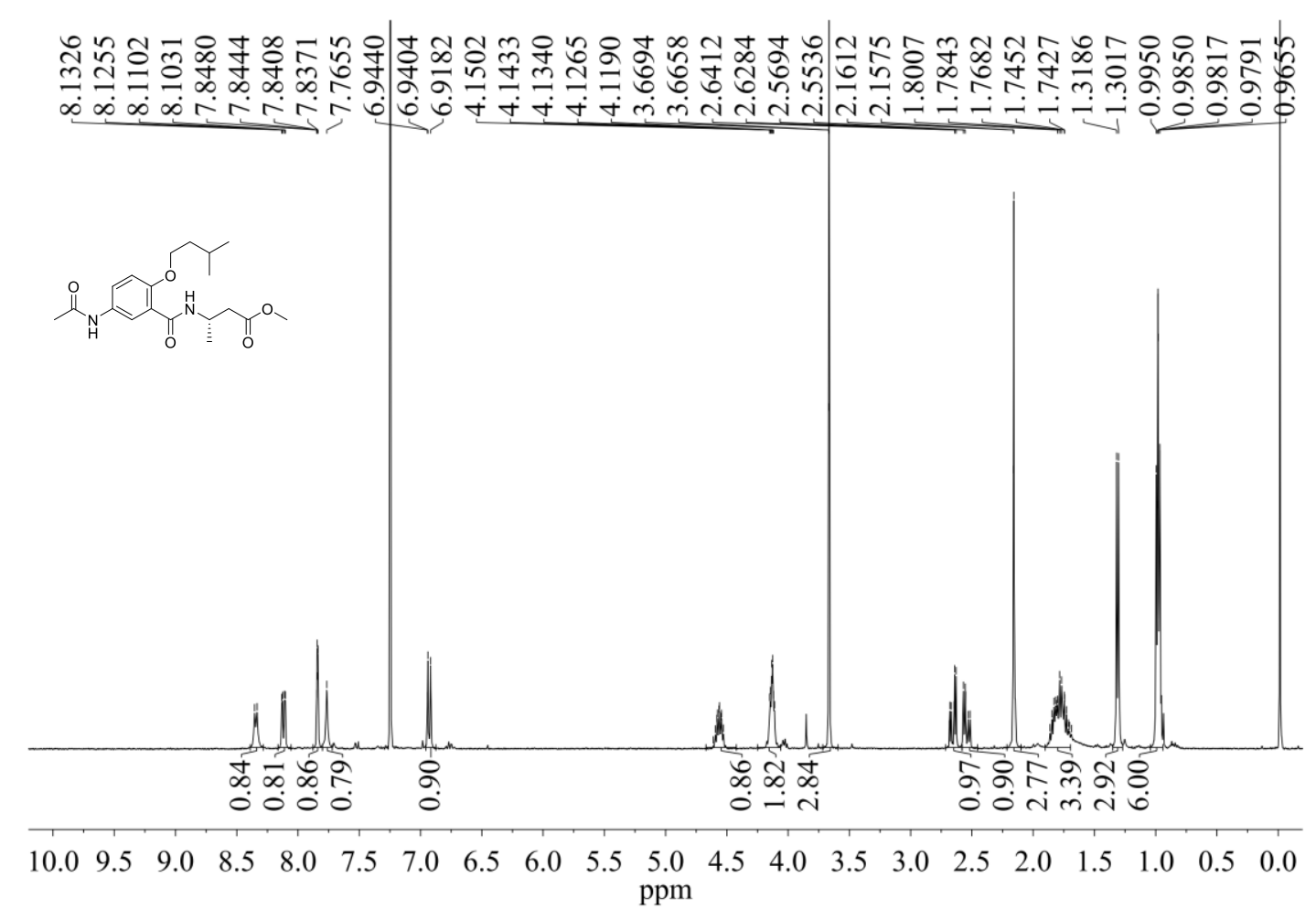

Figure S44. ${ }^{1} \mathrm{H}$ NMR spectra of compound $14\left(\mathrm{CDCl}_{3}, 298 \mathrm{~K}, 400 \mathrm{MHz}\right)$
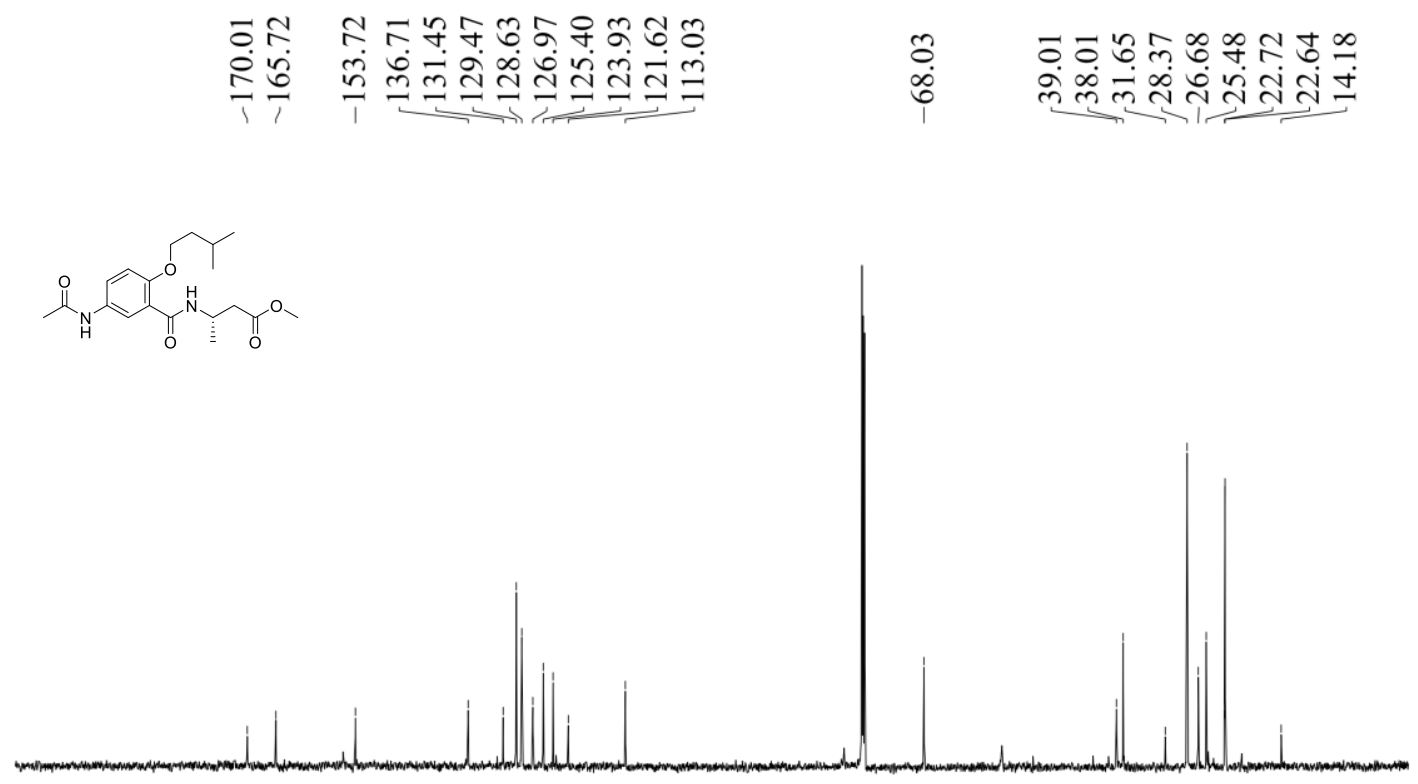

$\begin{array}{llllllllllllllllllllllll}200 & 190 & 180 & 170 & 160 & 150 & 140 & 130 & 120 & 110 & 100 & 90 & 80 & 70 & 60 & 50 & 40 & 30 & 20 & 10 & 0\end{array}$

Figure S45. ${ }^{13} \mathrm{C}$ NMR spectra of compound $14\left(\mathrm{CDCl}_{3}, 299 \mathrm{~K}, 151 \mathrm{MHz}\right)$ 

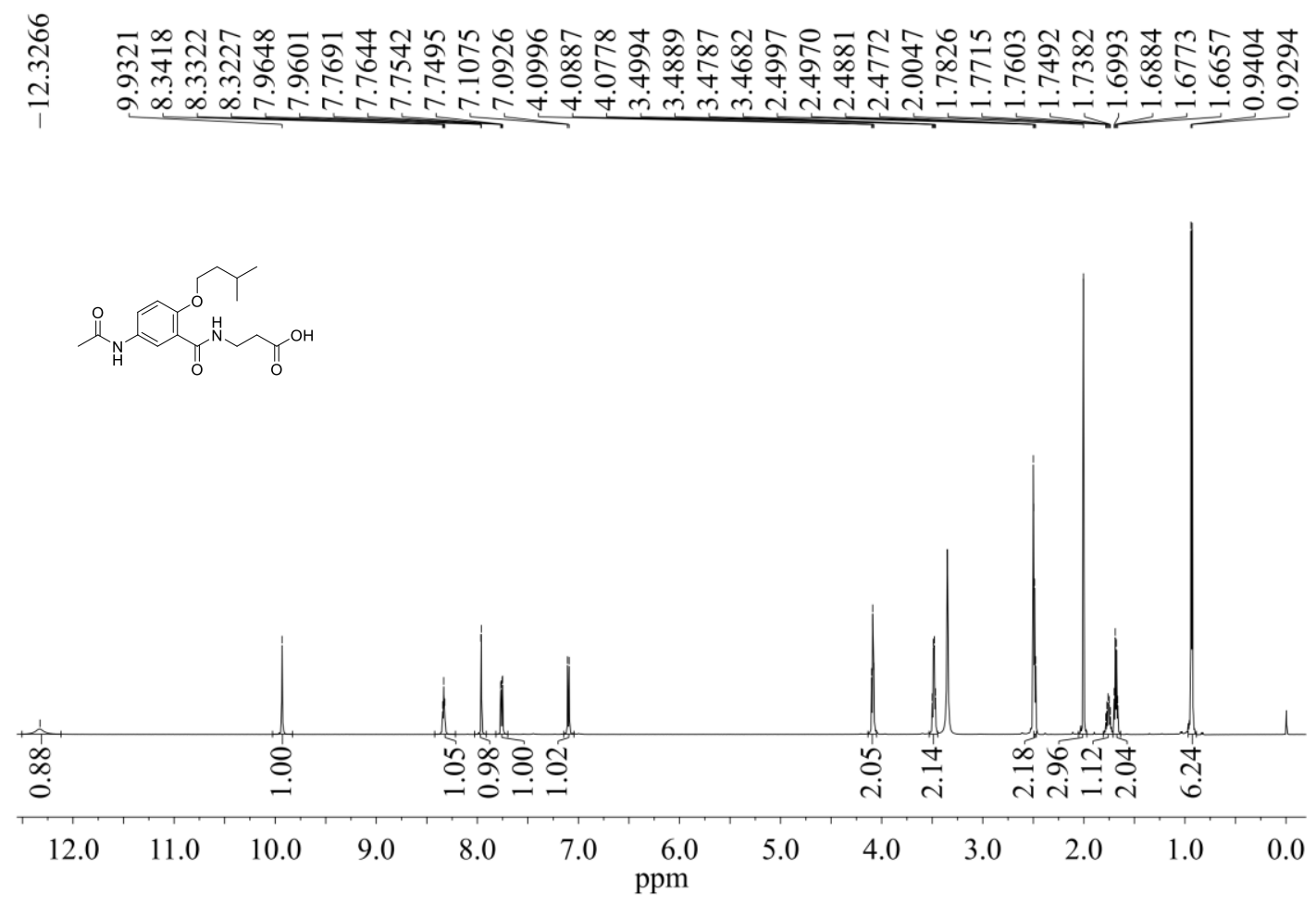

Figure S46. ${ }^{1} \mathrm{H}$ NMR spectra of compound 15 (DMSO-d $d_{6}, 293 \mathrm{~K}, 600 \mathrm{MHz}$ )
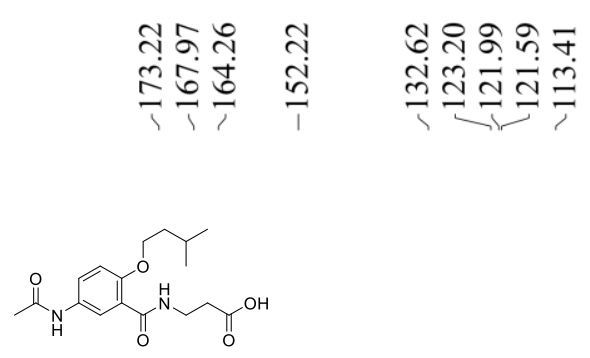

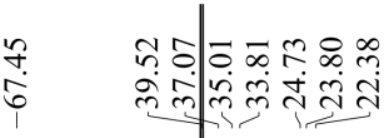

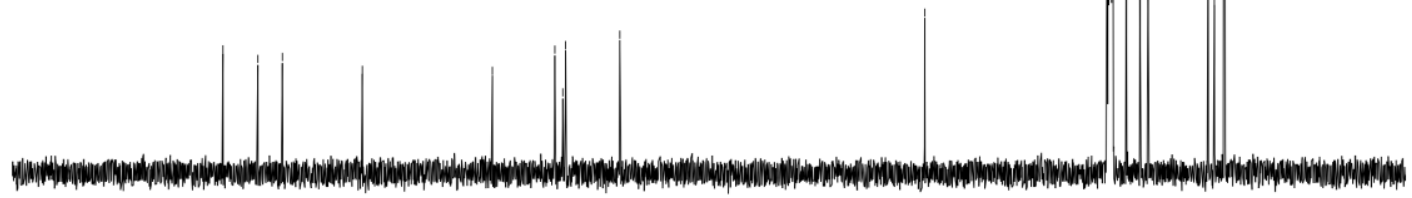

$\begin{array}{lllllllllllllllllllll}200 & 190 & 180 & 170 & 160 & 150 & 140 & 130 & 120 & 110 & 100 & 90 & 80 & 70 & 60 & 50 & 40 & 30 & 20 & 10 & 0\end{array}$

Figure S47. ${ }^{13} \mathrm{C}$ NMR spectra of compound 15 (DMSO- $d_{6}, 295 \mathrm{~K}, 151 \mathrm{MHz}$ ) 


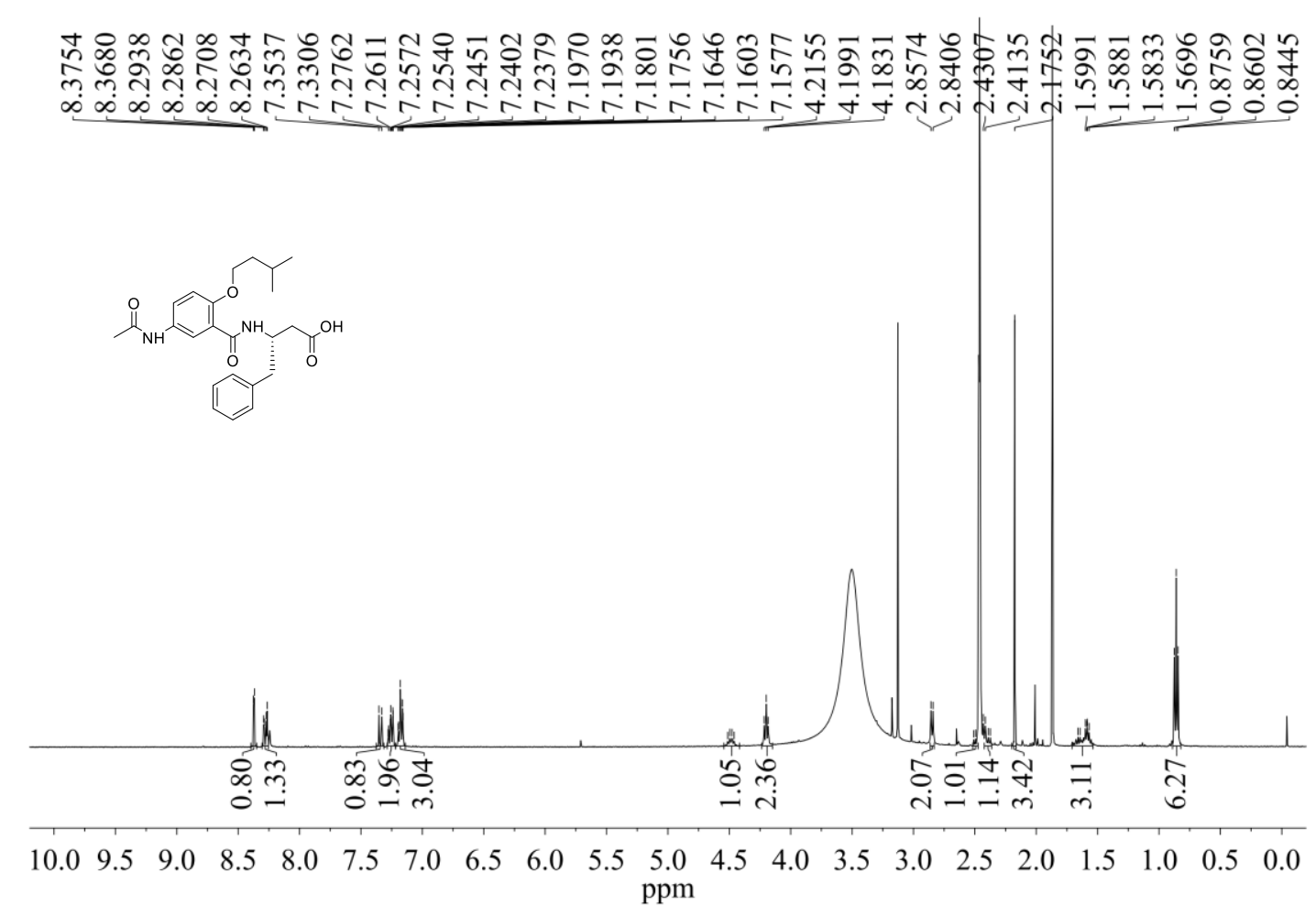

Figure S48. ${ }^{1} \mathrm{H}$ NMR spectra of compound 16 (DMSO-d $d_{6}, 298 \mathrm{~K}, 400 \mathrm{MHz}$ )
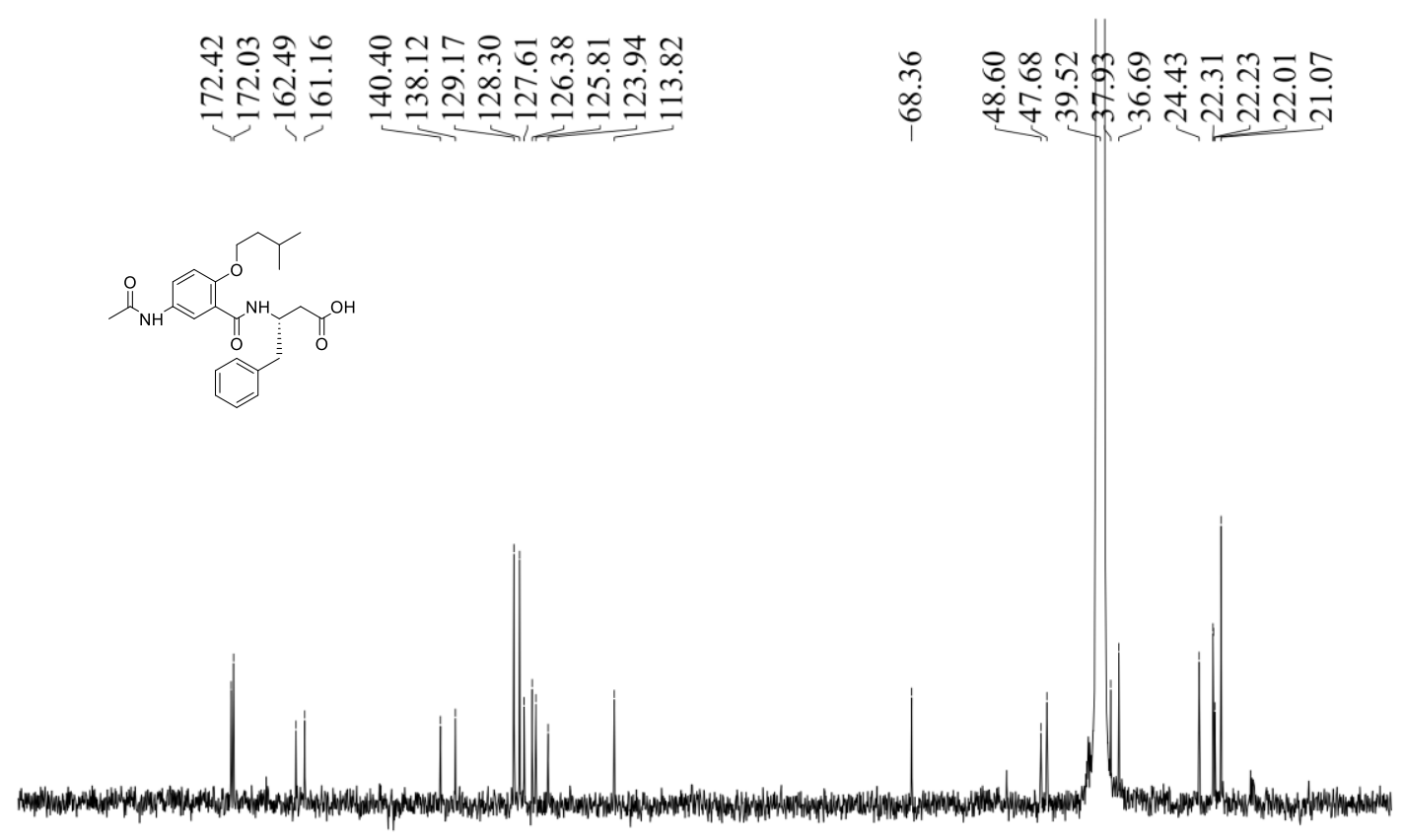

$\begin{array}{lllllllllllllllllllllll}200 & 190 & 180 & 170 & 160 & 150 & 140 & 130 & 120 & 110 & 100 & 90 & 80 & 70 & 60 & 50 & 40 & 30 & 20 & 10 & 0 \\ \mathrm{ppm} & & & & & & & \end{array}$

Figure S49. ${ }^{13} \mathrm{C}$ NMR spectra of compound 16 (DMSO- $\left.d_{6}, 298 \mathrm{~K}, 151 \mathrm{MHz}\right)$ 


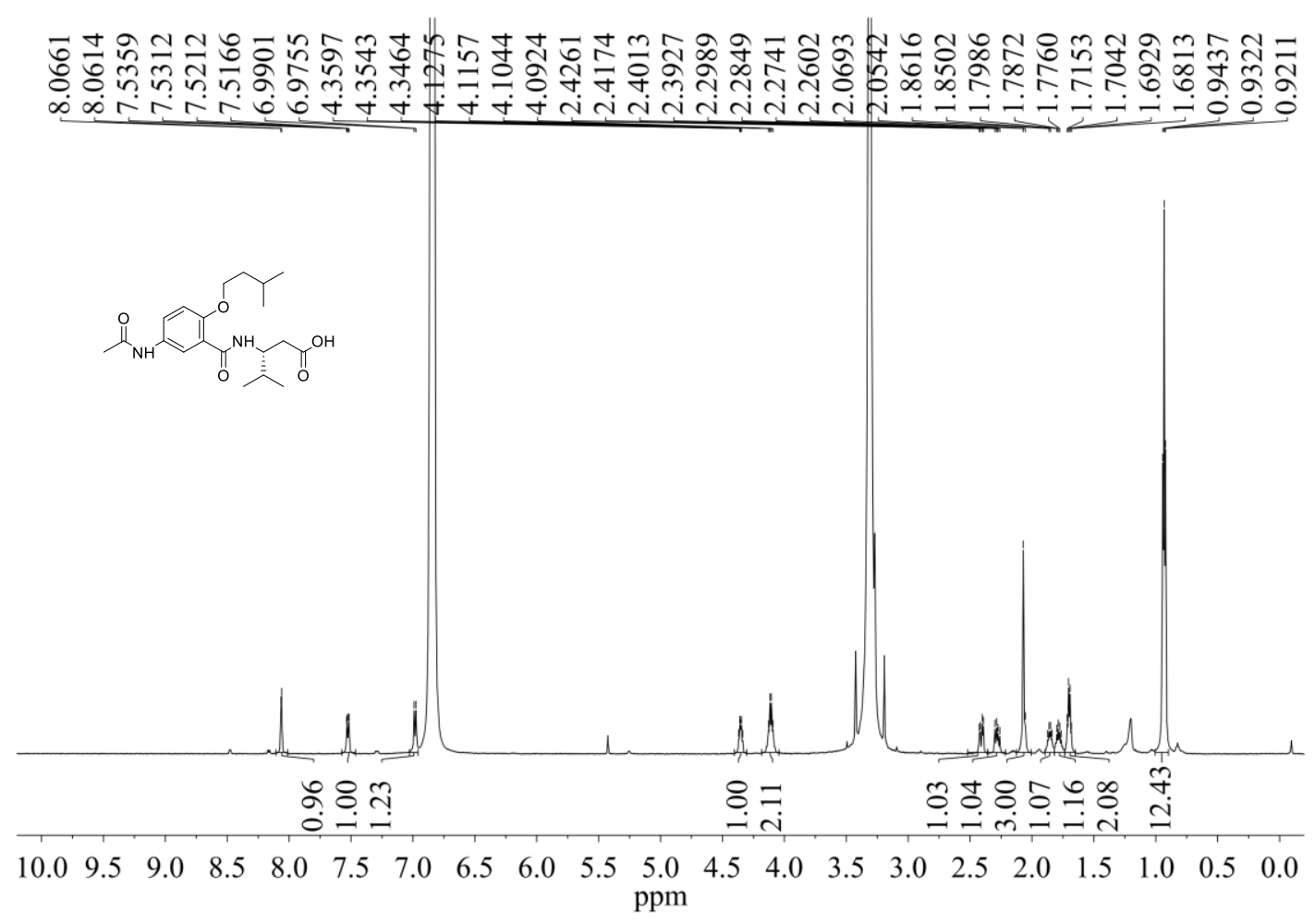

Figure S50. ${ }^{1} \mathrm{H}$ NMR spectra of compound 17 (Methol-d4, $303 \mathrm{~K}, 600 \mathrm{MHz}$ )

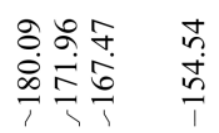

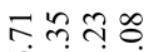
ปิบป

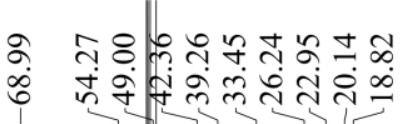
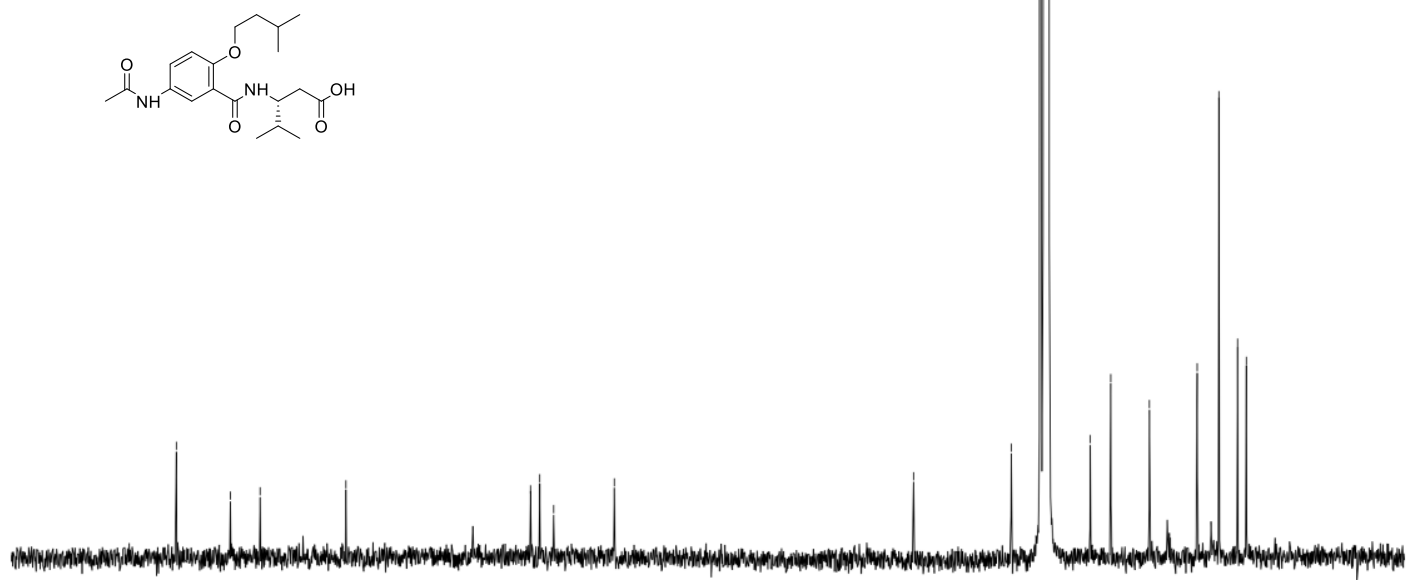

$\begin{array}{lllllllllllllllllllll}200 & 190 & 180 & 170 & 160 & 150 & 140 & 130 & 120 & 110 & 100 & 90 & 80 & 70 & 60 & 50 & 40 & 30 & 20 & 10 & 0\end{array}$

Figure S51. ${ }^{13} \mathrm{C}$ NMR spectra of compound 17 (Methol- $d 4,303 \mathrm{~K}, 151 \mathrm{MHz}$ ) 

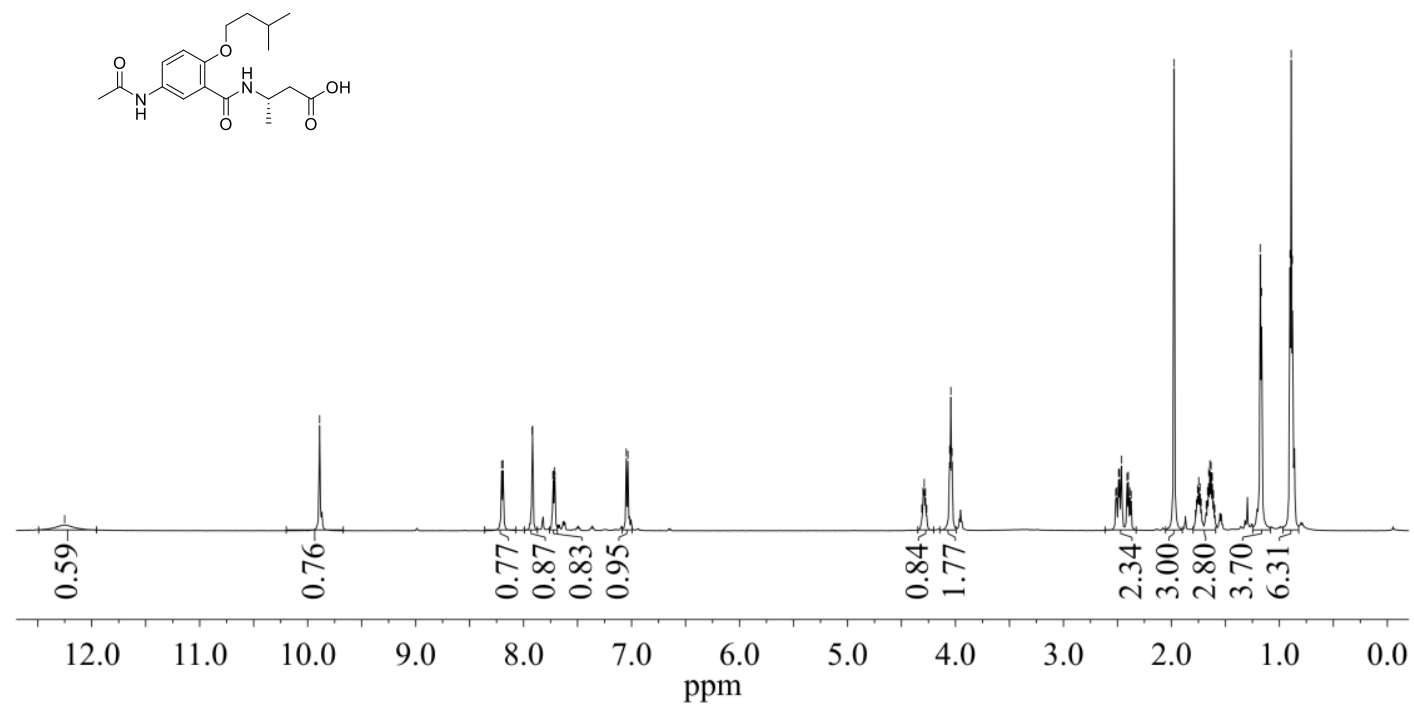

Figure S52. ${ }^{1} \mathrm{H}$ NMR spectra of compound 18 (DMSO- $\left.d_{6}, 298 \mathrm{~K}, 600 \mathrm{MHz}\right)$

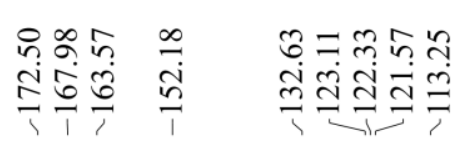
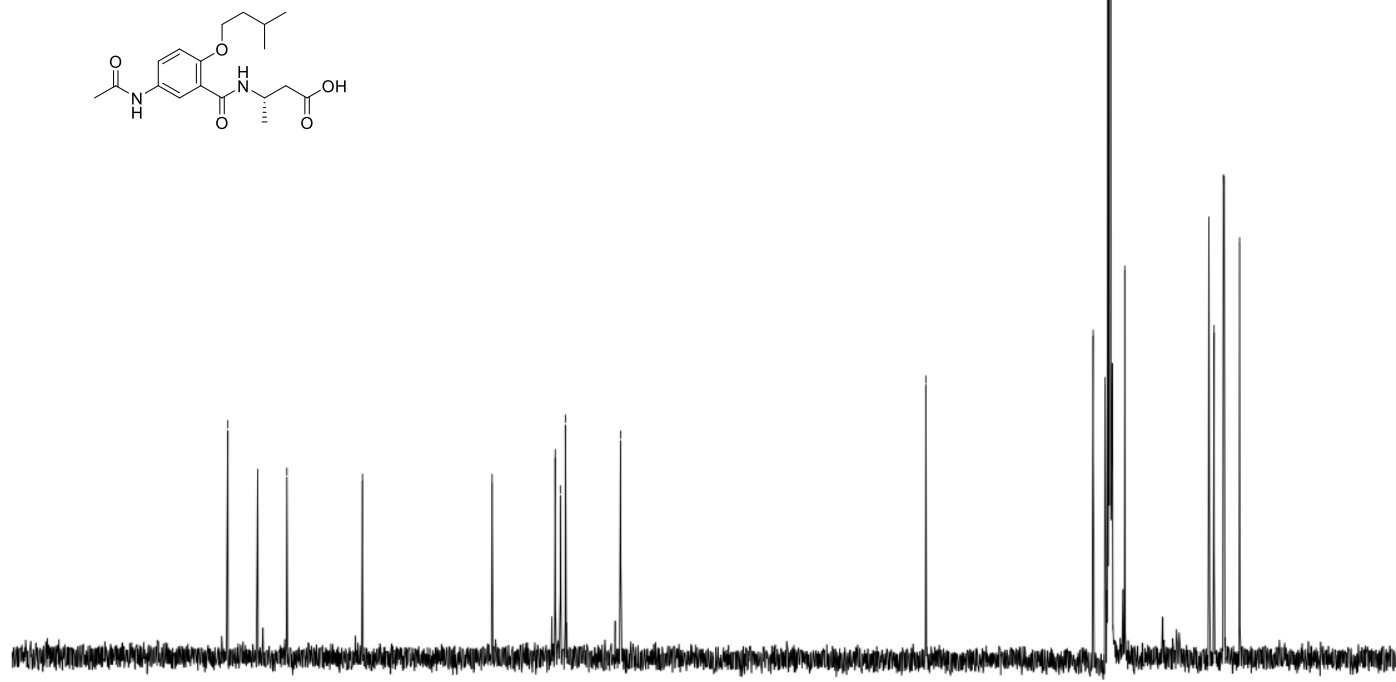

$\begin{array}{lllllllllllllllllllll}200 & 190 & 180 & 170 & 160 & 150 & 140 & 130 & 120 & 110 & 100 & 90 & 80 & 70 & 60 & 50 & 40 & 30 & 20 & 10 & 0 \\ \mathrm{ppm} & \end{array}$

Figure S53. ${ }^{13} \mathrm{C}$ NMR spectra of compound 18 (DMSO- $d_{6}, 299 \mathrm{~K}, 151 \mathrm{MHz}$ ) 

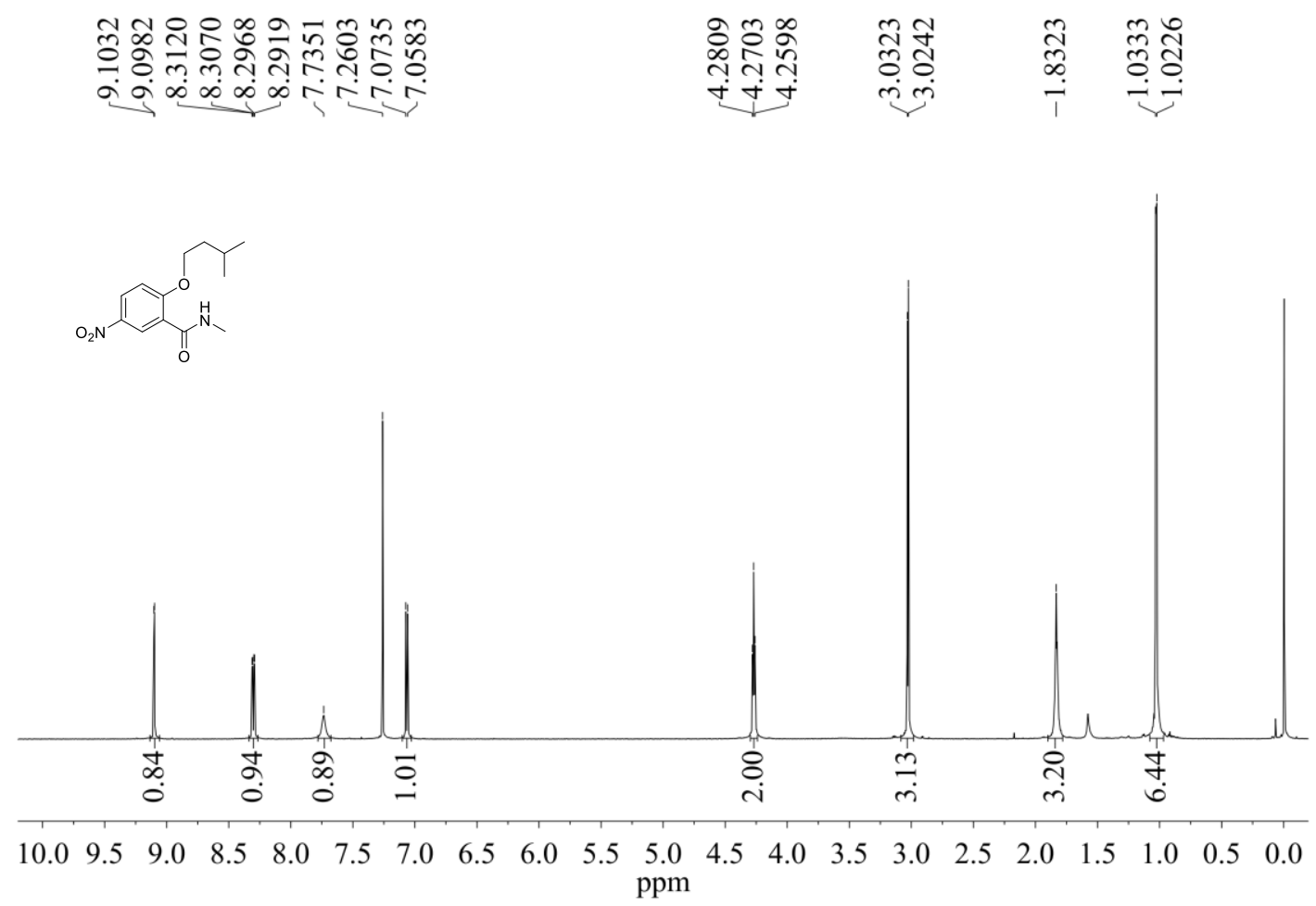

Figure S54. ${ }^{1} \mathrm{H}$ NMR spectra of compound $20\left(\mathrm{CDCl}_{3}, 293 \mathrm{~K}, 600 \mathrm{MHz}\right)$

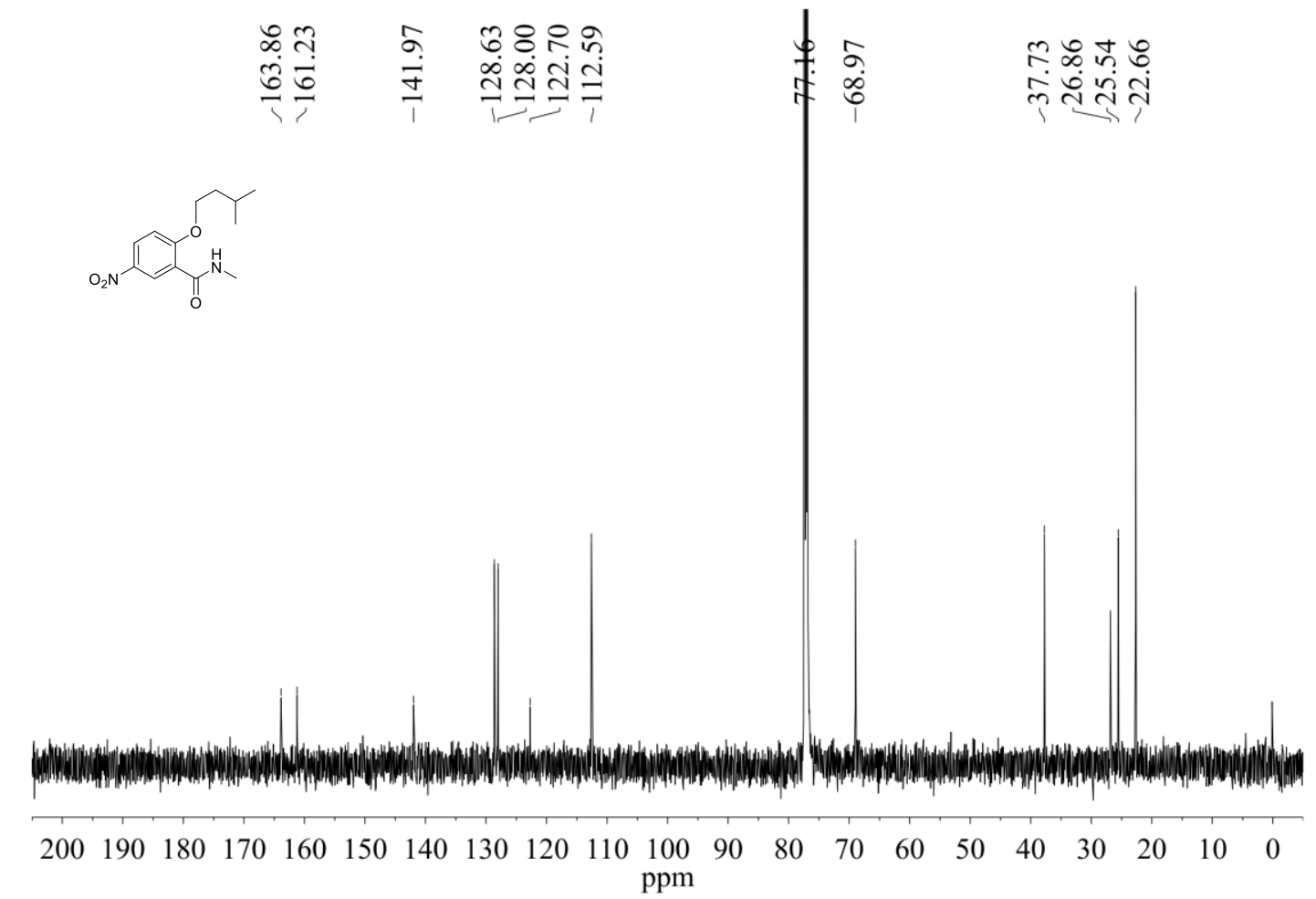

Figure S55. ${ }^{13} \mathrm{C}$ NMR spectra of compound $20\left(\mathrm{CDCl}_{3}, 295 \mathrm{~K}, 151 \mathrm{MHz}\right)$ 

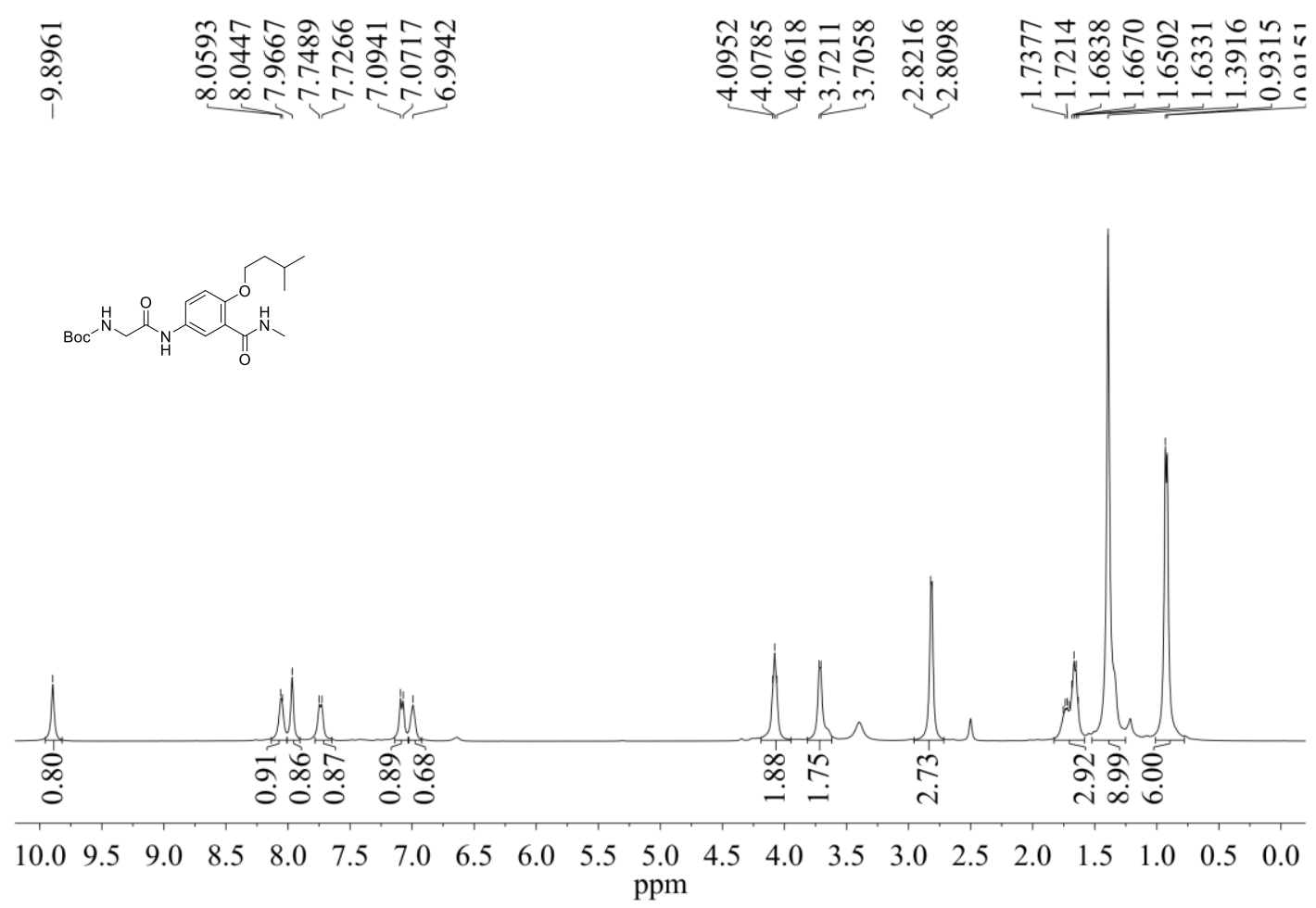

Figure S56. ${ }^{1} \mathrm{H}$ NMR spectra of compound 22 (DMSO-d $d_{6}, 298 \mathrm{~K}, 400 \mathrm{MHz}$ )
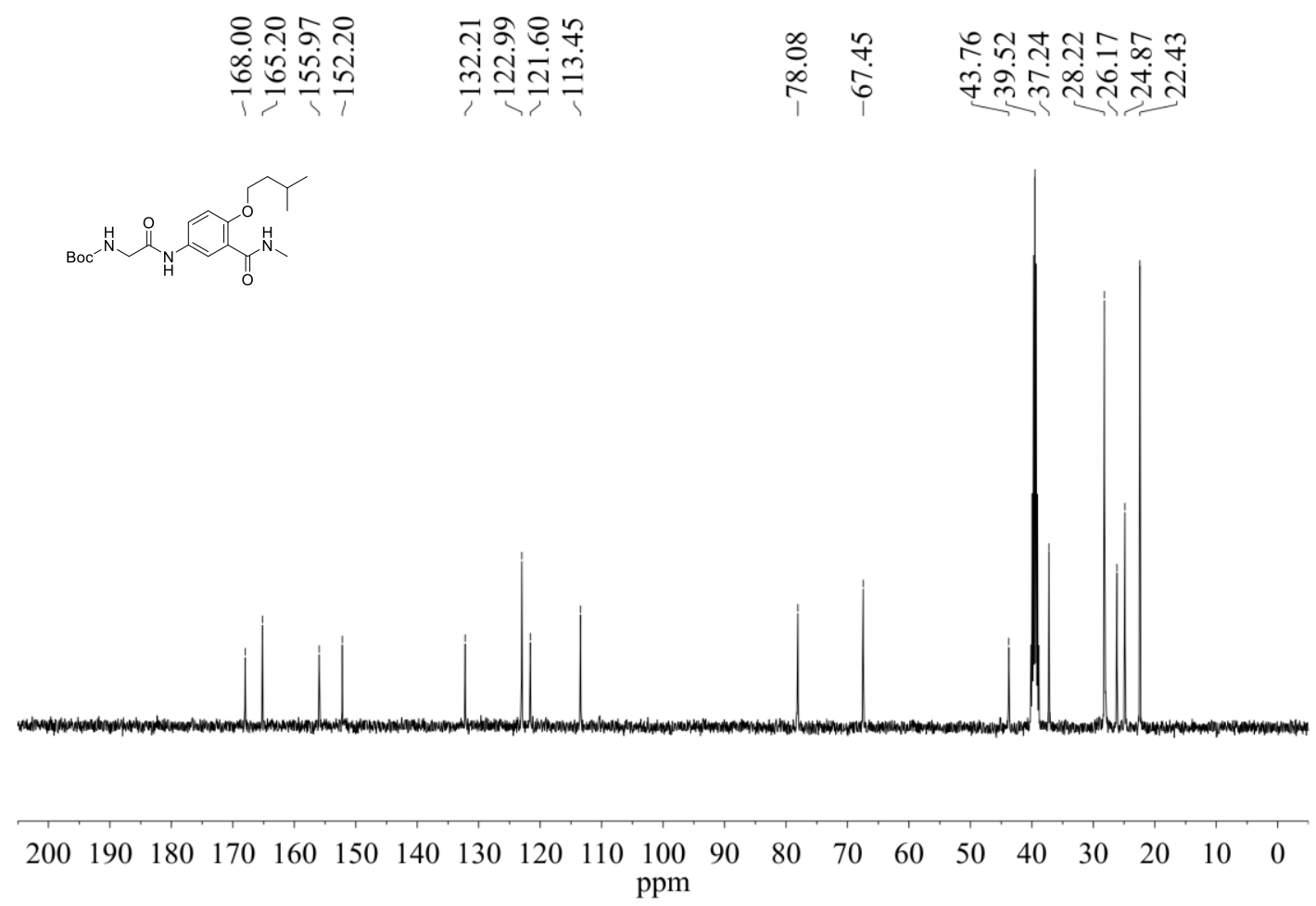

Figure S57. ${ }^{13} \mathrm{C}$ NMR spectra of compound $22\left(\mathrm{DMSO}-d_{6}, 298 \mathrm{~K}, 101 \mathrm{MHz}\right.$ ) 

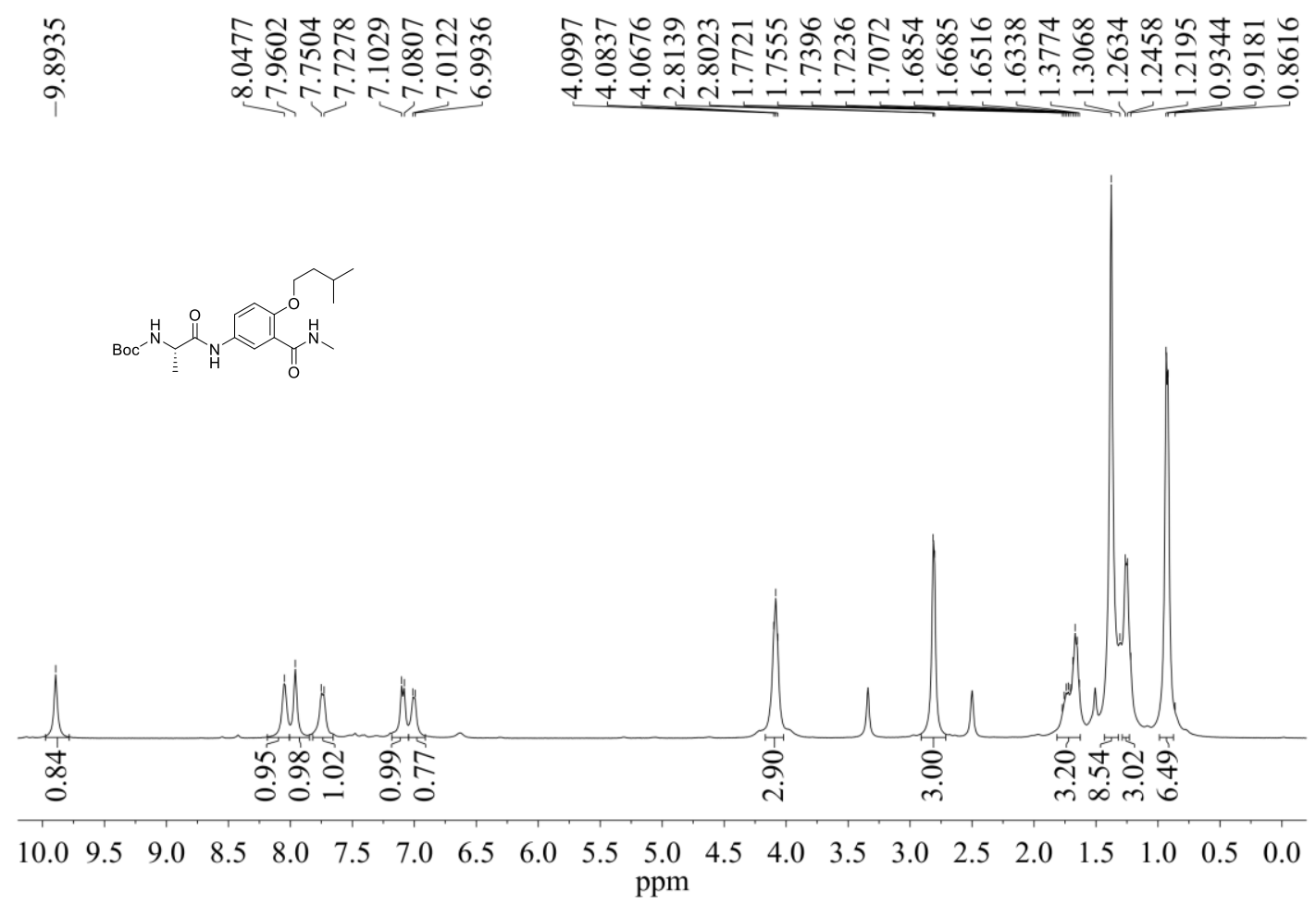

Figure S58. ${ }^{1} \mathrm{H}$ NMR spectra of compound 23 (DMSO- $d_{6}, 298 \mathrm{~K}, 400 \mathrm{MHz}$ )

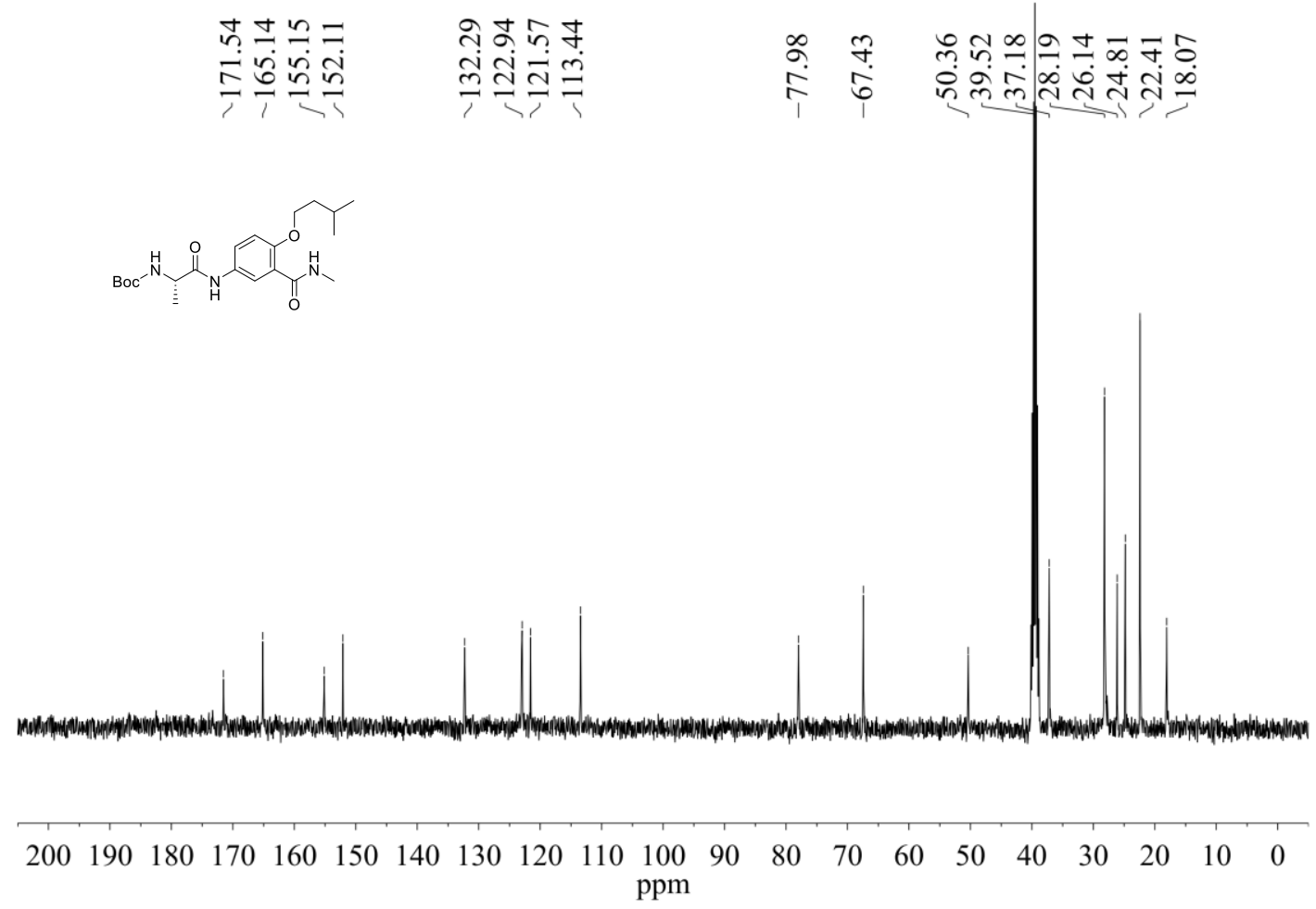

Figure S59. ${ }^{13} \mathrm{C}$ NMR spectra of compound 23 (DMSO- $d_{6}, 298 \mathrm{~K}, 101 \mathrm{MHz}$ ) 


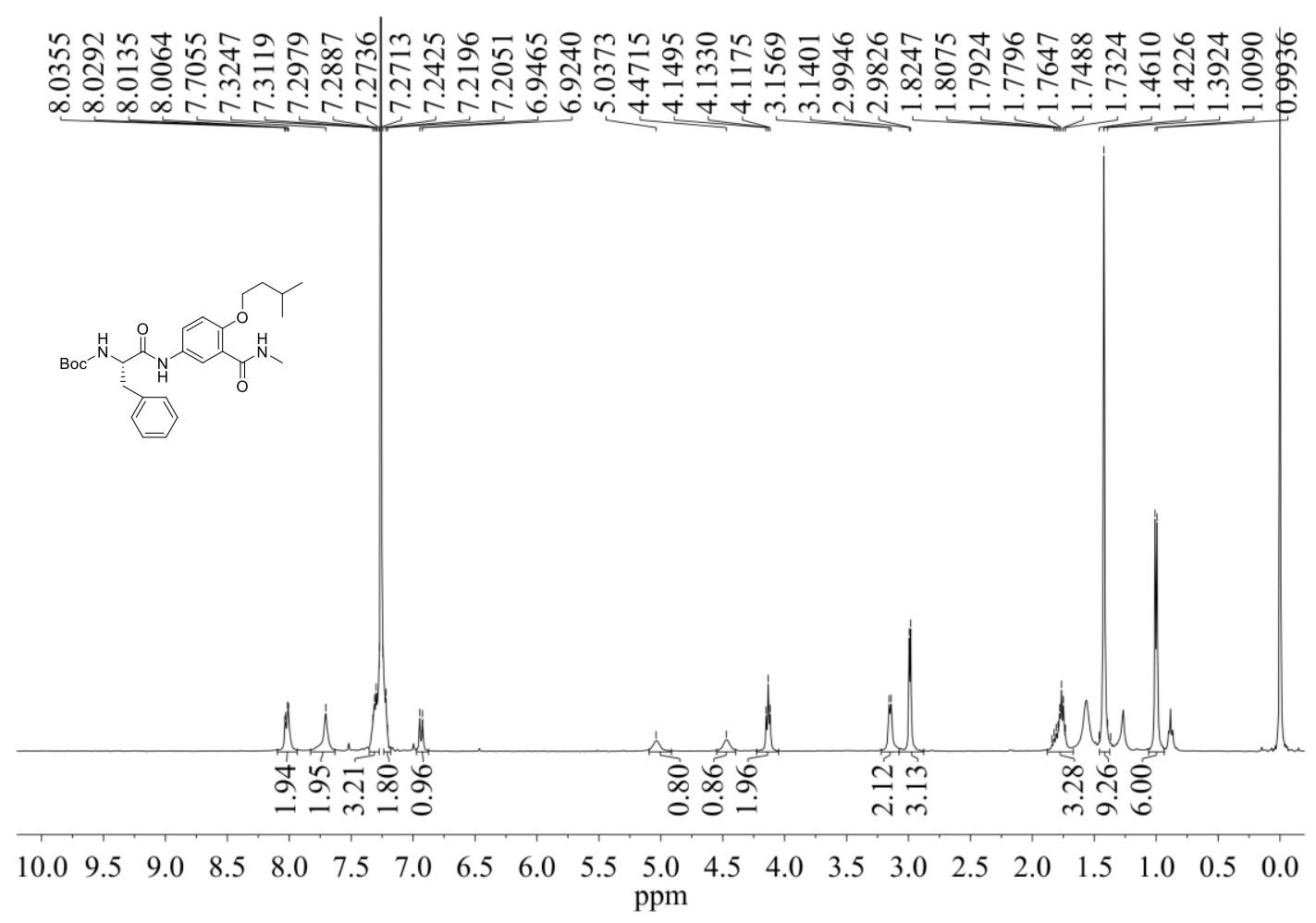

Figure S60. ${ }^{1} \mathrm{H}$ NMR spectra of compound $24\left(\mathrm{CDCl}_{3}, 298 \mathrm{~K}, 400 \mathrm{MHz}\right)$
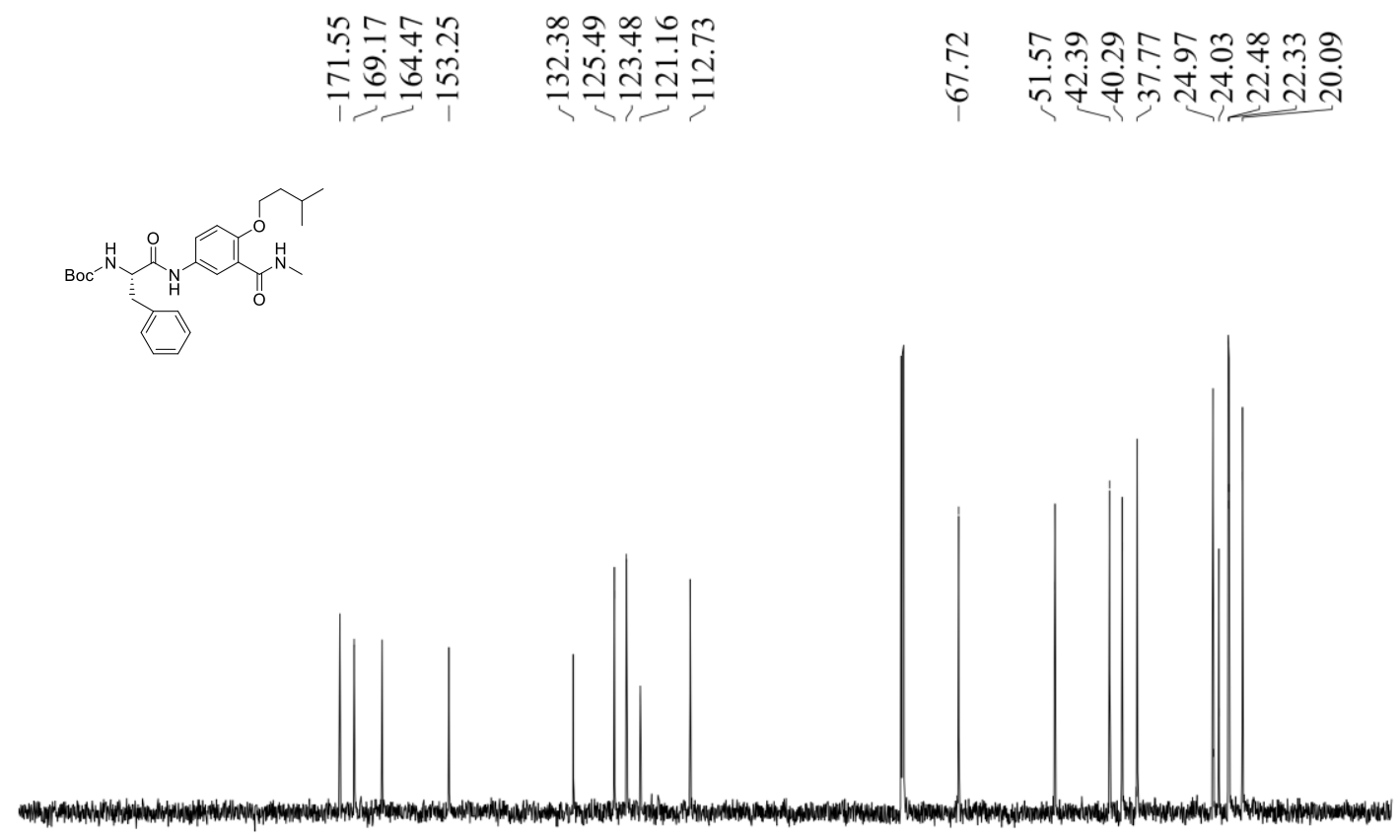

$220210200190180170160150140130120 \begin{gathered}110 \\ \mathrm{ppm}\end{gathered}$

Figure S61. ${ }^{13} \mathrm{C}$ NMR spectra of compound $24\left(\mathrm{CDCl}_{3}, 297 \mathrm{~K}, 151 \mathrm{MHz}\right)$ 


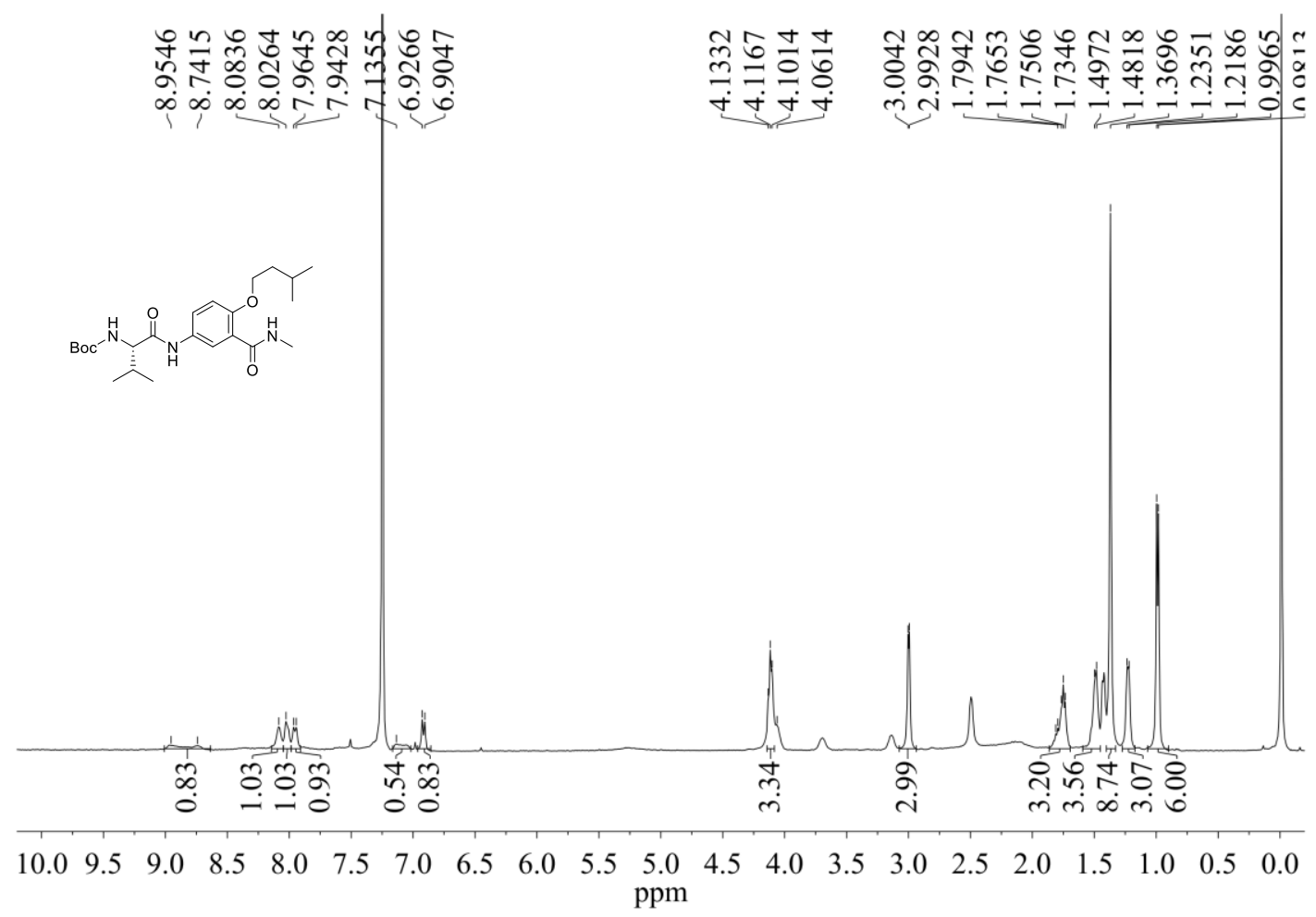

Figure S62. ${ }^{1} \mathrm{H}$ NMR spectra of compound $25\left(\mathrm{CDCl}_{3}, 302 \mathrm{~K}, 400 \mathrm{MHz}\right)$

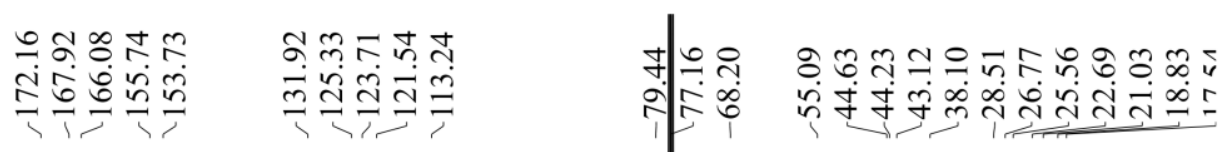

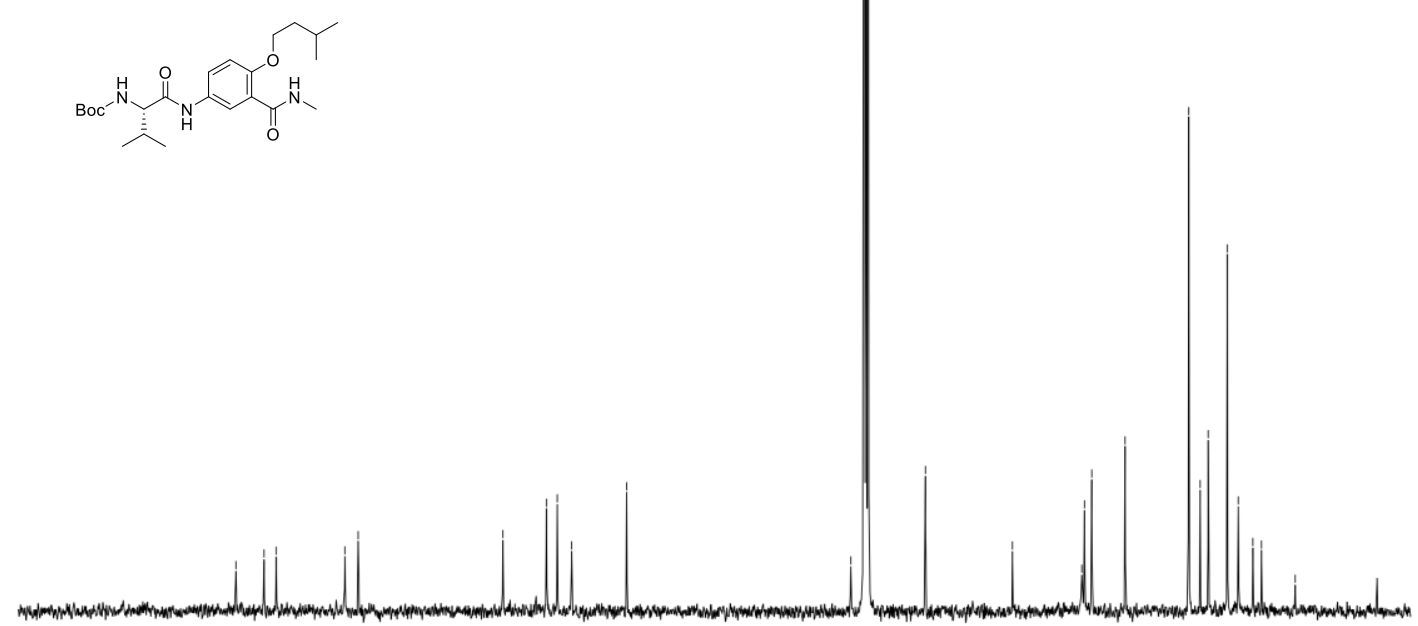

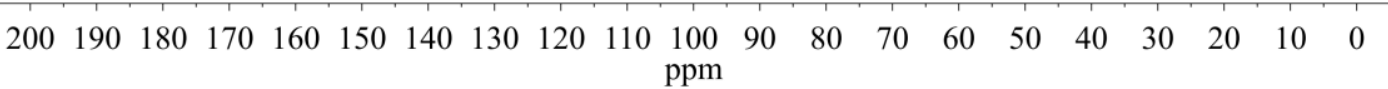

Figure S63. ${ }^{13} \mathrm{C}$ NMR spectra of compound $25\left(\mathrm{CDCl}_{3}, 308 \mathrm{~K}, 101 \mathrm{MHz}\right)$ 


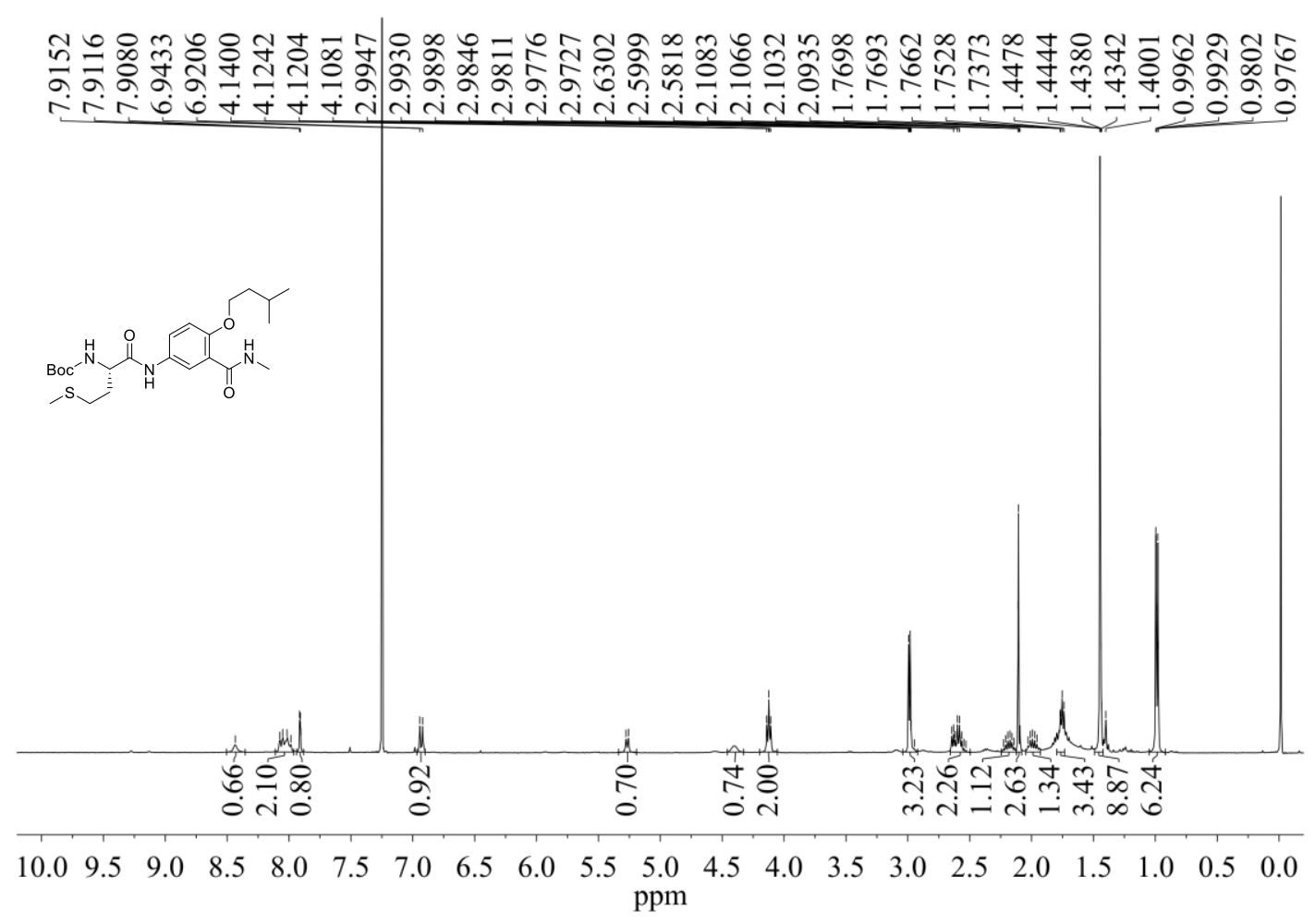

Figure S64. ${ }^{1} \mathrm{H}$ NMR spectra of compound $26\left(\mathrm{CDCl}_{3}, 298 \mathrm{~K}, 400 \mathrm{MHz}\right)$

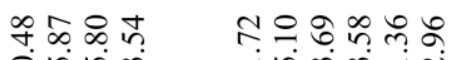

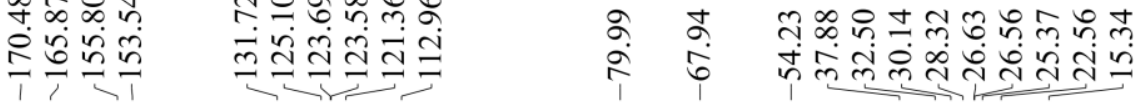

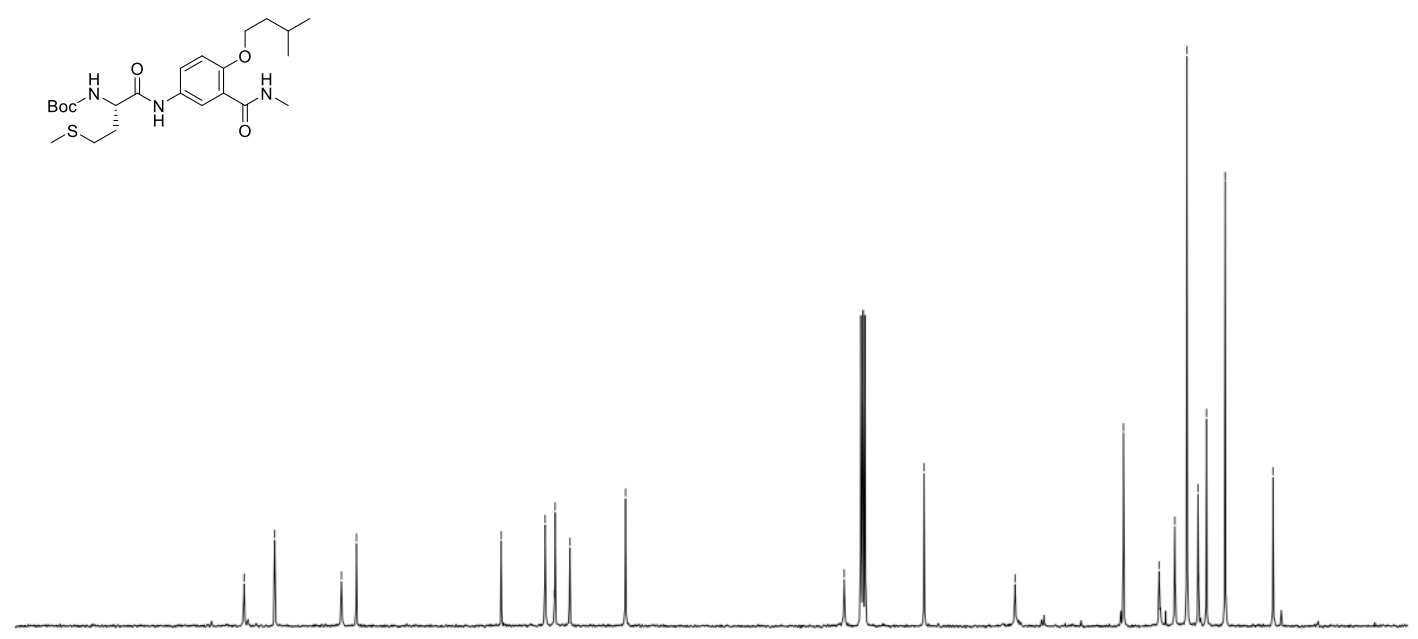

$200 \quad 190 \quad 180 \quad 170 \quad 160 \begin{array}{llllllllllllllllllll}150 & 140 & 130 & 120 & 110 & 100 & 90 & 80 & 70 & 60 & 50 & 40 & 30 & 20 & 10 & 0 \\ \mathrm{ppm}\end{array}$

Figure S65. ${ }^{13} \mathrm{C}$ NMR spectra of compound $26\left(\mathrm{CDCl}_{3}, 293 \mathrm{~K}, 101 \mathrm{MHz}\right)$ 


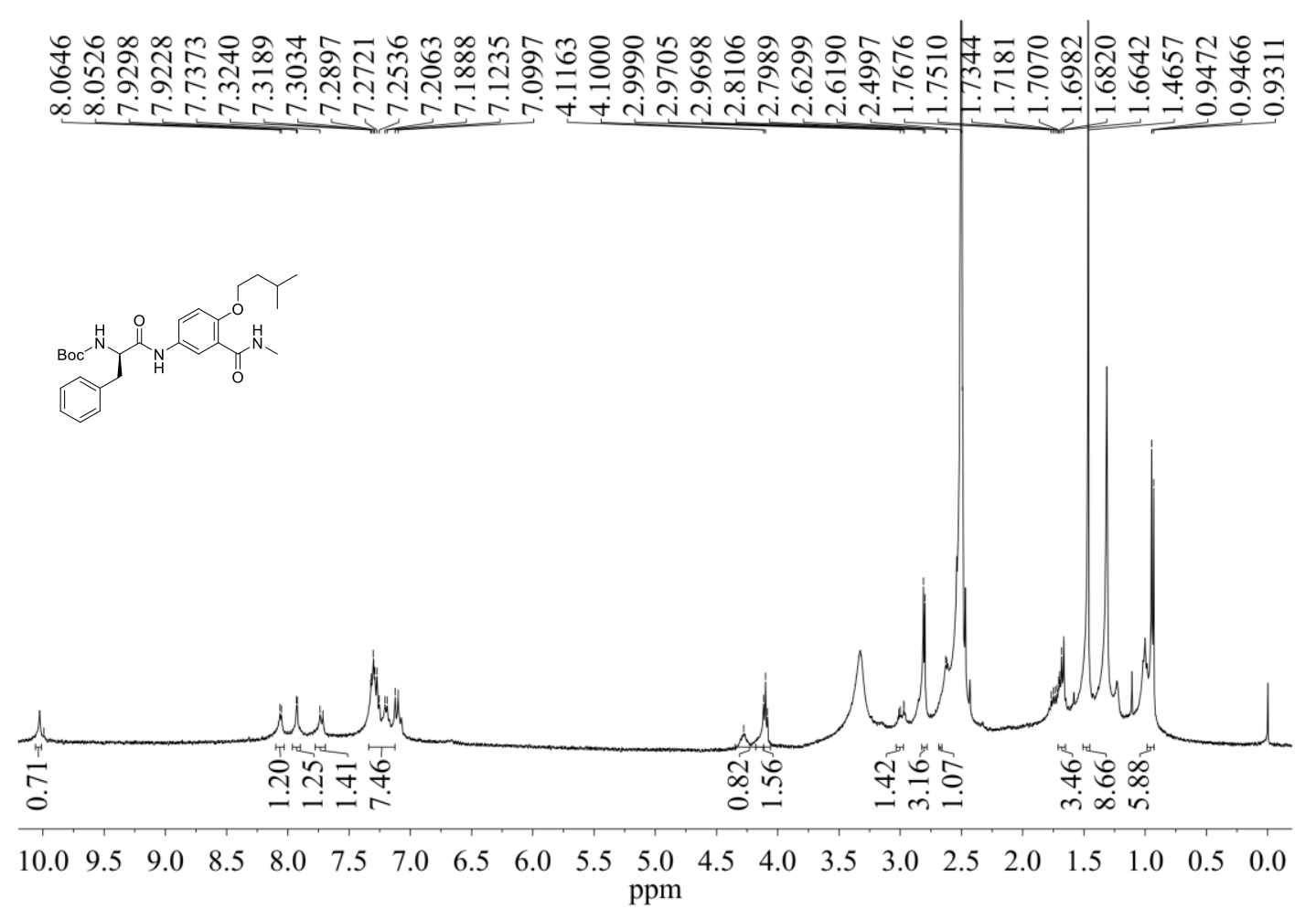

Figure S66. ${ }^{1} \mathrm{H}$ NMR spectra of compound 27 (DMSO-d $d_{6}, 296 \mathrm{~K}, 400 \mathrm{MHz}$ )
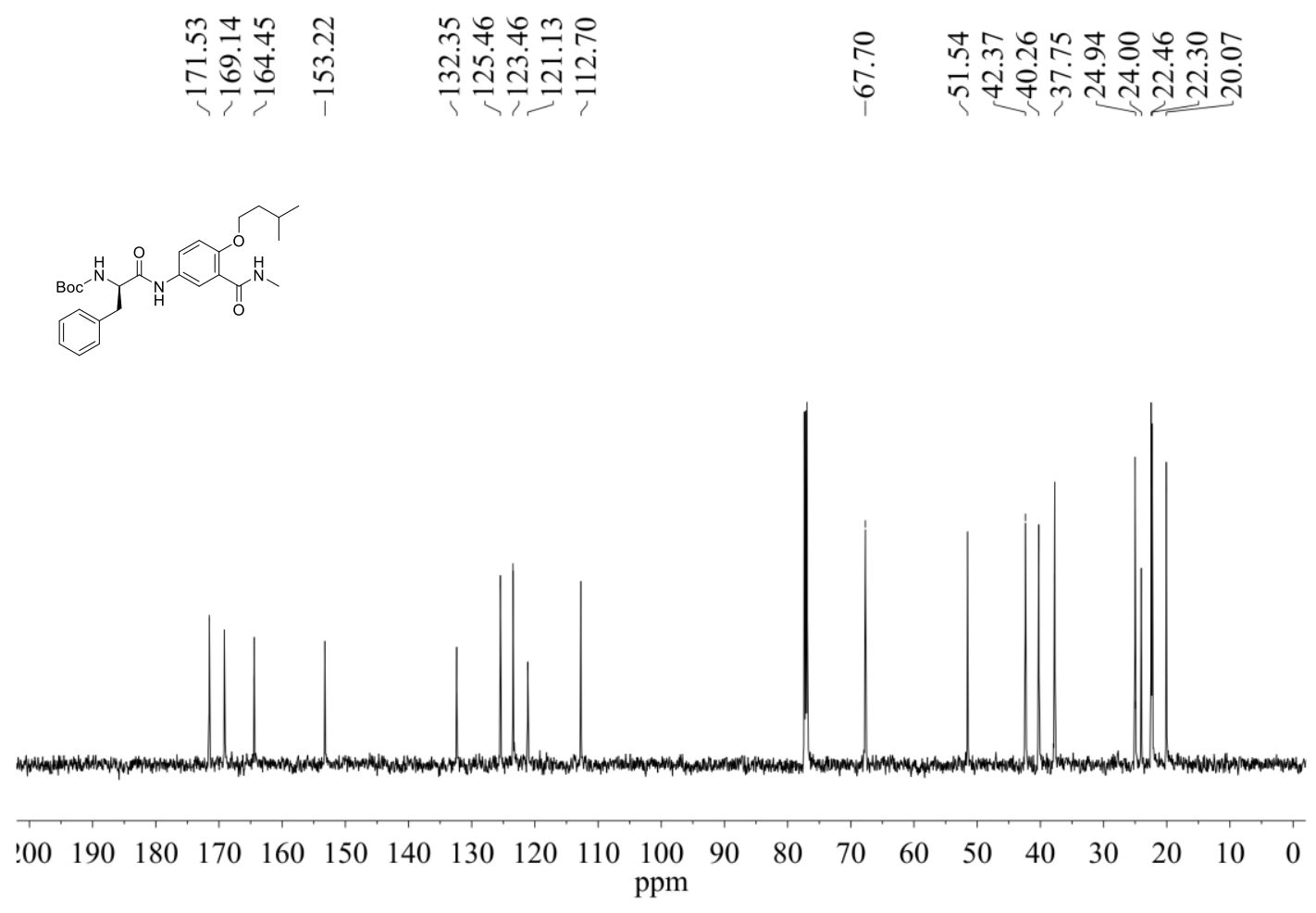

Figure S67. ${ }^{13} \mathrm{C}$ NMR spectra of compound $27\left(\mathrm{CDCl}_{3}, 297 \mathrm{~K}, 151 \mathrm{MHz}\right)$ 

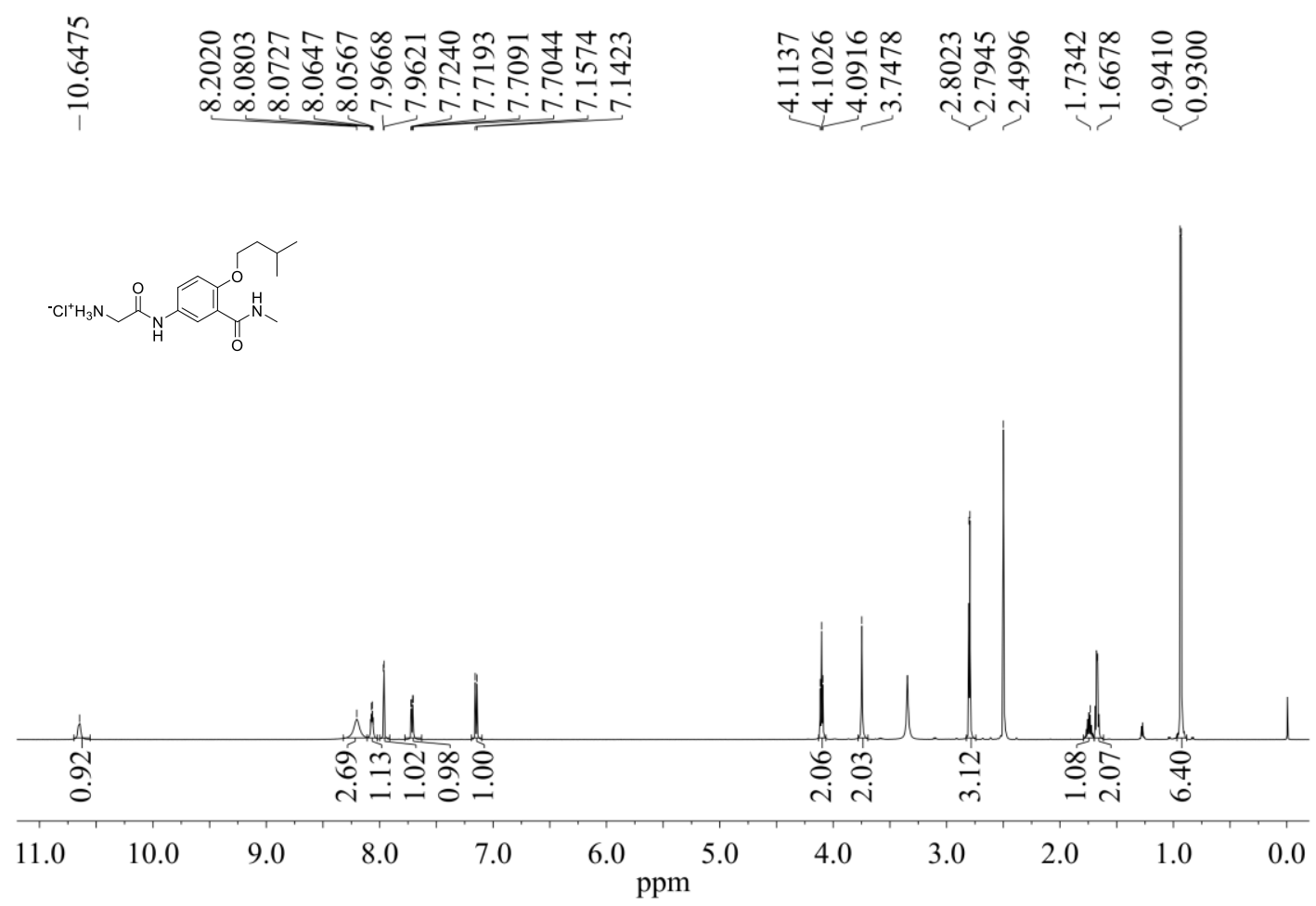

Figure S68. ${ }^{1} \mathrm{H}$ NMR spectra of compound 28 (DMSO- $\left.d_{6}, 293 \mathrm{~K}, 600 \mathrm{MHz}\right)$
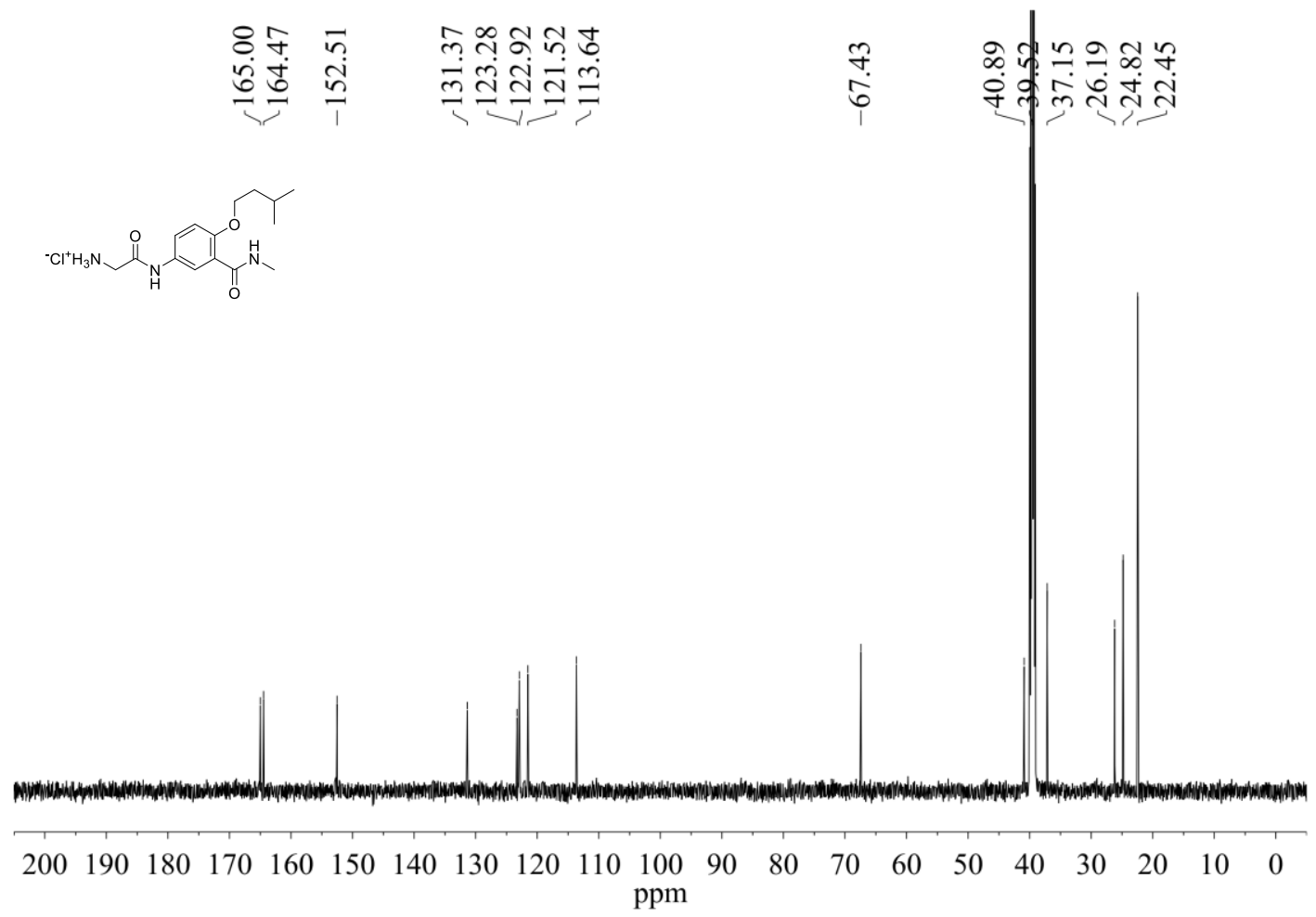

Figure S69. ${ }^{13} \mathrm{C}$ NMR spectra of compound 28 (DMSO- $d_{6}, 295 \mathrm{~K}, 151 \mathrm{MHz}$ ) 


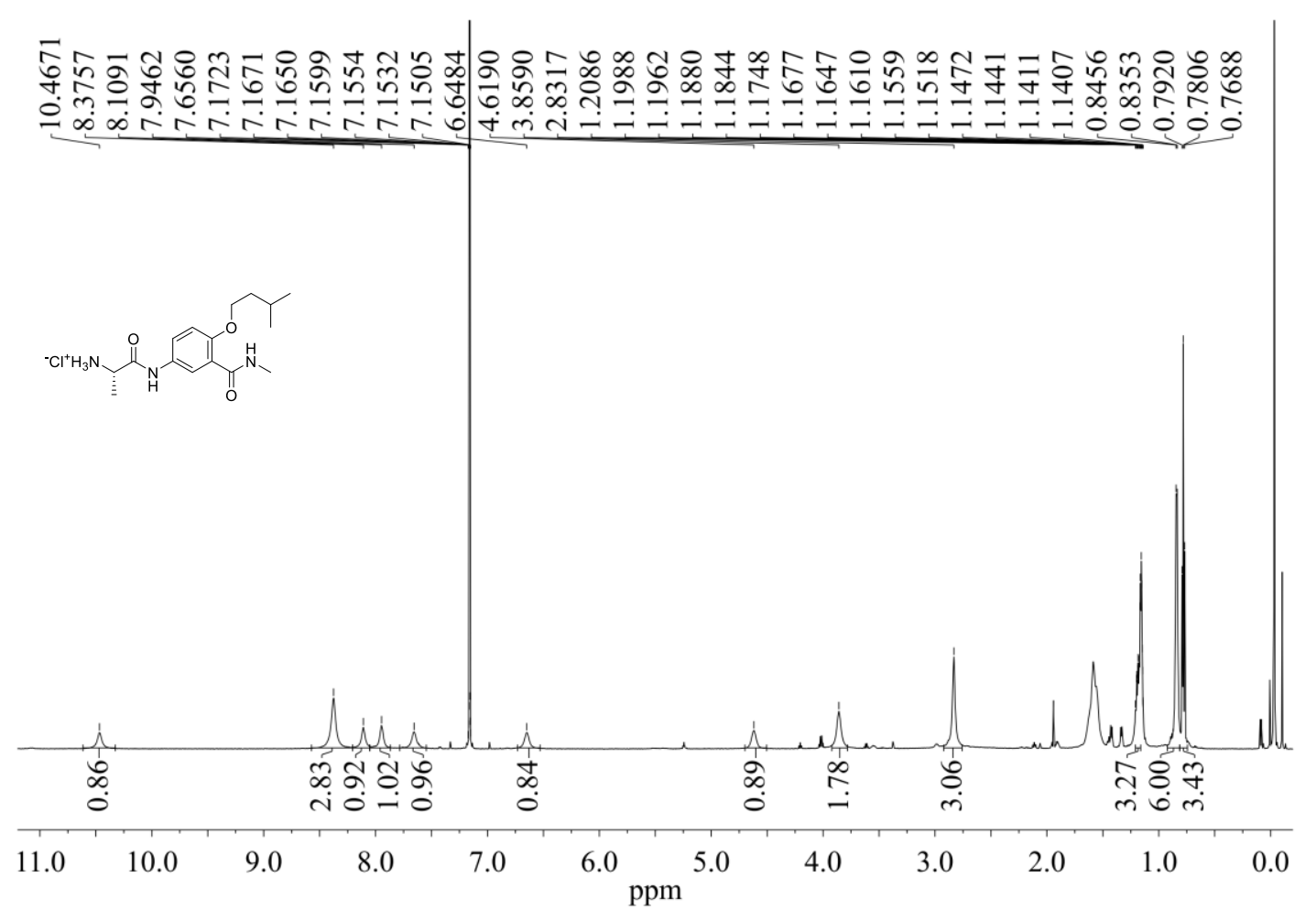

Figure S70. ${ }^{1} \mathrm{H}$ NMR spectra of compound $29\left(\mathrm{CDCl}_{3}, 298 \mathrm{~K}, 600 \mathrm{MHz}\right)$

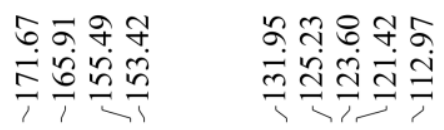

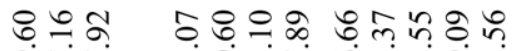

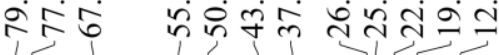
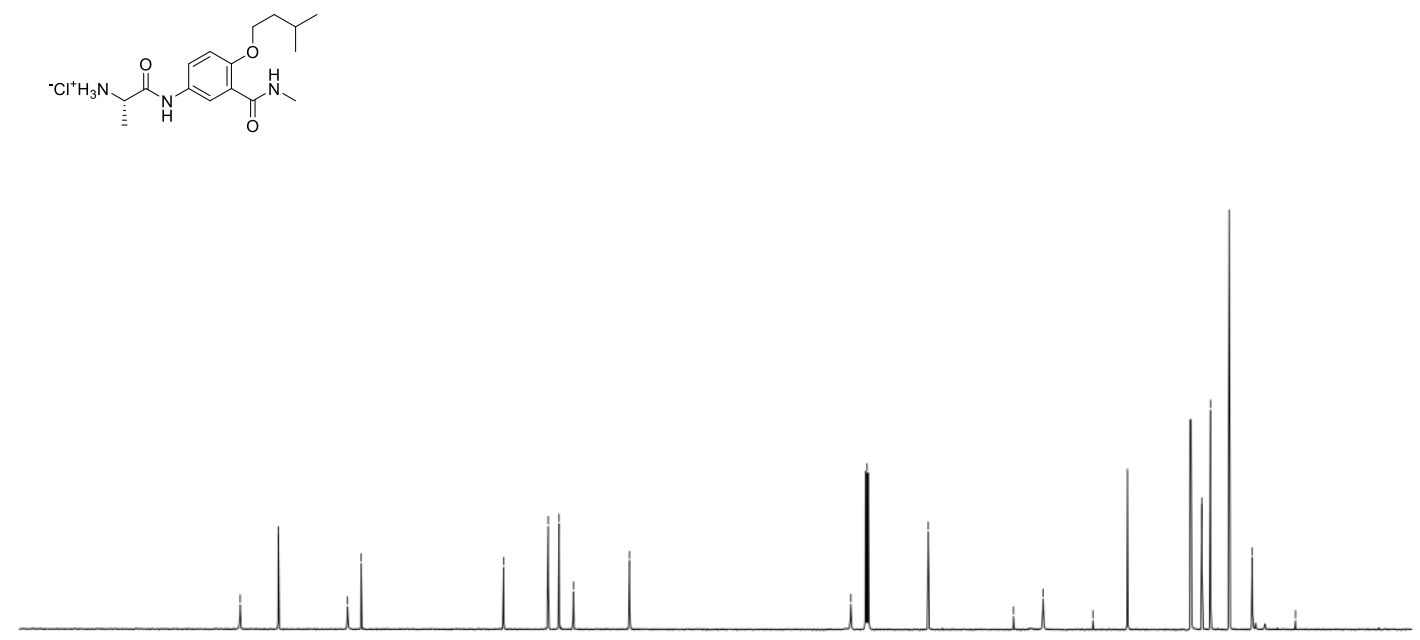

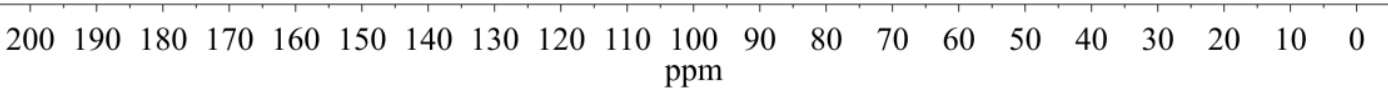

Figure S71. ${ }^{13} \mathrm{C}$ NMR spectra of compound $29\left(\mathrm{CDCl}_{3}, 296 \mathrm{~K}, 151 \mathrm{MHz}\right)$ 

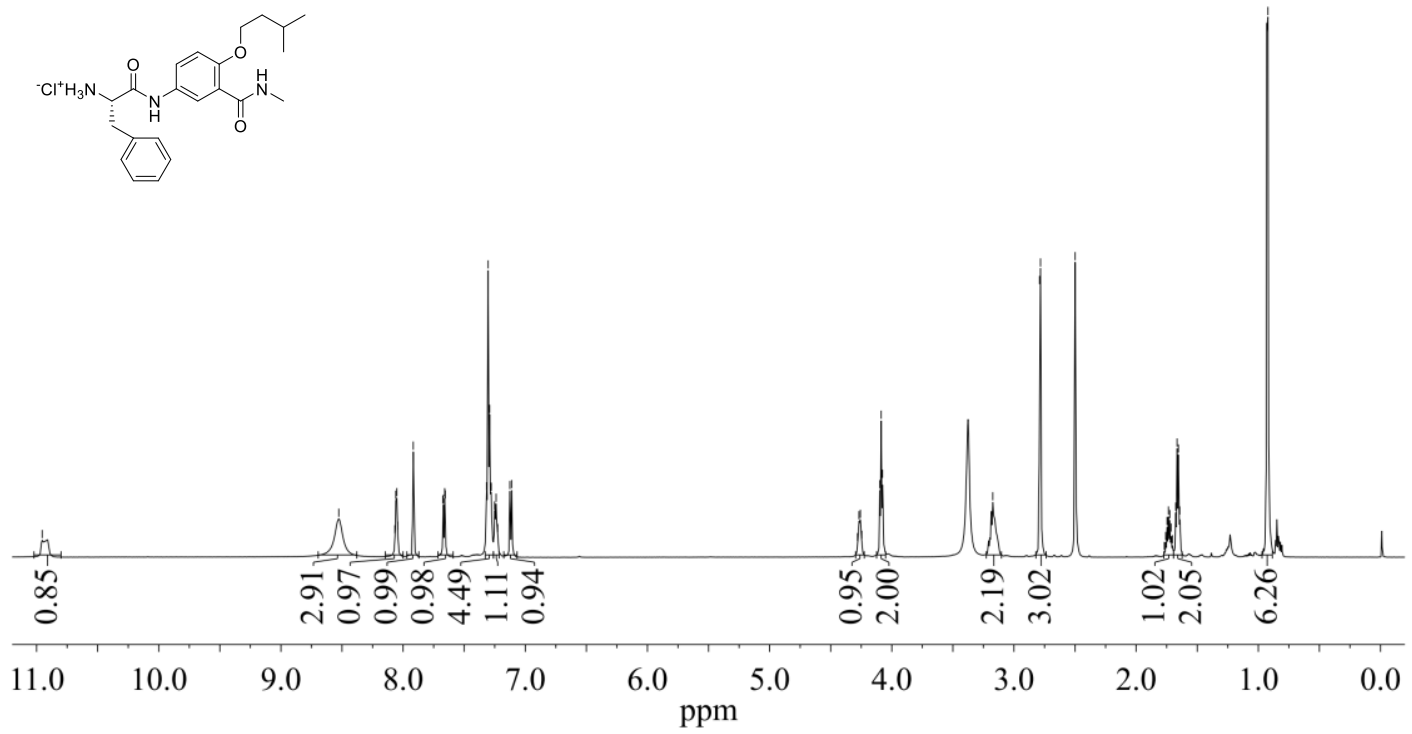

Figure S72. ${ }^{1} \mathrm{H}$ NMR spectra of compound 30 (DMSO-d $d_{6}, 293 \mathrm{~K}, 600 \mathrm{MHz}$ )
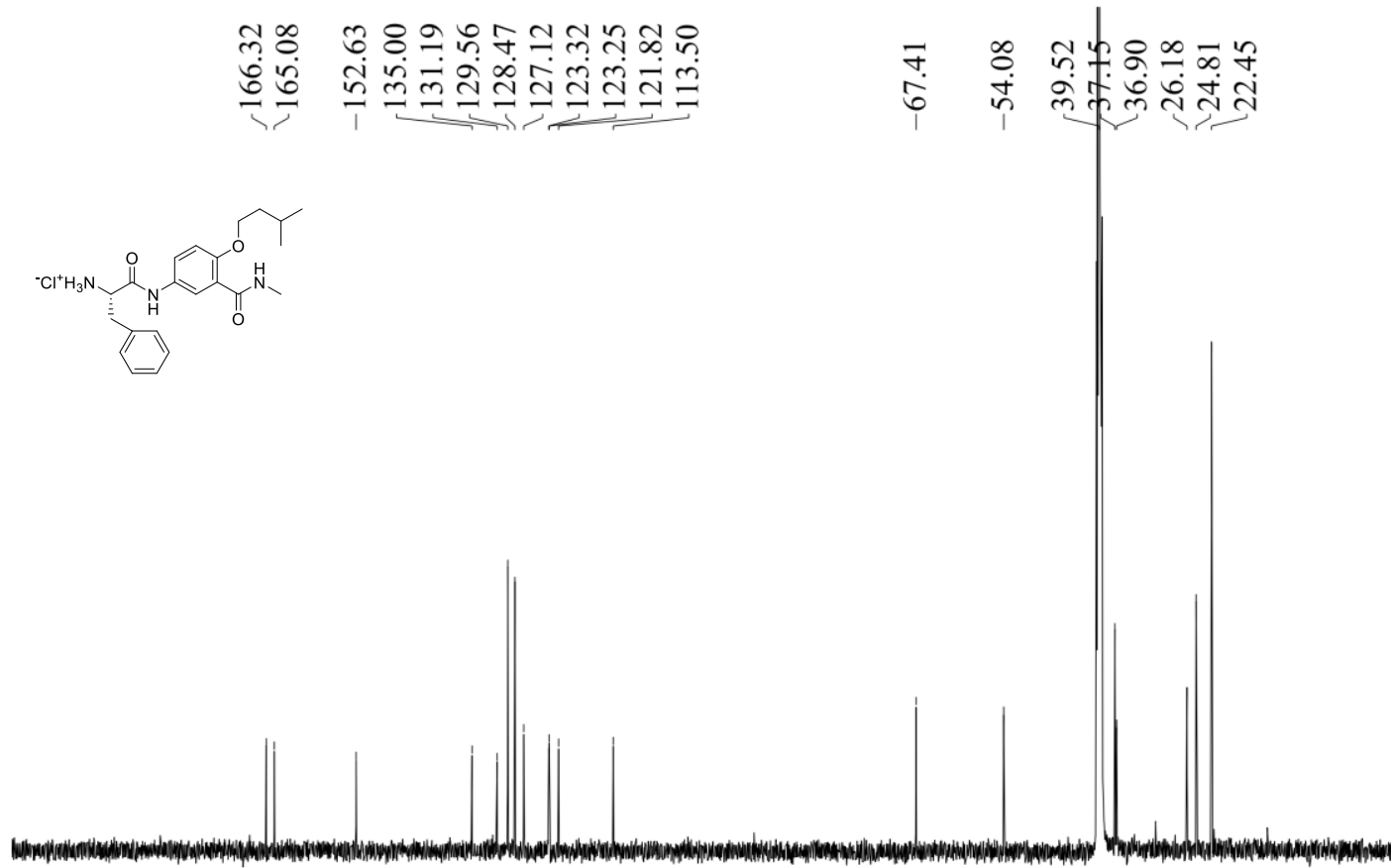

$$
\begin{array}{lllllllllllllllllllll}
200 & 190 & 180 & 170 & 160 & 150 & 140 & 130 & 120 & 110 & \begin{array}{c}
100 \\
\mathrm{ppm}
\end{array} & 90 & 80 & 70 & 60 & 50 & 40 & 30 & 20 & 10 & 0
\end{array}
$$

Figure S73. ${ }^{13} \mathrm{C}$ NMR spectra of compound 30 (DMSO- $d_{6}, 295 \mathrm{~K}, 151 \mathrm{MHz}$ ) 

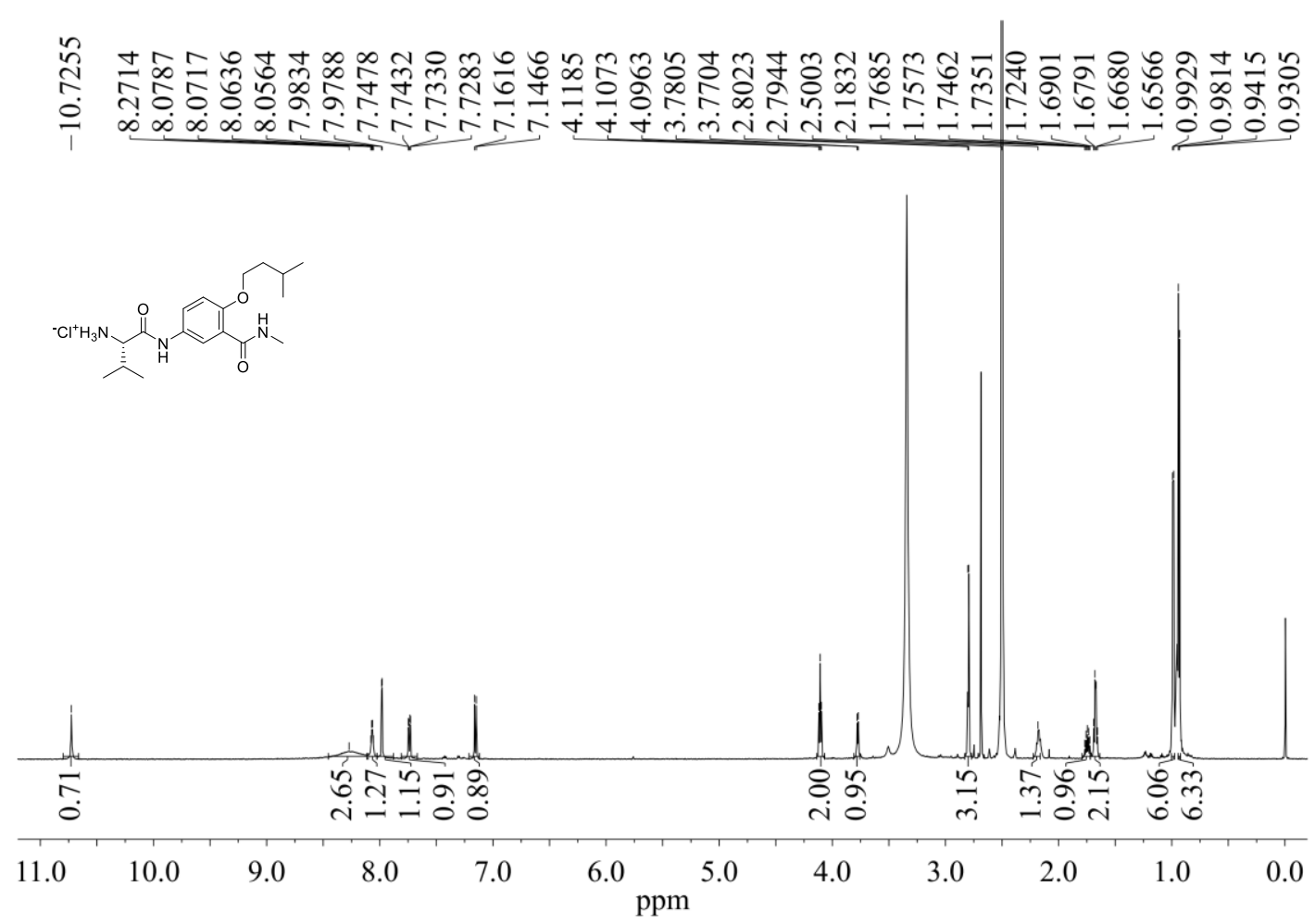

Figure S74. ${ }^{1} \mathrm{H}$ NMR spectra of compound 31 (DMSO-d $d_{6}, 294 \mathrm{~K}, 600 \mathrm{MHz}$ )
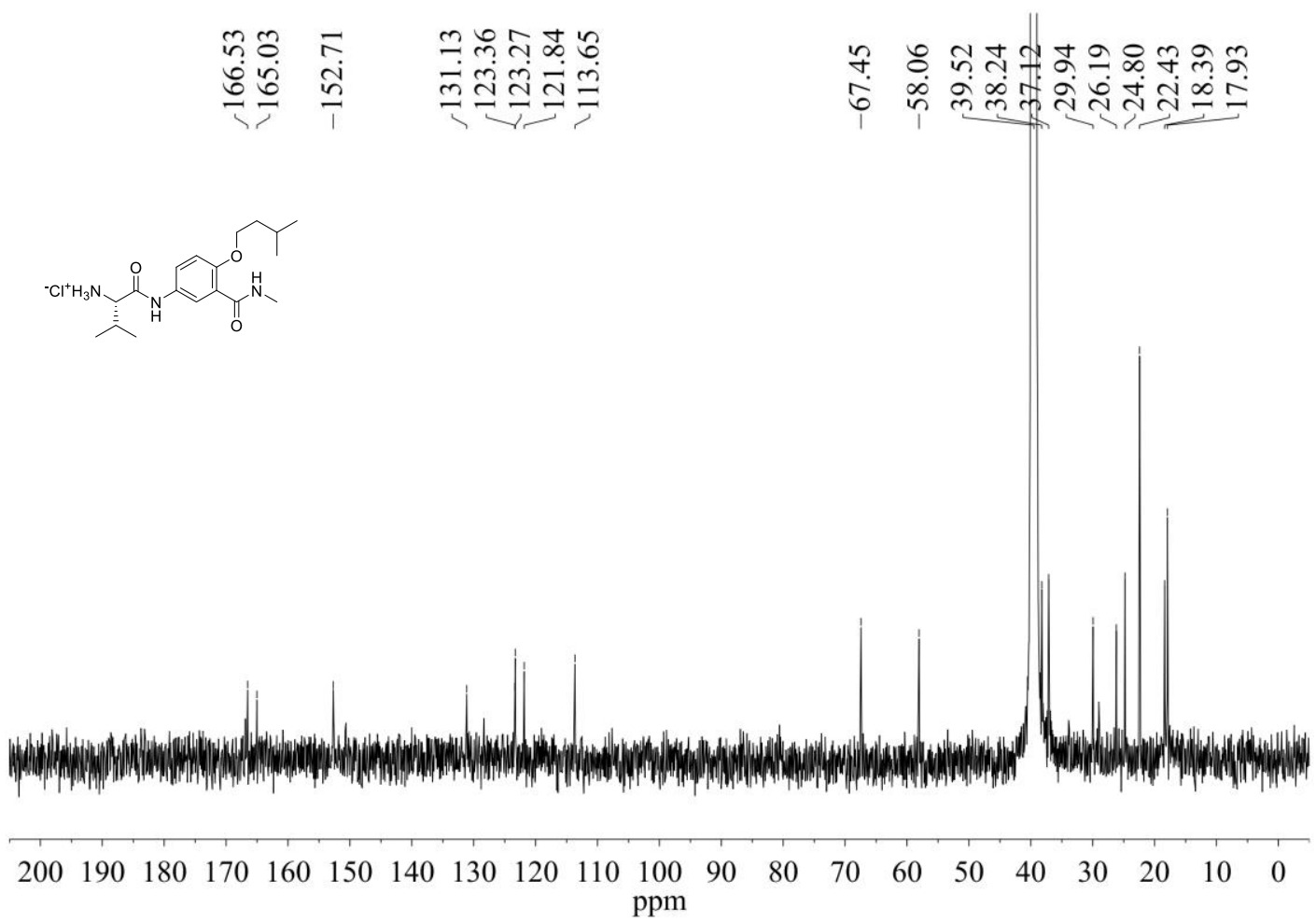

Figure S75. ${ }^{13} \mathrm{C}$ NMR spectra of compound 31 (DMSO- $d_{6}, 296 \mathrm{~K}, 151 \mathrm{MHz}$ ) 


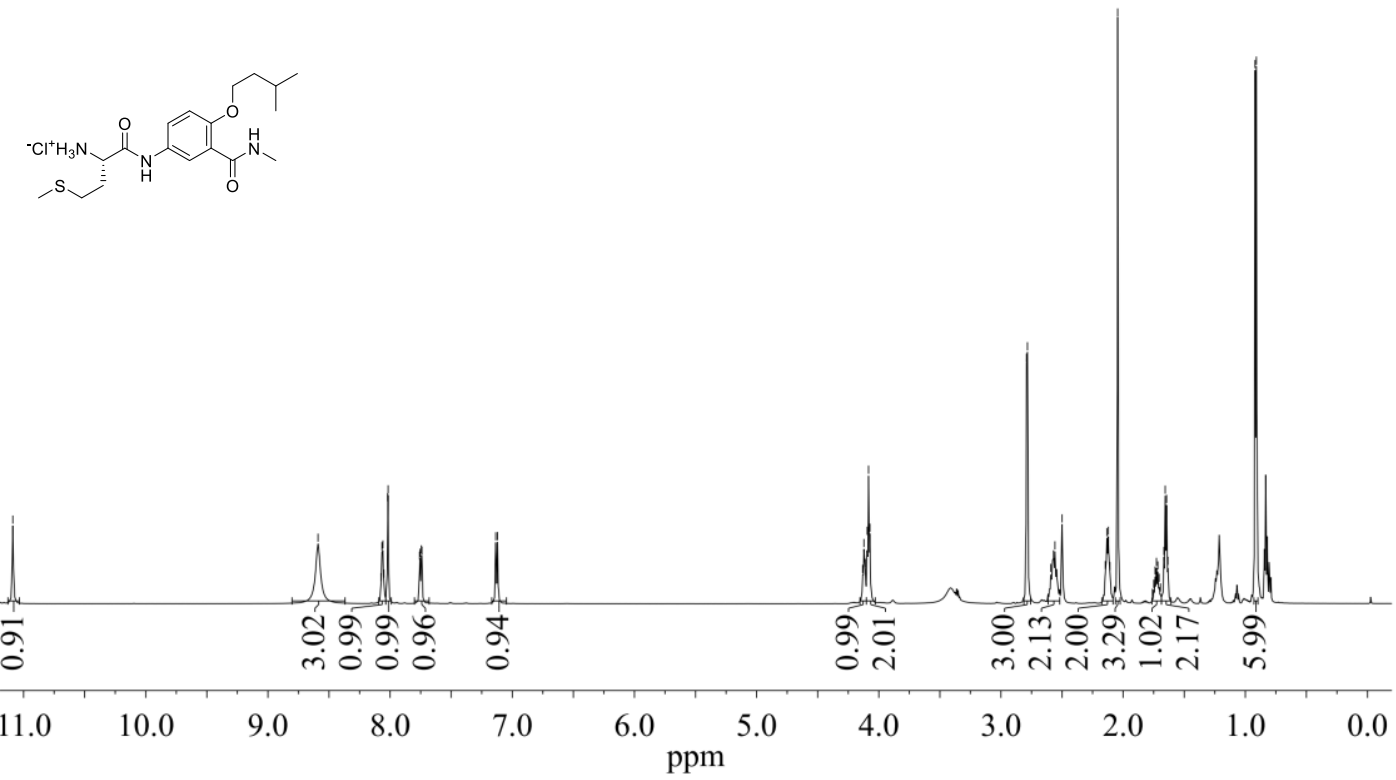

Figure S76. ${ }^{1} \mathrm{H}$ NMR spectra of compound 32 (DMSO-d $d_{6}, 293 \mathrm{~K}, 600 \mathrm{MHz}$ )
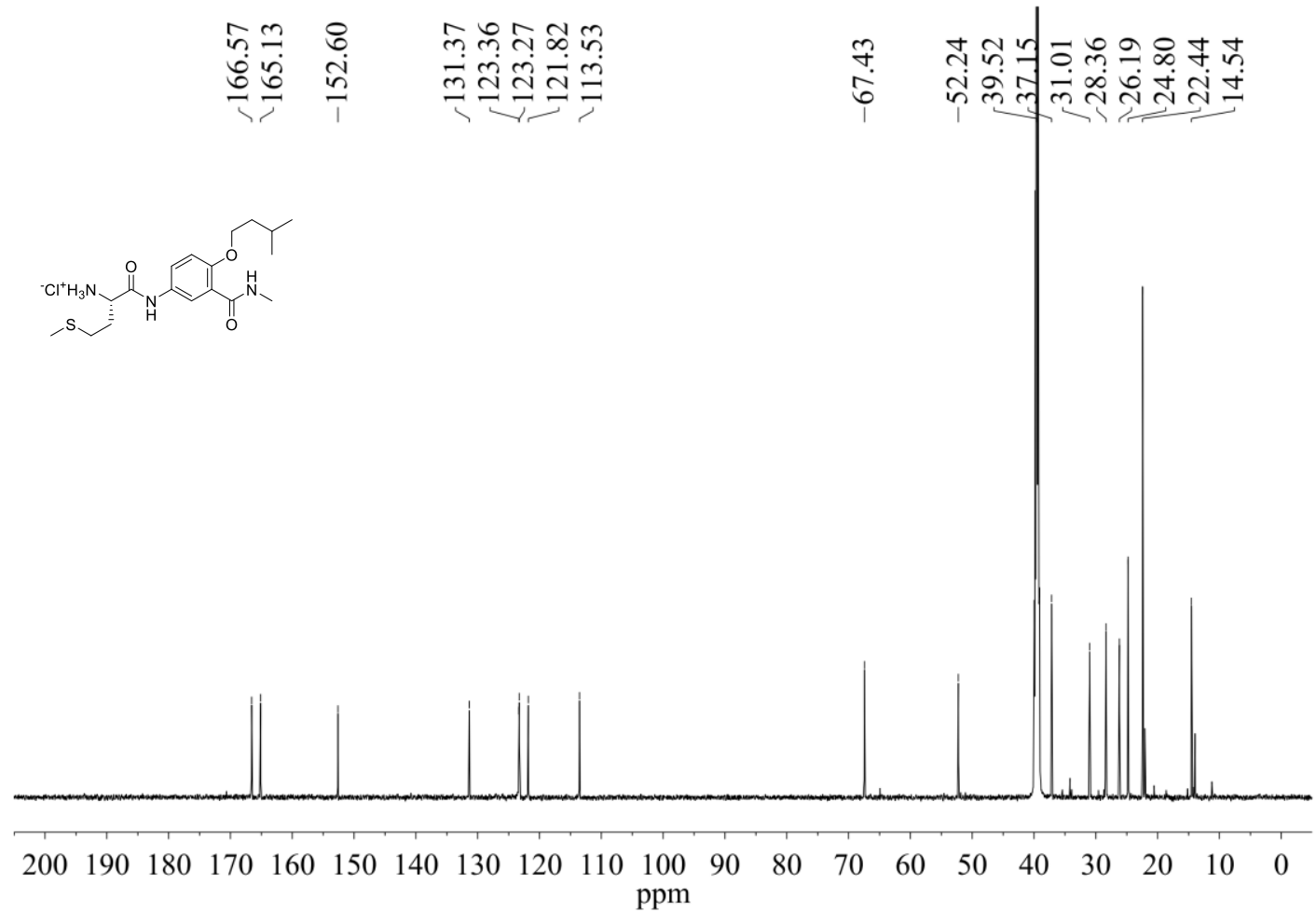

Figure S77. ${ }^{13} \mathrm{C}$ NMR spectra of compound 32 (DMSO- $d_{6}, 295 \mathrm{~K}, 151 \mathrm{MHz}$ ) 

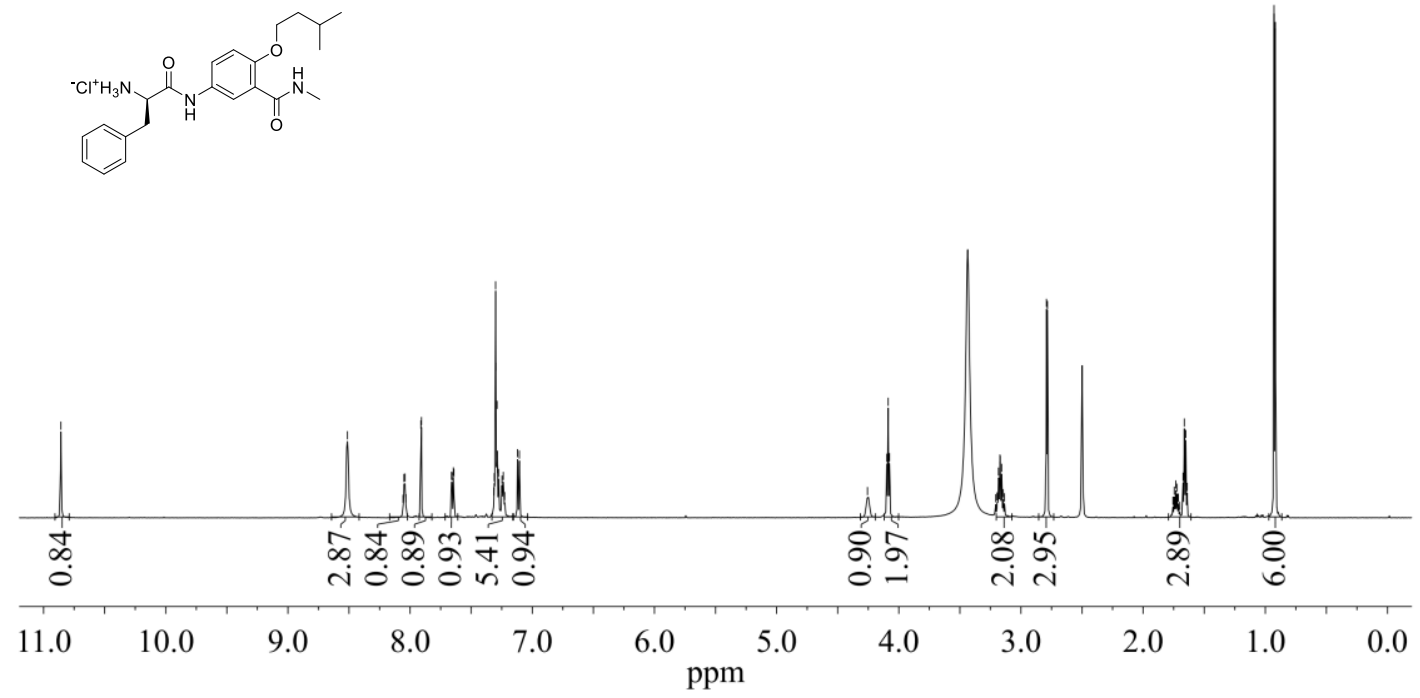

Figure S78. ${ }^{1} \mathrm{H}$ NMR spectra of compound 33 (DMSO-d $d_{6}, 298 \mathrm{~K}, 600 \mathrm{MHz}$ )
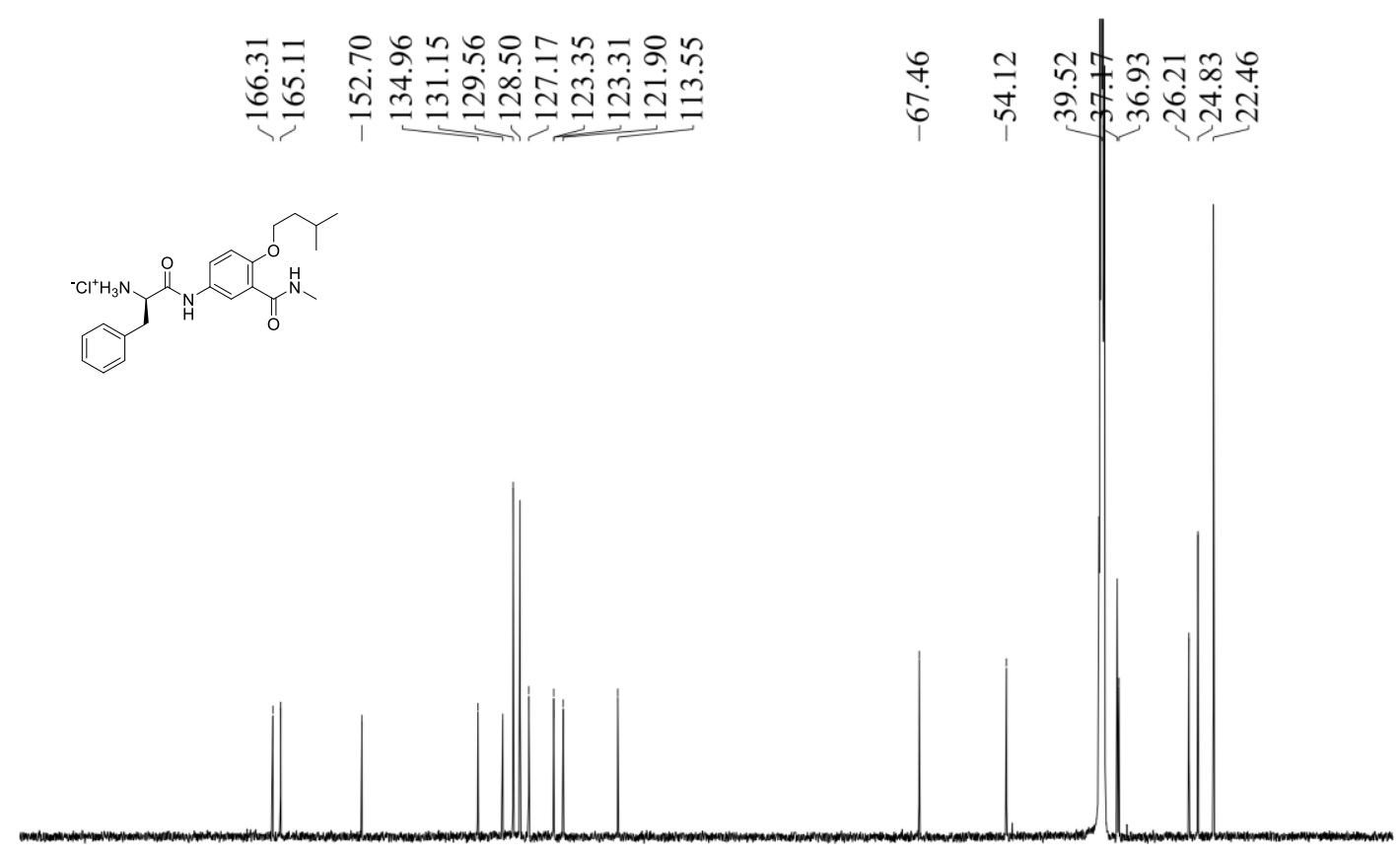

$\begin{array}{lllllllllllllllllllll}200 & 190 & 180 & 170 & 160 & 150 & 140 & 130 & 120 & 110 & \begin{array}{c}100 \\ \mathrm{ppm}\end{array} & 90 & 80 & 70 & 60 & 50 & 40 & 30 & 20 & 10 & 0\end{array}$

Figure S79. ${ }^{13} \mathrm{C}$ NMR spectra of compound 33 (DMSO- $\left.d_{6}, 299 \mathrm{~K}, 151 \mathrm{MHz}\right)$ 


\section{Computational Study}

The computations on the $\mathbf{2} \mathbf{i}$ and $\mathbf{2} \mathbf{j}$ models were performed with revPBE-D $3^{4,5}$ using the $\mathrm{ADF}^{6-8}$ software package. The triple-zeta with polarization functions (TZP) basis set was used within each calculation. ${ }^{9}$ The core $1 \mathrm{~s}$ electrons for oxygen, nitrogen, and carbon were kept fixed in the DFT-D3 calculations. 


\section{References}

1. Incerti, M.; Russo, S.; Callegari, D.; Pala, D.; Giorgio, C.; Zanotti, I.; Barocelli, E.; Vicini, P.; Vacondio, F.; Rivara, S.; Castelli, R.; Tognolini, M.; Lodola, A., J. Med. Chem. 2017, 60, 787.

2. Yang, S.; Wei, Y. WO2017198122A1, 2017.

3. Zhang, Y.; Cao, R.; Shen, J.; Detchou, C. S. F.; Zhong, Y.; Wang, H.; Zou, S.; Huang, Q.; Lian, C.; Wang, Q.; Zhu, J.; Gong, B., Org. Lett. 2018, 20, 1555.

4. Y. Zhang \& W. Yang, Phys. Rev. Lett. 1998, 80, 890.

5. S. Grimme, J. Antony, S. Ehrlich \& H. Krieg, J. Chem. Phys. 2010, 132, 154104.

6. G. te Velde, F. M. Bickelhaupt, S. J. A. van Gisbergen, C. Fonseca Guerra, E. J. Baerends, J. G. Snijders \& T. Ziegler, J. Comp. Chem. 2001, 22, 931.

7. C. Fonseca Guerra, J. G. Snijders, G. te Velde \& E. J. Baerends, Theor. Chem. Acc. 1998, 99, 391.

8. ADF, SCM, Theoretical Chemistry, Vrije Universiteit, Amsterdam, The Netherlands, http://www.scm.com.

9. E. van Lenthe \& E. J. Baerends, J. Comp. Chem. 2003, 24, 1142. 